\title{
Laboratory Directed Research and Development
}

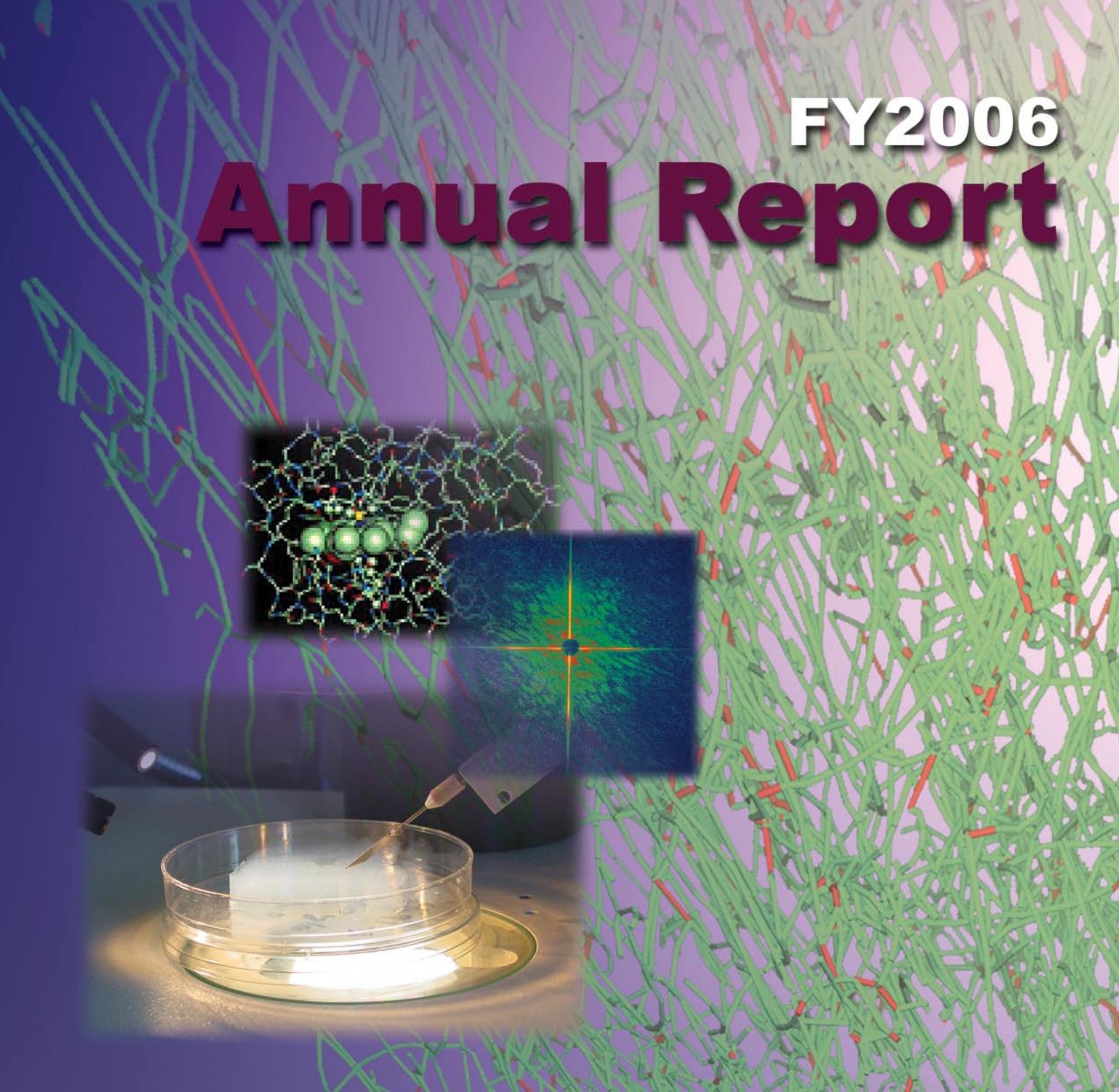




\section{About the Cover:}

The LDRD project 06-LW-028, "Diffusion Monte Carlo without All the Hops" (background and top image) is developing a new method for diffusion Monte Carlo simulations that will allow researchers to perform simulations more quickly and utilize supercomputer resources more efficiently. Ultimately, this will open the door to new opportunities for predictive modeling of complex processes that are relevant to stockpile science and technology and to energy science and security, but for which standard diffusion Monte Carlo is ineffective.

Single-molecule atomic-resolution imaging-which will allow the structure of virtually any macromolecule, protein, or virus to be determined-is the goal of LDRD project 05-SI-003, "Biological Imaging with Fourth-Generation Light Sources" (center). Diffraction patterns recorded with a single 25 -femtosecond $x$-ray pulse from a soft-x-ray free-electron laser allows researchers to reconstruct an image of the object that gave rise to the pattern. The technique can be applied all the way to atomic resolution once hard-x-ray free-electron lasers become available.

Cometary particles collected by the Stardust mission and extracted from the silica aerogel grids onboard the spacecraft were analyzed using transmission electron microscopy, focused ion beam-field emission scanning electron microscopy, and synchrotron beamlines by Livermore researchers and others. Materials that have never been remotely detected in a comet were discovered, indicating that much more mixing occurred in the early solar system than was previously thought. The research was part of LDRD project 06-ERI-001, "Development of Integrated Microanalysis of Nanomaterials" (bottom).

\section{UCRL-TR-113717-06}

This report has been reproduced directly from the best available copy. Available to DOE and DOE contractors from the Office of Scientific and Technical Information

P.O. Box 62, Oak Ridge, TN 37831

Prices available from (423) 576-8401

http://apollo.osti.gov/bridge/

Available to the public from the National Technical Information Service U.S. Department of Commerce 5285 Port Royal Rd., Springfield, VA 22161 http://www.ntis.gov/ or Lawrence Livermore National Laboratory Technical Information Department Digital Library http://www.IInl.gov/tid/Library.html

\section{Disclaimer}

This document was prepared as an account of work sponsored by an agency of the United States Government. Neither the United States Government nor the University of California nor any of their employees, makes any warranty, express or implied, or assumes any legal liability or responsibility for the accuracy, completeness, or usefulness of any information, apparatus, product, or process disclosed, or represents that its use would not infringe privately owned rights. Reference herein to any specific commercial products, process, or service by trade name, trademark, manufacturer, or otherwise, does not necessarily constitute or imply its endorsement, recommendation, or favoring by the United States Government or the University of California. The views and opinions of authors expressed herein do not necessarily state or reflect those of the United States Government or the University of California, and shall not be used for advertising or product endorsement purposes.

This work was performed under the auspices of the U.S. Department of Energy by University of California Lawrence Livermore National Laboratory under Contract No. W-7405-Eng-48. 


\section{Acknowledgments}

This Annual Report provides an overview of the FY2006 Laboratory Directed Research and Development (LDRD) Program at Lawrence Livermore National Laboratory (LLNL) and presents a summary of the results achieved by each LDRD project. At LLNL, Laboratory Director George Miller and Deputy Director for Science and Technology Cherry Murray are responsible for the LDRD Program and delegate responsibility for the operation of the program to the Associate Deputy Director for Science and Technology and the Director of the Laboratory Science and Technology Office (LSTO), Rokaya Al-Ayat. The LDRD Program at LLNL is in compliance with Department of Energy (DOE) Order 413.2 and other relevant DOE orders and guidelines.

The LDRD Program extends its sincere appreciation to the principal investigators of the FY2006 projects for providing the content of the Annual Report and to the publications team. The LDRD Program also thanks the following members of the LSTO team for their many contributions to this publication: Dolores Lambert, administrator; Nancy Campos, database manager; Steve McNamara, computer specialist; and Cathleen Sayre, resource manager.

\section{Scientific Editors}

James De Yoreo, Kenneth Jackson, Karl van Bibber

\section{Publication Editors}

Paul Kotta, Jeffrey Sketchley

\section{Publication Designer}

Denise Kellom 


\section{Director's Statement}

The Laboratory Directed Research and Development (LDRD) Program, authorized by Congress in 1991 and administered by the Laboratory Science and Technology Office, is our primary means for pursuing innovative, long-term,

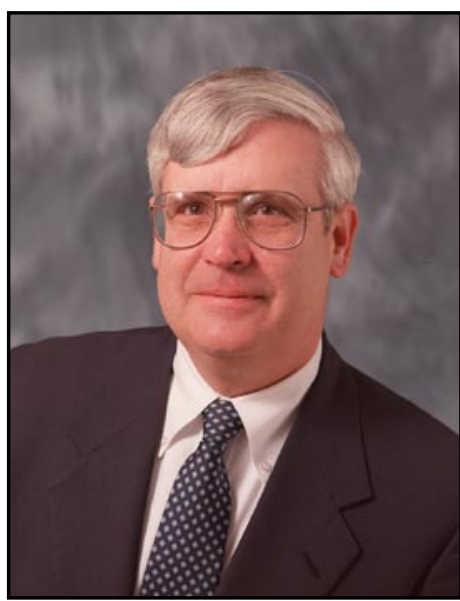

George Miller, Director high-risk, and potentially high-payoff research that supports the missions of the Laboratory, the Department of Energy, and National Nuclear Security Administration in national security, energy security, environmental management, bioscience and technology to improve human health, and breakthroughs in fundamental science and technology. The accomplishments described in this Annual Report demonstrate the strong alignment of the LDRD portfolio with these missions and contribute to the Laboratory's success in meeting its goals.

The LDRD budget of $\$ 92$ million for FY2006 sponsored 188 projects. These projects were selected through an extensive peer-review process to ensure the highest scientific quality and mission relevance. Each year, the number of deserving proposals far exceeds the funding available, making the selection a tough one indeed.

Our ongoing investments in LDRD have reaped long-term rewards for the Laboratory and the nation. Many Laboratory programs trace their roots to research thrusts that began several years ago under LDRD sponsorship. In addition, many LDRD projects contribute to more than one mission area, leveraging the Laboratory's multidisciplinary team approach to science and technology. Safeguarding the nation from terrorist activity and the proliferation of weapons of mass destruction will be an enduring mission of this Laboratory, for which LDRD will continue to play a vital role.

The LDRD Program is a success story. Our projects continue to win national recognition for excellence through prestigious awards, papers published in peer-reviewed journals, and patents granted. With its reputation for sponsoring innovative projects, the LDRD Program is also a major vehicle for attracting and retaining the best and the brightest technical staff and for establishing collaborations with universities, industry, and other scientific and research institutions. By keeping the Laboratory at the forefront of science and technology, the LDRD Program enables us to meet our mission challenges, especially those of our ever-evolving national security mission. 


\section{Table of Contents}

About the FY2006 Laboratory Directed Research and Development Annual Report .......... 2

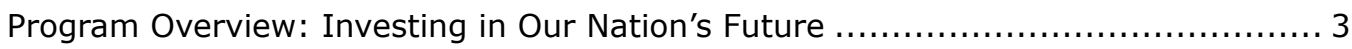

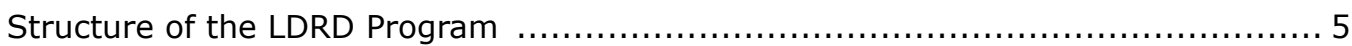

The LDRD FY2006 Portfolio: Investing in Our Nation's Future.......................... 7

\section{Section 1-Advanced Sensors and Instrumentation}

Ultrafast Transient-Recording Enhancements for Optical-Streak Cameras.................. 20

An Integrated Laboratory for the Study of Interventional Device Dynamics ................ 21

Developing Radiography for Advanced Radiography Capability at

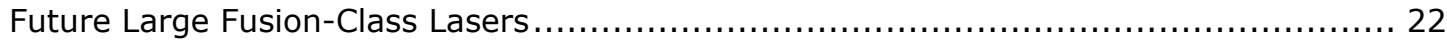

Nanobarometers: An in situ Diagnostic for High-Pressure Experiments $\ldots \ldots \ldots \ldots \ldots \ldots \ldots \ldots \ldots \ldots \ldots \ldots \ldots \ldots$

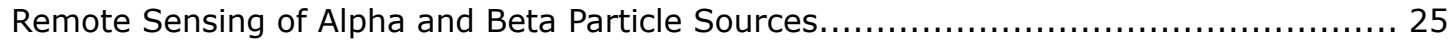

A Multiplexed Diagnostic Platform for Point-of-Care Pathogen Detection $\ldots \ldots \ldots \ldots \ldots \ldots \ldots \ldots \ldots \ldots \ldots \ldots$

Rapid Screening of Human Effluents with Single-Particle Mass Spectrometry

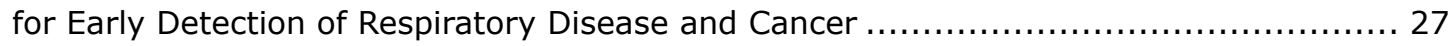

Probing Other Solar Systems with Current and Future Adaptive Optics ...................... 28

RadTracker: Optical Imaging of High-Energy Radiation Tracks ............................ 30

Amplifier and Compressor Technology for Split-Beam, High-Energy

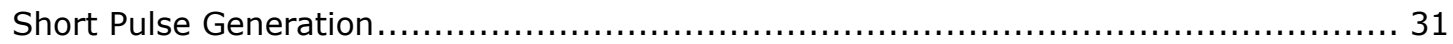

Leading the Quantum Limit Revolution ................................................... 32

Terascope: Terahertz Spectroscopic Imaging for Standoff Detection of

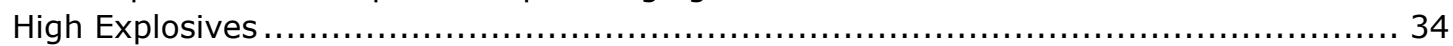

Rapid Defense Against the Next-Generation Biothreat.................................. 35

Nonclassical Noise Reduction for Sensing Applications................................ 36

Biophysical Characterization of Pathogen Invasion.................................... 37

Thermal-Fluidic System for Manipulating Biomolecules and Viruses....................... 38

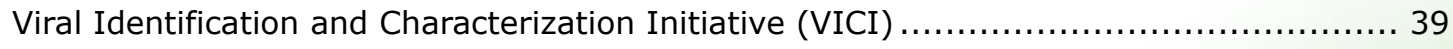

Real-Time, Ellipsometry-Based Transmission Ultrasound Imaging $\ldots \ldots \ldots \ldots \ldots \ldots \ldots \ldots \ldots \ldots \ldots \ldots \ldots \ldots \ldots \ldots$

\section{Section 2-Biological Sciences}

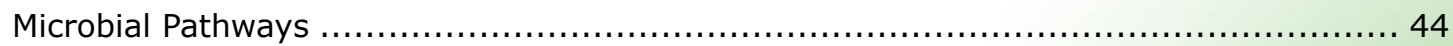

Force Spectroscopy to Study Multivalent Binding in Protein-Antibody Interactions ........ 45

Intracellular Chemical Measurements: A Generalized Approach with High Spatial Resolution Using Functionalized Nanoparticles.............................. 47

Using Femtosecond Laser Subcellular Surgery as a Tool to Study Cell Biology ............. 48 
Multiprobe Investigation of Proteomic Structure of Pathogens

Developing New Tools for in vivo Generation and Screening of Cyclic

Peptide Libraries.

A Coupled Computational and Experimental Approach to Determine

Functions of Deeply Conserved "Anonymous" Human Genes....

Characterizing the Regulatory Genome: Transcription Factor Proteins and

Gene Regulation Networks in Living Cells

De Novo Identification of Regulatory Regions in Intergenic Spaces

of Prokaryotic Genomes....

Time-of-Flight, Secondary Ion Mass Spectrometry Measurement of Metabolites from Single Cells .....

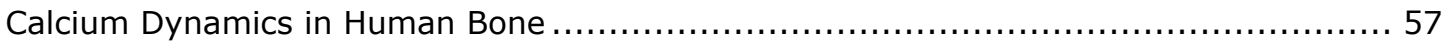

Single-Cell-Level Investigation of Cytoskeletal Response to External Stimuli ................ 58

A Single-Molecule Study of the Movement of a DNA Sliding Clamp .........................6 60

Emerging Contaminants: Application of Microarray Technology to the Detection of Mixtures of Endocrine-Active Agents

An Innovative Copolymer Complex to Inhibit Transport of Biological Aerosols

Characterizing Hypothetical Proteins

Comparative Analysis of Genome Composition with Respect to

Metabolic Capabilities and Regulatory Mechanisms

Development of a Chemoenzymatic-Like and Photoswitchable Method

for the Ordered Attachment of Proteins to Surfaces...

Developing and Integrating Novel Technologies for the Production

and Characterization of Membrane Proteins

Development of Single-Cell Raman Spectroscopy for Cancer Screening

and Therapy Monitoring....

Francisella Tularensis: Understanding the Host-Pathogen Interaction .................... 72

Characterization and Quantification of Dynamic Robustness in Biological Systems ......... 73

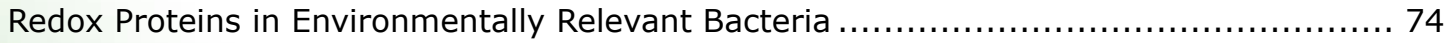

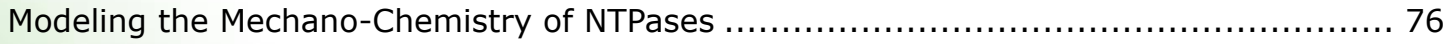

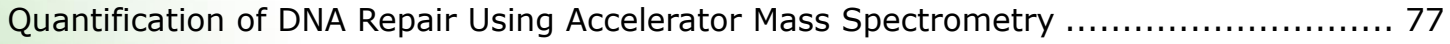

Developing a New Accelerator Mass Spectrometry Assay for Quantitation

of Platinum-DNA Adducts for Response to Platinum-Based Chemotherapy ................ 78

Analysis of the Mucin Membrane Protein by Cryo-Electron Microscopy

and Computational Image Processing.... 


\section{Section 3-Chemistry}

XChem 84

Bioforensics: Characterization of Biological Weapons Agents by NanoSIMS ................ 85

Ionization Chemistry of High-Temperature Molecular Fluids .............................. 86

New Fragment Separation Technology for Superheavy Element Research ................. 88

Heterogeneous Processes at the Intersection of Chemistry and Biology $\ldots \ldots \ldots \ldots \ldots \ldots \ldots \ldots \ldots$

Avoiding Surprise: Countering Novel Chem-Bio-Warfare Agent Threats .................... 91

Discovering the Folding Rules that Proteins Obey ......................................... 92

Conversion of Plutonium and Enriched Uranium .......................................... 94

Long-Time-Scale Shock Dynamics of Reactive Materials ................................. 95

Biologically Driven Fabrication of Complex Nanostructures at

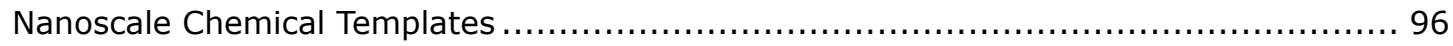

\section{Section 4-Earth and Space Sciences}

Nonaqueous-Phase Liquid Dissolution in Porous Media: Multiscale

Effects of Dissolution Kinetics on Cleanup Time............................................. 100

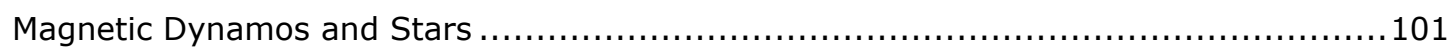

Dynamic Data-Driven Event Reconstruction for Atmospheric Releases.....................102

Coupling Micromechanics and Reactive Fluid Flow in Fracture Networks................. 104

Creating the Core Conditions of Extrasolar and Solar Giant Planets

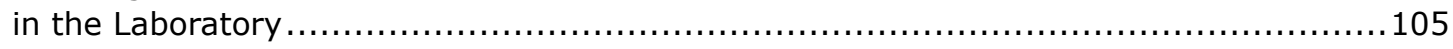

The Large Synoptic Survey Telescope and Foundations for Data

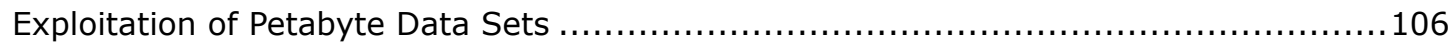

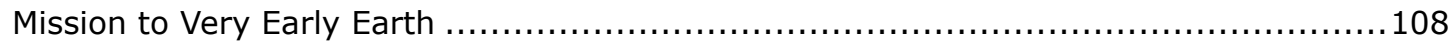

Iodine-129 Accelerator Mass Spectrometry for Earth Science,

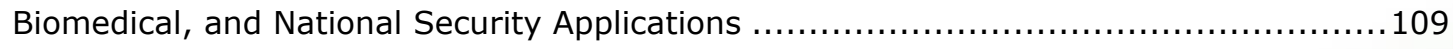

Carbon Flux in a California Grassland Soil Sequence: The Role of

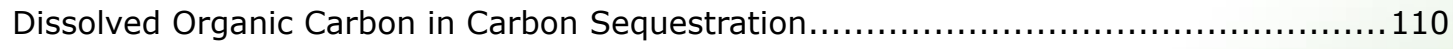

A New Capability for Regional, High-Frequency Seismic Wave Simulation in

Realistic Three-Dimensional Earth Models to Improve Nuclear Explosion Monitoring ......112

CHEMTREAT: Accelerated Remediation of Contaminated Fine-Grained

Sediments by a Chemical Clay Cracking and Co-Solvent Flushing Process.................113

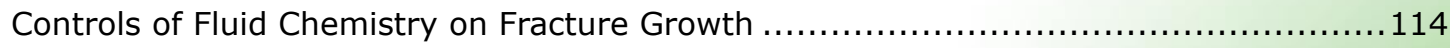

Integration and Codevelopment of a Geophysical Carbon Dioxide Monitoring Suite .......115

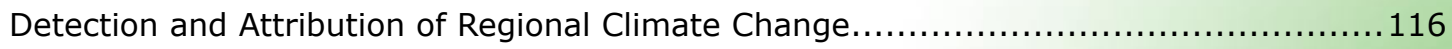


A Dynamically Coupled Groundwater, Land Surface, and Regional Climate Model to Predict Seasonal Watershed Flow and Groundwater Response

Developing a Reactive Chemistry Capability for the NARAC

Operational Model (LODI)

Enhanced Isolation Performance of Geologic Carbon Dioxide Storage Sites through

Mineral Trapping: Experimental and Field Confirmation of Model Predictions ...............120

The Physics of Recombining Plasmas in Celestial Sources

Urban Atmospheric Turbulence: Improved Turbulence Closure Models

through Observations and Simulations.

Atmospheric Carbon-14 Dioxide Constraints on and Modeling of Net

Carbon Fluxes....

Regional Climate

Development of Integrated Microanalysis of Nanomaterials

The Chemistry of Core Formation

Evidence for Stratospheric Downwelling Associated with

High-Elevation Topography

Dielectric Properties of Oil Shale

Thermal Diffusivity and Conductivity Measurements in Diamond Anvil Cells

\section{Section 5-Energy Supply and Use}

Persistent Monitoring Platforms

Environmental Consequences of Large-Scale Deployment of New

Energy Systems

Separation of Carbon Dioxide from Flue Gas Using Ion Pumping

\section{Section 6-Engineering and Manufacturing Processes}

Acoustic Characterization of Mesoscale Objects

Understanding and Improving High-Voltage Vacuum Insulators for

Microsecond Pulses

A Compact, High-Intensity Neutron Source Driven by Pyroelectric Crystals 144

Transport Behavior and Conversion Efficiency in Pillar-Structured

Neutron Detectors

\section{Section 7-Materials Science and Technology}

A Two-Particle Formulation of Electronic Structure 148

Plutonium and Quantum Criticality

High-Strain-Rate Deformation of Nanocrystalline Metals

Nanosecond Ultrasonics to Study Phase Transitions in Solid and Liquid

Systems at High Pressure and Temperature. 
Multiscale Characterization of Body-Centered-Cubic Crystals Deformed

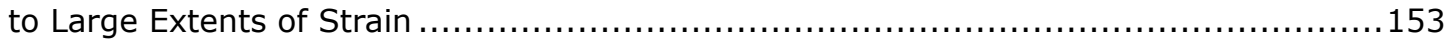

NanoBIS Determination of the Unoccupied Electronic Structure of Plutonium ..............155

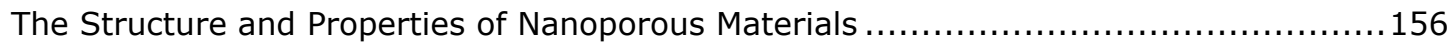

Characterization and Control of Laser-Induced Modifications in KDP and

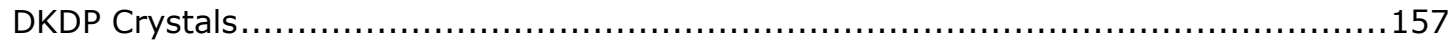

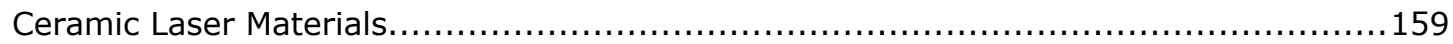

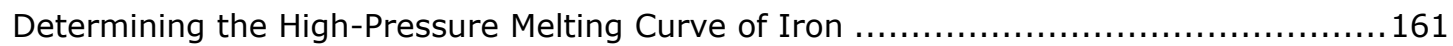

Mitigation of Optical Damage Sites on Ultraviolet Optics................................... 162

A Fracture Mechanics and Tribology Approach to Understanding Subsurface

Damage of Fused Silica During Grinding and Polishing .................................... 164

Characterization of the Effect of Short-Pulse Laser Exposure on Damage Size,

Morphology, and Conditioning in Wide-Bandgap Materials ..................................... 165

Molecular Transport in One-Dimensional Lipid Bilayers: A

Biological "Smoke Alarm" ...................................................................... 167

Target Fabrication Science and Technology: An Enabling Strategic Initiative ................169

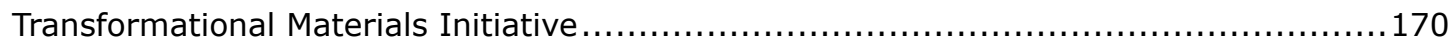

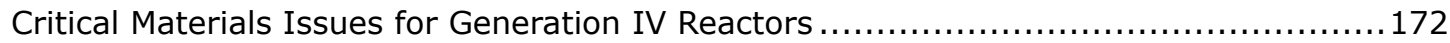

Time-Resolved Phase Transitions via Dynamic Transmission Electron Microscopy ..........173

Fundamental Investigation of Laser-Induced Surface Damage in Optical Materials ........175

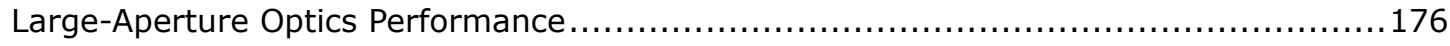

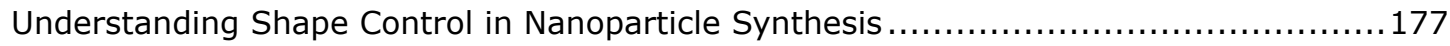

Enhanced Efficiency Chip Slapper for Detonator Applications Using a

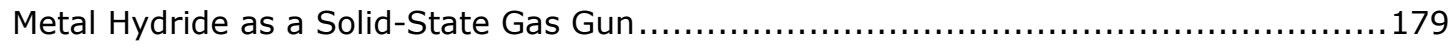

\section{Section 8-Mathematics and Computing Sciences}

Biological and Synthetic Nanostructures Controlled at the Atomistic Level ..................182

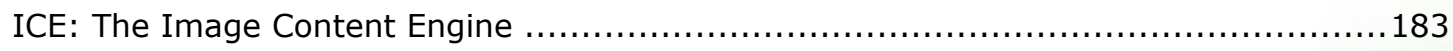

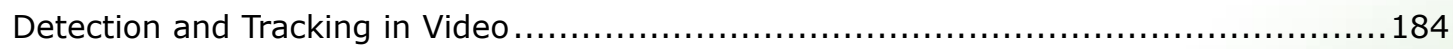

Three-Dimensional Vectorial Time-Domain Computational Photonics .........................185

Locally Adaptive Mesh Refinement for Linearly Scaling Electronic

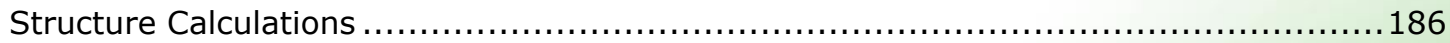

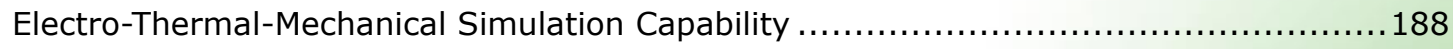

A New "Natural Neighbor" Meshless Method for Modeling Extreme

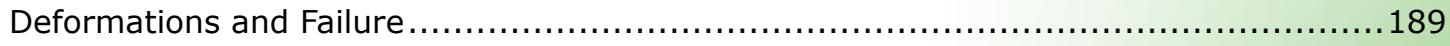

Internet Ballistics: Identifying Internet Adversaries Despite

Falsified Source Addressing.... 
Petascale Simulation Initiative

Catalyzing the Adoption of Software Components

LOCAL: Locality-Optimizing Caching Algorithms and Layouts

Risk Analysis of Secure Knowledge Discovery

A New Method for Wave Propagation in Elastic Media....

Efficient and Reliable Data Exploration via Multiscale Morse Analysis and Combinatorial Information Visualization

Predictive Knowledge Systems for Large, Complex Data Sources....

Development of Computational Techniques for Decoding the Language of Genomes

Scalable Data Management for Massive Semantic Graphs....

A Predictive Model of Fragmentation Using Adaptive Mesh Refinement and Hierarchial Material Model....

Decomposition of Large-Scale Semantic Graphs via an Efficient

Communities Algorithm ....

Image Relational Search Engine

Data Intensive Computing .....

A Novel Structure-Driven Approach to Sequence Pattern Definition for

Remote Homology Detection

Multi-Petabyte Image Data Management Systems

Quantum Monte Carlo Assessment of the Relevance of Electronic

Correlations in Defects and Equations of State in Metals

Diffusion Monte Carlo without All the Hops

\section{Section 9-Nuclear Science and Engineering}

Broadband Radiation and Scattering.

Decontamination of Terrorist-Dispersed Radionuclides from Surfaces

in Urban Environments...

A Coupled, Multiphysics Code for Accurate Modeling of Nuclear Reactors

\section{Section 10-Physics}

Laser-Matter Interactions with a 527-Nanometer Drive

Characterization and Optimization of High-Energy K-Alpha X-Ray Sources ......

Development of Absolute Spectroscopic Diagnostics for

Nonlocal-Thermodynamic-Equilibrium Plasmas.

Electronic Transitions and Phonons in f-Band Metals at High Pressures

Short-Pulse Laser Absorption and Energy Partition at Relativistic

Laser Intensities. 
The Creation of a Neutron Star Atmosphere .... 230

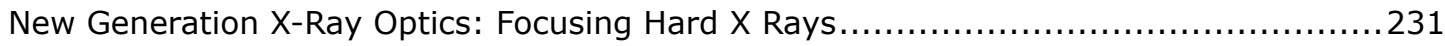

Nanomechanics: Strength and Structure for Nanotechnology ............................232

High-Average-Power, High-Energy, Short-Pulse Fiber Laser System ........................234

Development and Application of a Predictive Computational Tool for Short-Pulse, High-Intensity Target Interactions.......................................... 235

Surrogate Nuclear Reactions and the Origin of Heavy Elements...........................237

Stellar Astrophysics and a Fundamental Description of

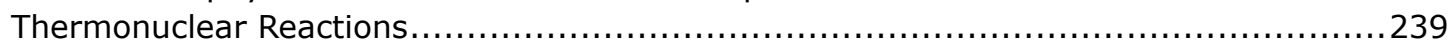

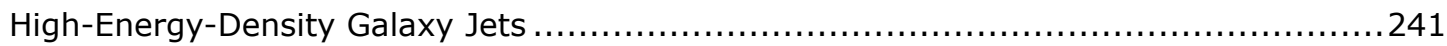

High-Brightness, Laser-Driven, X-Ray Source for Nanoscale Metrology

and Femtosecond Dynamics ................................................................. 242

Ultrafast, in situ Probing of Shocked Solids at the Mesoscale and

Beyond: A New Paradigm for Materials Dynamics........................................... 243

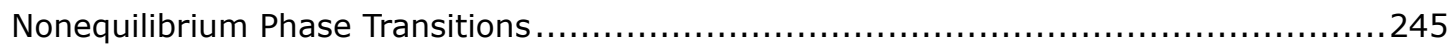

Kinetic Simulation of Boundary-Plasma Turbulent Transport..............................246

Biological Imaging with Fourth-Generation Light Sources ................................. 248

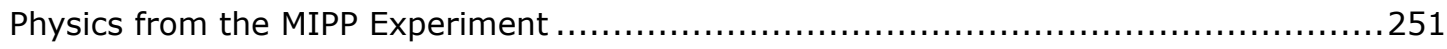

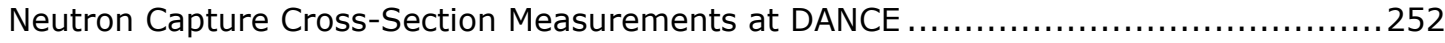

Hysteresis and Kinetic Effects during Liquid Solid Transitions .............................253

Optical Properties as a Real-Time in situ Materials Diagnostic at

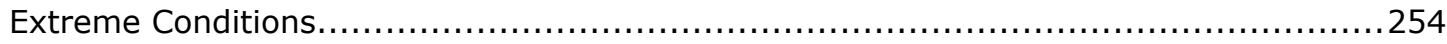

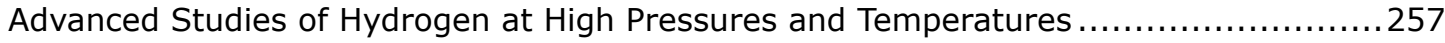

Hydrodynamic, Atomic Kinetic, and Monte Carlo Radiation Transfer Models

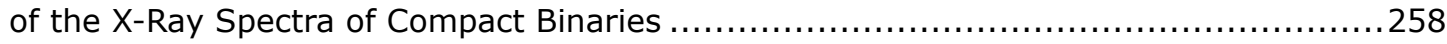

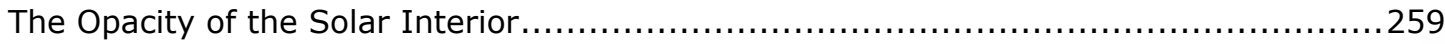

Split-Beam, Short-Pulse Final Optics and Characterization for High-Energy

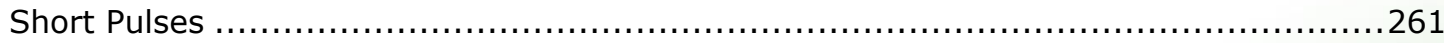

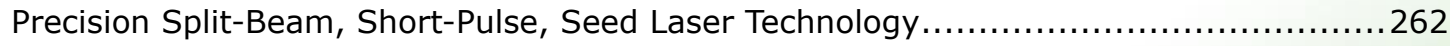

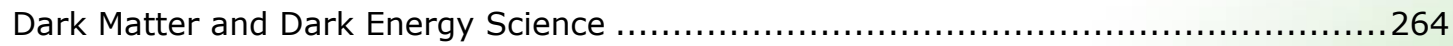

Development of Hot, LTE-Tunable Radiation Sources for Material Science

Studies and Simulating Radiation Transport in Dense Astrophysical Plasmas ...............265

Developing the Physics Basis of Fast-Ignition Experiments at

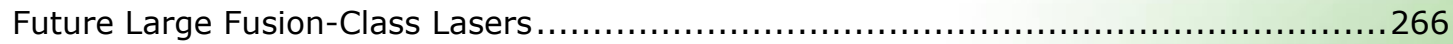

Measuring Plasmon Density of States in Dense Matter .................................. 268 
Understanding the Nuclear Magnetic Fields .270

High-Pressure, Multimegabar Conductivity Experiments on Hydrogen:

The Quest for Solid Metallic Hydrogen

Novel High-Energy-Density Source

Active Detection and Imaging of Nuclear Materials with High-Brightness

Gamma Rays

The Ultrafast Lattice Response of the Shocked Solid ......................................276

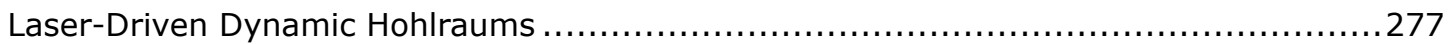

High-Resolution Measurements of the Plasma Current Profile for ITER …..................279

Investigating New Regimes of Material Strength at Ultrahigh Strain

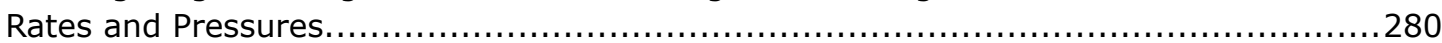

The Properties of Confined Water and Fluid Flow at the Nanoscale .........................281

Spheromak Energy Transport Studies via Neutral Beam Injection .........................282

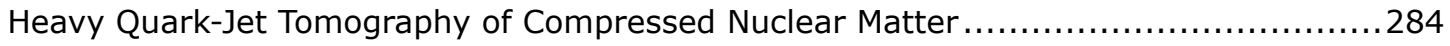

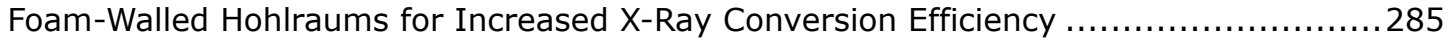

Mitigation of Electromagnetic Pulse Effects from Short-Pulse Lasers and

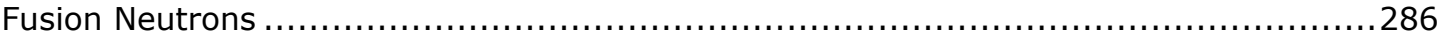

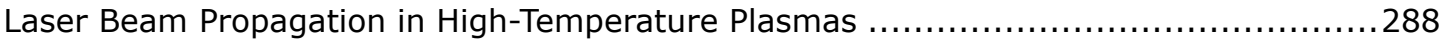

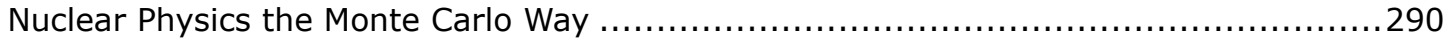

Observation of Coherent Terahertz Frequency Emission from Shocked

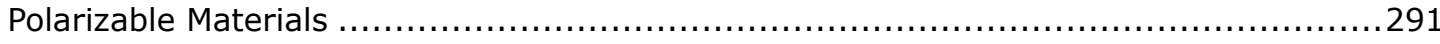

Feasibility Study for Large, Water-Based Neutron and Neutrino Detectors.................293

Feasibility of Gratings for Increasing Solid-State Laser Gain and Efficiency ................294 
Laboratory Directed Research and Development

Overview 


\section{About the FY2006 Laboratory Directed Research and Development Annual Report}

The FY2006 Laboratory Directed Research and Development (LDRD) Annual Report provides a summary of LDRD-funded projects for the fiscal year and consists of two parts:

Overview: An introduction to the LDRD Program, the LDRD portfolio-management process, program statistics for the year, and highlights of accomplishments for the year.

Project Summaries: A summary of each project, submitted by the principal investigator. Project summaries include the scope, motivation, goals, relevance to Department of Energy/National Nuclear Security Administration and Lawrence Livermore National Laboratory mission areas, the technical progress achieved in FY2006, and a list of publications that resulted from the research in FY2006.

Summaries are organized in sections by research category (in alphabetical order). Within each research category, the projects are listed in order of their LDRD project category: Strategic Initiative (SI), Exploratory Research (ER), Laboratory-Wide Competition (LW), and Feasibility Study (FS). Within each project category, the individual project summaries appear in order of their project tracking code, a unique identifier that consists of three elements. The first is the fiscal year the project began, the second represents the project category, and the third identifies the serial number of the proposal for that fiscal year. For example:

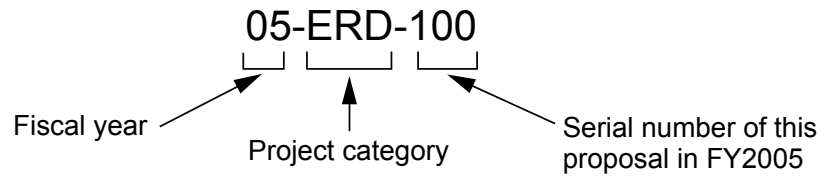




\section{Program Overview: Investing in Our Nation's Future}

\section{About Lawrence Livermore National Laboratory}

A premier applied-science laboratory, Lawrence Livermore National Laboratory (LLNL or the Laboratory) has at its core a primary national security mission - to ensure the safety, security, and reliability of the nation's nuclear weapons stockpile without nuclear testing, and to prevent and counter the spread and use of weapons of mass destruction: nuclear, chemical, and biological.

The Laboratory uses the scientific and engineering expertise and facilities developed for its primary mission to pursue advanced technologies to meet other important national security needs-homeland defense, military operations, and missile defense, for examplethat evolve in response to emerging threats. For broader national needs, the Laboratory executes programs in energy security and long-term energy needs, environmental assessment and management, bioscience and technology to improve human health, and breakthroughs in fundamental science and technology. With this multidisciplinary expertise, the Laboratory serves as a science and technology resource to the U.S. government and as a partner with industry and academia.

One of three Department of Energy (DOE)/National Nuclear Security Administration (NNSA) laboratories, LLNL has been managed since its inception in 1952 by the University of California (UC). This half-century association with UC has enabled the Laboratory to establish an atmosphere of intellectual freedom and innovation that attracts and maintains the world-class workforce needed to meet challenging national missions.

\section{Laboratory Directed Research and Development Program}

To fulfill its missions, LLNL must continually invest in the science and technology that form the foundation of its signature capabilities. The Laboratory Directed Research and Development (LDRD) Program, which was established by Congress at all DOE national laboratories in 1991, is LLNL's most important single resource for fostering excellent science and technology for today's needs and tomorrow's challenges.

According to its Congressional mandate ${ }^{1}$ the purpose of LDRD is to foster excellence in science and technology that (1) supports the DOE/NNSA and LLNL missions and strategic vision, (2) ensures the technical vitality of the Laboratory, (3) attracts and maintains the most qualified scientists and engineers and allows scientific and technical staff to enhance their skills and expertise, (4) helps meet evolving DOE/NNSA and national security needs, and (5) enables scientific collaborations with academia, industry, and other government laboratories.

By enabling LLNL to fund creative basic and applied research activities in areas aligned with its missions, the LDRD Program develops and extends the Laboratory's intellectual foundations and maintains its vitality as a premier research institution. The present scientific and technical strengths of LLNL are, in large part, a product of LDRD investment choices in the past.

1 U.S. Department of Energy. Order 413.2A. Laboratory Directed Research and Development (January 8,2001 ) 
2 U.S. Department of Energy (2003). Strategic Plan. http:// strategicplan.doe.gov/ (retrieved February 5, 2005).

\section{The LDRD Portfolio Management Process}

The FY2006 LDRD portfolio-management process at LLNL consisted of three major components that ensured the quality of the year's portfolio and its alignment with the DOE/ NNSA and the Laboratory's missions: (1) a top-level strategic planning process to identify strategic science and technology areas for LDRD investment, (2) a call to the Laboratory scientific and technical community for innovative and relevant proposals within the DOE/NNSA mission areas, and (3) a scientific peer-review process to select the highest quality LDRD portfolio from these proposals.

In FY2006, the top-level LDRD strategic planning process was guided by the DOE Strategic $P{ }_{a n}{ }^{2}$ for the next 25 years, and by the Laboratory's own long-range plan that will define the scientific and technical strategy for the coming decade. The 2004 DOE Strategic Plan articulates four strategic goals for achieving the DOE mission of advancing the national, economic, and energy security of the U.S.; promoting scientific and technological innovation in support of that mission; and ensuring the environmental cleanup of the national nuclear weapons complex. In FY2006, the Laboratory's LDRD Program strongly supported all four DOE strategic goals:

1. Defense-Protect our national security by applying advanced science and nuclear technology to the nation's defense.

2. Energy-Protect our national and economic security by promoting a diverse supply and delivery of reliable, affordable, and environmentally sound energy.

3. Science-Protect our national and economic security by providing world-class scientific research capacity and advancing scientific knowledge.

4. Environment-Protect the environment by providing a responsible resolution to the environmental legacy of the Cold War and by providing for the permanent disposal of the nation's high-level radioactive waste.

The Laboratory's Long-Range Strategic Science and Technology (S\&T) Plan continues to guide the LDRD portfolio planning process. Broadly inclusive, the Laboratory's S\&T Plan is intended to elicit the most far-reaching and innovative ideas for the future shape of science and technology at LLNL. The six thematic areas of the S\&T Plan are as follows:

- Stockpile science and technology.

- High-energy-density science and technology.

- Nuclear, radiative, and astrophysical science and technology.

- Science and technology at the intersection of chemistry, biology, and materials science and technology.

- Information, simulations, and systems science and technology.

- Energy and environmental science and technology (with fusion energy science and technology as a special subtopic). 
The NNSA oversees the Laboratory's LDRD Program to ensure that it accomplishes its objectives. This oversight includes field and headquarters reviews of both the technical content and management processes. As demonstrated in a memorandum (April 30, 2002) from the Secretary of Energy and the NNSA Administrator, the DOE/NNSA actively supports the LDRD Program. In the memo, Secretary of Energy Spencer Abraham writes: "I believe that all sponsors, including other federal agencies, benefit from the strong science and technology base provided by the Department's [LDRD] programs. [LDRD] is at the core of our ability to develop research capabilities and apply advanced technologies to effectively meet the Department's and the nation's needs." ${ }^{\prime 3}$

\section{Structure of the LDRD Program}

\section{Project Categories}

The LDRD Program at LLNL consists of three major project categories: Strategic Initiative (SI), Exploratory Research (ER), and Laboratory-Wide (LW) competition. Throughout the year, the LDRD Program also funds a few projects in a fourth category, Feasibility Study/ Project Definition (FS).

\section{Strategic Initiative}

The SI category focuses on innovative R\&D activities that are likely to set new directions for existing programs, help develop new programmatic areas within LLNL's mission responsibilities, or enhance the Laboratory's science and technology base. Projects in this category are usually larger and more technically challenging than projects funded in other categories. An SI project must be aligned with the strategic R\&D priorities of at least one of the six thematic areas of LLNL's Long-Range Strategic S\&T Plan.

\section{Exploratory Research}

The ER category is designed to help fulfill the strategic R\&D needs of a Laboratory directorate (ERD) or institute (ERI). In this category, researchers submit proposals to their directorates and institutes, where the proposals are screened and subsequently forwarded to the ER selection committee for review. In FY2006, LLNL's S\&T Plan continued to guide directorates in evaluating the ERD and ERI proposals.

\section{Laboratory-Wide Competition}

Projects in the LW category emphasize innovative research concepts and ideas and undergo limited management filtering. The LW competition is open to all LLNL staff in programmatic, scientific, engineering, and technical support areas. Researchers submit their project proposals directly to the LW selection committee.

\footnotetext{
${ }^{3}$ Memorandum from Secretary of Energy Spencer Abraham. 2002-0077386 (Washington, DC, April 30, 2002).
} 


\section{Feasibility Study/Project Definition}

This special project category, FS, provides researchers with the flexibility to define and develop potential projects in the other three categories. To increase its responsiveness to Laboratory scientists and engineers, the LDRD Program funds FS projects throughout the year.

\section{Project Competency Areas}

Although LDRD projects often address more than one scientific discipline, each project is classified into one of ten research categories that is relevant to NNSA and Laboratory missions. The ten categories are:

- Advanced Sensors and Instrumentation.

- Biological Sciences.

- Chemistry.

- Earth and Space Sciences.

- Energy Supply and Use.

- Engineering and Manufacturing Processes.

- Materials Science and Technology.

- Mathematics and Computing Sciences.

- Nuclear Science and Engineering.

- Physics. 


\section{The LDRD FY2006 Portfolio: Investing in Our Nation's Future}

\section{Overview of the FY2006 Portfolio}

The FY2006 LDRD portfolio was carefully structured to continue the LDRD Program's vigorous support for the strategic vision and long-term goals of DOE/NNSA and LLNL. The FY2006 projects described in this Annual Report underwent a stringent selection process and received ongoing management oversight.

In FY2006 the LDRD Program funded 188 projects with a total budget of $\$ 92.6$ million. Figure 1 shows the number of projects in each of the four categories. Figure 2 shows the distribution of funding among the four LDRD project categories.

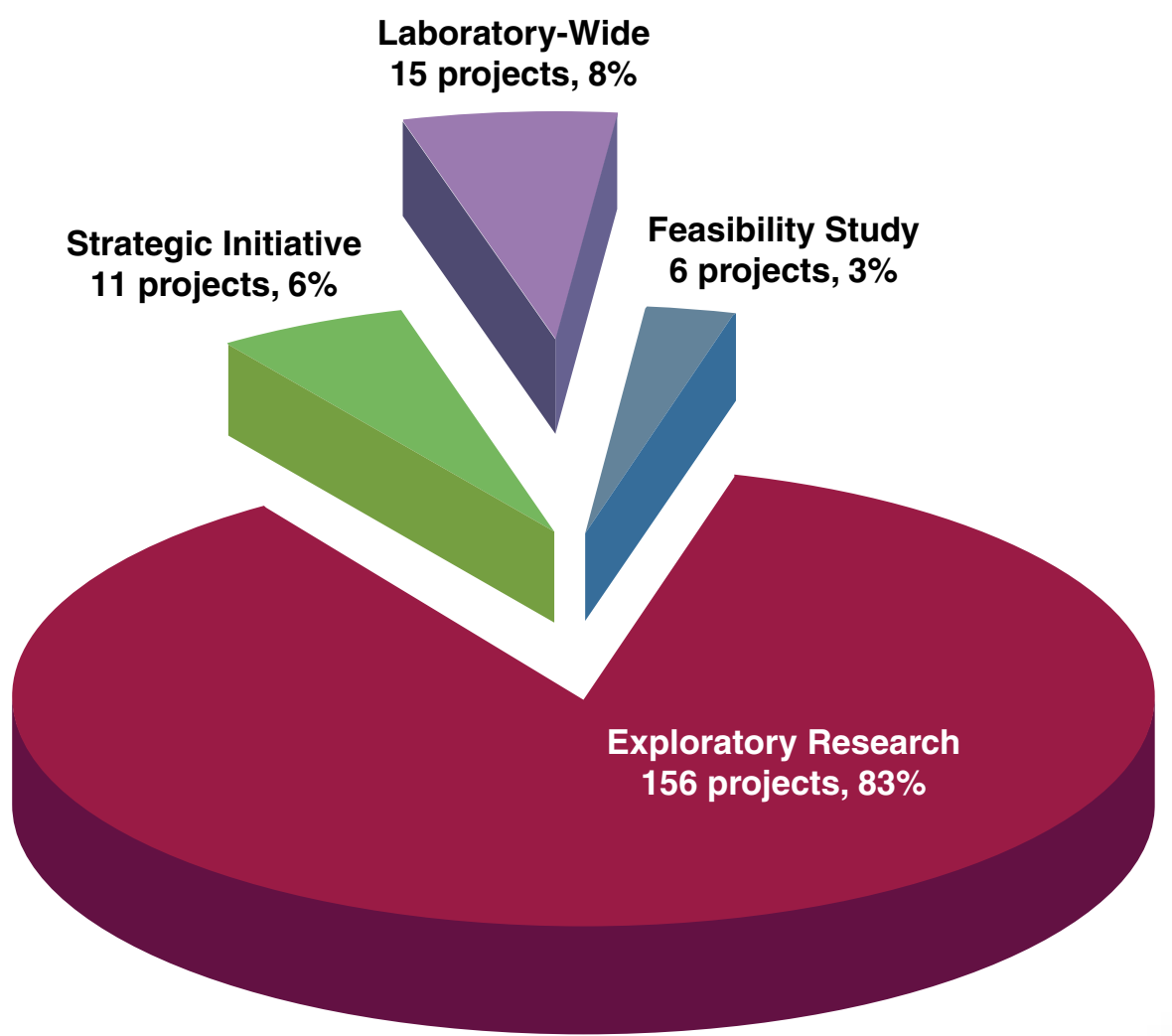

Figure 1. Number and percentage of the 188 LDRD projects in each project category in FY2006. 


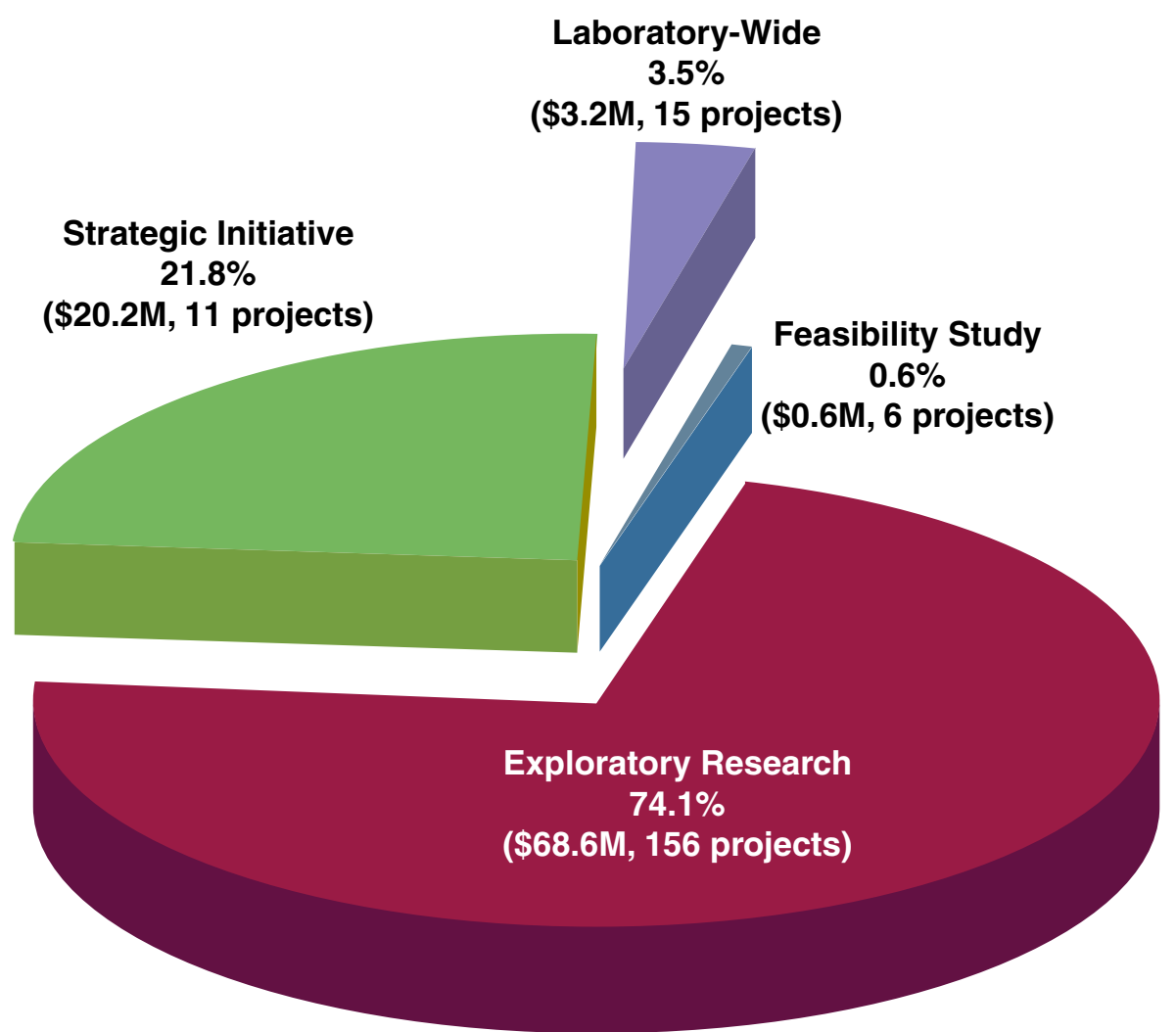

Figure 2. Distribution of funding among the four LDRD project categories. Total funding for FY2006 was \$92.6M.

\section{Strategic Initiative}

In FY2006, the LDRD Program funded 11 SI projects. Although the SI category represented only $6 \%$ of the total number of LDRD projects for FY2006, it accounted for $21.8 \%$ of the budget. SI projects ranged in funding from $\$ 289.3 \mathrm{~K}$ to $\$ 3.4 \mathrm{M}$.

\section{Exploratory Research}

The LDRD Program funded 156 ER projects for FY2006. The largest project category, ERs accounted for $83 \%$ of LDRD projects for the fiscal year. Projects in this year's ER category ranged in budget from $\$ 16 \mathrm{~K}$ to $\$ 3.4 \mathrm{M}$. 


\section{Laboratory-Wide Competition}

In 2006, 15 LW projects were funded, which represent $8 \%$ of the LDRD projects for the year and $3.5 \%$ of the budget. LW projects are limited to $\$ 190 \mathrm{~K} /$ year funding, with a few exceptions. In FY2006, LW projects ranged in funding level from $\$ 142.7 \mathrm{~K}$ to $\$ 264 \mathrm{~K}$.

\section{Feasibility Study}

The LDRD Program funded six FS projects for FY2006, or 3\% of the total. FS projects are limited to $\$ 75 \mathrm{~K}$ and a 12 -month duration.

Figure 3 shows the funding distribution by dollar amount for the 188 FY2006 projects$85 \%$ of the projects were in the $\$ 101 \mathrm{~K}$ to $\$ 500 \mathrm{~K}$ range, with $6.9 \%$ falling below $\$ 100 \mathrm{~K}$. This lowest funding level includes all the FS projects. Projects in the $\$ 501 \mathrm{~K}$ to $\$ 1 \mathrm{M}$ funding range accounted for $7.4 \%$ of the total, and another $7.4 \%$ of the projects received more than $\$ 1 \mathrm{M}$. The average funding level for the 188 projects was $\$ 493 \mathrm{~K}$.

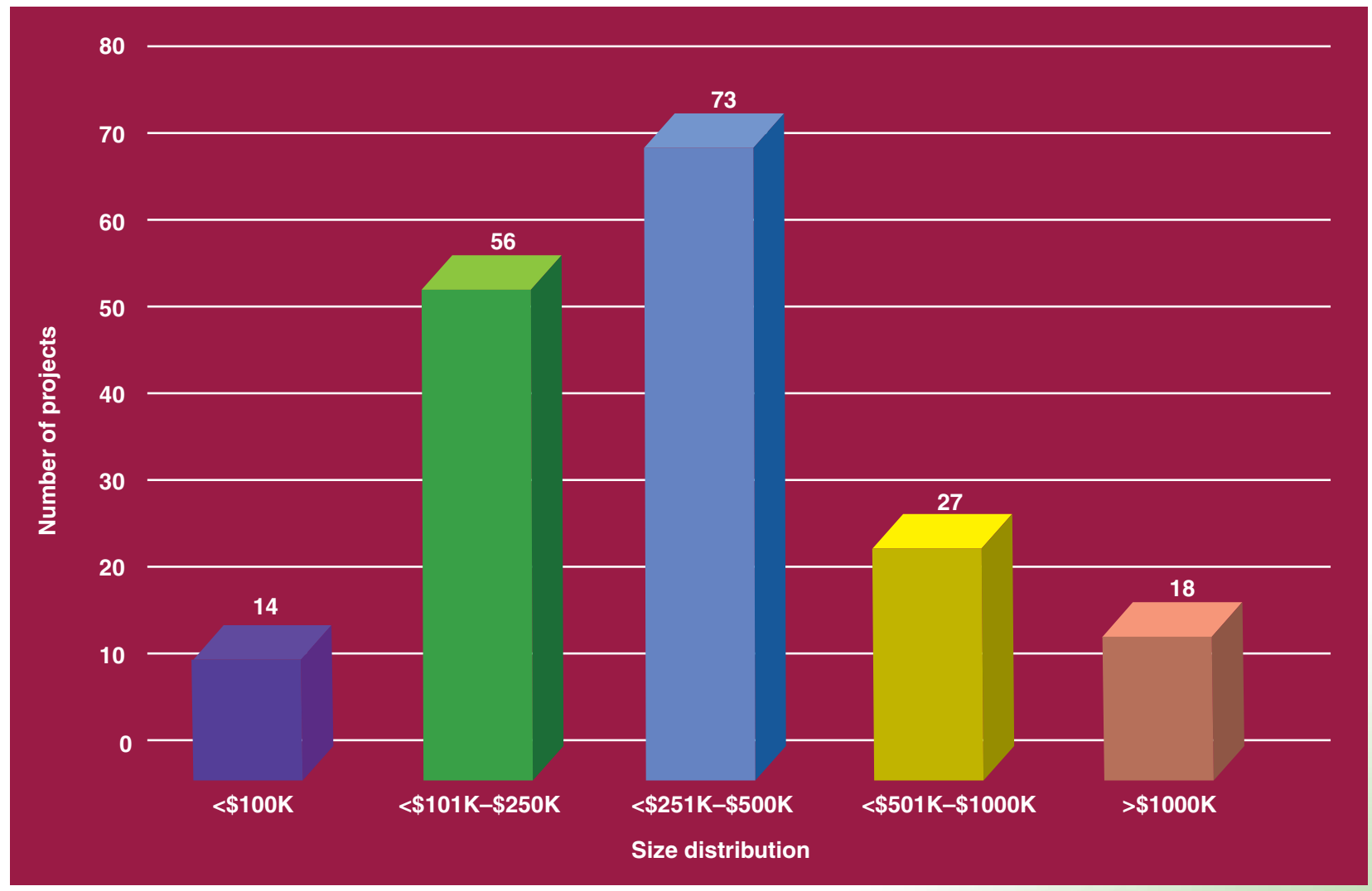

Figure 3. Number of projects and levels of funding. The average funding level for an LDRD project in FY2006 was \$493K. 


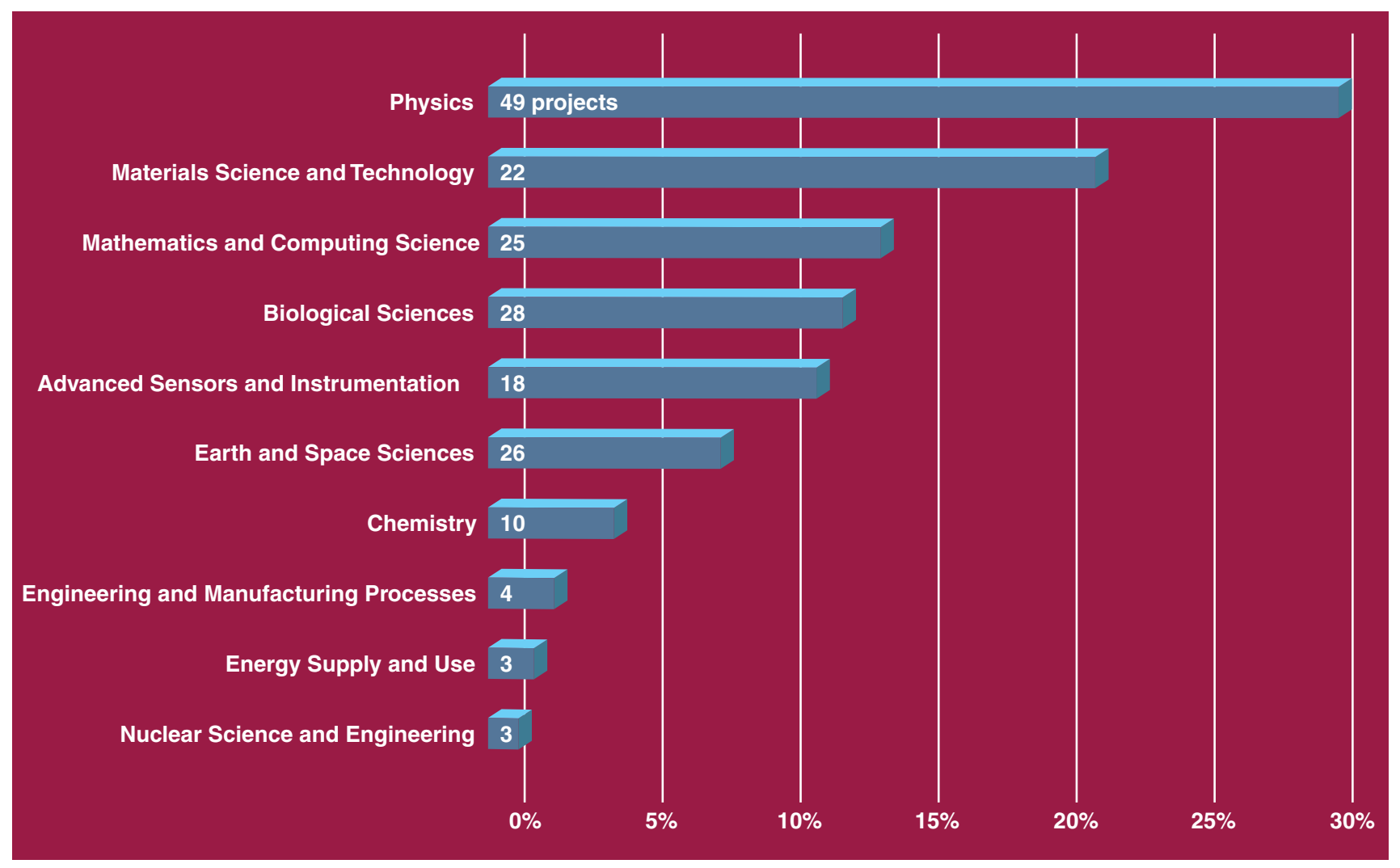

Figure 4. Percentage of LDRD funding and number of projects in each research category in FY 2006.

Figure 4 shows the percentage of LDRD funding and number of projects in each research category for FY2006.

\section{Highlights of FY2006 LDRD Accomplishments}

In FY2006, the LDRD Program at LLNL continued to be extremely successful in achieving its goals of scientific discovery, providing new concepts for core missions, and creating an exciting research environment that attracts outstanding young talent to the Laboratory. Below is a selection of FY2006 highlights that exemplify LDRD's noteworthy research results, timely support for critical national needs, and external recognition of Laboratory personnel.

\section{- A New Method for Wave Propagation in Elastic Media (05-ERD-079)}

This project is producing a verified, accurate, and efficient elastic-wave-propagation code for numerical simulation of elastic-wave propagation in complex two- and threedimensional (2D and 3D) media. Such code is essential for monitoring nuclear explosions, characterizing underground facilities, predicting ground motion from earthquakes, and 
nondestructively evaluating complex parts. Other applications include strong groundmotion prediction for the Enhanced Test Site Readiness Program and the Yucca Mountain Program and locating imperfections in components relevant to the Transformative Materials Initiative (06-SI-005). This computer software will run on computers ranging from desktop workstations to massively parallel high-performance machines, such as Thunder and Atlas.

Efforts in FY2006 focused on large-scale simulations of the 1906 San Francisco earthquake as a means of validating the code. The team developed the capability to read the geologic model provided by the U.S. Geological Survey, perform robust discretization of rapidly fluctuating material properties, extend the elastic scheme to allow for acoustic regions (water), improve discretization of moment sources to allow a general 3D fault surface, remove solid-body motion using a projection technique, and interface a wavelet library for compressing volumetric data for 3D post-processing. In addition, this code was demonstrated to visitors at a San Francisco earthquake centennial event held at LLNL, and the project itself was highlighted at the NNSA tri-laboratory LDRD review in August 2006.

\section{- Development of Integrated Microanalysis of Nanomaterials (06-ERI-001)}

This project is developing a microanalysis capability that integrates the latest electron microscopy and ion microprobe techniques, combined with state-of-the-art instrumentation at LLNL, to enable a new level of investigations into the mineralogical, chemical, and isotopic properties of nanomaterials. This includes cometary particles captured at hypervelocity speeds in low-density silica aerogels by NASA's Stardust samplereturn capsule-the first solid-matter materials to be returned to Earth since the Apollo missions of the 1970s. Analysis of these particles will provide new insights into cosmically primitive extraterrestrial material, such as whether comets played an important role in bringing life to early Earth. Lessons learned from this project will also develop synergistic capabilities for extracting and analyzing nanoscale materials that are directly applicable to the Laboratory's missions in stockpile stewardship and homeland security, such as extracting particulate ejecta from aerogel after fusion-class laser experiments.

In FY2006, LLNL's Stardust team began extracting and analyzing particles collected from Comet Wild 2 and successfully returned to Earth by the Stardust capsule in January. The particles were extracted from silica aerogel and aluminum foil and analyzed using transmission electron microscopy, focused ion beam-field emission scanning electron microscopy, and synchrotron beamlines at the Stanford Synchrotron Radiation Laboratory and the Advanced Light Source. The Wild 2 particles were found to contain materials that have never been remotely detected in a comet, including calcium-aluminum inclusions. Because these inclusions solidify at several thousand degrees, their presence suggests they formed close to the young Sun and then were flung outward, meaning much more mixing occurred in the early solar system than was previously thought. These findings lend credence to theories such as the "X-wind" theory-in which the materials that eventually condensed into our solar system were propelled away from the young Sun by its strong magnetic field-and indicates that we must re-examine our theories of flow and mixing in the early solar nebula.

The December 15, 2006, issue of Science was a special issue devoted to the Stardust project. Coverage in the popular press was also considerable. John Bradley, the LDRD 
project's principle investigator, held a press conference at LLNL. Both he and co-investigator Hope Ishii appeared on several radio and TV programs after the analysis was announced. Hope's work related to Stardust was instrumental in her winning a 2006 Science Spectrum Magazine Top Minorities in Science "Trailblazer" Award.

\section{- Ceramic Laser Materials (05-ERD-037)}

Currently, slabs of single-crystal optical materials for high-power lasers are very difficult to grow, and the larger slab sizes that would increase available laser power may not be possible. The objective of this project is to evaluate the use, in high-power lasers, of slabs of transparent ceramics created by sintering powders at temperatures below their melting point. These ceramics can offer the same optical and thermal properties as single crystals but have advantages such as design flexibility, larger aperture capability, ease and robustness of manufacture, potential multifunctionality, higher and more uniform activator concentrations, and reduced brittleness. In collaboration with an industrial partner, this project is developing a robust process for making transparent ceramics with innovations such as novel methods for preparing the very pure starting nano-powders and methods of extrusion for forming the pre-sintered material. In addition, the project team is designing several laser architectures that are possible only with ceramic materials. Examples include large-aperture slabs for a solid-state heat-capacity lasers, which can provide the power needed for defense applications such as destroying mortar shells, rockets, and land mines.

In FY2006, the team optimized and validated its process for making transparent yttriumaluminum-garnet (YAG) ceramics, which were also damage tested. Optical quality was then significantly improved by adding a hot isostatic press step. Near transparency was also achieved in several other materials, including strontium fluoride. The team also fabricated samarium-doped YAG as an amplified-spontaneous-emission suppressor integrally sintered in a frame around neodymium-doped YAG ceramic. The spectra and lifetimes of ytterbium-yttrium oxide, a potential high-power laser-gain material, were also measured.

\section{- Transformational Materials Initiative (06-SI-005)}

This project will create new materials and technologies that will reduce the cost and time required to produce and maintain the nuclear stockpile, enhance weapon safety, ensure future stockpile longevity, and optimize stockpile performance. The ultimate goal is to provide the underlying science and technology for ensuring the continued success of the Stockpile Stewardship Program and for making the U.S. nuclear weapons complex smaller, safer, and more agile. The project's multidisciplinary team combines capabilities in materials synthesis, characterization, theory, and modeling to deliver cutting-edge advances in high explosives, sensing, metals, and multifunctional materials.

Using advanced computer simulations-including molecular dynamics, continuum dynamics, and density functional methods-the team in FY2006 developed a new class of solvents that allows for processing high explosives with optimal shock insensitivity, as well as novel polymers that were shown to improve the strength of processed explosives, two new composite alloys, and a new explosive method for material property tests. A novel nanoscopic material for the catalytic scavenging of hydrogen was developed, and in collaboration with five universities and two other weapons labs, the project team also 
narrowed eight candidate sensing technologies to three focus areas. Groundbreaking work done in FY2006 also includes exploring the use of ionic liquids as a new medium for making energetic crystals and the investigation, in collaboration with UC Berkeley, of carbon nanotube and nanowire gas sensors.

\section{- Diffusion Monte Carlo without All the Hops (06-LW-028)}

Simulations based on the diffusion Monte Carlo (DMC) technique are used in research relevant to stockpile science and technology-such as predictive modeling of plutonium aging-and to energy science and security-such as studying materials for fusion reactors. These simulations, which replicate events at an atomistic level, require massive computational resources. However, an age-old limitation of standard DMC is that in many cases, a large percentage of the molecules in a simulation are not directly involved in the event of interest. This project is developing a new method for DMC simulations that will eliminate this waste to perform simulations more quickly and utilize supercomputer resources more efficiently. Ultimately, this will open the door to new opportunities for the predictive modeling of complex processes that are relevant to Laboratory missions but for which standard DMC is ineffective. Based on the theory of first-passage processes, this approach will boost the performance of DMC simulations by orders of magnitude, by skipping most of the diffusion hops and projecting the system's state directly to collisions. The project team will implement the new method in an efficient computer code (in both sequential and parallel versions) convenient for a wide variety of applications relevant to Laboratory missions. The resultant computer code will be maintained as an open-source software and made available to computational scientists throughout the DOE complex.

In FY2006, the project team extended its first-passage kinetic Monte Carlo algorithm from discrete-lattice, one-dimensional (1D) diffusion to continuous-space, 3D diffusion using cubes for walker protection; wrote, debugged, and tested a FORTRAN code based on the new algorithm; conducted computational experiments showing that in comparison to the classic method, their new algorithm produces equivalent statistics of particle diffusion and far surpasses the classical algorithm in computational efficiency when the density of diffusing particles is low; and published a paper on the new DMC method in the journal Physical Review Letters. Finally, this project's principal investigator became a fellow of the American Physical Society partly because of this project's achievements.

\section{- New Fragment Separation Technology for Superheavy Element Research (04-ERD-085)}

This project is investigating the western edge of the "island of stability"-an unexplored high-mass region of the periodic table where elements are predicted to have particularly stable "magic numbers" of protons and neutrons. The goal is to determine whether or not the closed proton shell-which has profound implications for nuclear physics modelsis located at 114 protons. This will be accomplished by fabricating a thick plutonium ceramic target that will be bombarded with calcium-48 to produce element-114 isotopes, which will be separated in the Mass Analyzer for Super Heavy Atoms (MASHA) in Dubna, Russia to identify the atomic mass of the element-114 isotopes, and by irradiating californium-249; curium-245, 248; and americium-243 targets to produce elements 118,116 , and 115 , which will further define the edge of the island of stability. These experiments have already led to discovery of a new superheavy element (element 118) 
and are expected to better establish the decay properties of the isotopes of elements 115 and 113 and gather additional experimental evidence to help determine whether the island of stability is centered at 114 protons or at a higher atomic number. The results of these experiments will extend and improve theoretical models used to calculate the decay properties and nuclear shapes of the heaviest elements. Efforts such as this one, to synthesize new elements and measure their properties, furthers the Laboratory's competencies in nuclear chemistry and radiochemistry, which are relevant to assessing nuclear device performance and countering proliferation activities involving nuclear materials.

During FY2006, the team announced the discovery of element 118 and continued synthesizing ceramics at MASHA for a plutonium-244 target that will be used to produce element 114. They also investigated the possibility of in-beam tests of these targets at several facilities, developed a new chemical separation for element 105, found evidence that element behaves more like its homolog tantalum rather than niobium, and chemically isolated and identified the element-115 decay daughter. Irradiation of americium-243, curium-245, and californium-249 was also continued. This project's recent achievements were presented at several high-profile conferences, published in the journals Nature and Physical Review C, and featured in an episode of "NOVA Science Now." The discovery of element 118 was also named by Scientific American as one of the most important science stories of 2006.

\section{- Nonequilibrium Phase Transitions (04-ERD-108)}

This project is conducting the first systematic study of nonequilibrium phase transitions-a scientific frontier that holds promises for discovering new phases, metastable states, chemical reaction pathways, and biological functioning processes. Using measurements to correlate optical and structural properties under ultrafast laser excitation, the team is examining lattice disordering and melting, quantifying the role of electronic excitation on phase-transition kinetics, and developing approaches in finite-temperature condensed matter for constructing an equation of state (EOS). Quantum simulations based on the density functional theory approach will be benchmarked with time-correlated data on optical and structural properties by tracking solid-liquid to liquid-plasma transitions under ultrafast excitation conditions. Success of the project will lead to new understanding of the connection between electronic (optical) and atomistic (structural) behavior, will help describe the convergence of condensed matter and plasma physics-a critically missing link in basic scientific understanding-and will provide increased understanding of phase transitions and kinetics for EOS data development in support of stockpile stewardship.

In FY2006, the team published two sets of groundbreaking results in Physical Review Letters - the first-ever determination of critical lattice energy density required for solidplasma transition under ultrafast laser excitation and the first-ever measurement of the broadband dielectric function of warm dense gold, showing the role of intra- and interband transitions in the intermediate phase. These achievements were also reported in Physics Today and the American Physics Institute's Physics News Update. Also in FY2006, the team used its single-state data to make the first benchmark of Purgatorio calculations of electron collision time, direct current conductivity, and electron density and finished designing and constructing an ultrafast electron gun and a novel diagnostic for measuring the electron bunch length. In recognition for these achievements, this LDRD team received an LLNL Science and Technology Award for FY2006. 
Table 1. Patents resulting from LDRD-funded research as a percentage of all LLNL patents from 1999 to 2006.

\begin{tabular}{|l|c|c|c|c|c|c|c|c|}
\hline & 1999 & 2000 & 2001 & 2002 & 2003 & 2004 & 2005 & 2006 \\
\hline All LLNL patents & 84 & 93 & 89 & 97 & 71 & 95 & 93 & 63 \\
\hline LDRD patents & 45 & 35 & 42 & 27 & 29 & 55 & 51 & 29 \\
\hline $\begin{array}{l}\text { LDRD patents as } \\
\text { percentage of } \\
\text { total }\end{array}$ & $54 \%$ & $38 \%$ & $47 \%$ & $28 \%$ & $41 \%$ & $58 \%$ & $55 \%$ & $46 \%$ \\
\hline
\end{tabular}

\section{Patents}

Projects sponsored by LDRD consistently account for a large percentage of the patents issued for LLNL research, especially considering that beginning in FY2006 the LDRD Program funding represents $8 \%$ of the Laboratory's budget (i.e., $8 \%$ burdened, or no less than $6 \%$ in available funding). Before that, LDRD accounted for $6 \%$ of the total LLNL budget, with the exception of FY2000, when the LDRD Program received only $4 \%$ of the budget. Table 1 shows the number of patents resulting from LDRD-funded research since 1999.

The year for which a patent is listed is the year in which the patent was granted; LDRD investment in a technology is sometimes made several years before the technology is actually patented. Furthermore, although an LDRD-sponsored project makes essential contributions to such technologies, subsequent programmatic sponsorship also contributes to a technology's further development.

\section{Collaborations}

Collaborations are absolutely essential to the conduct of research and development in LDRD. By collaborating formally and informally with other national laboratories, academia, and industry, LDRD principal investigators (PIs) are able to access world-leading facilities and knowledge-both in the U.S. and abroad-and serve as active and prominent members of the broad scientific and technical community. This, in turn, allows LDRD PIs to leverage LLNL's achievements and capabilities. In addition, LDRD collaborations often result in outside scientists and engineers being recruited to LLNL.

In FY2006, the PIs of 56 LDRD projects (30\% of funded projects) engaged in a total of 74 formal collaborations. These institutions consist of the University of California ( $49 \%$ of total collaborations), other academic institutions (34\%), DOE sites (7\%), and other collaborators (e.g., other government agencies and industry, 10\%). These statistics do not include the numerous informal collaborations that PIs pursue in the course of their LDRD projects. 


\section{Awards}

- AAAS Fellow. Karl van Bibber was one of two Laboratory scientists selected in 2006 as fellows by the American Association for the Advancement of Science (AAAS). The fellowship was awarded in recognition for van Bibber's "distinguished contributions to the field of astrophysics and particle accelerator physics"-both fields in which he received LDRD support (including 91-DE-013, 94-DI-008, 97-SI001, and 00-SI-005). In addition, van Bibber served as deputy director of LLNL's Laboratory Science and Technology Office, which manages the Laboratory's LDRD Program, from 2002 to 2007.

- A\&BC Best Paper Award. A paper written by Laboratory scientist Darren Hillegonds won Analytical and Bioanalytical Chemistry's prestigious Best Paper Award for 2006. The paper described results achieved with LDRD support (04-ERI-009).

- APS Award for Excellence. Max Tabak was one of two LLNL plasma physicistsand four physicists in total-to receive the 2006 Award for Excellence in Plasma Physics Research from the American Physical Society. Tabak was cited "for developing the fast ignition inertial fusion concept and demonstrating key aspects of it in a series of experiments that have catalyzed the worldwide effort on the concept." Tabak's work on inertial fusion has been supported by LDRD at various critical junctures (99-FS-012, 02-ERD-041, and 04-ERD-054).

- APS Fellows. Of the five Laboratory scientists selected as American Physical Society fellows in 2006, LDRD has supported the pioneering work for which three were recognized-Vasily Bulatov (06-LW-028), Harry Radousky (02-ERI-006 and 06-LW-070), and Joe Wong (03-ERD-017).

- Edward Teller Fellows. Of the three recipients of 2006 Edward Teller Fellowships, two-Max Tabak and Vasily Bulatov-were awarded on the basis of track records in which LDRD support features prominently (for Tabak: 99-FS-012, 02-ERD-041, and 04-ERD-054 and for Bulatov: 06-LW-028).

- Global Life Science Innovation Competition. In UC San Francisco's 2006 Global Life Science Innovation Competition, LLNL scientist Darren Hillegonds received a second-place People's Choice Award for his poster presentation about his development of calcium-41 accelerator mass spectrometry-based assessment of bone health-work supported by LDRD (04-ERI-009).

- Gordon Bell Prize. A team led by LLNL physicist Fred Streitz received the 2006 Gordon Bell Prize in the "peak performance" category for a molecular-dynamics simulation performed on the BlueGene/L supercomputer. This work received LDRD support (02-ERD-033) in a critical early stage.

- LLNL 2006 Science \& Technology Award. A team whose work was substantially supported by LDRD received one of the two FY2006 Science and Technology Awards. (Although an internal award, this is based on a recipient's national and international recognition.) Andrew $\mathrm{Ng}$ was the leader of the team, whose groundbreaking results include the first-ever measurement of the broadband dielectric function of warm dense gold (04-ERD-108). 
- NIOSH Alice Hamilton Awards. A paper published by LLNL's Bioaerosol Mass Spectrometry (BAMS) team won honorable mention in the biological sciences category of the National Institute for Occupational Safety and Health's Alice Hamilton Award for 2006. Today's BAMS technology is the direct result of LDRD funding provided in the project's early crucial stages (99-ERD-013 and 02-ERD-002).

- PECASE Award. Former LLNL postdoctoral researcher Wendelin Wright received a Presidential Early Career Award for Scientists and Engineers. The work cited in the award-"research into the deformation and failure of metals and polymers under dynamic loading using high-speed and spatially resolved infrared measurements of temperature"-was partially funded by LDRD (04-ERD-005).

- R\&D 100 Awards. In 2006, Laboratory technologies won seven R\&D 100 Awards from R\&D Magazine. Of these, LDRD support contributed to five awards:

- An Ultrahigh-Resolution Gamma and Neutron Spectrometer (87-LW-007, 92-SR-046, and 01-LW-054).

- Babel (00-SI-002).

- Sapphire: Scientific Data Mining Software (99-ERI-010 and 03-ERD-031).

- Externally Dispersed Interferometry (98-ERD-054 and 04-ERD-067).

- High-Average-Power Wavelength Converter (97-ERD-138).

- Scientific American's "Most Important Science Story." Scientific American named the discovery of element 118 by an LLNL team and their Russian collaborators as one of the "most important science stories" of 2006. A key part of the Livermore team's work in this groundbreaking discovery was funded by LDRD (04-ERD-085).

- Top Minorities in Science Awards. LLNL scientist Hope Ishii was one of two recipients of Science Spectrum magazine's Top Minorities in Science "Trailblazer" Award for 2006. Work that Ishii conducted for an LDRD-funded Stardust project (03-ERI-007) was prominent in the achievements for which this award was presented. 
Laboratory Directed Research and Development

\section{Advanced Sensors and Instrumentation}




\title{
Ultrafast Transient-Recording Enhancements for Optical-Streak Cameras
}

\author{
Corey V. Bennett 04-ERD-025
}

\begin{abstract}
Several diagnostics at future large laser systems and advanced light sources such as the Linac Coherent Light Source will require hard $\mathrm{x}$-ray measurements with temporal resolution of about 1 ps or less and a high dynamic range, which is far beyond what can be achieved with existing instrumentation. We are investigating ultrafast optical-recorder technologies to develop a rugged, fiber-based temporal imaging system for opticalstreak cameras. This system will (1) improve system resolution and dynamic range by "stretching" an input signal in time, allowing ultrafast signal recording with slower, higherdynamic-range instruments and (2) convert light from 1550 to $775 \mathrm{~nm}$, allowing streak cameras to record signals from detectors using 1550-nm technology.
\end{abstract}

We expect to demonstrate single-shot operation of a robust temporal imaging system and streak camera recorder that produces a modulated optical carrier at $1550 \mathrm{~nm}$. This system will have less than 300-fs resolution and a high dynamic range (greater than 100 , and as high as 1000 at a slower magnified resolution) and be compatible with current and future hard x-ray detectors. In addition, we expect to develop a complete utrafast imaging system plan for implementation on large laser systems.

\section{Mission Relevance}

Our goal is to ensure delivery of the next-generation diagnostics needed for critical experiments at current and future large-laser systems, which is important for stockpile stewardship applications. These diagnostics will enable high-energy-density physics and inertial-confinement fusion experiments, including measuring reaction history, dynamic holhraum temperature, and dynamic opacity, as well as detecting sub-picosecond backscatter bursts.

\section{FY06 Accomplishments and Results}

In FY06, we designed and implemented a temporal imaging system utilizing unique fiberoptic dispersive delay lines in the input and time-lens pump arms of the system. Filters and amplifier stages were also included to improve the final signal-to-noise ratio. The time-lens utilizes sum-frequency mixing in a chirped, periodically poled lithium niobate waveguide because of the high-efficiency broadband mixing that can be obtained. The final output dispersion is created using a specially designed chirped fiber Bragg grating because of the extremely large dispersion with low loss that is obtainable. Ultrafast optical test patterns were propagated through the system and emerged time magnified by a factor of $M=-30.09$. We have demonstrated 1.8-ps resolution with nearly 1000:1 dynamic range, and have filed two records of invention based on our development of the imaging system. The success of this project has attracted federal agency support, and we will be developing an optical-frequency chirp diagnostic that is capable of making single-shot measurements of 100 ps pulses in a high-repetition-rate $(620 \mathrm{MHz})$ sequence of optical pulses. 


\title{
Publications
}

Chou, J., et al., 2006. Triggerable continuum source for single-shot ultra-fast applications. IEEE Lasers and Electro-Optics Society 2006 Ann. Mtg., Montreal, Canada, Oct. 29Nov. 2, 2006. UCRL-CONF-220029.

\section{An Integrated Laboratory for the Study of Interventional Device Dynamics}

\author{
Duncan J. Maitland 04-ERD-093
}

\begin{abstract}
The purpose of this project is to develop an integrated laboratory for investigating the physics and dynamics of endovascular interventional devices. This project will bring together four research components: novel endovascular devices, particle image velocimetry (PIV), computational fluid dynamic (CFD) models, and a core capability to generate physical and CFD models from actual human anatomies. We will apply the experimental and computational tools to a novel medical application: shape-memory polymer (SMP) foam for treating aneurysms.

If successful, the proposed research will improve the medical scientific community's understanding of endovascular interventional devices, enable novel device development, and assess impact on vascular fluid dynamics. We expect that this research at Lawrence Livermore has the potential to become an internationally accepted method to perform endovascular device research and development.
\end{abstract}

\section{Mission Relevance}

This project has direct relevance to Lawrence Livermore's mission in biotechnology to improve human health. In addition, the proposed system would enable direct analysis and subsequent design of fluidic systems used in devices for chemical and biological detection that are under development for the Laboratory's national and homeland security missions.

\section{FY06 Accomplishments and Results}

In FY06, we (1) demonstrated the SMP foam devices in vitro with flow and temperature measurements, and showed that we could successfully laser-deploy SMP foams to fill aneurysms without overheating the surrounding environment; (2) conducted nuclear magnetic resonance measurements of vascular flows and temperatures and demonstrated the potential for performing these measurements in vivo during device deployment; (3) successfully demonstrated an SMP stent and a hybrid stent-foam device, which was a deviation from the originally proposed bifurcation stent but has a potentially larger impact on future work; and (4) prepared publications on CFD results for predicting flow and temperature in aneurysms, material property studies, and significant results in nuclear magnetic resonance images of flow and temperature in the foam devices, foam device 
deployment, optical diffusing fibers, and PIV flow in aneurysms. In addition, we have filed several records of invention for our shape-memory polymer devices.

\title{
Proposed Work for FY07
}

In FY07, we plan to (1) construct an aneurysm temperature-measurement system, (2) conduct detailed studies of aneurysm wall temperatures as a function of laser delivery power to the SMP foam and blood flow rates using the cardiac flow system developed by the University of California at Berkeley-these temperature measurements will be used to assess the thermal effects of SMP heating on arterial tissue, and (3) perform a series of CFD simulations on anatomically correct aneurysms to assess the hemodynamic impact of the SMP-foam treatment technique.

\section{Publications}

Baer, G., et al., in press. "Shape-memory behavior of thermally stimulated polyurethane for medical applications," J. Appl. Polymer Sci. UCRL-JRNL-224906.

Buckley, P. R., et al., 2006. "Inductively heated shape memory polymer for the magnetic actuation of medical devices." IEEE Trans. Biomed. Eng. 53(10), 2075.

UCRL-JRNL-215657.

\section{Developing Radiography for Advanced Radiography Capability at Future Large Fusion-Class Lasers}

\author{
Hye-Sook Park 05-ERD-006
}

\begin{abstract}
We propose to develop bright, high-energy, high-resolution one- and two-dimensional (1D and 2D) radiography capabilities for high-energy-density (HED) experiments at future high-power laser facilities. These experiments require probing imploding targets made of bigger, thicker, and more dense materials than those used in the current experiments. We will develop backlighters with an energy of $>15 \mathrm{keV}$ and spatial resolution of $<10 \mu \mathrm{m}$ to properly diagnose the experimental conditions. We will study high-intensity laser interaction and radiation transport in a small, confined volumes and develop (1) volume-localized radiation targets that reduce source size and enhance brightness, (2) high-resolution 1D and 2D radiography techniques, and (3) suitable $x$-ray detectors for these high energies.
\end{abstract}

If successful, this project will lead to a petawatt radiography diagnostic for use on HED experiments at future fusion-class lasers. We will have a suite of hard $x$-ray sources, optics, and detectors that will be applicable to high-resolution, high-energy x-ray radiography. We will also significantly enhance our knowledge of high-intensity laser 
coupling efficiencies and electron transport for short-pulse lasers. This will contribute to the development of reliable simulation models for numerous applications such as fast ignition, proton heating, laboratory astrophysics, and high-temperature, soliddensity physics.

\section{Mission Relevance}

This project supports the Laboratory's national security mission by providing optimal radiography techniques for stockpile stewardship experiments, such as material strength experiments, equation-of-state experiments, HED experiments with mid- to high- $Z$ capsules, and double-shell ignition experiments.

\section{FY06 Accomplishments and Results}

In FY06, we (1) designed and fabricated gold (22 keV) and samarium (40 keV) microwire and microdot targets for 2D radiography sources, (2) tested their performance by radiography of test grid patterns, (3) measured K-alpha yields from these small-volume sources, (4) tested various detectors such as curved crystal spectrometers and imaging plates, and (5) published our results. We did not experiment with contact radiography as originally planned because the preferred method for fusion-class lasers is now considered to be point-projection radiography with microwire targets, thanks to its pointing flexibility and reduced debris. Our initial results show that the more efficient microwire targets may produce a sufficient signal for 2D radiography.

\section{Proposed Work for FY07}

We will continue to test the $>15-k e V, 2 \mathrm{D}$ radiography sources to determine the best options for 2D radiography. Our Titan experimental data show that the microwire targets may be bright enough to be used for 2D radiography. Specifically, we will (1) enhance $\mathrm{K}$-alpha yield efficiency in a small-volume target through better target design and fabrication, (2) measure the spatial resolution from the microwire target sources, (3) study high-energy backgrounds related to HED targets and estimate the signal-tonoise ratio, (4) study 2D requirements of the HED experiments for fusion-class lasers, (5) continue utilizing integrated simulations to optimize sources, and (6) continue studying options for hard x-ray detectors.

\section{Publications}

Park, H. S., et al., 2006. "High-energy K-alpha radiography using high-intensity, shortpulse lasers." Phys. Plasma 13, 056309. UCRL-JRNL-217367.

Theobalk, W. et al., 2006. "Hot surface ionic line emission and cold $\mathrm{K}$ inner shell emission from petawatt-laser-irradiated Cu foil targets." Phys. Plasma 13, 043102.

UCRL-JRNL-217895. 


\title{
Nanobarometers: An in situ Diagnostic for High-Pressure Experiments
}

\author{
James S. Stolken \\ 05-ERD-009
}

\begin{abstract}
The mechanistic understanding of high-pressure phenomena requires the capability to probe local material response at high spatial resolution using experiments with complex loading history. The development of an in situ nanoscaled pressure sensor would provide a means to assess the quality of simulations with direct measurements of local peak pressure. The diagnostic developed under this project will consist of nanoscale sensors that are in contact with the medium to be measured, can record the local peak pressure, and may be analyzed using a variety of microspectroscopy techniques. The result will be an in situ nanoscale sensing capability providing local peak pressure information at resolutions of less than $1 \mu \mathrm{m}$ and that has been calibrated over a pressure range of 30 to 300 kbar.
\end{abstract}

Key issues to be addressed will include calibration and sensitivity analysis of the nanosensors to both quasi-static and shock-loading conditions. Major goals of the proposed research are to quantify the extent of pressure-induced changes in the sensor material, determine their dependence on sensor size, and establish the sensitivity of pressure-induced structural changes to static loading.

\section{Mission Relevance}

High-pressure phenomena are central to experiments that support stockpile stewardship and other national security missions. Such experiments rely heavily on computational simulations for interpretation of local conditions such as temperature and pressure history. The development of an in situ nanoscale pressure sensor will provide a means for assessing the quality of these simulations through direct measurement of local peak pressure and comparison with simulation. The proposed nanosensors complement many existing Laboratory programs in multiscale modeling, material failure and fracture, and laser-driven shock physics.

\section{FY06 Accomplishments and Results}

In FY06, a key question regarding the existence and nature of the densification mechanism in silica nanoparticles has been explored using our nanobarometer. The issue was whether or not the densification phenomena observed in bulk silica occurred in nanoparticles. The permanent shift in the Raman spectra is clearly visible, consistent with the published results in bulk silica glass. Not unexpected is the dependence of the Raman shift upon the particle size, a key research question to be addressed by this project. Results obtained this year show, as suspected, a clear change in the size dependence for the smallest particles. The success of this year's experiments has led to a filing of a record of invention for the nanobarometer and pursuit of a preliminary patent. 


\title{
Remote Sensing of Alpha and Beta Particle Sources
}

\author{
Nerine J. Cherepy 05-ERD-032
}

\begin{abstract}
Our objective was to decrease the radiation dose a worker receives in assessment and cleanup, while obtaining a clear image of exactly where dispersed radiological contamination is located. To this end, we developed (1) a specialized ultraviolet (UV) viewer, (2) optimized scintillating coatings, and (3) a spectroscopic understanding of airglow and scintillator materials. Our UV viewer has two modes of detection: high and low surface activity. In the former, UV airglow due to energy deposition by alpha and beta particles from unshielded radiological materials is used to image and locate highactivity debris at a nuclear release. In the low-surface-activity mode, UV scintillation from a scintillating coating affixed to a contaminated surface is imaged to map very low-level surface activity and distribution.
\end{abstract}

We built a prototype viewer and demonstrated standoff imaging capability for alphaand beta-particle-emitting sources. This is a completely new detection methodology for radiological materials, allowing location and imaging at a safe working distance.

\section{Mission Relevance}

LLNL currently provides support to federal emergency responders that deal with nuclear emergencies, nuclear accidents, and nuclear terrorism. The technology we are developing would provide a useful tool for these applications in support of the Laboratory's homeland security mission. This technology also has potential uses in stockpile stewardship and monitoring of weapons-of-mass-destruction activities.

\section{FY06 Accomplishments and Results}

In FY06 we (1) optimized the "solar blind" (UV C-band, $<300 \mathrm{~nm}$ ) light yield of our scintillating coatings by synthesizing a range of UV-emitting scintillator particles and measuring their light yields and radioluminescence spectra; (2) completed two designs of portable UV viewers; (3) developed a multiple-variable spreadsheet model allowing us to calculate detection limits for alpha and beta sources outdoors under variable lighting conditions and with different UV viewers; (4) demonstrated detection under various conditions to benchmark the spreadsheet model; and (5) filed a patent for "garnet UV phosphor and scintillator materials preparation and use in radiation detection." The work done in this project has set the stage for continued development and eventual deployment of this technology. 


\title{
A Multiplexed Diagnostic Platform for Point-of-Care Pathogen Detection
}

\author{
James M. Birch \\ 05-ERD-049
}

\begin{abstract}
The goal of this project is to develop a practical, validated diagnostic tool with multiplexed nucleic-acid assays that can be used to simultaneously detect and identify multiple respiratory pathogens and to distinguish pathogens that cause common respiratory infections from those that are biothreat agents. We propose to (1) develop a multiplexed polymerase chain reaction (PCR) assay panel for simultaneous detection of multiple respiratory pathogens, (2) optimize assay performance in complex sample matrices, (3) validate the multiplexed PCR assays and diagnostic platform using clinical samples, and (4) demonstrate an autonomous, rapid bedside diagnostic device that can process and analyze a patient sample and post results in less than an hour.
\end{abstract}

This work will result in a prototype of a rapid, practical, and fully validated diagnostic tool with multiplexed nucleic-acid assays for pathogen detection in biowarfare or bioterrorism scenarios, for civilian preparedness, and for public health. This point-of-care diagnostic tool will find application in state and local public health laboratories and hospitals, clinics, and other health-related institutions. The rapid bedside diagnostic capability of this instrument has the potential for improving patient management.

\section{Mission Relevance}

A biologically based, point-of-care, pathogen-detection instrument has numerous potential biodefense applications for Lawrence Livermore's homeland security and national security missions and supports the Laboratory's mission in biotechnology to improve human health. It also enables applications that may support Centers for Disease Control missions in public health.

\section{FY06 Accomplishments and Results}

In FY06, we (1) discovered unique RNA signatures for five of the most commonly seen respiratory pathogens; (2) developed a bench-top assay using both negative patient samples spiked with these pathogens to determine sensitivity, as well as positive patient samples collected in collaboration with the University of California at Davis; and (3) began preparing our instrument for field testing at the UC Davis Medical Center.

\section{Proposed Work for FY07}

Because a larger respiratory panel would have greater impact on public health and biosecurity and gain greater acceptance in the medical community, we propose to use the same multiplexed assay techniques to expand our current panel to include nucleic-acid signatures that will discern (1) Streptococcus infections from Staphyloccus infections, (2) viral meningitis from bacterial meningitis, (3) methicillin-resistant Staphylococcus 
aureus, (4) whooping cough (Bordetella pertussis), (5) anthrax, (6) pneumonia (both Streptococcus pneumoniae and Mycoplasma pneumoniae), and (7) metapneumovirus. This larger panel would allow faster diagnosis and directed treatment for difficult diagnostic problems. Additionally, we will ready our instrument for field testing for the 2006-07 flu season.

\title{
Rapid Screening of Human Effluents with Single-Particle Mass Spectrometry for Early Detection of Respiratory Disease and Cancer
}

Matthias Frank 05-ERD-053

\begin{abstract}
This project will adapt single-cell bioaerosol mass spectrometry (BAMS) to real-time analysis of human effluents, such as exhaled aerosols, and screen those effluents for pathogens. The BAMS technique can analyze the biochemical composition of aerosol particles and single cells in real time and may be used to detect pathogens or cancerous cells. Research will begin by analyzing exhaled aerosol particles and droplets, then expand to include other human effluents, such as urine, for pathogens or cancerous cells.

If successful, the project will demonstrate that the BAMS technique can rapidly analyze human effluents and be used in the early detection of respiratory diseases and some cancers. This will advance biomedical diagnostics capabilities for respiratory diseases, analysis of biological aerosols, and the study of biochemical processes at the cellular level. It also will improve capabilities for emergency response to bioterrorist attacks. Furthermore, this project provides new avenues for strong collaborations with researchers at the University of California campuses of Davis and Berkeley.
\end{abstract}

\section{Mission Relevance}

If successful, this project will provide new capabilities for early detection of respiratory disease and cancer. Applications include biological weapons detection, population screening, and incident-response capabilities in support of Lawrence Livermore's national security and homeland security missions, as well as the Laboratory's mission in biotechnology for improving human health.

\section{FY06 Accomplishments and Results}

In FY06, we continued exploring characteristic mass signatures from pathogen surrogates and marker molecules measured by BAMS. By measuring mass spectral profiles from various lipids, we demonstrated that BAMS has the potential to distinguish aerosol particles originating in the lung (i.e., particles containing lung surfactant polar lipids) from particles originating in saliva (those containing nonpolar lipids). We also showed that mass spectral markers from RNA (simple influenza surrogate) and Mycobacterium smegmatis (tuberculosis surrogate) could be observed when these surrogates are embedded in lung 
surfactant (a model of the complex human matrix). We also initiated a collaboration with infectious disease clinicians from the UC Davis Medical Center to pursue deploying BAMS to a hospital.

\title{
Proposed Work for FY07
}

In FY07, we will translate our success in identifying mass spectral signatures of a tuberculosis surrogate to that of live virulent strains of Mycobacterium tuberculosis itself, as well as influenza. We also will extend our results to detection at a single-cell level. We then plan to demonstrate the feasibility of detecting respiratory pathogens in nasal swabs and sputum with BAMS and to measure biomarkers from well-characterized cancer cell lines provided by our collaborators at the UC Davis Cancer Center.

\section{Publications}

Tobias, H. J., et al., 2006. "Following the metabolic and morphological changes of individual Bacillus atrophaeus cells during the sporulation process using bioaerosol mass spectrometry." J. Microbiol. Meth. 67, 56. UCRL-CONF-221679.

\section{Probing Other Solar Systems with Current and Future Adaptive Optics}

Bruce A. Macintosh

05-ERD-055

\begin{abstract}
Over the past decade, Doppler techniques have allowed astronomers to infer the existence of more than a hundred planets orbiting nearby stars-discoveries of great scientific and public interest. The next step will be direct detection and characterization of extrasolar planets. Detection of a small number of such planets is barely within the reach of current adaptive optics (AO) systems. Development of a next-generation, dedicated planethunting, high-contrast "extreme" AO (ExAO) system is needed to probe the environments of other stars on scales comparable to that of our solar system. This project will use existing $\mathrm{AO}$ to probe possible planets in nearby solar systems and develop the precision optical technology needed for a Livermore-led future ExAO system for the Gemini Observatory.

This project will carry out a search for young extrasolar planets orbiting nearby stars, using advanced image-processing techniques to separate planets from background noise. Direct detection of a planet orbiting a nearby star would be a major scientific achievement, opening new windows into the formation and nature of solar systems. We also will develop advanced $\mathrm{AO}$ algorithms and sensors for very high-order precision $\mathrm{AO}$, allowing systems an order of magnitude more powerful than current technology to be controlled efficiently, optimally, and accurately. Technologies developed for this project will be key to a wide range of future AO work in remote sensing, laser beam control, biomedical applications, and the next-generation Gemini Planet Imager.
\end{abstract}




\section{Mission Relevance}

Development of ExAO techniques, such as wavefront characterization and correction, will provide Lawrence Livermore with a reservoir of skills and techniques for $A O$ that can be applied to large optical systems and space optics that will support national security missions in remote sensing for counterproliferation and nonproliferation missions. This project also supports the Laboratory's mission in breakthrough science and technology.

\section{FY06 Accomplishments and Results}

In FY06, we (1) discovered a candidate extrasolar planet near a young star by using our angular differential imaging technique to remove speckle noise from the AO images, (2) began follow-up observations of this extrasolar planet, (3) developed and published a new metrology technique for high-contrast $A O$ and a new high-efficiency wavefront sensor, (4) began the multiwavelength wave-optics modeling of AO systems, and (5) demonstrated subnanometer wavefront control at the University of California, Santa Cruz testbed, showing that the spatially filtered wavefront sensor reduces residual wavefront errors at midspatial frequencies by a factor of 10 . In addition, we published several conference papers and peer-reviewed journal articles on the results of our research.

\section{Proposed Work for FY07}

During FY07, we will (1) continue observations of candidate extrasolar planets discovered at the Keck Observatory, (2) carry out laser-guide-star observations of young brown dwarfs, (3) demonstrate the grating metrology technique, (4) test the performance of the ExAO wavefront-control algorithms on a high-order $\mathrm{AO}$ testbed, (5) complete an end-toend wave-optics model of an ExAO system to study diffraction and propagation effects in astronomical and other AO applications, and (6) publish the results of our Keck youngplanet surveys. Finally, a major goal will be the development of an AO control algorithm that predicts the motion of atmospheric turbulence driven by wind across the line of sight. This could potentially improve the performance of AO servo loops by a factor of two.

\section{Publications}

Evans, J., et al., 2006. "Demonstrating sub-nm closed loop MEMS flattening." Optic. Express 14(12), 5558. UCRL-JRNL-220228.

Evans, J., et al., 2006. "Effects of wavefront error on $10^{-7}$ contrast measurements." Optic. Lett. 31, 565. UCRL-JRNL-216023.

Evans, J., et al., 2006. "Extreme adaptive optics testbed: performance and characterization of a 1024-MEMS deformable mirror." Proc. SPIE 6113, 61130I. UCRL-ABS-216725.

Guenther, E., et al., 2006. "Low-mass companions to Hyades stars." Astron. Astophys. 442, 1031. UCRL-JRNL-218730.

Macintosh, B., et al., 2006. "MEMS-based extreme adaptive optics for planet detection." Proc. SPIE 6113, 611308, UCRL-ABS-217217. 
Marois, C., D. Phillion, and B. Macintosh, 2006. "Exoplanet detection with simultaneous spectral differential imaging: effects of out-of-pupil-plane optical aberrations." Proc. SPIE 6269, 62693M. UCRL-ABS-221155.

Marois, C., et al., 2006. "Accurate astrometry and photometry of saturated and coronagraphic point spread functions." Astophys. J. 647, 612. UCRL-JRNL-218773.

Marois, C., et al., 2006. "Angular differential imaging: a powerful high-contrast imaging technique." Astophys. J. 641, 556. UCRL-JRNL-216949.

Poyneer, L., and B. Macintosh, 2006. "Optimal Fourier control performance and speckle behavior in high-contrast imaging with adaptive optics." Optic. Express 14(17), 7499. UCRL-JRNL-219806.

Poyneer, L., et al., 2006. "Experimental demonstration of phase correction with a $32 \times 32$ MEMS mirror and spatially filtered wavefront sensor." Optic. Lett. 31, 293. UCRL-JRNL216885.

Poyneer, L., et al., 2006. "Wavefront control for the Gemini Panet Imager." Proc. SPIE 6272, 62721N. UCRL-ABS-220694.

\title{
RadTracker: Optical Imaging of High-Energy Radiation Tracks
}

Stephen P. Vernon 05-ERD-058

\begin{abstract}
The purpose of this project was to develop a solid-state, uncooled radiation detector for high-energy radiation particles using a novel optical imaging approach: Ionizing radiation generates electron-hole pairs, which in turn locally perturb the index of refraction, creating an optical-phase object track of the radiation. This can be sensed with an optical probe beam. Detector features would include (1) detection of $x$-rays, gammas, neutrons, and charged particles; (2) large operational energy bandwidth from $100 \mathrm{keV}$ to $>20 \mathrm{MeV}$; (3) high quantum efficiency of over $50 \%$ to enable longer standoff; (4) high energy resolution ( $>5 \%)$; (5) count rates in the megahertz range; and (6) directionality of radiation source without collimation (e.g., through electron-tracking Compton imaging for gammas).

Efficient radiation detection schemes for high-energy particles require large volumes of material to provide long particle-interaction lengths. The standard radiation detection approaches requiring charge transport and collection are often degraded in performance due to charge transport limitations over the long transport distances associated with these large volumes. The proposed approach was expected to resolve many of the critical technical questions surrounding optical imaging of high-energy radiation tracks for very large detector volumes without the performance degradation of existing approaches, thereby leading to greatly improved gamma imager performance.
\end{abstract}




\title{
Mission Relevance
}

Radiation detection is fundamental to several major DOE and LLNL missions. Novel, large-volume, high-performance radiation detectors can address large standoff radiation detection requirements for homeland security, as well as prompt diagnostic requirements for weapons physics experiments planned for large fusion-class lasers in support of stockpile stewardship.

\section{FY06 Accomplishments and Results}

In FY06, we developed model theories assuming resonant (exciton bleaching) and nonresonant (free-carrier) contributions to radiation-induced changes in the optical refractive index. In the model, the temporal dependence of index modulation was governed by diffusion of the radiation-induced charge. The model predicted extremely small optical scattering cross sections. To test this prediction, we conducted transient measurements using high-brightness pulsed radiation sources, along with quasi-continuous-wave experiments using amplitude-modulated sources. Consistent with the model predictions, both measurements produced noise-limited results. We concluded that this overall detection approach is not viable for single-transient, large-volume radiation detectors.

\section{Amplifier and Compressor Technology for Split-Beam, High-Energy Short Pulse Generation}

\section{Raymond J. Beach}

$$
\text { 05-ERD-062 }
$$

\begin{abstract}
A high-power, short-pulse laser would significantly enhance existing and planned research in high-energy-density science as well as the capabilities of inertial fusion facilities. To help develop the technology needed to realize such enhancements, this project will investigate the scientific and technical issues related to creating an integrated system for the injection, setup, compression, and monitoring of high-energy, split-beam, short-pulse generation from a single aperture of a neodymium-glass laser system. We aim to develop new amplifier and pulse compressor technologies that meet the stringent operating requirements and architectural constraints of large-scale amplification systems capable of producing kilojoule-class pulses.
\end{abstract}

If successful, we will develop new techniques for (1) multiple sub-aperture pulse generation and pulse-width control; (2) precision pointing and characterization of splitbeam pulses; (3) precision alignment of a folded, mixed-grating pulse compressor; (4) precision alignment and characterization of split-beam pulse compressors; and (5) multi-pulsing of large optical switches to block back-propagating beams on large-scale, kilojoule-class inertial fusion facilities. The ability to manipulate and combine multiple independent beams to achieve very-high-power, short-pulse operation represents a new paradigm in high-energy-density science and will enable multi-time-frame, K-alpha radiographic diagnostic capability. This research will produce important publications and intellectual property. 


\title{
Mission Relevance
}

This project furthers LLNL's missions in (1) stockpile stewardship by developing powerful new radiographic diagnostics; (2) energy security by furthering fast ignition, which would lead to potential fusion energy applications; and (3) breakthrough science and technology by enabling studies of matter under extreme conditions of temperature and pressure, with application in astrophysics, planetary physics, stellar interiors, and basic materials science.

\section{FY06 Accomplishments and Results}

Detailed requirements and specifications were generated for split-beam injection (SBI) into a neodymium-glass laser system, and the conceptual design for the SBI system was finalized. This effort focused on developing the simplest and most robust approach possible for SBI, which permits simultaneous operation of chirped-pulse beams and standard beams in the same quad. Our approach time-multiplexes the chirped pulse and standard beams together and then sends the compound pulse through a preamplifier system. The pulses are then time-demultiplexed and sent to independent beam lines. We have modeled both pre- and post-pulse leakage and determined the optimum optical isolator configurations necessary to meet target requirements for leakage.

\section{Proposed Work for FY07}

We will (1) demonstrate a prototype SBI system and use it to experimentally demonstrate the precision metrology required for beam pointing and centering and to determine the level of pre-pulse suppression necessary to prevent the chirped beams from impacting the utility of the standard nanosecond-duration beam lines; (2) conduct a full characterization of the SBI concept using prototype hardware; (3) carefully assess the level of isolation possible with our proposed optical isolator scheme; (4) investigate methods for the precision alignment of a folded, mixed-grating pulse compressor; and (5) investigate the use of coherent tiling and beam tiling.

\section{Publications}

Crane, J. K., et al., 2006. Status of the "ARC", a quad of high-intensity beam lines at the National Ignition Facility. International Conf. Ultrahigh Intensity Lasers 2006, Cassis, France, Sept. 25-29, 2006. UCRL-PROC-222453.

\section{Leading the Quantum Limit Revolution}

\author{
S. Darin Kinion \\ 05-ERD-073
}

\section{Abstract}

The goal of this project is to utilize microstrip superconducting quantum interference device (SQUID) amplifiers to revolutionize experiments, ranging from quantum coherence 
to particle astrophysics, that require improved signal-to-noise ratio. The primary deliverable will be a single electron transistor (SET) readout that is sensitive enough to enable single-spin detection in a solid-state system. We plan to develop robust packaging for the SQUID amplifier and then use the SQUID response to read out the signal from resonant-frequency SETs (rf-SETs). These experiments will be performed in collaboration with rf-SET experts at Yale University; the University of New South Wales, Australia; and the University of Maryland.

The most important result will be the demonstration of the rf-SET readout by a microstrip SQUID amplifier that is sensitive enough to detect a single spin in a solid-state system. This will be a breakthrough for quantum computations and quantum information applications. We expect to demonstrate the quantum limit for charge amplification and position measurement in nanomechanical resonators. Our results will be published in peer-reviewed journals, and university collaborations will attract top-echelon postdoctoral researchers to Lawrence Livermore.

\section{Mission Relevance}

This project will open the door to implementing quantum computing/quantum information secure communication architectures that support national and homeland security missions. This work also will support the Laboratory's mission in discovery-class science, such as the dark-matter axion experiment.

\section{FY06 Accomplishments and Results}

In FY06, we addressed several of the major issues in combining the microstrip SQUID amplifier with the rf-SET. Varactor diode tuning of the amplifier bandwidth was successfully demonstrated at millikelvin temperatures, allowing better frequency matching with the rf-SET tank circuit. A problem with the dynamic range of the amplifier was solved by implementing a scheme to cancel the carrier signal. Without the carrier, the full dynamic range of the SQUID amplifier can be utilized for the sidebands, which contain all of the information. The suppressed-carrier technique worked well and significantly improved the achieved charge sensitivity. Finally, the performance of the dilution refrigerator was dramatically improved, solving a major measurement problem.

\section{Proposed Work for FY07}

For FY07, the most significant hurdle remaining in the charge-sensitivity measurement is impedance mismatches between the SQUID and rf-SET, which leads to standing waves. As an alternative to isolators-which are lossy at millikelvin temperatures and too large to fit in a dilution refrigerator-we will explore using a pair of amplifiers in a balanced configuration, a common technique in conventional amplifier design. This should lead to a far better impedance match and eliminate standing waves. These amplifiers will then be used in the definitive charge-sensitivity measurements. Next, the amplifiers will be used in the first direct measurement of Josephson-junction $1 / f$ noise at millikelvin temperatures. Finally, we will assemble the first prototype high-frequency amplifier. 


\title{
Terascope: Terahertz Spectroscopic Imaging for Standoff Detection of High Explosives
}

\author{
Robert J. Deri \\ 05-ERD-076
}

\begin{abstract}
Standoff detection of the high explosive (HE) RDX-one of the most widely used ingredients in plastic explosives such as $\mathrm{C}-4$ and Semtex- $\mathrm{H}$-is difficult due to its low vapor pressure. This project is developing a standoff detection and identification approach for application to HE detection and identification. Our approach uses emerging spectroscopic and imaging technologies in the terahertz frequency regime. As part of the process of accumulating the scientific and technological knowledge needed to assemble an operational capability, we propose to first investigate the viability of the approach. Thus, we will execute a system-level analysis, a system simulation and, because we believe that detectable and decipherable signatures for common HE materials of arbitrary geometrical shapes and configurations are critical elements, we will execute an experimental program at the UC Santa Barbara free electron laser facility, a well-characterized source of tunable, monochromatic terahertz radiation.
\end{abstract}

The terahertz portion of the electromagnetic (EM) spectrum is rich with spectroscopic information about large molecules. Our primary goal is to assess the utility of terahertz spectral imaging for the detection of concealed, RDX-based explosives, by developing a systems concept and supporting, multispectral detection algorithms, and simulating the behavior of such a system in the presence of atmospheric absorption, obscurant losses, and system noise. Our strategy is to collect terahertz spectral data for materials of interest from both experiments and the literature, and combine it with systems analysis and simulation to assess the viability of the remote sensing capability. The results of this research will be published in peer-reviewed journals.

\section{Mission Relevance}

By developing a terahertz spectroscopy capability for the standoff detection and identification of covert HE devices, this research supports Laboratory missions in national security and homeland security.

\section{FY06 Accomplishments and Results}

In FY06, we (1) completed collection of terahertz data on common HE materials, (2) developed a systems concept and supporting algorithms for terahertz spectral imaging and, (3) simulated system performance. Results of these simulations show the proposed system can achieve standoff detection of bulk HE at distances of 30 to $50 \mathrm{~m}$, even when the explosive is concealed by a few layers of fabric. Simulated receiver operation characteristic curves, which show the probabilities of detection and false alarm, show excellent discrimination of C-4 against innocuous materials such as skin and lactose. 


\title{
Proposed Work for FY07
}

In FY07 we propose to verify experimentally our algorithms for spectral detection of RDX in the presence of atmospheric losses and concealants. We will first explore near-terahertz (800-900 GHz) imaging at the necessary standoff distances, then, if results are favorable, extend measurements to two lower frequency bands to obtain the required multispectral coverage in the subsequent year.

\section{Rapid Defense Against the Next-Generation Biothreat}

\author{
Raymond P. Mariella 05-ERD-084
}

\begin{abstract}
Bioengineered and emerging pathogens represent a significant threat to human health. The best defense against a rapidly expanding pandemic is to isolate the pathogen quickly from biological samples for analysis. The one persistent technology gap in the process of identifying and quantifying the presence of pathogenic agents has been sample handling and preparation that must precede any assay. The objective of this project is to replace burdensome, manual techniques with new automated technologies for sample handling and preparation. Specifically, we will use microfluidics with ultrasonic, electrophoretic, and dielelctrophoretic techniques to separate and purify viruses, the most transmissible and infectious agents, from biological and environmental samples.
\end{abstract}

We expect the new capabilities developed in this project to reduce the time required to identify a new pathogen (e.g., SARS) by up to an order of magnitude, in part by better matching methods for sample preparation with the needs of emerging assay technology. These capabilities will also be critical to developing ubiquitous, high-performance autonomous pathogen-sensing systems envisioned as sentinels that monitor for aerosoltransmitted pathogens by screening, for example, air filters, or handrails at international airports.

\section{Mission Relevance}

By making it possible to rapidly isolate, detect, and identify engineered and naturally emerging viruses, this project contributes to the nation's defense against bioterrorism, which is central to the Laboratory's homeland security mission. In addition, this project supports the Laboratory's mission in bioscience to improve human health.

\section{FY06 Accomplishments and Results}

In FY06 we (1) established a method to propagate the RNA virus MS2, harvest it, perform sample cleanup, and label the virus fluorescently for tracking and observation; (2) used millimeter-scale flow channels and both baker's yeast and MS2 to research particle transport via diffusion and via electrophoresis (EP), demonstrating that diffusion was too 
slow and that EP was rapid enough for our needs, with full transport achieved in under two minutes; (3) created and validated computer models to simulate these processes; (4) measured the zeta potential of both yeast and MS2 and demonstrated how our viral transport medium interferes with EP transport; and (5) conducted multiplex ligation probe amplification of viruses.

\title{
Proposed Work for FY07
}

In FY07, we will (1) model and design a combined diffusion-acoustic-EP separator for concentrating viruses from host material; (2) fabricate the first prototype of a multifield separation device; (3) test the performance of the multifield separation device, validate codes, and refine both; (4) solicit end-user evaluation and guidance for a lab-based beta unit (virologists at LLNL have volunteered to beta-test microfluidics that can separate released viruses from host cells and debris); (5) expand our sampling to 20-plex assays, including both RNA and DNA viruses; and (6) determine the limiting sensitivities and selectivities and research ways to maximize these, including varying target sequences and reaction conditions.

\section{Nonclassical Noise Reduction for Sensing Applications}

\author{
Richard P. Ratowsky 06-ERD-002
}

\begin{abstract}
In this project we will use theory, modeling, and simulation to examine the practical feasibility of exploiting the inherently quantum properties of the photon to reduce noise in sensing systems important to proliferation detection activities. We want to leverage recent developments in quantum optical noise reduction to exceed conventional noise limits and increase the sensitivity of remote sensing systems. We will calculate improvements in signal-to-noise ratios in specific scenarios versus system complexity and cost. To do this, we will develop a computational code and models and propose experiments to validate the conclusions.

If successful, this project will produce realistic estimates of performance versus hardware complexity to reduce noise using (1) squeezed light and (2) adaptive phase measurements. Ultimately, improved sensitivity with these technologies means detection with fewer photons. In practice, this translates to detection of proliferation signatures from greater distances. This work will help identify fruitful approaches for incorporating these technologies into future sensing systems.
\end{abstract}

\section{Mission Relevance}

By enhancing the sensitivity of photon-detection technologies used in countering the proliferation and use of weapons of mass destruction, this project supports LLNL's 
national security mission. Possible applications include remote sensing, free-space optical communications, and local optical sensing for pathogen and chemical detection.

\title{
FY06 Accomplishments and Results
}

In FY06 we reached a number of conclusions and formulated experiments designed to highlight the most promising directions for nonclassical detection. We determined that, because of the fragility of nonclassical light, quantum control must be applied at the receiving end of a remote sensing system, so that conventional coherent-state photons are propagated to the target and back and then subject to nonclassical processing in order to improve noise performance. We demonstrated how methods used in quantum communication can be applied to remote sensing based on phase measurements, and formulated and modeled an adaptive receiver using nonclassical processing. This project has laid the foundations for further experimentation directed at achieving received-state control.

\section{Biophysical Characterization of Pathogen Invasion}

\author{
Amy L. Hiddessen 06-ERD-013
}

\begin{abstract}
A fundamental understanding of pathogen infection and host response is needed to develop new treatments for infectious disease. To address this challenge, a comprehensive description of key processes is needed, including data on the spatiotemporal expression of molecular effectors and regulators of signaling cascades. Using a model pathogen system having well-characterized pathogen ligands and host receptors we will, for the first time, quantitatively characterize the virulent ligand-host cell-receptor interaction and subsequent signal transduction using novel signal delivery based on atomic force microscopy (AFM) that is combined with advanced optical methods.
\end{abstract}

Investigations of the complex phenomenon of pathogenesis will provide valuable knowledge about the molecular causes of infectious disease, as well as new insights into cell-regulatory machinery and signaling pathways in host cells. Successful completion of this project will provide the first detailed, quantitative picture of the initial process of infection with high spatiotemporal resolution. Moreover, this research will develop a novel single-cell platform for studying signal transduction in cellular systems and presymptomatic responses in the host cell. The results and tools generated in this work will be published and used to develop a new capability for a high-throughput, multiplexed pathogen-characterization system.

\section{Mission Relevance}

By combining synergistic experimental techniques to better understand pathogen-host interactions, this work will support Lawrence Livermore missions in biosecurity and basic bioscience research to improve public health. This project also supports LLNL 
missions to stay at the forefront of cutting-edge science in the areas of nanosciences and bionanotechnology.

\title{
FY06 Accomplishments and Results
}

In FY06, we (1) selected and acquired AFM instrumentation, (2) integrated the AFM with an existing confocal microscope, (3) initiated basic operational testing of the combined AFM-confocal microscope, and (4) submitted a record of invention based on our signal delivery for single-cell characterization.

\section{Proposed Work for FY07}

In FY07, we will (1) continue basic operational testing of the AFM-confocal instrument, (2) develop protocols for functionalizing AFM cantilevers with biomolecules, (3) optimize fluorescent protein labeling of actin in live epithelial cell lines and design custom reagents and protocols for labeling host-cell receptors, (4) conduct preliminary tests of AFM-based ligand delivery to live cells on the microscope stage, and (5) work with vendors to custom design a plan for incorporating advanced optics into the AFM-confocal instrument in preparation for visualizing and manipulating structures inside host cells.

\section{Publications}

Hiddessen, A. L., N. Shen, and T. A. Sulchek, 2006. Biophysical characterization of pathogen invasion and host cell response. UCRL-ABS-223863.

\section{Thermal-Fluidic System for Manipulating Biomolecules and Viruses}

\author{
Kevin D. Ness \\ 06-ERD-040
}

\begin{abstract}
We propose to develop a reconfigurable fluidic system that will simultaneously separate, concentrate, and purify biomolecules and viruses. Many pathogen detection, mitigation, and protection applications require manipulation of biomolecules or viruses to accurately quantify the presence of a particular substance or synthesize and investigate the function of a molecule. We will achieve this manipulation in a single system by utilizing a novel microfluidic technology, temperature gradient focusing (TGF), an equilibrium gradient version of capillary electrophoresis that allows for the stationary fractionation and concentration of target analytes based on bulk electrophoretic mobility.

Starting from the manually controlled proof-of-principle TGF system, we will develop a more robust system to concentrate and separate different viral strains. The ultimate purpose of this system is to facilitate viral characterization and identification. Expected results include a demonstrated improvement of the TGF separation resolution using a variety of TGF chip designs for fluorescent dyes, e-tags, proteins and viruses. Successful
\end{abstract}


demonstration of these capabilities will also establish the system's utility for other biological applications.

\title{
Mission Relevance
}

The project will contribute to the Laboratory's national security and homeland security missions in biodefense by demonstrating the ability to concentrate and separate viruses for fast identification and detection, and enhancing the production speed and efficiency of synthetic reagents for detection of threat toxins, bacteria, and viruses. Both applications support efforts that enhance the rapid detection of engineered or naturally emerging viruses, bacteria, or other toxins.

\section{FY06 Accomplishments and Results}

After a midyear start, we developed the experimental capability to determine input parameters for models of protein and virus capture and purification by TGF, and to benchmark predictions made with those models. To do so, we constructed a reconfigurable automated TGF workstation and quantified the reproducibility of subsystem components (e.g., pumps, valves, and temperature controllers) to identify equipment modifications necessary to improve the overall TGF system stability, reproducibility, and accuracy.

\section{Proposed Work for FY07}

In FY07, we will determine conditions for capturing and purifying viruses and proteins using TGF. We will define the initial experimental conditions such as flow rates, concentrations, buffer composition, temperature gradients, and electric fields using simple models specific to a given virus or protein sample. We will also perform preliminary experiments of characterized virus and protein mixtures to populate a data matrix for viruses that specifies electrophoretic mobility versus viral family or strain and a data matrix for proteins specifying molecular weight versus isoelectric point. These matrices will then be utilized as a reference to optimize the purification and separation of viruses and proteins from a "clean" sample.

\section{Viral Identification and Characterization Initiative (VICI)}

Billy W. Colston

06-ERD-064

\begin{abstract}
Humans are exposed daily to millions of viruses which are poorly understood. Identifying viruses is challenging, since many are impossible to culture and can evolve rapidly. This problem is compounded by our near total lack of knowledge about the "viral background"-the number and types of nonvirulent viruses normally found in our environment. Overcoming this barrier would enable huge leaps in our understanding of emerging diseases. We intend to use an approach similar to that of the Human Genome
\end{abstract}


Project and create a translational measurement capability that will allow rapid, highthroughput viral screening. Instead of trying to selectively isolate a virus from a potentially complex media, we propose to analyze every virus in a representative sample.

If successful, this project will produce technology for rapidly obtaining a complete viral genomic profile from almost any type of sample (e.g., urine, blood, and saliva). Like the Human Genome Project, this capability will have an almost inestimable impact on the nation's science. By tackling the huge technological hurdles associated with this challenge and beginning viral prospecting to better understand respiratory infections, we will create a vital new competency for the nation. This work will lead to a small set of high-profile journal articles focusing initially on human respiratory diseases.

\section{Mission Relevance}

As the recent SARS outbreak demonstrates, the identification of previously unknown or emerging viral pathogens can take months after a pandemic begins, despite Herculean efforts using existing technology. This work supports LLNL's national and homeland security missions by helping fill a critical gap in our nation's defense by creating the ability to rapidly and dynamically respond to such new biological threats, whether naturally arising or deliberately engineered.

\section{FY06 Accomplishments and Results}

After a midyear start, we focused on developing bench-top PCR assays for target viral DNA identification, a microfluidics platform for droplet generation, and on-chip PCR. In our bioinformatics effort we have computationally explored universal primer set space by generating a series of short ( $7-15$ base pairs) primers. The computational primer pairs were then tested in single-plex reactions under various reaction conditions. We found that we were able to generate predicted amplicons successfully with a large number of our primer pairs. We also have designed, developed, and tested a microfluidic chip with the ability to create sub-nanoliter aqueous droplets in a continuous oil stream, and have filed several records of invention related to viral identification and characterization.

\section{Proposed Work for FY07}

The first thrust will be to fully develop bench-top multiplexed assays and validate our ability to identify known and unknown viruses from banding patterns. This effort requires extensive experimental testing that is carefully guided by bioinformatics. The logical process starts with further optimization of PCR conditions for DNA amplification using short primers. Our second thrust will be to continue the development of the microfluidic platform. Our first major milestone will be to demonstrate successful PCR of DNA captured in a stream of microdroplets. This system will be fully automated to facilitate precise and repeated operation of the pumps, valves, heaters and detection systems. 


\title{
Publications
}

Beer, R., 2006. Microfluidics platform for viral detection. SPIE Annual Mtg., San Diego, CA, Aug. 13-17, 2006. UCRL-ABS-220620.

Hiddessen, A., 2006. Viral detection with short primer amplification. SPIE Annual Mtg., San Diego, CA, Aug. 13-17, 2006. UCRL-ABS-220445.

\section{Real-Time, Ellipsometry-Based Transmission Ultrasound Imaging}

Jeffrey S. Kallman

06-LW-093

\begin{abstract}
Our objective is to fill the need for fast, inexpensive two- and three-dimensional (2D and 3D) transmission ultrasonic imaging for use in nondestructive evaluation and medicine. The sensor resulting from this work could speed ultrasonic data acquisition by orders of magnitude and provide real-time inspection and medical imaging. Our plan is to build a large-area-imaging ultrasound sensor and examine its sensitivity, frequency response, resolution, and noise characteristics. The sensor would use ultrasound to modulate a beam of light in an ellipsometer, which can be recorded by a camera and computer.

We expect to produce a sensor capable of imaging an ultrasonic pressure field in real time. Considering that transmission ultrasound images are currently acquired using mechanically scanned point sensors, the new ellipsometry-based sensor will speed up industrial inspection of composite panels by three orders of magnitude. Transmission ultrasound is not in wide use as a medical imaging technology because of the time it takes to acquire data. This project will enable new generations of 3D breast-cancer imaging tools that require no ionizing radiation and no discomfort. If the frequency response is high enough, this technology may also enable a different set of laser-target characterization tools. We expect technical publications, patents, and licenses will result from this project.
\end{abstract}

\section{Mission Relevance}

The capability developed in this project will support the Laboratory's stockpile stewardship mission by providing new technology in nondestructive imaging. These technologies can also be used in medical imaging for breast-cancer detection and rapid diagnostics for orthopedics and foreign objects. This will support the Laboratory's biotechnology mission to improve human health. 


\section{FY06 Accomplishments and Results}

In FY06, we explored the coating of ambient-dried, low-density aerogel thin films. These were applied to optical substrates to form small sensors for imaging acoustic pressure fields over their entire surfaces in real time. However, there were difficulties in producing thin films with the properties needed to produce an imaging system. We developed an imaging test stand for evaluating the sensors, and determined that film formation techniques need to be refined. 
Laboratory Directed Research and Development

\section{Bjological Sciences}




\title{
Microbial Pathways
}

\author{
Harry R. Beller \\ 03-ERD-062
}

\begin{abstract}
This project makes use of emerging genome-enabled techniques-such as comparative genomics and whole-genome transcriptional studies with microarrays-to explore novel, bacterial metabolic pathways and bacteria-mineral interactions that can mediate the behavior of uranium and other environmentally relevant elements in the subsurface. This is particularly pertinent to a remediation process that is of great interest to DOE: the in situ, reductive immobilization of metals, including radionuclides. This project utilizes LLNL's existing capabilities in genomics, microbiology, molecular biology, biochemistry, geochemistry, and computational modeling in a significantly new research direction for bioscience.
\end{abstract}

Expected results include (1) publications in high-visibility journals regarding the microbially mediated transformations of uranium in anaerobic soil and aquifer environments and the genetic and biochemical basis of nitrate-dependent metal oxidation, (2) the finished and well-annotated whole genome of the bacterium Thiobacillus denitrificans for use by the scientific community interested in anaerobic metal oxidation, (3) a genetic system in T. dentrificans that will act as a highly valuable molecular tool for knocking out (disabling) genes to better understand their role in functions of interest (application is not limited to anaerobic metal oxidation), and (4) a deeper understanding of relationships between nitrate-dependent uranium(IV) and iron(II) oxidation.

\section{Mission Relevance}

This project will further biogeochemistry that supports LLNL's mission in environmental management and restoration. Microbial processes active in the near-surface environment are important considerations in national security, especially issues concerning radionuclide and toxic metal contamination of groundwater. The proposed project is anticipated to encompass collaborations both within the Laboratory and with the DOE Joint Genome Institute.

\section{FY06 Accomplishments and Results}

Accomplishments in FY06 include (1) demonstrating iron(II) enhancement of nitrogenoxide-dependent uranium(IV) oxidation by $T$. dentrificans, (2) completion of a genetic system for $T$. dentrificans and demonstration of its effectiveness, (3) use of reversetranscription polymerase chain reaction analysis to confirm microarray results, and (4) preparation of a manuscript on whole-genome microarray results. This project accomplished its major goals: We established $T$. dentrificans as the first autotrophic bacterium capable of anaerobic uranium(IV) oxidation, generated extensive microarray data on whole-genome gene expression under uranium(IV)- and iron-oxidizing conditions, provided manual annotation of the $T$. dentrificans genome, provided a highly valuable genetic system to perform gene knockout experiments in $T$. dentrificans, and produced three high-visibility publications and numerous talks at high-profile conferences. 


\section{Publications}

Beller, H. R., 2005. "Anaerobic, nitrate-dependent oxidation of U(IV) oxide minerals by the chemolithoautotrophic bacterium Thiobacillus denitrificans." Appl. Env. Microbiol. 71, 2170-2174. UCRL-JRNL-204909.

Beller, H. R., 2005. Anaerobic, nitrate-dependent oxidation of uraninite by the chemolithoautotrophic bacterium Thiobacillus denitrificans. Amer. Society for Microbiology, 105th General Mtg., Atlanta, GA, June 8, 2005. UCRL-ABS-208811.

Beller, H. R., A. Chakicherla, and T. C. Legler, 2005. Anaerobic, nitrate-dependent oxidation of U(IV) by the obligate chemolithoautotroph Thiobacillus denitrificans: cell suspension and whole-genome transcriptional studies. Joint Intl. Symp. Subsurface Microbiology (ISSM 2005) and Environmental Biogeochemistry (ISEB XVII), Jackson Hole, WY, Aug. 14, 2005. UCRL-ABS-213008.

Beller, H. R., et al., 2005. Anaerobic, nitrate-dependent oxidation of uraninite by the chemolithoautotroph Thiobacillus denitrificans: cell suspension and whole-genome transcriptional studies. American Geophysical Union Fall Mtg., San Francisco, CA, Dec. 5-9, 2005. UCRL-ABS-216212.

Beller, H. R., et al., 2005. The genome sequence of the chemolithoautotrophic betaproteobacterium Thiobacillus denitrificans: a small yet metabolically versatile genome. Amer. Society for Microbiology 105th General Mtg., Atlanta, GA, June 8, 2005.

UCRL-ABS-208813.

Beller, H. R., et al., 2005. "The genome sequence of the obligately chemolithoautotrophic, facultatively anaerobic bacterium Thiobacillus denitrificans." J. Bacteriol. 188, 1473-488. UCRL-JRNL-215002.

Beller, H. R., et al., 2005. Transcriptional analysis of chemolithoautotrophic $S$ oxidation by Thiobacillus denitrificans under aerobic vs. denitrifying conditions using whole-genome oligonucleotide microarrays. Amer. Society for Microbiology 105th General Mtg., Atlanta, GA, June 8, 2005. UCRL-ABS-208812.

Beller, H. R., et al., 2006. "Whole-genome transcriptional analysis of chemolithoautotrophic thiosulfate oxidation by Thiobacillus denitrificans under aerobic versus denitrifying conditions." J. Bacteriol. 188, 7005-7015. UCRL-JRNL-220798.

\section{Force Spectroscopy to Study Multivalent Binding in Protein-Antibody Interactions}

Todd A. Sulchek

03-ERI-009

\section{Abstract}

This project is motivated by the need to increase the time over which cancer therapeutics using multiple antibodies to attach to cancer cells remain in the bound state. The two goals of this project are to determine the dynamic strength of multiple bonds and to 
observe the limits of cellular response to multivalent protein binding. To accomplish this, we will use atomic-force microscopy to measure the binding strength between multiple Mucin1-antibody bonds and also to measure the effect of multivalent binding of fibronectin protein to fibroblast cell integrin receptors. Our approach is to link fibronectin to the tip of an atomic-force microscope cantilever, deliver a controlled number of molecules to the cell surface, and observe the labeled actin response with a confocal microscope.

By measuring the actin response to fibronectin binding, we expect to gain an understanding of the lower limits of cellular response to external signals. The primary question we expect to answer is whether the cell requires a threshold amount of fibronectin to trigger a response.

\section{Mission Relevance}

The capability to measure antibody-protein interactions, combined with expertise in suspended bilayers, will enable other biological applications such as characterizing cellpathogen interactions, that are of interest to Lawrence Livermore's homeland security mission. In addition, research on understanding drug-cell and cell-cell interactions supports the Laboratory's mission in bioscience to improve human health.

\section{FY06 Accomplishments and Results}

In FY06, we completed our studies of multivalent binding at synthetic surfaces and moved towards measuring interactions with living cells. We assembled a unique instrument to quantify the actin cytoskeletal response in a fibroblast cell to multivalent fibronectin binding. The instrument consisted of a high-sensitivity, confocal fluorescent microscope combined with a high-resolution, atomic-force microscope. It allowed us to position the cantilever probe of the atomic force microscope over a fluorescently labeled cell and touch the probe to the cell at a precise location and for a precise amount of time. During the year, we used the instrument in recording time-dependent responses to the contact of a functionalized probe with the surface of a target cell. Overall, this project was extremely successful, and resulted in several invited talks and publications in peer-reviewed journals.

\section{Publications}

Sulchek T. A., R. W. Friddle, and A. Noy, 2005. "Strength of multiple parallel biological bonds." Biophys. J. 90, 4686. UCRL-JRNL-217747.

Sulchek, T. A., et al., 2005. "Dynamic force spectroscopy of parallel individual Mucin1antibody bonds." Proc. Natl. Acad. Sci. Unit. States Am. 102, 16638. UCRL-JRNL-214131. 


\title{
Intracellular Chemical Measurements: A Generalized Approach with High Spatial Resolution Using Functionalized Nanoparticles
}

Ted A. Laurence

03-ERI-010

\begin{abstract}
The objective for this research is to develop methods to measure concentrations in chemical microenvironments in cells and tissues using recently developed, functionalized metal nanoparticles (50-100 nm diameter). Surface-enhanced Raman spectroscopy (SERS) allows sensitive detection of changes in the state of chemical groups attached to single nanoparticles. We will test a nanoscale $\mathrm{pH}$ meter in a cell-free medium to measure the $\mathrm{pH}$ of the solution immediately surrounding the nanoparticles, then test the nanoscale $\mathrm{pH}$ meter inside cells to determine optimal conditions and methods for these measurements. The nanoscale $\mathrm{pH}$ meter will be used to image the local $\mathrm{pH}$ inside and outside cells in tumor tissues.
\end{abstract}

This novel measurement technique promises to provide rigorous quantitation of cellular concentrations, opening new windows to cell characteristics and behavior. In tumor cells, it will be possible to monitor the effects of the local pH gradient on uptake of various therapeutic agents. (Other methods suffer from poor spatial resolution or high cell toxicity.) A decrease in $\mathrm{pH}$ will serve as an indicator of apoptosis; correlation with stresses or other signals will help identify events, causes, and phenomenology of apoptosis. Success with the proposed experiments will demonstrate the ability of SERS nanoparticles to measure local pH inside cells. This will encourage development of the SERS technology to measure other chemical concentrations inside microenvironments.

\section{Mission Relevance}

Measuring chemical gradients (variations in concentration) across membranes is important for understanding basic biological processes and is relevant to developing disease treatments. This research supports development of a novel method for measuring chemical concentrations. Such probes of the intracellular environment represent a key enabling capability for the DOE GtL:Genomics Program and biosecurity applications. In addition, the new measurement technique supports LLNL applications related to bioscience and technology to improve human health, as well as breakthroughs in fundamental bioscience.

\section{FY06 Accomplishments and Results}

In FY06, we developed a rapid imaging technique for monitoring $\mathrm{pH}$ in heterogeneous environments, including living cells. We also developed a rapid assay for characterizing surface-enhanced Raman scattering signals from metallic nanoparticles. Using this assay, we demonstrated that hollow gold nanoshells developed by collaborators have more consistent scattering properties than previously used solid gold and silver nanoparticles. This work resulted in a patent application. Overall, this project's successes have paved the way for further work to address additional related issues. 


\title{
Publications
}

Laurence, T. A., S. Fore, and T. Huser, 2006. "Fast, flexible algorithm for calculating photon correlations." Optic. Lett. 31, 829-831. UCRL-JRNL-216336.

\section{Using Femtosecond Laser Subcellular Surgery as a Tool to Study Cell Biology}

\author{
Nan Shen \\ 03-ERI-012
}

\begin{abstract}
Research on cellular function and regulation would be greatly advanced by new instrumentation using methods to alter cellular processes with spatial discrimination on the nanometer scale. We apply a novel technique for targeting subcellular organelles and other biologically important regions in living cells using femtosecond laser pulses. By tightly focusing the laser pulses inside a cell, we achieve disruption of cellular material through nonlinear optical processes. This technique enables noninvasive manipulation of physical structures of a cell with submicrometer resolution. Using this method, we study the role mitochondria play in cell proliferation and apoptosis by selectively perturbing mitochondria in living cells.

Our experimental apparatus will allow us to study cell behavior in real time to address questions such as how cells behave in the long term after laser surgery and whether disruptions of different cellular structures result in different cell responses. We hope to induce apoptosis by disrupting mitochondria in a cell to study the process of cell death and to see whether the number of functional mitochondria is critical to the survival of a cell and whether mitochondria are capable of repairing structural damage. Another focus of the project is on how cell cytoskeleton reorganizes following structural perturbation. We want to follow the dynamics of cytoskeletal remodeling after laser disruption of microtubules and actin filaments.
\end{abstract}

\section{Mission Relevance}

We plan to demonstrate new LLNL capability utilizing laboratory expertise in both laser and life sciences. The resulting data will support DOE missions in understanding cellular mechanisms. Furthermore, this understanding will ultimately prove important in helping to determine complete mechanisms for pathogenicity, in support of homeland security applications.

\section{FY06 Accomplishments and Results}

The instrument was designed to achieve simultaneous manipulation of cell structures and real-time cellular response using optical microscopy. We reconfigured our system to include total internal reflection microscopy for high-resolution imaging down to the singlemolecule level, examined DNA damage after mitochondria disruption, and investigated 
cytoskeleton remodeling after creating microtubule lesions by monitoring the motion of stress-bearing fibers as a function of laser energy. This project established femtosecond laser surgery as a noninvasive method for structural disruption within live cells. The method was used to study mitochondria function in combination with biochemical methods, and the results obtained can be applied towards future research on pathogencell interaction.

\title{
Publications
}

Shen, N., et al., 2006. Studying cellular function by targeted disruption of structures inside living cells with a femtosecond laser scissor. 2006 Biophysical Soc. Mtg., Feb. 18-22, 2006, Salt Lake City, UT. UCRL-ABS-218940.

\section{Multiprobe Investigation of Proteomic Structure of Pathogens}

\author{
Alexander J. Malkin 04-ERD-002
}

\begin{abstract}
This project will investigate the proteomic structure, architecture, and function of bacterial spores through a combination of high-resolution in vitro atomic force microscopy (AFM) and AFM-based immunolabeling with threat-specific antibodies. This experimental approach will allow us to visualize, immunolocalize, and identify both surface and internal protein components of bacterial spores, model their complex architecture, and establish the relationships between pathogen structure and function. A more comprehensive understanding of the architecture and properties of bacterial spores would contribute significantly to a general understanding of their life cycle and may lead to advances in diagnostic and immunological aspects of anthrax biodefense.

By elucidating the structurally related properties and function of bacterial spores and modeling their architecture, this work will provide a foundation for more efficacious diagnostic, chemotherapeutic, and immunological countermeasures for emerging diseases and biodefense. This work will reveal the architecture, structure, and molecularscale mechanisms of the therapeutic germination process of spores of the engineered bacterium Clostridium novyi-NT and will add new dimensions and insights to a pioneering bacteriolytic therapy for cancer treatment. This research will also produce published papers and conference presentations.
\end{abstract}

\section{Mission Relevance}

By developing AFM for the evaluation of pathogenic morphological signatures and structural attributes, this project supports the Laboratory's national security and homeland security missions by developing techniques for identifying and characterizing pathogens that could be used by bioterrorists. In addition, this work will provide fundamental information for modeling pathogen architectures for use in structural biology, in support of the Laboratory's mission in biosciences to improve human health. 


\section{FY06 Accomplishments and Results}

In FY06 we performed the first-ever in vitro visualization of the complete architecture of the spore coat of $C$. novyi-NT spores; in vitro real-time, high-resolution analyses of germination mechanisms of Bacillus and Clostridium single spores; and in vitro visualization of the high-resolution native peptidoglycan structure of the cell wall of Bacillus cells. We also demonstrated formulation-dependent assembly of the outer spore coat of Bacillus spores, developed experimental approaches for AFM-based immunolabeling, produced antibodies with immunochemical specificity for protein components of the spore coat for Bacillus and Clostridium species, and helped establish a Biosafety Level 2 AFM facility for studies of anthrax vaccine strains and other threat pathogen surrogates.

\section{Proposed Work for FY07}

In FY07 we will (1) complete the molecular structural characterization of the Bacillus anthracis exosporium and spore coat as a function of processing and formulation procedures and exposure to priority decontamination agents; (2) complete proteomic mapping studies of the surface structures of $B$. anthracis and $C$. novyi-NT dormant spores, germinating spores, and vegetative cells; (3) complete evaluation of the impact of chemotherapeutic drugs and inhibitors on the germination process of $C$. novyi-NT spores; and (4) complete characterization and mapping of $B$. anthracis spore coat and exosporium surface charge distribution as a function of species, preparation, and environmental conditions using chemically functionalized nanogold probes.

\section{Publications}

Malkin, A. J., et al., 2005. "Unraveling the architecture and structural dynamics of pathogens by high-resolution in vitro atomic force microscopy." Proc. 3rd Latin American Symposium on Scanning Probe Microscopy. UCRL-PROC-211437.

Malkin, A. J., et al., 2006. Unraveling the structure-function relationships of microbial systems by high-resolution in vitro atomic force microscopy. Seeing at Nanoscale IV, Philadelphia, PA, July 17-20, 2006. UCRL-CONF-220631.

Malkin, A. J., et al., in press. "Virus architecture probed by atomic force microscopy," Structure-based study of viral replication. World Scientific Publishing Co., Inc., Hackensack, NJ. UCRL-BOOK-219826.

Plomp, M., et al., 2006. Probing the structure-function relationships of microbial systems. 2nd American Society for Microbiology-IEEE Engineering in Medicine and Biology Society Conf. Bio, Micro, and Nanosystems, San Francisco, CA, Jan. 15-18, 2006.

UCRL-PROC-216841. 


\title{
Developing New Tools for in vivo Generation and Screening of Cyclic Peptide Libraries
}

Julio A. Camarero

04-ERD-040

\begin{abstract}
The objective of this project is to develop a new combinatorial approach for the biosynthesis and screening of small, drug-like toxin inhibitors inside living cells. Initially, this novel approach will be used for finding inhibitors against the anthrax lethal factor (LF) bacterial toxins from Bacillus anthracis as proof of principle. This combinatorial technique combines the biosynthesis and screening of a library in the same step using a living cell as a small microchemical factory. This research will accelerate the process of discovering new ligands for any molecular target.
\end{abstract}

The success of this project would significantly impact biosecurity and drug discovery. It will introduce a new and generic technology that combines chemistry and biology for fast and efficient identification of high-affinity ligands for botulinum and anthrax LF bacterial toxins alike. These ligands could be used as powerful antidotes against the toxins and as recognition elements in biosensors attached to appropriate platforms. Furthermore, this new method can be easily generalized for finding small, drug-like effectors for any protein-protein interaction, which will have a tremendous impact in pharmaceuticals and proteomics.

\section{Mission Relevance}

By identifying ligands that have high affinity for botulinum and LF bacterial toxins, this project could lead to developing antidotes for biothreats and new biosensors to detect bioterrorist attacks. The project supports the Laboratory's national security and homeland security missions by furthering LLNL efforts to counter bioterrorism.

\section{FY06 Accomplishments and Results}

In FY06 we (1) finished development of a method for the in vivo biosynthesis and screening of libraries of circular peptides based on the cyclotide scaffold for the construction of large biological libraries in vivo and (2) developed a fluorogenic assay for the in vivo screening of cyclotide-based libraries against the anthrax LF. Thus this project achieved its major objectives, and has resulted in a record of invention for synthesis of cyclic peptides.

\section{Publications}

Camarero, J. A., and R. H. Kimura, 2006. "A cell-based approach for biosynthesis/ screening of cyclic peptide libraries against bacterial toxins." Protein Sci. 15, 273. UCRL-ABS-222780. 
Camarero, J. A., and R. H. Kimura, 2006. "Expressed protein ligation: A new tool for the biosynthesis of cyclic polypeptides." Protein Pept. Lett. 12, 789. UCRL-JRNL-208023.

Camarero, J. A., R. H. Kimura, and A. T. Tran, 2006. "Biosynthesis of the cyclotide kalata B1 using a protein splicing unit." Angew. Chem. Int. Ed. 45, 973. UCRL-JRNL-214692.

Camarero, J. A., R. H. Kimura, and K. Krishnan, 2006. "Biosynthesis of the cyclotide kalata B1 using protein splicing tools." Biopolymers 80, 537. UCRL-CONF-214132.

Camarero, J. A., R. H. Kimura, and A. T. Tran, 2006. "A cell-based approach for biosynthesis/screening of cyclic peptide libraries against bacterial toxins." J. Pept. Sci. 12, 89. UCRL-ABS-222776.

\title{
A Coupled Computational and Experimental Approach to Determine Functions of Deeply Conserved "Anonymous" Human Genes
}

\author{
Gabriela G. Loots 04-ERD-052
}

\begin{abstract}
Progress in deciphering the human genome could be enhanced by improved computational tools and high-throughput experimental strategies. The aim of this program is to develop tools for determining the function of novel human genes found in all sequenced genomes. Because of its importance as a developmental model, the frog genome has been chosen for sequencing by the Joint Genome Institute (JGI). In collaboration with colleagues at UC Berkeley, we propose to develop methods for using frog embryos as a high-throughput system to document functions of conserved human genes with unknown functions. These methods will computationally identify and predict functions for evolutionarily conserved human genes and test those predictions using gene manipulation strategies in frog embryos.
\end{abstract}

Because amphibian and mammalian development is very similar, the main output of this project is a robust set of computational and experimental tools and applications that can be extended to the analysis of complex eukaryotic genomes. In particular, we plan on using these resources to identify and characterize unknown, developmentally expressed human genes that are shared by all vertebrate genomes sequenced to date. These data will be critical to understanding the roles these genes play during evolution, vertebrate development, susceptibility to disease, human health, and interactions with the environment.

\section{Mission Relevance}

Results of this project will have direct applications to bioinformatics, functional genomics, and other biological sciences in support of LLNL's mission in biotechnology for improving human health. In addition, the project fosters strong collaborations with JGI and UC Berkeley in the field of computational biology. 


\title{
FY06 Accomplishments and Results
}

In FY06 we (1) aligned the frog and human genomes and created a complete catalog of conserved elements; (2) created a database that catalogs novel genes and their expression patterns, provides information on gene function, and establishes links to sequence annotation and evolutionary profiles through a browser; (3) determined the expression pattern and function of 50 novel genes; and (4) developed high-throughput automation to scale this process up to whole-genome analysis. This project has created a much-needed infrastructure for the genome-scale, in vivo analysis of a novel vertebrate animal model, the frog Xenopus tropicalis.

\section{Publications}

Loots, G. G., and I. Ovcharenko, in press. "ECRBase." BMC Bioinformatics. UCRL-JRNL-223593.

Loots, G. G., et al., 2006. "Array2Bio: from microarray expression data to functional annotation of co-regulated genes." BMC Bioinformatics. 16, 307. UCRL-JRNL-219041.

\section{Characterizing the Regulatory Genome: Transcription Factor Proteins and Gene Regulation Networks in Living Cells}

Lisa J. Stubbs

04-ERD-084

\begin{abstract}
This research seeks to establish new strategies and technologies for characterizing transcription factor (TF) proteins and the regulatory pathways in which they participate within living cells. Understanding TF proteins and their regulatory "targets" is key to constructing regulatory network models, an important goal in current efforts by both National Institutes of Health and DOE biology programs, including Genomics: GtL. Beginning with a subclass of human TF proteins, we will develop a pipeline for characterizing them and their pathways, with the goal of building robust regulatory network models. All basic methods and expertise will be fully extensible to regulatory network modeling in any species, from microbes to mammals.
\end{abstract}

This project has already succeeded in (1) developing novel methods for identifying TF protein binding sites and target genes in living cells, and for clustering TF proteins and target genes into metabolic, morphological, and developmental pathways; (2) recruiting postdoctoral scientists with state-of-the-art expertise to add depth and breadth to genetics, comparative genomics, biochemical technology, and bioinformatics at LLNL; and (3) developing collaborative ties to university researchers who are leaders in this field. If work in this project's final year is successful, we expect to generate and publish data on key proteins to set the stage for future modeling of regulatory networks involved in reproduction, immune development, and susceptibility to cancer. 


\section{Mission Relevance}

Accurate models of gene regulatory networks are key to understanding biological mechanisms that govern form and function in all types of living cells. By building expertise in regulatory biology, biochemistry, and network modeling, this project supports Laboratory missions in biodefense, environmental management, and bioscience to improve human health.

\section{FY06 Accomplishments and Results}

In FY06, we (1) completed development of robust methods for TF protein target identification; (2) applied our novel methods to identify gene targets for 15 TF proteins, and made significant headway on studies of five additional TF proteins; (3) published two major manuscripts on characterization of this TF protein family; (4) developed a method for in vivo tagging of TF proteins in human cells; and (5) discovered important new links between ZNF proteins and human development, immune system function, angiogenesis pathways, and cancer. These data have provided the first look at the health-related functions of this large human TF family.

\section{Proposed Work for FY07}

In FY07, we will (1) complete analysis of proteins with relevance and potential health applications; (2) confirm identified TF protein targets and pathways; (3) explore TF gene variation to identify heritable factors that may underlie individual differences in development and disease susceptibility; and (4) complete a novel technology for TF protein tagging, and publish the results.

\section{Publications}

Hamilton, A. T., et al., 2006. "Evolutionary expansion and divergence in the ZNF91 subfamily of primate-specific zinc finger genes." Genome Res. 16(584).

UCRL-JRNL-215844.

Huntley, S., et al., 2006. "A comprehensive catalog of human KRAB-associated zinc finger genes: Insights to the evolutionary history of a large family of transcriptional repressors." Genome Res. 16(669). UCRL-JRNL-216004. 


\title{
De Novo Identification of Regulatory Regions in Intergenic Spaces of Prokaryotic Genomes
}

\author{
Patrick S. Chain
}

04-ERD-103

\begin{abstract}
This project will implement, test, and experimentally validate the results of an algorithm for genome-wide identification of candidate transcription-factor binding sites in prokaryotes. Most techniques used to identify regulatory regions rely on conservation between different genomes or have a predetermined sequence motif to perform a genome-wide search. Such techniques cannot be used with new genome sequences. This project will apply a de novo search algorithm to identify candidate binding-site motifs in intergenic regions of prokaryotic organisms, initially testing the available genomes of the Yersinia genus. We will retrofit existing nucleotide pattern-matching algorithms, analyze the candidate sites and their target genes for meaningful patterns, and test these sites experimentally.
\end{abstract}

Using properly annotated prokaryotic genomes, this project will produce a set of software applications capable of identifying candidate intergenic sites important for gene regulation. We will demonstrate this in Yersinia, a model biodefense, Category A group of pathogens, and follow up with experimental evidence that these regions are indeed involved in regulation. The ability to quickly characterize transcription-factor binding sites will lead to increased understanding of how virulence pathways are modulated in biodefense-related organisms, and will help our understanding and exploration of regulons-gene regulatory networks-and novel pathways for metabolic processes in environmental microbes.

\section{Mission Relevance}

The novel search algorithm developed in this project will provide an important tool for genome, transcriptome, and systems biology. It will allow scientists to explore gene regulation to better understand regulatory networks in prokaryotic organisms, and to apply this pathway information to those microbes relevant to the Laboratory's environmental and biodefense missions.

\section{FY06 Accomplishments and Results}

In FY06, we identified a number of putative transcription-factor binding sites by analyzing microarray data and searching the promoters of co-regulated genes for conserved DNA regions. These included rpoN (ntrA), FNR, icIR, and several other binding sites. Multiple alignments of all available genomes for Yersinia confirmed that intergenic regions will be conserved only among members of a species, with divergence seen between orthologous genes in different species of Yersinia. For experimental validation of both the binding sites as well as the regulators, a set of vectors suitable for use in Yersinia were tested and can be used for generating targeted mutations in Yersinia pestis and Yersinia pseudotuberculosis. Future work can build on this LDRD research by disrupting putative binding sites and their transcription factors, and observing their effect on the Yersinia phenotype. 


\title{
Time-of-Flight, Secondary Ion Mass Spectrometry Measurement of Metabolites from Single Cells
}

\author{
Kristen S. Kulp \\ 04-ERD-104
}

\begin{abstract}
Previous studies of microbial response to environmental stress have shown that adaptive response is reflected in changes in the most abundant metabolites. We propose to use time-of-flight, secondary-ion mass spectrometry (ToF-SIMS) to characterize metabolites in individual bacteria to facilitate modeling of metabolite fluxes. ToF-SIMS is a mass spectrometry technique that is used to characterize chemical composition and can produce chemical maps of the distribution of small molecules with the spatial resolution needed to interrogate single cells. Our goal is to enhance bio-analytical instrument capabilities, perfect sample preparation methods, and apply statistical analysis to provide the sensitivity needed to measure bacterial metabolites in single cells.

This research will develop an unexplored area of imaging mass spectrometry: characterization of metabolites from an individual bacterium. This proposal will result in (1) quantitative demonstration of enhanced ToF-SIMS instrument sensitivity, (2) a reproducible sample preparation method that renders bacterial metabolites accessible for analysis, and (3) a useful, multivariate spectral pattern-recognition technique that can compare metabolite production from multiple individual cells. These experiments will be the first-ever analysis of bacterial metabolites in single cells. This work will provide preliminary data to understand pathway fluxes and population interactions of environmentally stressed bacteria.
\end{abstract}

\section{Mission Relevance}

This project will provide the foundation for single-cell metabolomics and biological imaging, in support of LLNL's mission in bioscience to improve human health. This work also is relevant to DOE's Genomics:GtL initiative by developing the capability to measure metabolites in a single bacterial cell, and complements efforts to study spectral signature definition and intracellular compound localization in mammalian cells.

\section{FY06 Accomplishments and Results}

In FY06, we (1) successfully completed and tested the molecular beam vacuum chamber for matrix deposition, (2) determined fragmentation patterns of metabolite standards, (3) implemented the crushing method for exposing metabolites from bacteria, (4) demonstrated superb reproducibility in spectra taken over several months, (5) established a microscope platform that permits microvolume solvent delivery with a micromanipulator that can be used to prepare single cells for planar chromatography to separate small-molecule metabolites from large cell fragments, (6) used statistical analysis techniques to identify key masses in the spectra from different samples, and (7) compared these masses with the metabolite spectral library to identify the origin of important mass fragments. 


\title{
Proposed Work for FY07
}

In FY07, we propose to (1) determine detection limits for standards spiked into bacterial cell homogenates, (2) explore alternative matrices to enhance ToF-SIMS detection sensitivity and detectable mass range, (3) apply a massive cluster-ion source such as buckminsterfullerene as the primary source for ToF-SIMS analysis, (4) utilize substrateenhanced SIMS to improve ion signals from bacteria, (5) further develop our innovative single-cell chromatography using nano-pillar chips, (6) apply supervised chemometric pattern-recognition and classification techniques to extract additional relevant biological information from the data sets, and (7) analyze model cell populations under different growth conditions to understand changes in metabolite production.

\section{Publications}

Berman, E. S. F., et al., 2006. Distinguishing stereo and structural isomers with time-offlight secondary ion mass spectrometry (ToF-SIMS) and principal component analysis. American Society for Mass Spectrometry, Seattle, WA, May 2006. UCRL-POST-221597.

Berman, E. S. F., et al., in press. "Distinguishing monosaccharide stereo and structural isomers with ToF-SIMS and multivariate statistical analysis." Anal. Chem.

UCRL-JRNL-221140.

Kulp, K. S., et al., 2006. Chemical mapping and cell type differentiation of paraffinembedded tissue sections using imaging mass spectrometry. American Association for Cancer Research, Washington, D.C., Apr. 2006. UCRL-ABS-218670.

Kulp, K. S., et al., 2006. "Chemical and biological differentiation of three human breast cancer cell types using time-of-flight secondary ion mass spectrometry (ToF-SIMS)." Anal. Chem. 78, 6351-6358. UCRL-JRNL-218036.

Wu, L., et al., in press. "Imaging and differentiation of mouse embryo tissues by ToF-SIMS." Int. J. Mass Spectrom. UCRL-JRNL-222584.

\section{Calcium Dynamics in Human Bone}

Darren J. Hillegonds 04-ERI-009

\begin{abstract}
Previous work has shown the value of calcium- $41\left({ }^{41} \mathrm{Ca}\right)$ accelerator mass spectrometry in tracking bone health, primarily related to osteoporosis. This proposal broadens the ${ }^{41} \mathrm{Ca}$ assay to include impact on cancer treatment and survival. Our goal is to develop a minimally invasive method to detect and quantify perturbations in the turnover of bone when cancer spreads to the skeleton. Such an early-detection protocol could vastly improve patient quality of life and survival by allowing the use of anticancer therapies prior to significant skeletal damage. We will also develop the ability to closely monitor the progression of skeletal tumor growth, which could provide for significant reductions in the debilitating consequences associated with this disease.
\end{abstract}


Our deliverable will be a minimally invasive method to detect the transition from the primary cancer site to the skeleton, as well as the ability to closely monitor the progression of skeletal tumor growth.

\section{Mission Relevance}

By developing a minimally invasive method to detect the transition from a primary cancer site to the skeleton, this project will further Lawrence Livermore's mission in bioscience and technology to improve human health and will support the National Institutes of Health and National Cancer Institute missions.

\section{FY06 Accomplishments and Results}

In FY06, we completed development of the assay for human subjects and showed that it is more effective than other techniques because there is no apparent diurnal variability in the urinary ${ }^{41} \mathrm{Ca} / \mathrm{Ca}$ signal and this signal varies by less than $4 \%$ over a 48 -hour period. We also demonstrated that the ${ }^{41} \mathrm{Ca}$ assay provides a measure of bone destruction from cancer growth in bone, and that the ${ }^{41} \mathrm{Ca}$ signal is highly correlated with other measurements of both bone turnover and cancer cell proliferation. We met the objective of this LDRD by demonstrating that the ${ }^{41} \mathrm{Ca}$ assay is the most sensitive method available of measuring bone turnover in humans.

\section{Publications}

Denk, E., et al., in press. "Labeling of the human skeleton with ${ }^{41} \mathrm{Ca}$ for the assessment of changes in bone calcium metabolism." Anal. Bioanal. Chem. UCRL-JRNL-217923-DRAFT.

Fitzgerald, R. L., et al., 2005. " ${ }^{41} \mathrm{Ca}$ and accelerator mass spectrometry to monitor calcium metabolism in end stage renal disease patients." Clin. Chem. 51(11), 2095.

UCRL-JRNL-217924.

Yang, M., et al., 2006. "The bisphosphonate olpadronate inhibits skeletal prostate cancer progression in a green fluorescent protein nude mouse model." Clin. Canc. Res. 12(8), 2602. UCRL-JRNL-217925-DRAFT.

\section{Single-Cell-Level Investigation of Cytoskeletal Response to External Stimuli}
Amy L. Hiddessen
04-ERI-015

\section{Abstract}

This research investigates the molecular mechanisms that regulate cellular behavior in response to chemical signals. Studies of cytoskeletal response to external signals currently 
suffer from a lack of physical tools to maximize output from traditional bioassays. This project aims to create registered arrays of numerous single or small clusters of living mammalian cells that can be controllably exposed to signals and simultaneously tracked and time-lapse imaged for analysis. We will develop a new chemical patterning method for arraying cells and demonstrate signal delivery to these cells. When applied to conventional bioassays, these arrays will provide better controlled, higher-throughput capability for systematic analysis of cell signaling in many biosystems, such as pathogen-host cell systems, and generate experimental data that will aid computational models for predicting cellular behavior.

A new experimental capability for systematic analysis of single-cell processes will be established, while experimental data gathered could further the development of computational approaches designed to predict cellular behavior. Applications of the technology developed in this work will elucidate new cell signaling pathways that will contribute to a better understanding of both health and disease. In addition, this technology could aid the development of drugs or other therapies for preventing and treating disease.

\section{Mission Relevance}

By developing a new method for systematically examining the behavior of single cells in response to chemical and pathogenic signals, this work will contribute to Lawrence Livermore's homeland security and public health missions, as well as its mission to pursue breakthrough scientific research. This research also strengthens our collaborative relationship with the University of California.

\section{FY06 Accomplishments and Results}

In FY06, we (1) developed a new chemical method to construct arrays of single or small clusters of mammalian cells; (2) engineered protein/antibody chemistries to array adherent cells, such as epithelial cells, to study pathogen-host cell interactions; (3) used fatty-acid chemistry to array non-adherent adult stem cells for the first time; (4) achieved controlled delivery of signals to, and subsequent time-lapse observation of, live arrayed cells to demonstrate our capability to study cell response to signals; and (5) were first to establish preliminary assays to knockout genes in a specific adult stem-cell type for a collaborative study with the University of California. Overall, we successfully developed a capability for analyzing single-cell behavior in response to external stimuli, and have given several international talks and are currently preparing journal articles reporting our results.

\section{Publications}

Hiddessen, A. L., and J. P. Bearinger, 2006. Chemically patterned, biocompatible substrates for cellular analysis. Biophysical Society Ann. Mtg., Salt Lake City, Utah, Feb. 18-22, 2006. UCRL-ABS-218738. 


\title{
A Single-Molecule Study of the Movement of a DNA Sliding Clamp
}

Daniel Barsky

\author{
04-LW-069
}

\begin{abstract}
We propose a combined experimental and computational project to investigate the interaction between DNA and a sliding-clamp protein. By encircling DNA, slidingclamp proteins have been thought to freely slide along DNA, a movement that defies expectations because the protein's high positive charge should create a strong electrostatic attraction to the DNA. It is also mysterious that the clamp proteins can proceed past sizeable distortions in DNA. By single-molecule fluorescent energy transfer (FRET) experiments, analytical calculations, and molecular dynamics simulations, we are studying the details of the motions of the bacterial sliding clamp along DNA, including identification of key protein residues as well as clamp speed and clamp loading and unloading kinetics.

Using high-end computing, analytical theory, state-of-the-art biochemistry, and singlemolecule fluorescence measurements, this project endeavors to translate the atomic-scale description of biomolecular interactions to nanometer- and micrometer-scale interactions, an important feat in chemistry and material science. Moreover, this project has the potential to answer some fundamental scientific questions, such as how the ubiquitous DNA sliding clamp actually functions in DNA replication and repair. Through this work, many details of the interactions between two dynamically interacting biomolecules will be revealed. The proposed project brings together computations and experiment in a new way that holds great promise in biology, chemistry, and material science.
\end{abstract}

\section{Mission Relevance}

By answering fundamental questions in biology concerning the details of DNA replication and repair, this project supports DOE and LLNL's mission in bioscience to improve human health, and has possible applications relevant to the homeland security mission to prevent and counter bioterrorism.

\section{FY06 Accomplishments and Results}

In FY06, we combined molecular dynamics simulations, bioinformatics, and fluorescence correlation spectroscopy with FRET experiments to measure the translation speed of a DNA sliding clamp on plasmid DNA, and to identify the mechanisms and evolutionary features of these DNA-protein interactions. Our first goal was achieved, and significant attraction between the sliding clamp and another key protein was also discovered. The simulations and bioinformatics identified a handful of key protein residues that interact with DNA-two of these residues were recently observed experimentally by x-ray crystallography. We have presented this work at conferences, and will soon publish our results in high-visibility, peer-reviewed journals. 


\title{
Publications
}

Barsky, D., et al., 2006. Single molecule fret and simulations of a DNA sliding clamp moving along DNA. 50th Biophysical Society Mtg., Salt Lake City, UT, Feb. 18-22, 2006. UCRL-ABS-216000.

Laurence, T. A., et al., 2006. Single molecule FRET and simulations of a DNA sliding clamp moving along DNA. 232nd American Chemical Society's National Mtg. and Exposition: Frontiers in Single-Molecule Biophysical Chemistry, San Francisco, CA, Sept. 10-14, 2006. UCRL-PRES-224365.

\section{Emerging Contaminants: Application of Microarray Technology to the Detection of Mixtures of Endocrine-Active Agents}

\author{
Linda C. Hall 05-ERD-008
}

\begin{abstract}
The health effects of previously unidentified, unrecognized, or novel environmental contaminants are a prominent concern of regulatory agencies and the general public. Of particular interest are endocrine-disrupting chemicals (EDCs), including hormone agonists and antagonists, which pose a potential risk to surface water and groundwater due to wastewater treatment facility and septic system discharges. The number and structural diversity of EDCs makes it impractical to develop detection methods on a chemical-bychemical basis. This project proposes to use DNA microarray technology and synchrotron radiation-based Fourier transform infrared (IR) spectromicroscopy to develop a biological sensing system to detect unknown EDCs present individually or as mixtures in water.

Principal results of this research will be a validated sensing system (bioassay) capable of detecting both known and novel EDCs that are present in ambient water individually or as mixtures. We will (1) characterize a human cell line and demonstrate the responsiveness of that cell line to the major categories of EDCs, (2) acquire novel and fundamental knowledge of the timing of gene alteration in response to EDCs, (3) characterize genetic response profiles (individual genes and gene pathways) for EDCs using microarray-based analyses of exposed cells, (4) define the sensitivity and dose-response of microarray techniques, (5) and apply pattern-recognition algorithms to gene expression profiles to identify chemical- and mixture-specific expression patterns.
\end{abstract}

\section{Mission Relevance}

The proposed research combines state-of-the-art technologies of gene expression microarrays and IR spectromicroscopy as the basis of a biological assay to detect mixtures of unknown EDCs in ambient water. This measurement tool is based on novel and fundamental scientific data that addresses a water contamination problem of both statewide and national interest, and supports LLNL's missions in environmental protection and biotechnology to improve human health. 


\title{
FY06 Accomplishments and Results
}

We (1) completed the bioinformatics analysis of cells exposed to two EDC agonists and began work on the third and last agonist; (2) submitted a manuscript based on project results to date; (3) completed range-finding experiments and exposures of cells to three antagonist EDCs, as well as for agonist-antagonist mixtures; and (4) identified expression profiles and gene pathways affected by the chemicals studied to date. However, the IR analysis was set aside due to unanticipated technical problems.

\section{Proposed Work for FY07}

In FY07 we will (1) conduct microarray analyses of RNA from cells exposed in FY06 to single EDCs and EDC mixtures; (2) complete bioinformatics analyses of these microarray results, with an emphasis on identification of compound-specific patterns of gene expression; (3) complete expression profiles and gene pathways affected by the remaining chemicals; (4) finish verification, using quantitative polymerase chain reaction, of expression levels of select marker genes from each microarray experiment; (5) conduct field tests that will complement remaining experimental work to yield a validated system capable of detecting EDCs in ambient water; and (6) publish additional manuscripts.

\section{Publications}

Hall, L., et al., in press. "Effects of thyroid hormones on breast cancer cell proliferation." Endocrinology. UCRL-JRNL-218037.

\section{An Innovative Copolymer Complex to Inhibit Transport of Biological Aerosols}

Paula W. Krauter 05-ERD-027

\begin{abstract}
We are formulating and optimizing polymer solutions for immobilizing and suppressing hazardous biological particle resuspension and migration. Building on our development of a particle-binding solution specific to aerosolized spores, we intend to evaluate filmforming copolymers that have multiple functional groups capable of attracting and binding particles. These solutions will be evaluated for their adhesion to biothreat agents in a series of aerosol experiments, and our research will focus on spore deposition, resuspension under turbulent airflow, and spore attraction and encapsulation.
\end{abstract}

This project will enable a new decontamination tool for emergency responders exposed to biothreat agents. We expect to develop (1) a copolymer-based solution formulated to be anionic to provide coulombic attraction to cationic spores; (2) publications in peer-review journals regarding development, characterization, and evaluations of the formulations; and (3) a deeper understanding of how to limit resuspension of contaminant particles from copolymer application. We also will advance the basic understanding of how particle shear 
stress and particle-copolymer adhesion strength under a variety of flow conditions impact transport of bioaerosols.

\section{Mission Relevance}

The infectious properties of biothreat agents require development of new and effective methods to rapidly immobilize particulates. This project addresses the national security mission to counter proliferation and the use of weapons of mass destruction by developing a new technology to inhibit effective dispersal and migration of biological warfare agents. This will increase the safety of emergency response personnel by abating breathing zone concentrations, as well as limit the potential for spore resuspension and migration.

\section{FY06 Accomplishments and Results}

In FY06, we (1) selected the best copolymer formulation from a field of 11 potential copolymers based on chemical characterization, and measured particle-copolymer solution-binding characteristics in wind-tunnel tests using weaponized Bacillus anthracis surrogates; (2) designed and built a room-size antistatic aerosol chamber on a military base to evaluate particle-copolymer adhesion strength; (3) successfully measured the change in airborne endospore concentration with optical and microbiological instruments; and (4) tested the copolymer-based spray in several environmental conditions to evaluate aerosol knock-down and spore resuspension from surfaces. Overall, this research project provided input for a finite aerosol-reaerosolization model used in evaluating copolymerbased sprays, and resulted in a patent for a hazardous particle binder, coagulant, and reaerosolization inhibitor.

\section{Publications}

Krauter, P. A., 2006. The problem with decontamination agent application: bioparticulate target-hit or miss? Denver Decon Science and Technology Conf., Westminster, CO, Oct. 31-Nov. 7, 2006. UCRL-ABS-223980.

Krauter, P. A., 2006. Spore contamination-what concentration deposits, what resuspends, and can we inhibit its transport? U.S. Environmental Protection Agency Decontamination Workshop at National Homeland Security Research Center, Washington, D.C., Feb. 15-18, 2006. UCRL-PRES-221011.

Krauter, P. A., A. H. Biermann, and L. D. Larsen, 2006. Spore reaerosolization in ventilation ducts. American Society for Microbiology General Mtg., Orlando, FL, May 21-25, 2006. UCRL-ABS-218098.

Krauter, P. A., A. H. Biermann, and L. D. Larsen, 2006. "Spore transport and deposition in ventilation ducts." Proc. American Society for Microbiology Biodefense Research Mtg. UCRL-POST-210650.

Krauter, P. A., A. H. Biermann, and L. D. Larsen. 2006. "Transport efficiency and deposition velocity of fluidized spores in ventilation ducts." Aerobiologia 21, 155. UCRL-JRNL-203868. 
Zalk, D. M., and P. A. Krauter, 2005. Inhibiting the transport of terrorism-related hazardous aerosols to aid decontamination efforts and prevent reaerosolization. 6th Intl. Scientific Conf. Intl. Occupational Hygiene Association, Pilanesberg National Park, North West Province, South Africa, Sept. 19-23, 2005. UCRL-PRES-215323.

\title{
Characterizing Hypothetical Proteins
}

\author{
Michael P. Thelen 05-ERD-064
}

\begin{abstract}
Nearly half the proteins inferred from microbial genome sequences are unconfirmed and bear little resemblance to known proteins. Yet many of these "hypothetical proteins" are important in nature. We propose to establish functions for a significant fraction of hypothetical proteins from an uncultivated microbial community by analyzing protein complexes and the sequences that encode them. We will determine protein distributions in a biofilm community by fractionation and proteome analysis, identify source organisms and genome context, and measure protein abundance. To characterize unknown proteins, we will analyze protein sequences to predict functions, isolate multiprotein complexes, and test predicted functions using parallel biochemical assays.

This research will advance our understanding of how microbes act in their natural environment, particularly the molecular mechanisms of iron oxidation, biofilm formation, and evolution of adaptive mechanisms such as acid tolerance. We will develop new analytical methods, find novel genes, and determine the function of previously unknown proteins, all of which will serve to accelerate environmental research and enhance the scientific basis for LLNL's current focus on a variety of microbes. Important insights into protein function will result from computational modeling. Furthermore, this project will establish unique expertise in the emerging area of proteogenomic analysis, and continue to build collaborations with researchers at UC Berkeley for expanded research in support of the DOE Genomics: GtL program initiative.
\end{abstract}

\section{Mission Relevance}

This project will support LLNL's environmental management mission by developing new approaches to understanding natural microbial systems that influence metal-contaminated environments. It also supports the energy security mission because these microbial systems exacerbate environmental problems caused by mining principle energy sources (e.g., coal and uranium). This will provide an excellent starting point for future work in biosciences and environmental science.

\section{FY06 Accomplishments and Results}

In FY06 we (1) produced new immunoreagents for novel cytochrome proteins of a bacterial member of our model microbial community; (2) used confocal microscopy 
to examine these antibodies and species-specific DNA probes, determining the threedimensional architecture of a biofilm and examining biochemical functions in some of the novel proteins purified from extracellular and membrane fractions; (3) examined the membrane cytochrome under different $\mathrm{pH}$ and oxidation conditions using visible-spectra, circular dichroism and iron redox reactivity; and (4) studied genetic recombination between two strains of a bacterial member of the biofilm by analyzing differences in protein sequences between the genome sequences of the recombinant and the two strains, providing the first comprehensive analyses of a complex, natural community.

\section{Proposed Work for FY07}

In FY07 we will (1) focus on a subset of extracellular proteins in several different biofilm communities to identify variants of these proteins and to determine how they are affected by growth stage, geochemical conditions, and seasonal variations; (2) develop assays to characterize the activities of several proteins, using predictions from structural modeling to guide the choice of substrates; (3) use LLNL's newly established lipid nano-disk technology in a novel test of the stability of the membrane cytochrome and its reactivity with iron; and (4) continue the ongoing, large-scale examination of protein sequences, comparing similar sequences from several acidophilic and neutrophilic organisms for specific structural clues to mechanisms of acid tolerance.

\section{Publications}

Banfield, J. F., et al., 2005. "Proteogenomic approaches for the molecular characterization of natural microbial communities." OMICS: J. Integr. Biol. 9, 301. UCRL-JRNL-218040.

Beernink, P. T., et al., 2006. "Specificity of protein interactions mediated by brct domains." J. Biol. Chem. 280, 30206. UCRL-JRNL-218035.

Jeans, C., et al., 2006. Investigating novel proteins in acidophilic biofilm communities. Genomics: GTL Contractor-Grantee Workshop IV, North Bethesda MD, Feb. 12-15, 2006. UCRL-POST-218849.

Jeans, C., et al., in press. "Purification and characterization of a novel outer membrane cytochrome, the iron oxidase of leptospirillum group II." Appl. Environ. Microbiol.

UCRL-JRNL-225478.

Lo, I., et al., in press. "Strain-resolved community proteomics reveals that recombination shapes the genomes of acidophilic bacteria." Nature. UCRL-JRNL-225470.

Ram, R. J., et al., 2006. "Community proteomics of a natural microbial biofilm." Science 308, 1915. UCRL-JRNL-218041.

Siebers, A. K., S. W. Singer, and M. P. Thelen, 2006. Analyzing the structure and function of novel cytochromes from a natural microbial community. UCRL-TR-224023.

Singer, S. W., et al., 2006. Unique Fe(II)-oxidizing cytochromes isolated from acidophilic biofilms. Gordon Research Conf. Environmental Bioinorganic Chemistry, Andover, NH, June 18-23, 2006. UCRL-POST-222105. 
Thelen, M. P., 2006. Biofilm proteins associated with acid mine drainage. American Society for Microbiology Conf., Orlando, FL, May 19-21, 2006. UCRL-PRES-225449.

Wong, S., C. Jeans, and M. P. Thelen, 2006. A study of the structure and metabolic processes of a novel membrane cytochrome in an extreme microbial community. UCRL-TR-224396.

\title{
Comparative Analysis of Genome Composition with Respect to Metabolic Capabilities and Regulatory Mechanisms
}

Patrik M. D'Haeseleer 05-ERD-065

\begin{abstract}
Given the glut of sequence data, comparative genomics is essential for leveraging existing knowledge. However, most approaches are limited to closely related species. We intend to link genome content to phenotype across hundreds of fully sequenced organisms. By studying a large collection of bacterial genomes at the level of genome content rather than precise sequence similarity, and integrating data on gene function and species phenotype, we intend to elucidate genotype-to-phenotype mapping, with particular emphasis on metabolic processes and regulatory mechanisms. Our modeling tools to decompose the genome composition will include non-negative matrix factorization, spectral methods, classification and regression models, and other machine learning techniques.
\end{abstract}

The patterns we discover in gene composition across the spectrum of bacterial genomes will increase understanding of which genes, gene classes, pathways, etc. are associated with or required for specific bacterial phenotypes, as well as yielding computational predictions of function for many unknown genes. Based on a list of genes in a newly sequenced genome (or even an unassembled environmental "shotgun" sequence), we expect to predict the metabolic processes and regulatory mechanisms and how these regulatory mechanisms respond to perturbations, which will give us insight on modifying or exploiting the organism(s) in question. Such a predictive capability for genotype-tophenotype mapping is crucial for analyzing the flood of new sequence data.

\section{Mission Relevance}

The predictive capability for genotype-to-phenotype mapping created in this project will address goals of DOE's programs in biological and environmental science to gain predictive mastery of the microbial world. Future applications of the capability will support LLNL's missions in homeland security, environmental assessment and management, and biosciences to improve human health.

\section{FY06 Accomplishments and Results}

In FY06, we (1) assembled a BioWarehouse database that encompasses genomes, taxonomy, bacterial genes and proteins, metabolic pathways, and phenotypes; (2) created 
a statistical phenotype model that uses genomic data to predict whether a genome represents a human and/or animal pathogen, and its optimal growth temperature, outer membrane type, type III secretion, and type of flagella; (3) examined sets of genes linked to specific phenotypes, as well as a gene set involved in determining both optimal growth temperature and pathogenicity of a bacterial pathogen; and (4) began gathering transcription factor data from Genbank and other databases. Mapping of transcriptionfactor binding sites in Escherichia coli have been delayed because of the departure of a collaborator.

\title{
Proposed Work for FY07
}

In FY07, we will (1) continue to pursue a possible collaboration with Biolog for use of their microbial identification database, a uniform set of 95 phenotypic descriptors across more than 1900 species; (2) explore machine learning techniques for genome decomposition for prediction of phenotypes; (3) analyze a number of gene sets correlated with metabolic phenotypes of interest; (4) apply phenotype prediction to metagenomic samples; (5) prepare a paper on genome decomposition; (6) complete the transcription factor and transcription-factor binding site compendium; (7) evaluate transcription-factor binding site prediction algorithms and design a novel algorithm, if necessary; and (8) explore the feasibility of applying the motif-expression decomposition method to a number of model organisms and extrapolating it to non-model organisms.

\section{Publications}

D'Haeseleer, P., 2006. "What are DNA sequence motifs?" Nat. Biotechnol. 24, 423. UCRL-JRNL-218978.

\section{Development of a Chemoenzymatic-Like and Photoswitchable Method for the Ordered Attachment of Proteins to Surfaces}

\author{
Julio A. Camarero 05-LW-018
}

\begin{abstract}
Protein arrays are ideal tools for rapidly analyzing whole proteomes as well as for developing reliable and cheap biosensors. The objective of this proposal is to develop a new entropically activated ligation method based on the protein trans-splicing process. This method will be used to generate spatially addressable arrays of multiple protein components by standard photolithographic techniques. The key to our approach is the use of protein trans-splicing, a naturally occurring process that will allow us to create a truly generic and highly efficient method for the covalent attachment of proteins through its C-terminus to any solid support.
\end{abstract}

We expect to achieve selective and efficient attachment of any protein to solid supports through its C-terminus. Because this process is highly selective, protein immobilization will not require the prior purification of the proteins. Chemical modification of the C-intein 
polypeptide with photocleavable protecting will allow us to use standard photolithographic techniques to create protein chips with thousands of different protein combinations. These chips could be used for proteomic analysis, drug discovery, and biosensing.

\section{Mission Relevance}

Because protein arrays are very efficient reagents for the parallel analysis of whole proteomes, the technology developed in the project will support LLNL missions in national security, homeland security, and bioscience and technology to improve human health. In addition, developing new methods for the rapid and efficient creation of protein chips is one of the top priorities of DOE's Genomics: GtL initiative.

\section{FY06 Accomplishments and Results}

In FY06, we (1) cloned, expressed, and purified several $\mathrm{N}$-intein fusion proteins, including discosoma red (DsRed) and enhanced green fluorescent protein (EGFP); (2) immobilized DsRed and EGFP onto C-intein-coated glass slides through protein trans-splicing; and (3) produced several photocaged versions of the C-intein polypeptide of the gene DnaE that is able to photomodulate protein attachment through photoactivated protein trans-splicing.

\section{Publications}

Camarero, J. A., and Y. Woo, 2006. "Interfacing soft and hard matter with exquisite chemical control." Curr. Nanoscience 2, 93. UCRL-JRNL-218508.

Camarero, J. A., 2006. "Introduction to polypeptide chemical ligation tools in protein engineering." Protein Pept. Lett. 12, 721. UCRL-JRNL-217815.

Camarero, J. A., 2006. "New developments for the site-specific attachment of proteins to surfaces." Biophys. Rev. Lett. 1, 1. UCRL-JRNL-212352.

Camarero, J. A., Y. Kwon, and M. A. Coleman, 2006. "Selective and traceless immobilization of proteins onto solid supports through protein trans-splicing-application to the rapid production of protein chips." Protein Sci. 15, 235. UCRL-ABS-222781.

Camarero, J. A., Y. Kwon, and M. A. Coleman, 2006. "Selective immobilization of proteins onto solid supports through split-intein mediated protein trans-splicing." Angew. Chem. Int. Ed. 45, 1726. UCRL-JRNL-214820.

Camarero, J. A., Y. Kwon, and M. A. Coleman, 2006. "New tools for the site-specific attachment of proteins to surface." Biopolymers 80, 499. UCRL-CONF-222518. 


\title{
Developing and Integrating Novel Technologies for the Production and Characterization of Membrane Proteins
}

Paul D. Hoeprich

06-SI-003

\begin{abstract}
Membrane-associated proteins have received limited study because they are not amenable to conventional techniques for isolating and characterizing soluble proteins. This project will develop robust, state-of-the-art methods to produce and characterize membrane proteins and to synthesize unique molecular-affinity tags for them. Specifically, we will (1) produce membrane proteins by cell-free methods, (2) capture these proteins in nanosized (10- to 30-nm) cell membrane mimetic structures we have termed nanolipoprotein particles (NLPs), (3) optimize NLP production by creating semi-synthetic lipoprotein molecules, (4) characterize NLP constructs, and (5) synthesize molecular-affinity tags to identify membrane proteins contained in NLPs.

This project will result in generally applicable methods for the production, isolation, characterization, and functional reconstitution of membrane-bound proteins and protein complexes in NLPs. Success will significantly benefit life sciences in general and advance biological science at LLNL, while providing a better understanding of cell membraneassociated proteins. Following proof-of-principle studies, we will produce and capture membrane proteins relevant to biodefense, such as hemagglutinin $\mathrm{H} 5$. Subsequent work will focus on proteins that play an important role in DOE's strategies for environmental management and energy production. The capabilities we develop for membrane proteins will be adaptable to biodefense, bioenergy, environmental biology, and human health research.
\end{abstract}

\section{Mission Relevance}

This work supports LLNL missions in biodefense, energy security, and environmental management. NLPs can serve as carriers of non-infective immunogenic proteins like hemaggultinin $\mathrm{H} 5$ protein from avain influenza-such a construct could result in vaccinebased countermeasures. Membrane-associated cellulase complexes and hydrogenase enzymes in NLPs could be used to explore and understand conversion of cellulosic biomass to transportation fuel and produce hydrogen, respectively. Understanding molecular aspects of uraninite oxidation by Thiobacillus denitrificans will contribute to better bioremediation processes for uranium. Overall, this work will support LLNL efforts in fundamental life sciences and biotechnology.

\section{FY06 Accomplishments and Results}

In FY06, we (1) used cell-free protein synthesis to express 3 different lipoproteins (scaffold proteins) and 11 membrane proteins, each targeted for reconstitution in NLPs;

(2) commenced lipoprotein (scaffold protein) scale-up synthesis in cell-based systems; 
(3) created NLPs with three different lipoproteins and two types of phospholipids;

(4) characterized NLP constructs by size-exclusion chromatography, gel electrophoresis, small-angle $x$-ray scattering, transmission electron microscopy, ion mobility spectrometry, and atomic force microscopy; (5) obtained preliminary results suggesting strongly that we have made NLPs containing the functional membrane protein bacteriorhodopsin (bR); and (6) produced affinity tags (DNA aptamers) against a model target protein.

\title{
Proposed Work for FY07
}

In FY07, we will (1) initiate biochemical characterization of membrane proteins and affinity tags of interest to biodefense; (2) continue scaffold protein production, including insect lipophorins; (3) commence protein engineering using chemical synthesis of helical peptide segments ligated to protein segments produced biochemically to create novel scaffold proteins; (4) continue NLP production, using more diverse approaches with assorted phospholipids; (5) develop optimal methods for membrane protein incorporation into NLPs, including semi-automated approaches; and (6) continue cell-free synthesis of membrane proteins such as influenza hemagglutins for inclusion into NLPs. These activities will lead to state-of-the-art methods to produce and characterize membrane proteins.

\section{Development of Single-Cell Raman Spectroscopy for Cancer Screening and Therapy Monitoring}

James W. Chan

$$
\text { 06-ERD-051 }
$$

\begin{abstract}
Current methods for determining cancer prognosis are nonspecific and invasive, making the development of better methods for early detection of cancer and its recurrence vital for improved survival rates. Our objective is to develop new techniques for detecting early cancer using single-cell Raman spectroscopy. By making significant advances in the sensitivity and efficiency of Raman technology, and by identifying new Raman markers of cancer, we hope to achieve a high-throughput, single-cell analytical tool. To understand the biology, we will acquire data on different leukemia cell types from clinical and commercial samples, apply algorithms for analysis, and correlate results to clinical parameters.
\end{abstract}

We expect to (1) develop laser-tweezers Raman spectroscopy to characterize unique spectral markers of single, living cancer cells; (2) determine this technique's accuracy and sensitivity; (3) achieve a fundamental understanding of the biological relevance of these spectral markers; and (4) develop an optical trapping, nonlinear Raman technique that is based on coherent anti-Stokes Raman spectroscopy, which should significantly speed up single-cell analysis. The cancer signatures, data algorithms, and Raman technology developed in this project will lead to the development of a novel clinical tool, such as a Raman flow cytometer, that will replace or complement existing technology to improve early detection and diagnosis. 


\section{Mission Relevance}

This project supports the Laboratory's mission in bioscience to improve human health. By developing Raman-based optical techniques for cancer diagnostic and therapy applications, this project will enable more cost-effective, highly specific, early-disease-detection and treatment technology and biomedical instrumentation. In addition, success in this work can impact homeland and national security by defining an approach to analyzing cell biochemistry that could result in high-throughput screening methods for identification of an exposed population following a biological event.

\section{FY06 Accomplishments and Results}

In FY06, we (1) developed, in collaboration with the National Science Foundation's Center for Biophotonics Science and Technology at the University of California at Davis, two laser-trap Raman systems that will enable us to compare the reproducibility of spectral data on multiple systems; (2) developed and implemented a canonical variate analysis (CVA) technique, which showed improved data classification; (3) applied our technique to the analysis of clinical leukemia samples, achieving initial results that showed the ability to differentiate leukemia cells from normal cells; (4) analyzed normal cells in different cell-cycle stages to determine signal heterogeneity and its effect on accurate cancer cell detection; and (5) published our initial work on cultured cells in a peer-reviewed journal.

\section{Proposed Work for FY07}

In FY07, we plan to (1) expand the analysis to a larger patient population with other leukemias to determine the sensitivity limits for single-cancer-cell detection; (2) continue using statistical CVA to analyze and classify the data; (3) perform quantitative analysis of the spectral data by fitting the data to a basis set of pure component (DNA and protein) spectra and extracting information on cellular composition; (4) validate our data by comparing the results of quantitative analysis to clinical analyses (flow cytometry, staining, and blotting) of the samples; and (5) perform Raman spectroscopy on cells in activation, proliferation, and apoptosis to determine biological relevance of the markers and the effect of biological variation on accurate detection of cancer cells.

\section{Publications}

Chan, J. W., et al., 2006. "Micro-Raman spectroscopy detects individual neoplastic and normal hematopoietic cells." Biophys. J. 90, 648. UCRL-JRNL-209131. 


\title{
Francisella Tularensis: Understanding the Host-Pathogen Interaction
}

Amy Rasley

06-ERD-057

\begin{abstract}
The highly infectious nature of Francisella tularensis-the agent that causes tularemia (also known as rabbit fever) in humans-highlights a need for continued research efforts to understand the interactions of this organism with host (human) immune defenses. Very little is known about how this pathogen defeats host immune responses to cause disease, and no licensed vaccine is currently available. This project will investigate factors involved in the early immune response to $F$. tularensis infection. Our goals are to uncover global host-response patterns that may be used for early detection of exposure to this potential biowarfare pathogen, and to understand how $F$. tularensis evades innate immune defenses to cause disease. Proposed work will build on extensive genomic and virulence expertise at the Laboratory.
\end{abstract}

If successful, this project will identify the host genes that are important during F. tularensis infection, and define the post-infection role that host pattern-recognition receptors play in detecting $F$. tularensis and initiating innate immune responses. The proposed research could ultimately lead to the development of efficacious therapies to combat tularemia and may help us identify potential early warning markers for biowarfare agent exposure.

\section{Mission Relevance}

Developing the ability to effectively detect and treat exposure to the highly infectious potential biowarfare agent $F$. tularensis first requires gaining an understanding of how this pathogen interacts with host cells to cause disease. Moreover, by defining this previously unknown pathway of pathogen infection and linking it to the human genomic sequence, we will be advancing the detection of emerging and engineered threats. Consequently, this research directly supports LLNL's national security mission, particularly in the areas of homeland security and biodefense.

\section{FY06 Accomplishments and Results}

In FY06, we (1) optimized culture conditions for mammalian cells, determined optimal multiplicities of infection for $F$. tularensis for our infection studies, and obtained bacterial growth curves for 26 and $37^{\circ} \mathrm{C}$; (2) performed infection studies using mouse macrophages and two strains of $F$. tularensis for RNA isolation and subsequent microarray analyses; (3) began optimizing our microarray labeling and hybridization conditions; (4) obtained preliminary microarray data from in vitro infection experiments of macrophages exposed to the two strains of F. tularensis; and (5) expanded our scope to include gene expression 
analyses of $F$. tularensis during temperature shifts using custom microarrays developed in collaboration with Stanford University researchers.

\title{
Proposed Work for FY07
}

In FY07, we will (1) finalize microarray analyses begun in FY06; (2) compare results obtained from mouse macrophages infected with $F$. tularensis with those obtained from infected human macrophages to assess commonalities in the response to infection, as well as "unique responses" likely to arise from genetic variability within the human population; and (3) investigate levels of expression of messenger RNA (mRNA) and toll-like receptors in human and mouse macrophages infected with $F$. tularensis, then correlate mRNA expression with protein levels via protein immunoblot and fluorescence-activated, cellsorting analyses.

\section{Characterization and Quantification of Dynamic Robustness in Biological Systems}

\author{
Eivind Almaas $\quad$ 06-ERD-061
}

\begin{abstract}
Organisms, even single-celled ones, are extremely complex dynamical systems that must maintain functional stability despite being constantly impacted by destabilizing forces. Recent discoveries demonstrate that a major destabilizing force lies in the stochastic nature of the cellular machinery itself. This proposal aims to (1) develop analytical and computational methods to identify elements in whole-cell network architectures that increase the robustness of that cellular machinery, (2) analyze the weaknesses and failure modes of these robustness-conferring elements, (3) develop new constraint-based approaches to studying cellular networks, and (4) develop and test a new metabolic network model of Yersinia pestis, the organism that causes bubonic plague.

By coupling experimental information on whole-cell organization with analytical and computational predictions and models, we expect to develop a comprehensive understanding of the levels of environmental and inherent internal variation that microbes can tolerate. We hope to infer general principles of how individual microorganisms and communities of microorganisms manage both internal and external stochastic variations to avoid the multitude of potential failure modes. We aim to understand and predict the mechanisms that determine observed failure rates and gain a similar understanding of the dominant failure modalities. For $Y$. pestis, we expect to develop a basic understanding of its metabolic function, as well as identifying genes that could provide targets for new antibiotics.
\end{abstract}




\title{
Mission Relevance
}

By identifying possible genetic targets for antibiotics to use against $Y$. pestis, this project supports Lawrence Livermore's national security mission. More broadly, this project supports the Laboratory's mission in breakthrough science by developing expertise and new methods for the study of stochastic noise propagation in biological networks and the robustness of cellular organisms.

\section{FY06 Accomplishments and Results}

After a midyear FY06 start, we (1) successfully assembled our project team and hired two postdoctoral researchers; (2) began developing a whole-cell metabolic model for $Y$. pestis and $Y$. pseudotuberculosis, and have started the verification process of this first model; (3) studied noise propagation in small gene circuits, analyzing the robustness properties of our theoretical-computational model for a genetic sequential feed-forward loop; and (4) completed computational predictions for epistatic recovery for experiments being performed at Harvard Medical School.

\section{Proposed Work for FY07}

In FY07, we will (1) obtain results from the initial analysis of noise propagation in small gene circuits (such as the feed-forward loop) and develop similar tools applicable to small metabolic modules; (2) verify and expand the whole-cell metabolic model of $Y$. pestis and $Y$. pseudotuberculosis and include simple gene-regulatory modules; (3) submit for publication our predictions on epistasis in both bacteria and eukaryotic metabolic networks; and (4) continue our multidisciplinary exploration of complex cell network methods.

\section{Redox Proteins in Environmentally Relevant Bacteria}

Harry R. Beller

06-ERD-063

\begin{abstract}
This project adopts a systems biology approach that combines genomics, transcriptomics, and proteomics to explore novel, metal-oxidizing reduction-oxidation (redox) reactions that can mediate the behavior of uranium in the subsurface. Our focus is on redox-active proteins that can extract electrons from minerals such as uraninite. This is particularly pertinent to a remediation process of great interest to DOE: the in situ, reductive immobilization of metals, including radionuclides. This project will utilize collaborations with the DOE Joint Genome Institute and leverage LLNL's existing capabilities in genomics, microbiology, protein biochemistry, molecular biology, and geochemistry in a significantly new research direction for bioscience.
\end{abstract}


Expected results include (1) new knowledge about redox-active, outer-membrane proteins in the bacterium Thiobacillus denitrificans, which carries out the anaerobic, nitratedependent oxidation of metals; (2) a complementation system in $T$. denitrificans that can be used to confirm the results of targeted gene mutation (knockout) studies; and (3) a better understanding of the genetic and biochemical basis of anaerobic metal oxidation. These results will be documented through publications in high-visibility journals.

\section{Mission Relevance}

This project furthers Lawrence Livermore's mission in environmental management, particularly the enduring national need for remediation of uranium contamination. The subject of this project-microbial processes active in the near-surface environment-is also relevant to an important national security issue of radionuclide contamination of groundwater.

\section{FY06 Accomplishments and Results}

Major accomplishments in FY06 include (1) achieving the first-ever fractionation of the outer-membrane proteins of $T$. denitrificans using sucrose density-gradient ultracentrifugation; (2) separation of outer-membrane proteins by gel electrophoresis, (3) successfully developing a method to visualize hemoproteins (cytochromes) in electrophoresis gels using enhanced chemiluminescence; (4) generation of knockout mutants of two genes, including a c-cytochrome, potentially involved in anaerobic metal oxidation; and (5) developing a genetic complementation system in $T$. denitrificans to confirm the results of gene knockout experiments. In addition, we prepared a manuscript for a peer-reviewed journal on our whole-genome transcriptional studies of T. denitrificans.

\section{Proposed Work for FY07}

In FY07, we will (1) isolate the outer-membrane proteins expressed by $T$. denitrificans when it carries out anaerobic iron(II) oxidation, (2) visualize and compare outermembrane hemoproteins expressed under denitrifying conditions when $T$. denitrificans is oxidizing thiosulfate versus iron(II), (3) generate further knockout mutants of genes putatively involved in anaerobic metal oxidation and assay the mutants for metal-oxidizing activity, and (4) prepare a manuscript describing the novel genetic system developed for T. denitrificans.

\section{Publications}

Beller, H. R., et al., 2006. "Whole-genome transcriptional analysis of chemolithoautotrophic thiosulfate oxidation by Thiobacillus denitrificans under aerobic vs. denitrifying conditions." J. Bacteriol. 188, 7005. UCRL-JRNL-220798. 


\title{
Modeling the Mechano-Chemistry of NTPases
}

\author{
Jianhua Xing \\ 06-ERI-004
}

\begin{abstract}
Nucleoside $5^{\prime}$-triphosphate-driven proteins (NTPases) transform chemical energy into mechanical motion, and thus are essential for almost every biological process. We propose to develop an appropriate coarse-grained theoretical framework that can describe the mechano-chemical properties of NTPases. In collaboration with researchers at UC Berkeley, we will apply the theoretical framework to the energy-transduction mechanism of the f29 DNA packing motor, DNA clamps, and the assembly dynamics of microtubules. The theoretical methods will provide tools for experimentalists to analyze data and will be a step toward integrating Laboratory-wide efforts to study the dynamics of complex systems with applications of national significance, such as pathogen invasion.

We will provide a general theoretical framework and a computer program for modeling NTPases. The framework will be capable of integrating various experimental results into quantitative and predictive physical models and of providing guidance for further experimental studies. Using the framework, we will construct a mechano-chemical model of the virus-packaging motor based on available single-molecule force measurements. A model for mechano-transduction of the clamp-clamp loader system will be constructed by integrating the coarse-grained modeling methodology with atomistic simulations. We will also propose a coarse-grained model to explain the flat sheet-microtubule transformation.
\end{abstract}

\section{Mission Relevance}

This project supports the Lab's national security mission by developing tools for studying complex systems such as pathogen invasion. More broadly, this project supports LLNL's mission in bioscience and technology to improve human health by furthering our understanding of compounds (NTPases) that are essential for almost every biological process.

\section{FY06 Accomplishments and Results}

After a midyear start, we (1) developed a general computer code and model for the DNA packaging motor, (2) developed theoretical expressions to describe mechanical stress propagation upon substrate binding on protein motors, (3) worked on tensor visualization and application to the DNA clamp-clamp loader system, (4) performed theoretical analysis on a single-molecule protein fluctuation experiment with collaborators at Harvard University, (5) performed studies on the bacterial flagellar motor; and (6) published a manuscript in Physical Review E.

\section{Publications}

Xing, J., and K. S. Kim, 2006. "Protein fluctuations and breakdown of time-scale separation in rate theories." Phys. Rev. E. 74, 061911. UCRL-JRNL-222129. 


\title{
Quantification of DNA Repair Using Accelerator Mass Spectrometry
}

John M. Hinz

06-LW-019

\begin{abstract}
DNA damage, which occurs both spontaneously and from exposure to exogenous agents, is a primary factor in cancer and aging in humans. Currently, we cannot detect the DNA repair associated with low levels of damage. The objective of this study is to use accelerator mass spectrometry to quantify the biological process of DNA repair associated with spontaneous and low-dose-radiation-induced DNA damage. We will quantify the incorporation of carbon-14-labeled thymidine from cellular DNA repair events in cultured human cells. Such measurements will allow, for the first time, determination of the amount of DNA repair resulting from spontaneous damage associated with cell metabolism, as well as from exposure of the cells to low levels of ionizing radiation and ultraviolet light.
\end{abstract}

The success of this study will provide us unprecedented quantitative results of the amount of DNA repair associated with spontaneous metabolic DNA damage that is performed by human cells. In addition, we will quantify the previously undetectable DNA repair associated with low levels of ionizing radiation and ultraviolet light, with measurements possible at the resolution of a single cell. These measurements will provide us, for the first time, quantification of DNA damaging events. This study will provide an instrumental platform from which numerous studies can be launched to explore the fundamental processes of DNA repair, the role of metabolic and exogenous sources of DNA damage, and possible preventative measures.

\section{Mission Relevance}

Quantification of DNA repair in cells damaged by low levels of ionizing radiation and ultraviolet light will lead to improved biological measurement and detection technologies in support of Lawrence Livermore missions in bioscience to improve human health and in national security. Understanding the effects of radiation on cells is a focus of DOE's lowdose radiation research interests. This study will also provide a platform for analyses of other carcinogenic environmental factors, such as chemical contaminants, contributing to LLNL's mission in environmental management.

\section{FY06 Accomplishments and Results}

In FY06, we measured the spontaneous turnover of DNA from very small numbers of cells (less than 10). We found that in our culturing conditions, a cell replaces approximately 5.3 million nucleotides, or $0.04 \%$ of its genetic material, every 24 hours. Such a high level of sensitivity should allow for detection of DNA repair associated with small amounts of DNA damage, making the procedure an extremely sensitive method for detecting repair associated with very low doses of DNA-damaging agents such as environmental or chemotherapeutic chemicals. 


\title{
Developing a New Accelerator Mass Spectrometry Assay for Quantitation of Platinum-DNA Adducts for Response to Platinum-Based Chemotherapy
}

\author{
Sang Soo Hah 06-LW-023
}

\begin{abstract}
Platinum-based drugs, which kill cancer cells through toxic DNA damage, are the most successful class of compounds for cancer treatment. However, many patients are unresponsive to treatment or acquire drug resistance. Using carbon-14-labeled carboplatin (a platinum-based anticancer drug) and accelerator mass spectrometry (AMS), the most sensitive method for studying long-lived isotopes, we will measure platinum-DNA adducts in cultured cancer cells, mice, and humans. This real-time pharmacokinetics study, conducted in collaboration with researchers from the University of California Davis Cancer Center, aims to develop an assay for determining which bladder cancer patients will benefit from carboplatin treatment.
\end{abstract}

This project, using AMS detection, will develop robust assays for quantitation studies on platinum-DNA adduct formation and repair. The unique analytical method developed is expected to provide the scientific proof-of-principle framework for AMS techniques that will proceed through increasing levels of complexity, to be broadly applicable to the study of other anticancer drugs. Using AMS to determine the pharmacokinetics and correlating the results to individual outcome will lead to better carboplatin treatment for bladder cancer patients. Applications of these techniques include discovery of pharmacokinetics, drug delivery, or mechanism elucidation that can eventually be applied to human clinical and epidemiological studies and to chemotherapy in humans.

\section{Mission Relevance}

The ability to track pharmokinetics at exceedingly low isotopic doses will directly contribute to Lawrence Livermore's mission in biosciences to improve human health and has potential applications in biothreat detection for the Laboratory's missions in national and homeland security.

\section{FY06 Accomplishments and Results}

In FY06, we developed an AMS-based, drug-induced DNA damage assay using carboplatin. The resulting assay enabled us to better understand both the in vitro kinetics of platinumDNA adduct formation and drug uptake, as well as DNA binding in T24 human bladder cancer cells. Importantly, the rate of carboplatin-DNA adduct formation was observed to be about 100 -fold slower than that reported for the more potent analog cisplatin, which explains the lower toxicity of carboplatin. The lowest concentration of radiocarbon in DNA of T24 cells was measured to be $\sim 0.1 \mathrm{amol} / \mu \mathrm{g}$ DNA, which is about 500 times more sensitive than limits of other platinum-DNA detection methods. In addition, we have filed a patent application on real-time individualized therapy evaluation. 


\title{
Proposed Work for FY07
}

In FY07, we will expand our cell-based studies of drug uptake and repair to include mice and then humans. A series of human cells with different DNA repair capacities will be exposed to carbon-14-labeled carboplatin under various conditions. Mice will be used to develop the assay for in vivo use. These efforts will enable us to correlate the adduct levels with cells' sensitivity to the platinum-based drugs and determine the correct dose of carboplatin for humans. After demonstrating the AMS assay in mice, we will use the assay on five human bladder cancer patients via our collaborations with the Davis Cancer Center.

\section{Publications}

Hah, S. S., and P. T. Henderson, 2006. In vitro kinetics of carboplatin-DNA binding in genomic DNA and bladder cancer cells as determined by accelerator mass spectrometry. 232th National Mtg. American Chemical Society, San Francisco, CA, Sept. 10-14, 2006. UCRL-POST-222801.

Hah, S. S., and P. T. Henderson, 2006. "Biomedical applications of accelerator mass spectrometry: kinetics of carboplatin-DNA binding in genomic DNA and bladder cancer cells." Environ. Mol. Mutagen. 47, 437. UCRL-ABS-219774.

Hah, S. S., et al., 2006. "Kinetics of carboplatin-DNA binding in genomic DNA and bladder cancer cells as determined by accelerator mass spectrometry." Chem. Res. Toxicol. 19, 622. UCRL-JRNL-217933.

\section{Analysis of the Mucin Membrane Protein by Cryo-Electron Microscopy and Computational Image Processing}

Joseph B. Pesavento 06-LW-064

\begin{abstract}
The membrane protein mucin 1 is involved in some types of breast and prostate cancer. Specifically, mucin 1 is linked to the survival of aberrant, precancerous cell types. However, like most membrane proteins, mucin 1 is difficult to prepare in a solubilized form and has not been amenable to study by $\mathrm{x}$-ray crystallography or nuclear magnetic resonance (NMR). We are in the process of expressing, purifying, imaging, and structurally analyzing the membrane-associated portions of mucin 1 using a combination of cryo-electron microscopy (cryo-EM) and computational image processing. Our results will provide a better understanding of both the normal and abnormal functions of mucin 1 , which should lead to improvements in diagnostics and therapeutics for cancer treatment. Imaging is being conducted at UC Davis and LLNL, and image processing on supercomputers at LLNL.
\end{abstract}

We expect our results to be twofold. First, we intend to deliver a new method for producing membrane proteins under conditions that facilitate their incorporation into lipid bilayers and imaging by cryo-EM, followed by three-dimensional reconstruction and visualization. A paper describing these results (specifically the cell-localization behavior 
of the protein and cell-surface characteristics) will be submitted for publication in a peerreviewed journal. Second, our work will result in a parallelized code and image-processing workflow for large image sets of cryo-EM data, protein, and macromolecular-complex data processing and structural reconstructions. This project will help determine the biochemical and structural basis for mucin 1's cancer-causing properties.

\title{
Mission Relevance
}

Our project contributes to the Laboratory's national security, homeland security, and biosciences missions. Technology from this research will be used to analyze the structures of microbial pathogens such as Yersinia pestis and Bacillus anthracis, as well as viruses such as coronavirus (SARS), vaccinia, and hemorrhagic fever. In addition, energy security research will benefit by the improvements to cryo-EM capabilities.

\section{FY06 Accomplishments and Results}

In FY06, we (1) produced and purified mucin 1 in collaboration with a noted mucin 1 and cancer researcher, (2) began preliminary imaging of mucin 1 by cryo-EM, ( 3 ) adapted cryo-EM image-processing software and tools to LLNL's infrastructure, and (4) submitted a record of invention for microscale forceps for electron microscopy.

\section{Proposed Work for FY07}

In FY07, we will (1) continue with our production and characterization of mucin 1, with focus shifting from the tandem-delete-region form of the protein to production of the full-length, glycosylated form using both cell culture and in vitro translation; (2) transition the collection of mucin 1 data sets from UC Davis to LLNL; (3) complete the development of novel cryo-EM image-processing algorithms and compile and test them to enable the image processing of large data sets on a regular basis; and (4) publish our mucin 1 structure and image-processing methodologies.

\section{Single-Cell Chromatography}

\author{
Mark G. Knize 06-FS-007
}

\begin{abstract}
We propose to develop a unique capability for single-cell analysis: micro-thin-layer chromatography coupled to time-of-flight secondary-ion mass spectrometry (ToF-SIMS). To date, single-cell analyses have been restricted to sensitive but low-information detection techniques that are unable to detect unknowns or characterize pathways. With
\end{abstract}


this new technique we will be able to detect, identify, and characterize components in single cells that will allow us to analyze unculturable organisms and detect rare events of early infection and internal chemical exposure of single cells. We will use Lawrence Livermore expertise in microfabrication and microfluidic separations to couple single-cell separation with the sensitivity of ToF-SIMS to develop a tag-free method of analysis.

We will couple our expertise in microfluidic separations with that in imaging mass spectrometry to greatly improve analysis of single biological cells. Successfully developing this capability will provide Lawrence Livermore with a unique tool to perform rapid (within a few minutes), detailed studies of individual cells. This analysis technique will enable the detection of host response to infection, cell response to radiation exposure, or quantify environmental exposure at the single-cell level. We will develop a practical, new forensic technique that does not rely on amplification for sensitive detection. This could lead to commercial applications in pathogen characterization and personalized medicine.

\section{Mission Relevance}

The development of single-cell chromatography capability is well-aligned with the LLNL mission to leverage engineering and physical science expertise to biochemical research directed at understanding the causes and mechanisms of ill health, developing biodefense capabilities for homeland security, improving disease prevention, and lowering healthcare costs. Specifically, it is relevant to applications in host-pathogen interactions, forensics, and environmental health sciences.

\section{FY06 Accomplishments and Results}

In FY06, we (1) fabricated thin-layer, electrically-conductive chromatographic substrates using the polymerization of methyltrimethoxy siloxane (the alternative silicon micropillar and carbon nanotube surfaces we explored also showed promise, but were more fragile); (2) established the technology to deposit mammalian cells and dissolve those cells in nanoliter-sized droplets of solvent mixtures; and (3) performed imaging mass spectrometry (ToF-SIMS) of target chemicals such as phenylalanine and caffeine on the synthesized chromatographic substrates. 
Laboratory Directed Research and Development

\section{Chemistry}




\title{
XChem
}

Ricky Chau

04-ERD-024

\begin{abstract}
This project addresses the fundamental nature of dynamic chemical processes at high temperatures, very high pressures, and short timescales (10-1000 ps) during the passage of a strong shock wave. By exploiting the properties of a steady shock wave produced by a gas gun or laser, we can enhance our spatial and temporal resolution using optical spectroscopy to probe the development of fast chemical processes in a simple molecular fluid. Benzene, cyclohexane, and nitrobenzene will be the prototype systems for this study, which will focus on understanding several fundamental processes such as carbon formation, dissociation, and the formation of intermediate species. Experimental data from this project will be compared to computational results.
\end{abstract}

The success of this project will determine fundamental properties of chemical reactions under extreme conditions of temperature and pressure. One result will be experimental data on kinetics and rates of chemical reaction at the subnanosecond timescale. In addition to improving chemical models, the data will impact many areas of science, including planetary science and energetic materials, and will enable the Laboratory to help fill substantive gaps in our knowledge of high-pressure chemistry.

\section{Mission Relevance}

Results from this project will help improve the many chemical codes used to study shorttimescale chemical processes involving energetic materials, which are essential to national security. Other applications of this research include the verified in situ destruction of chemical or biological weapons of mass destruction.

\section{FY06 Accomplishments and Results}

Our major accomplishment in FY06 was measurement of the dissociation process of cyclohexane using double-pass absorption. Unlike our previous experiments on benzene, cyclohexane required significantly higher shock pressures for dissociation. Over a wide range of pressures, an incubation time of over 100 ns was observed for the scattering of the incident light by dissociated carbon. In addition, we observed a systematic wavelength dependence of the absorption and an evolution of particle-size distribution. Overall, this project has shown that relevant chemical processes and kinetics can be probed experimentally over a wide range of timescales. 


\title{
Bioforensics: Characterization of Biological Weapons Agents by NanoSIMS
}

\author{
Peter K. Weber 04-ERD-039
}

\begin{abstract}
The objective of this project is to identify elemental and isotopic markers in spore material that can be used for attribution of biological weapons, including markers that allow identification of the production method, location, and date. We are developing the capability to analyze individual bioweapon particles for trace elements and isotopic signatures, building a library of elemental and isotopic fingerprints, and relating these signatures to specific processes, locations, and dates of production or release using the unique capabilities of the nanoscale secondary ion mass spectrometer (NanoSIMS). The NanoSIMS is ideally suited to characterization of biological weapons agents because it combines high spatial resolution with high sensitivity. In addition, NanoSIMS allows us to work with very small amounts of evidentiary material, analyze spores separately from weapon-manufacturing materials and environmental contaminants, and make characterizations of individual spores at the substructure level.
\end{abstract}

If successful, the project will establish the basis for using elemental and isotopic signatures to aid law enforcement and national security entities in their attribution efforts. We will establish the methodology for making these assessments of the method, region, and date of spore production.

\section{Mission Relevance}

This research project will contribute to the LLNL's national and homeland security mission by addressing the need for technologies to quickly detect, identify, and mitigate the use of chemical and biological threat agents.

\section{FY06 Accomplishments and Results}

In FY06, we developed the capability to perform isotopic analyses at the individual spore level and demonstrated that spore samples can be distinguished based on carbon isotopes, which helps in understanding the reproducibility of elemental signatures in spore preparations. Following up on our diffusion-rate experiments, we performed depth profiles at the individual spore level at high lateral and depth resolution to reveal elemental gradients with high efficiency. In addition to using this depth-profile method to study microstructural signatures, we used it to demonstrate that ions can rapidly diffuse into the core of spores in hydrated conditions. These results show that post-sporulation elemental uptake and loss-including that during sample preparation-can be important controls on elemental composition and gradients in spores, which help to address the technical issues of precise isotopic measurements in small samples. 


\section{Publications}

Brewer, L. N., et al., 2006. Microanalytical methods for bio-forensics investigations. Microscopy and Microanalysis, Chicago, IL, Aug. 2, 2006. UCRL-CONF-219020.

Ghosal, S., et al. 2006. Analysis of bacterial spore permeability to water and ions using nano-secondary ion mass spectrometry (NanoSIMS). 231st American Chemical Society National Mtg., Atlanta, GA, Mar. 26-30, 2006. UCRL-CONF-217312.

Ghosal, S., et al., 2006. Analysis of bacterial spore permeability to water and ions using nano-secondary ion mass spectrometry (NanoSIMS). 232nd American Chemical Society National Mtg., San Francisco, CA, Sept. 10-14, 2006. UCRL-ABS-220927.

Ghosal, S., et al., 2006. Characterization of bacterial spores using nano-secondary ion mass spectrometry (NanoSIMS). Gordon Research Conf. Chemistry at Interfaces, Biddeford, ME, July 9-14, 2006. UCRL-ABS-222301.

Weber, P. K., et al., 2006. Chemical characterization of bacterial spores by NanoSIMS. American Society of Clinical Oncology Microbial Bioforensics Workshop, Defense Threat Reduction Agency, Ft. Belvoir, VA, June 21, 2006. UCRL-CONF-222166.

Weber, P. K., et al., 2006. Chemical imaging of biological materials by NanoSIMS using isotopic and elemental labels. 19th Ann. SIMS Workshop, Rancho Mirage, CA, May 19, 2006. UCRL-PROC-220500.

Weber, P. K., et al., 2006. High resolution trace element and isotopic imaging of microbial systems by NanoSIMS. American Geophysical Union Fall Mtg., San Francisco, CA, Dec. 5-9, 2005. UCRL-CONF-215311.

\section{Ionization Chemistry of High-Temperature Molecular Fluids}

Laurence E. Fried 04-ERD-069

\section{Abstract}

The purpose of this project is to investigate the phase diagram of simple polar molecular fluids (water and ammonia) and mixtures under conditions of extreme pressure (>10 GPa) and temperature $(>1000 \mathrm{~K})$. Under extreme conditions, the neutral molecular form of matter transforms to a phase dominated by ions, the boundaries of which are unknown. We will perform tightly coupled modeling and experiments to determine the phase diagram of simple fluids in this region. Proposed novel "superionic" lattice states involving mobile hydrogen atoms will be investigated. Acid-base chemistry under extreme conditions will be studied in a diamond anvil cell for the first time, and the chemistry of synthetic planetary interiors will be addressed through molecular mixtures. 
Our proposed research will lead to the first understanding of ionization and acid-base chemistry under extreme conditions. We have already achieved experimental and computational results that indicate the existence of nonmolecular phases of water over $50 \mathrm{GPa}$, and that qualitatively new superionic phases may be found. By mixing water and ammonia under extreme pressure and temperature, we will understand how fundamental chemical notions such as acid-base chemistry are modified in extreme environments. This project increases our understanding of planetary interiors and will guide the modeling of high-explosive detonations. The results are of great interest to researchers in high-pressure physics, and have already generated articles in high-visibility journals such as Nature.

\section{Mission Relevance}

The results of this investigation will be coordinated with Advanced Simulation and Computing program efforts in high-explosive modeling. Future developments in the Cheetah and CHEQ high-explosive detonation codes will be based on new knowledge of high-pressure chemistry generated by this project. The Cheetah and CHEQ codes support the Laboratory's stockpile stewardship mission and extend scientific understanding in support of the basic science mission.

\section{FY06 Accomplishments and Results}

In FY06, simulations were performed on the simple acid hydrogen fluoride, and we determined it had a superionic phase at experimentally accessible conditions (30 GPa, $900 \mathrm{~K}$ ). We also performed $\mathrm{x}$-ray scattering experiments to determine the structure of liquid water at extreme conditions. The results showed qualitative agreement with simulation, but substantial differences were also found. Overall, this project succeeded in identifying, through combined experiment and simulation, the existence of a nonmolecular superionic phase of water. Our work has been published in prestigious, peer-reviewed journals such as Physical Review Letters.

\section{Publications}

Fried, L. E., et al., 2005. Chemistry of $\mathrm{H}_{2} \mathrm{O}$ and $\mathrm{HF}$ under extreme conditions. Shock Compression of Condensed Matter, Baltimore, MD, July 31-Aug. 5, 2005.

UCRL-CONF-217385.

Fried, L. E., et al., 2006. Non-molecular phases of $\mathrm{H}_{2} \mathrm{O}$ and $\mathrm{HF}$ under detonation-like conditions. 13th Intl. Detonation Symp., Norfolk, VA, July 23-28, 2006.

UCRL-PROC-222768.

Goldman, N., and L. E. Fried, 2006. "First-principles simulation of a superionic phase of hydrogen fluoride (HF) at high pressures and temperatures." J. Chem. Phys. 125, 044501. UCRL-JRNL-220577. 


\title{
New Fragment Separation Technology for Superheavy Element Research
}

\author{
Dawn A. Shaughnessy 04-ERD-085
}

\begin{abstract}
We propose to investigate the western edge of the "island of stability"-an unexplored high-mass region of the periodic table where elements are predicted to have particularly stable "magic numbers" of protons and neutrons-to determine whether or not the closed proton shell is located at 114 protons. Probing the influence of the closed proton shell has profound implications for nuclear physics models. We will accomplish this by (1) fabricating a thick plutonium ceramic target that will be bombarded with calcium-48 to produce element-114 isotopes, which will be separated in the Mass Analyzer for Super Heavy Atoms (MASHA) in Dubna, Russia to identify the atomic mass of the element-114 isotopes and (2) irradiating californium-249; curium-245, 248; and americium-243 targets to produce elements 118,116 , and 115 , which will further define the edge of the island of stability.
\end{abstract}

If these experiments are successful, we will discover a new element (element 118), better establish the decay properties of the isotopes of elements 115 and 113, and gather additional experimental evidence to help determine whether the island of stability is centered at 114 protons or at a higher atomic number. We also expect that the results of these experiments will extend and improve theoretical models used to calculate the decay properties and nuclear shapes of the heaviest elements. As part of these studies, we will perform chemical separations on the longer-lived decay daughters of elements 115 and 114 , which in turn will give us insight into the chemical properties and behavior of the heavy elements compared to their lighter homologues in the periodic table.

\section{Mission Relevance}

Efforts to synthesize new elements and measure their properties support the national security mission by furthering competencies in nuclear chemistry and radiochemistry, which are relevant to assessing nuclear device performance and countering proliferation activities involving nuclear materials. This project also supports the environmental management mission by producing technology for the safe disposal of radioactive waste.

\section{FY06 Accomplishments and Results}

During FY06, we: (1) continued work on the synthesis of ceramic targets at MASHA for producing a plutonium-244 target for production of element 114. As part of this effort, we investigated new synthetic methods and conducted tests on materials; (2) investigated the possibility of in-beam tests of these targets at several facilities; ( 3 ) developed a new chemical separation for element $105 ;(4)$ chemically isolated and identified the element115 decay daughter; (5) found evidence that element 105 behaves more like its homolog 
tantalum rather than niobium; (6) continued irradiation of americium-243, curium-245, and californium-249, which concluded with the submission of a paper describing the production of element 118; and (7) continued research on MASHA with our Russian collaborators.

\section{Proposed Work for FY07}

In FY07, we will (1) continue target development, which will include possible in-beam tests; (2) further develop element-105 chemical separations for an experiment in Dubna; (3) begin development of a chemical separation for element 104, which will help identify the decay daughter of element $114 ;(4)$ begin construction of an automated chemistry apparatus that can be used for element-104 chemistry and, depending on the schedule in Dubna, deploy this system by year's end; and (5) continue production runs with the neptunium-237 + calcium-48 and iron-58 + plutonium-244 reactions to synthesize elements 113 and 120 , conducting additional bombardments based on the results obtained from experiments in FY06.

\section{Publications}

Oganessian, Y. T., et al., 2006. "Synthesis of the isotopes of elements 118 and 116 in the Cf-249 and Cm-245 + Ca-48 fusion reactions." Phys. Rev. C. 74, 044602.

UCRL-JRNL-220958.

Shaughnessy, D. A., et al., 2006. Ceramic target development for heavy isotope production and the rare isotope accelerator. European Materials Research Society Mtg., Nice, France, May 29, 2006. UCRL-ABS-225155.

Shaughnessy, D. A., et al., 2005. Chemical identification of dubnium as a decay product of element 115. Pacifichem, Honolulu, HI, Dec. 15-20, 2005. UCRL-CONF-217831.

Shaughnessy, D. A., et al., 2006. Radiochemical isolation of dubnium as the decay product of element 115. American Chemical Society National Mtg., San Francisco, CA, Sept. 10-14, 2006. UCRL-ABS-221534.

Stoyer, N. J., et al., 2006. "Chemical identification of a long-lived isotope of dubnium, a descendant of element 115." Proc. 9th Intl. Conf. Nucleus Nucleus Collisions. UCRL-PROC-224777.

Stoyer, M. A., 2006. "Island ahoy!" Nature 442, 876. UCRL-JRNL-224404.

Stoyer, M. A., et al., 2006. Nuclear alchemy-the sorcery of synthesizing new chemical elements. American Chemical Society National Mtg., Atlanta, GA, Mar. 26-30, 2006. UCRL-ABS-218506.

Stoyer, M. A., 2006. The heaviest exotic nuclei in the laboratory-superheavy nuclei. 2006 Ann. Mtg. Division of Nuclear Physics of the American Physical Society, Nashville, TN, Oct. 25-28, 2006. UCRL-ABS-222665. 


\title{
Heterogeneous Processes at the Intersection of Chemistry and Biology
}

\author{
Christopher J. Mundy 05-ERD-021
}

\begin{abstract}
Phenomena in heterogeneous environments are important processes in fields ranging from chemistry, such as the fate of aerosolized chemical weapons, to atmospheric science, such as ozone destruction. However, no computational framework exists to address such phenomena. One of the difficulties in studying heterogeneous processes is that current empirical molecular-potential models are parameterized to reproduce only bulk liquid properties. We propose to investigate heterogeneous chemistry in aerosols and enzymes using terascale ab initio methods, which are well suited to providing an unbiased representation of the force field in mesoscale environments and also allow us to easily incorporate reactivity.
\end{abstract}

Using LLNL's terascale resources in conjunction with state-of-the-art software, we will conduct research on heterogeneous chemistry as applied to biological and atmospheric systems. Using these unique capabilities, we expect to solve significant problems for a broad set of scientific disciplines.

\section{Mission Relevance}

The computational suite developed in this project directly benefits the national security mission by contributing, for example, to increased understanding of the physical properties of organophosphates such as sarin and VX, which will lead to improved detection and ability to predict their fate and transport. Specifically, this technology can provide a model of sarin release at the city scale by incorporating our microscopic understanding of heterogeneous processes.

\section{FY06 Accomplishments and Results}

In FY06, we performed large-scale quantum mechanical/molecular mechanical (QM/MM) calculations of orotidine 5'-monophosphate (OMP) and 6'-hydorxyuridine-5'monophosphate (BMP) in an orotidine monophosphate decarboxylase (ODCase) system of 30,000 atoms to study enzymatic reactions. Within this scheme, we investigated reaction mechanisms leading to decarboxylation, such as a possible proton transfer to $\mathrm{O} 4$ and lysine attack on C4 leading to an iminium compound. For aerosols, we performed metadynamics calculations on multiple sizes of microsolvated clusters (ranging from a cluster of six waters up to bulk water) and compared mechanisms for acid dissociation. We also sought out structural moieties on large surface models to connect directly with cluster calculations. 


\title{
Proposed Work for FY07
}

In FY07, we will (1) continue our large-scale QM/MM calculations with OMP in ODCase; (2) map out the full free-energy surface of possible reaction mechanisms including hypothesized covalent intermediates, and deduce possible transition states; (3) perform umbrella- and path-sampling techniques on these transition states to properly deduce the reaction mechanism for decarboxylation; (4) validate the use of semi-empirical methods to reproduce reaction mechanisms obtained in FY06; and (5) use semi-empirical methods to perform the first in situ studies of the uptake of nitric acid by sea-salt aerosols.

\section{Publications}

Kuo, I. F. W., C. J. Mundy, and M. J. McGrath, 2006. "Time-dependent properties of liquid water: a comparison of Car-Parrinello and Born-Oppenheimer molecular dynamics simulations." J. Chem. Theor. Comput. 2, 1274. UCRL-JRNL-218411.

McGrath, M. J., et al., 2006. "Simulating fluid-phase equilibria of water from first principles." J. Phys. Chem. 110, 640. UCRL-JRNL-210218-DRAFT.

Mundy, C. J., and I. F. W. Kuo, 2006. "First-principles approaches to the structure and reactivity of atmospherically relevant aqueous interfaces." Chem. Rev. 106, 1282. UCRL-JRNL-212909.

\section{Avoiding Surprise: Countering Novel Chem-Bio-Warfare Agent Threats}

Bradley R. Hart

05-ERD-025

\begin{abstract}
Chemical synthesis and computational modeling, when combined, can aid in characterizing the physiological capabilities and properties of candidate compounds that could be designed by an adversary to be either incapacitating or lethal chemical and biological weapons (CBW) agents. From this information, detection and countermeasures strategies can be designed. We propose to define, develop, and implement a comprehensive scientific approach that will couple cutting-edge computational chemistry and new synthetic methods to enhance our understanding of the threats posed by the potential development and use of novel CBW agents. The results of this research are intended to cue U.S. defensive efforts by providing guidance for creating new detection and countermeasures programs.
\end{abstract}

\section{Mission Relevance}

This research is designed to address a serious national security concern and to demonstrate a state-of-the-art capability to the broader community of U.S. government 
agencies. Therefore, our work directly supports the Laboratory mission of enhancing national security and facilitating efforts to halt and reverse the proliferation of weapons of mass destruction.

\title{
FY06 Accomplishments and Results
}

In FY06, major milestones were met with the completion of homology models of two protein targets, as were molecular dynamics simulations of anesthetic binding. We also evaluated synthesis mechanisms and began to determine their affect on specific synthesis routes.

\section{Proposed Work for FY07}

For FY07, we plan to continue exploration of synthesis and computational modeling as methods for characterizing the physiological capabilities and properties of candidate compounds that could be designed by an adversary as either incapacitating or lethal CBW agents. This work will include performing molecular dynamics simulations of interactions between the homology models developed through this project and chemicals of interest. Specifically, we will attempt to determine regions of the protein that are likely to interact most strongly with these chemicals. The experimental portion of this project will transition from focusing on the mechanics of the required molecular transformations to the synthesis of the target compound(s). This will be followed by a complete chemical/ physical characterization of these targets to include key spectroscopic information and decomposition rates in various environments.

\section{Discovering the Folding Rules that Proteins Obey}

\author{
Olgica Bakajin \\ 05-ERD-078
}

\begin{abstract}
Protein folding is a fundamental cellular process: Proper folding is required for a protein to carry out its functions, while improper folding can be a source of disease. We propose to use a combination of simulations and experiments to significantly advance our understanding of the molecular mechanisms of protein folding. We will develop a robust microfluidic mixing device, conduct long-time simulations on supercomputers, and perform measurements on the systems that exhibit fast folding and fast hydrophobic collapse. Our results will be used to answer the following questions: Can traps and intermediate states be observed? Why are some molecules such fast folders? Are there multiple folding pathways, a few, or just one? And is folding hierarchical?

We expect to elucidate the mechanisms of protein folding through a combination of complementary experimental and simulation studies. In addition, the equipment and technology developed for this project will provide new capabilities that will be applicable to numerous projects at LLNL. This research will establish and strengthen collaborations between LLNL scientists and leading researchers in academia and industry. Because of the
\end{abstract}


fundamental nature of this project, it should result in publications in high-impact, peerreviewed journals.

\section{Mission Relevance}

By preparing the scientific basis for understanding and controlling protein function, this project is highly relevant to the Laboratory's mission in biodefense. The knowledge base created in this project also will support the Laboratory's mission in bioscience to improve human health.

\section{FY06 Accomplishments and Results}

In FY06, we reduced mixing time through shape optimization and performed measurements of folding kinetics using unlabeled proteins in the ultraviolet spectrum, and Forster resonance energy-transfer measurements of labeled proteins in the visible spectrum. These measurements revealed two separate, previously unresolved processes: a spectral shift that occurs within the mixing time and a fluorescence decay occurring between 100 and $300 \mu \mathrm{s}$. We attribute the first process to hydrophobic collapse and the second process to formation of the first tertiary contacts. We also performed a simulation study on a small, 40-amino-acid, two-helix bundle protein (1BBL).

\section{Proposed Work for FY07}

In FY07, we propose to (1) further decrease mixing time with a combination of shape optimization and feature-size reduction; (2) fully characterize mixers, fabricate them in fused silica and silicon, and make them available for experiments; (3) develop a capability for doing mixing experiments at elevated temperatures; (4) evaluate the feasibility of fast cooling through mixing; (5) fabricate and test prototypes; (6) perform simulations from experimentally relevant starting conformations; (7) focus on a fast-folding variant of the DNA binding domain from the lambda repressor that can also be addressed experimentally; and (8) perform measurements with sub-microsecond time resolution on the lambda repressor.

\section{Publications}

Bakajin, E., et al., 2006. "Materials aspects in micro- and nanofluidic systems for DNA analysis and protein folding." MRS Bulletin 31, 108. UCRL-JRNL-217803.

Hertzog, D. E., et al., 2006. "Optimization of a fast microfluidic mixer for studying protein folding kinetics." Anal. Chem. 78, 4299. UCRL-JRNL-217805.

Kane, A. S., et al., 2006. Microfluidic mixers for the investigation of protein folding using synchrotron radiation circular dichroism (SRCD) spectroscopy. Solid-State Sensors, Actuators, and Microsystems Workshop, Hilton Head, SC, June 4-8, 2006.

UCRL-PROC-220084. 


\title{
Conversion of Plutonium and Enriched Uranium
}

\author{
Thomas W. Trelenberg 06-ERD-012
}

\begin{abstract}
This project seeks to determine the mechanisms governing hydride and oxide conversions of uranium and plutonium. The unpredictable nature of these conversion processes raises long-term storage and retrieval concerns for these materials and highlights a lack of understanding about how impurities serve to catalyze the reactions and what effect they might have on archived scientific samples. Both macroscopic (coupon tests) and microscopic surface science experiments via photoemission measurements will be used to characterize the reaction rates with and without catalysts and mechanisms that convert plutonium and uranium into their respective hydrides and oxides. Computer simulations using relativistic quantum models guide the experimental program. Experimental data will, in turn, help verify the modeling results.
\end{abstract}

The integration of our experimental and theoretical findings will lead to a predictive capability for these reactions and will be used to define the mechanisms governing these conversions, providing insight into their proper application to plutonium and uranium storage and retrieval.

\section{Mission Relevance}

Actinide compounds (such as hydrides or oxides) are of interest for the storage, recovery, and retrieval of nuclear materials in support of the Laboratory's national security missions in stockpile stewardship and nonproliferation.

\section{FY06 Accomplishments and Results}

We (1) computed the electronic structural and spectroscopic properties for the low-lying electronic states of $\mathrm{PuH}_{n}(n=2-4)$, providing insight into how these compounds become autocatalytic to further hydriding; (2) made significant progress toward operation of the surface science system with actinide samples after determining the facility and instrument requirements for actinide introduction and removal; (3) conducted coupon tests to examine the corrosion of uranium and plutonium under various conditions, highlighting the importance of system cleanliness to ensure experimental repeatability; and (4) constructed a separate vacuum system to examine alternative methods of achieving acceptable contaminant levels prior to experimentation.

\section{Proposed Work for FY07}

In the theoretical component, we will calculate the potential energy surfaces of plutonium clusters interacting with $\mathrm{H}_{2}$ as a function of $\mathrm{H}_{2}$ orientation, approach, and distance; 
calculate the hydride activation energy barrier; and evaluate the effects of selected elemental impurities on the activation barrier, possibly suggesting catalyzing agents. Finally, with modifications to the coupon instruments and using techniques learned from the contaminate removal system, we will begin advanced experiments with catalysts and introduce actinides (beginning with uranium) into the surface science instrument.

\title{
Publications
}

Balasubramanian, K., et al., 2006. Atomistic level relativistic quantum modelling of plutonium hydriding. Plutonium Futures: The Science 2006, Pacific Grove, CA, July 9-13, 2006. UCRL-ABS-218641.

\section{Long-Time-Scale Shock Dynamics of Reactive Materials}

\author{
Nir Goldman 206-ERD-037
}

\begin{abstract}
We propose to study the long-time-scale effects of shocks on highly reactive materials using a novel shock dynamics technique, the multiscale shock method (MSSM), implemented in an ab initio molecular dynamics (MD) code. Until recently, existing techniques for quantum simulations of shocked materials have been prohibitively expensive. The combination of MSSM with an ab initio MD code will elucidate answers to key questions regarding materials decomposition. We propose to conduct shock simulations of simple liquids such as water, and simulate shocked astrochemical mixtures of prebiotic molecules that are found in comets and other celestial bodies.

Our study will provide the first use of a state-of-the art quantum mechanical simulations code to study shocked, highly reactive materials. If successful, these simulations will allow us to determine the exact kinetic and thermodynamic nature of chemical reactivity at high temperature and pressure. From them we will gain important insight into how simple molecular systems are influenced by rapid compression and how this pertains to geochemical and planetary processes. We expect to publish our results in peer-reviewed journals.
\end{abstract}

\section{Mission Relevance}

Knowledge of highly reactive materials decomposition under conditions of high temperature and pressure is essential for a complete understanding of liquids in the interiors of Earth and large planets and is central to Lawrence Livermore's stockpile stewardship mission. The quantum simulations algorithms and codes developed in this project will be applicable to several national security missions. In addition, this high-profile scientific research supports LLNL's mission in breakthroughs in fundamental science and technology, and will attract new talent to the Laboratory. 


\title{
FY06 Accomplishments and Results
}

After a midyear start, we (1) interfaced the MSSM shock wave MD code with the CP2K and CPMD codes; (2) conducted tests on smaller, previously published model systems, including silicon; and (3) completed quantum simulations of the shock Hugoniot of water. These efforts have given us a unique capability to conduct research in a fundamentally new regime. We have shown that density functional theory molecular dynamics results compare extremely well to experiments on the water shock Hugoniot. Our results represent the strongest confirmation of the accuracy of the density functional theory at high pressure and temperature that we know of, to date. We have prepared two manuscripts on our water simulations.

\section{Proposed Work for FY07}

In FY07, we will develop metadynamic collective variables for use in the shock ensemble. Initially, we will conduct simulations of the oxygen-hydrogen bond dissociation in shocked water. We will also investigate solid-solid phase transitions, such as those from ice VIII to ice VII or from ice VII to superionic water. Our results will help determine the phase diagram of water beyond the conditions currently known. These results are vital for equation-of-state models for planetary science as well as energetic materials. We will also determine the ab initio parameters required for our simulations. This work will lay the foundations for studying a variety of other shocked materials.

\section{Biologically Driven Fabrication of Complex Nanostructures at Nanoscale Chemical Templates}

Sung-Wook Chung 06-LW-051

\begin{abstract}
The ability to deposit biomolecules such as RNA onto specific sites or into ordered arrays would facilitate many challenges in biology and nanotechnology, including biologically driven materials organization, determination of protein structures, and biological sensing. Perhaps most intriguing is the idea of combining biomolecules with inorganic nanostructures to form hierarchical multicomponent biological-inorganic hybrids. This project proposes to use nanoscale templated assembly of biomolecules to fabricate hybrid nanostructures and understand the physical forces driving nanostructure formation. Scanned probe nanolithography (SPN) will be used to create patterned structures as templates for organizing biomolecules. Initial experiments will pattern RNA catalysts on gold surfaces and study formation of palladium nanoparticles on these patterned surfaces.

Multicomponent biological-inorganic hybrid nanostructures are expected to result from this project, along with a better understanding of the physical forces at the bioinorganic interface that influence nanostructure formation. Leveraging the unique materialssynthesis properties of biomolecules, such as these RNA catalysts with controlled two- and
\end{abstract}


three-dimensional (2D and 3D) organization of biomolecules on pre-patterned nanoscale templates, is expected to lead to entirely new inorganic nanostructures of unique size, shape, and morphology. These unique characteristics are likely to result in novel properties that conventional, solution-based synthetic approaches cannot produce.

\section{Mission Relevance}

As a means for fabricating arrays of multicomponent hybrid nanomaterials, the biotemplated synthesis technology developed in this project has potential applications in novel thin films and materials for stockpile stewardship experiments on future fusion-class lasers. The combination of small size, high density, and biological integration could also enable high-sensitivity, high-selectivity pathogen detection for counterproliferation and counterterrorism missions. Finally, this technology has the potential for use in repairing defects or broken metallic electrodes in the circuits of custom electronic devices used in military and space applications.

\section{FY06 Accomplishments and Results}

In FY06, we (1) synthesized a library of chemo-selective linkers and newly designed RNA catalysts with a functional handle to recognize the linkers; (2) demonstrated the fabrication, by SPN, of $1 \mathrm{D}$ and 2D nanoscale template patterns on gold surfaces using our newly developed linker molecules; (3) performed preliminary experiments on the catalytic reactivity of two RNA catalysts, Pd017 and Pd034 PDase, which were shown to template the formation of palladium nanocrystals of hexagonal and cubic structure in solution, respectively; and (4) began investigation of morphology, structure, and mechanism of RNA-mediated palladium nanostructure formation.

\section{Proposed Work for FY07}

In FY07, we plan to (1) measure the structural and morphological evolution and kinetics of RNA-catalyzed, metallic-nanoparticle formation via in situ solution-imaging experiments; (2) characterize the physical interaction of the RNA-inorganic nanoparticle surface using (dynamic) chemical force microscopy; and (3) fabricate an array of metallic nanoparticles or nanocircuitry via RNA-catalyzed metallic-nanoparticle formation.

\section{Publications}

Chung, S.-W., et al., 2006. Growth of metal nanostructures on templates of RNA-aptamer catalysts formed by scanned probe nanolithography. American Chemical Society Annual Fall Mtg., San Francisco, CA, Sept. 10-14, 2006. UCRL-PRES-224364.

Chung, S.-W., et al., 2006. Growth of metal nanostructures on templates of RNA-aptamer catalysts formed by scanned probe nanolithography. The Materials Research Society Annual Spring Mtg., San Francisco, CA, Apr. 12-21, 2006. UCRL-ABS-220613. 
Laboratory Directed Research and Development

\section{Earth and Space Sciences}




\title{
Nonaqueous-Phase Liquid Dissolution in Porous Media: Multiscale Effects of Dissolution Kinetics on Cleanup Time
}

Walt W. Mcnab

\begin{abstract}
The goal of this research is to identify and quantify, in unprecedented detail, those factors controlling the rate at which dense, nonaqueous-phase liquid (DNAPL) contaminants dissolve into contaminant plumes in groundwater from source areas. This information is critical to understanding and predicting contaminant flow and transport and the efficacy of cleanup efforts over long periods. To achieve a quantitative understanding of the dissolution, migration, and remediation of DNAPL in the subsurface, the research effort will entail experimental and numerical investigations of the multiphase physics of the dissolution processes of DNAPL at the microscopic scale (i.e., involving solution of the Navier-Stokes equations).
\end{abstract}

By directly addressing DNAPL dissolution scaled up from pore-scale processes to field-scale models, this project will yield an improved understanding of the behavior of chlorinated solvent groundwater plumes in terms of plume longevity, morphology, and limitations on the efficacy of certain remediation technologies. On a practical level, this understanding can be used to better formulate plume-management strategies. On a fundamental level, the modeling and experimental techniques developed through this study will advance the understanding of contaminant hydrology processes on the microscale.

\section{Mission Relevance}

This project leverages environmental-restoration and groundwater-modeling skills at LLNL to improve the understanding of the behavior of groundwater contaminants in source areas. If successful, this project will have a direct effect on cleanup efforts at many sites across the DOE complex, in support of DOE's environmental-management mission.

\section{FY06 Accomplishments and Results}

Much of the research effort in FY06 focused on numerical (parallelized) evaluation of DNAPL dissolution, with capabilities extended to include discrete fractures and dual continuum porous/fracture systems. A nested correlation function for grain size and stochastic conditional simulations was implemented, enabling generation of realistic synthetic porous/fractured media. The experimental component of the study yielded highresolution images of the interaction of DNAPL ganglia with a commonly used oxidizing agent (potassium permanganate), which resulted in localized mineral precipitation reactions that altered the porous media structure. Overall, this project has provided an unprecedented data set and accompanying modeling tools that will aid management of the widespread environmental challenges posed by plumes of DNAPL-contaminated groundwater. 


\title{
Publications
}

McNab, W. W., S. M. Ezzedine, and R. L. Detwiler, 2005. Quantifying an intrinsic mass transfer rate for TCE dissolution via pore-scale experiments and simulations. DNAPL Source Zone Characterization and Remediation Symp., San Francisco, CA, Dec. 7-8, 2005. UCRL-PRES-217179.

\section{Magnetic Dynamos and Stars}

\author{
Peter P. Eggleton 04-ERD-027
}

\begin{abstract}
This project will focus on upgrading Djehuty, a code that models stars in three dimensions (3D), by incorporating magnetohydrodynamics (MHD). Djehuty already includes an accurate equation of state, radiative heat transport, a full network of nuclear reactions, self-consistent gravity in the spherical approximation, Lagrangian hydrodynamics, and an algorithm for adjusting severely distorted meshes. We will add the magnetic-force term to the hydrodynamics and the induction equation in the frozen-in approximation, enabling the code to model a wide range of inherently 3D problems currently beyond computational possibility, including dynamo activity in stars and planets and ultimately the Sun's wellknown, but poorly understood, magnetic cycle.
\end{abstract}

By end of this project, we expect to produce a code that incorporates MHD and has improved abilities to model a broad class of astrophysical phenomena. This code will be the first to tackle and understand the (1) generation of huge but transient ( 1 to 3 year) star spots observed on some red giant stars, (2) similar shorter-time scale phenomena in red dwarf stars, (3) magnetic behavior in gaseous planets such as Jupiter and many recently discovered extrasolar planets, (4) energy transport in contact binaries comprising two red-dwarf stars, and (5) the solar cycle, which strongly impacts Earth and Earthorbiting satellites. These significant scientific advances will enhance Lawrence Livermore's role in the astrophysical community, help recruit new talent to the Laboratory, and attract new collaborations with researchers at the University of California.

\section{Mission Relevance}

Because the thermonuclear phenomena found in stars are similar to those that occur in man-made nuclear explosions, this project will benefit numerical simulation and fundamental research used in stockpile assessment and in thermonuclear experiments at future, large fusion-class lasers, in support of the national security mission. Our results will also benefit atmospheric modeling, in support of the environmental management mission, and the exploration and use of space, which will benefit LLNL missions in national security and fundamental science. 


\title{
FY06 Accomplishments and Results
}

In FY06, we simulated a helium flash with our updated Djehuty code, and noticed an entirely unexpected phenomenon, which we term $\mu$ mixing. We continued to investigate rotation, but switched our main attention from magnetic fields to this new phenomenon, which occurs in normal red giants well before the helium flash, and has the effect of causing turbulent mixing over a much more significant part of the star than has been established previously. In particular, it lowers the abundance of helium-3 in the surface layers and increases the abundance of carbon-13. Observations have established that the helium-3 abundance cannot be as large as earlier models predicted, and carbon-13 is often enhanced above the predictions of earlier models. Thus, our mixing model appears to explain two phenomena that have puzzled astrophysicists for 20 to 30 years, and we have reported the results in high-profile, peer-reviewed journals.

\section{Publications}

Dearborn, D. S. P., J. C. Lattanzio, and P. P. Eggleton, 2006. "Three-dimensional numerical experimentation on the core helium flash of low-mass red giants" Astrophys. J. 639(1), 405. UCRL-JRNL-213547.

Eggleton, P. P., D. S. P. Dearborn, and J. Lattanzio, 2006. "Deep mixing of ${ }^{3} \mathrm{He}$ : reconciling Big Bang and stellar nucleosynthesis." Science 314, 1580. UCRL-JRNL-223274.

Lattanzio, J. C., D. S. Dearborn, and P. P. Eggleton, 2006. "Calculating stellar models in 3D: Djehuty and the core helium flash." Memor. Soc. Astronom. Ital. 77, 810.

UCRL-JRNL-224528.

\section{Dynamic Data-Driven Event Reconstruction for Atmospheric Releases}

Branko Kosovic 04-ERD-037

\begin{abstract}
Atmospheric releases of hazardous materials can have a powerful and rapid impact on large populations. For emergency response, forensic, and sensor network design needs, we are developing a flexible and robust data-driven, event-reconstruction capability and a supporting computational framework. Our approach couples data and predictive models with Bayesian inference and stochastic sampling to provide backward analyses to determine unknown source characteristics, optimal forward predictions for consequence assessment, and dynamic reduction in uncertainty as additional data become available.
\end{abstract}

Event reconstruction tools developed under this project will provide a unique capability for interpreting and responding to atmospheric releases. Such a capability addresses immediate critical needs for counterterrorism, consequence management, emergency response, attribution, and forensic analysis applications. The results of this project will enable optimal siting of sensors and facilitate uncertainty analyses that will be used in studies that assess vulnerability and protection of critical infrastructure. 


\section{Mission Relevance}

This project directly contributes to the Laboratory's homeland and national security mission by addressing a critical need for atmospheric release event-reconstruction tools that support the rapidly growing number of operational detection, warning, and incident characterization systems being developed and deployed by the Department of Homeland Security and Department of Energy. This capability will transform the way we respond to terrorist attacks, industrial accidents, and military engagements by reducing situationawareness uncertainties and facilitating informed decision making for effective response.

\section{FY06 Accomplishments and Results}

In FY06, we (1) implemented an operational Gaussian puff-dispersion model for simulation of urban atmospheric dispersion and tested it using data from the Oklahoma City Joint Urban 2003 experiment; (2) successfully demonstrated the continental-scale, Markov Chain Monte Carlo event-reconstruction capability using data from an accidental release of radioactive material at Algeciras, Spain in 1998; (3) developed an error quantification model for data, input parameters, and forward-model output error; and (4) developed and tested a computational framework including hybrid Markov Chain Monte Carlo-stochastic Monte Carlo algorithms on massively parallel platforms. For this project, we successfully developed, tested, and demonstrated a flexible and adaptable data-driven, eventreconstruction capability for atmospheric releases suitable for operational integration into an emergency response system. Applications of capabilities and techniques developed in this project are currently being considered for use by the National Atmospheric Release Advisory and Inter-Agency Modeling and Atmospheric Assessment centers.

\section{Publications}

Chow, T., B. Kosovic, and S. Chan, 2006. Source inversion for contaminant plume dispersion in urban environments using building-resolving simulations. 6th Symp. Urban Environment at 86th Ann. American Meteorological Society Mtg., Atlanta, GA, Jan. 30-Feb. 2, 2006. UCRL-CONF-216903.

Delle Monache, L., et al., 2006. Event reconstruction for atmospheric releases at the continental scale. A case study: May 1998, Algeciras, Spain. 10th George Mason University Conf. Atmospheric Transport and Dispersion Modeling, Fairfax, VA, Aug. 1-3, 2006. UCRL-PRES-223195.

Johannesson, G. K., et al., 2006. "Sequential Monte-Carlo framework for dynamic datadriven event reconstruction for atmospheric release." Proc. Nonlinear Statistical Signal Processing Workshop. UCRL-PROC-222915.

Lundquist, J. K., B. Kosovic, and R. Belles, 2006. Synthetic event reconstruction experiments for defining sensor network characteristics. UCRL-TR-217762.

Neuman, S., et al., 2006. Event reconstruction with the urban dispersion model. 6th Symp. Urban Environment at 86th Ann. American Meteorological Society Mtg., Atlanta, GA, Jan. 30-Feb. 2, 2006. UCRL-PROC-216842. 


\title{
Coupling Micromechanics and Reactive Fluid Flow in Fracture Networks
}

\author{
Russell L. Detwiler 04-ERD-046
}

\begin{abstract}
Flow of reactive fluids through fractured rock masses under changing stresses leads to potentially significant permeability changes that are difficult to predict with existing continuum models. Yet such permeability alterations are critical to a range of problems relating to energy and the environment. We are developing and applying coupled computational models that explicitly incorporate the small-scale physical mechanisms that control these processes in individual fractures. We then use this coupled small-scale model as a building block for a discrete fracture network model that explicitly incorporates the detailed processes within individual fractures, thus bridging a critical gap between our current knowledge of small-scale processes and field-scale behavior.
\end{abstract}

The proposed fracture network model will explicitly couple small-scale processes in individual fractures within fracture networks. This will complement existing large-scale dual-continuum models (e.g., the LLNL-developed reactive transport model, NUFT) by providing physically based, quantitative descriptions of subgrid-scale response to coupled geomechanics and reactive transport. This modeling approach will enhance our ability to predict complex field-scale behavior based upon laboratory measurements of coupled processes in individual fractures. The resulting model will provide a tool to rigorously explore the scaling behavior of these coupled processes in complex fractured systems.

\section{Mission Relevance}

Improved understanding of coupled geomechanical and geochemical alterations of fractured media is relevant to LLNL's role in supporting DOE energy and environmental missions, including geologic sequestration of carbon dioxide, enhanced oil recovery, engineered geothermal systems, and radioactive waste isolation.

\section{FY06 Accomplishments and Results}

Using our single-fracture model, in which the fundamental physics are represented explicitly, we completed development of a parallelized discrete fracture network model that allows detailed simulations in hundreds of interconnected fractures. We then enhanced the discrete fracture network flow model to include both reactive transport and mechanical deformation of individual fractures induced by stresses imposed on the fracture network. To explore the influence of network topology on coupled chemical-mechanical processes, we developed an algorithm for generating correlated random discrete fracture networks. Our simulations in two-dimensional networks of variable-aperture fractures quantify the effective permeability of the fractured medium under different stress conditions, fluid pressure gradients and fracture and network geometries. This approach is readily extendible to three-dimensional networks and provides a method for bounding the range 
of constitutive relationships for quantifying the connection between effective permeability of fractured rock masses and in situ stresses.

\title{
Publications
}

Detwiler, R. L., J. P. Morris, and S. M. Ezzedine, 2006. Coupling micromechanics and fluid flow through discrete fracture networks: quantifying effective permeability under variable stress conditions. EOS Transactions AGU, San Francisco, CA, Dec. 11-15, 2006.

UCRL-ABS-224243.

Detwiler, R. L., S. M. Ezzedine, and H. Rajaram, J. P. Morris, 2006. "The role of small-scale aperture variability on the formation of large-scale dissolution channels in rough-walled fractures." Proc. EOS Transactions AGU 85, 46. UCRL-ABS-215082.

Detwiler, R. L., and H. Rajaram, in press. "Predicting dissolution patterns in variable aperture fractures: evaluation of an enhanced depth-averaged computational model." Water Resour. Res. UCRL-JRNL-220815.

\section{Creating the Core Conditions of Extrasolar and Solar Giant Planets in the Laboratory}

Peter M. Celliers

\author{
04-ERD-065
}

\begin{abstract}
The goal of this proposal is to determine the properties of planetary materials in a pressure, density, and temperature regime that is critical to models of the giant planets. The key to a credible planetary model is an accurate equation of state (EOS) for its constituents at extreme conditions. We have developed a method that combines dynamic and static compression techniques using diamond anvil targets to reach relevant conditions. The technique will enable us to create material states that exist in planetary cores. We will load diamond anvil cells with hydrogen, helium, and hydrogen-helium mixtures at initial pressures of 1 to $5 \mathrm{GPa}$, and perform EOS (single and double shock) and transport measurements using a large laser as the shock driver. The same technique will be applied to other giant planet mixtures.

We expect to identify the insulating-conducting transition for the helium phase diagram at high density and electronvolt temperatures, and to generate similar data for the phase diagrams of hydrogen-helium mixtures. The data will provide important constraints on theoretical models of the EOS and conductivity of these fluids. The results may provide an unambiguous indication of helium phase separation in hydrogen-helium mixtures, and thus lead to a new understanding of the internal structure and evolution of Saturn and Jupiter. New high-pressure data on the melting curve of hydrogen will further constrain theoretical understanding of high-pressure hydrogen, particularly in relation to the metalinsulator transition.
\end{abstract}




\section{Mission Relevance}

Basic understanding of the properties of materials at high pressures and high temperatures, especially hydrogen isotopes, is key to the Lawrence Livermore's stockpile stewardship mission. This multi-institutional collaborative project also supports LLNL's basic science mission by furthering theoretical understanding of the interiors of giant planets.

\section{FY06 Accomplishments and Results}

In FY06, we performed 15 experiments on helium, hydrogen, deuterium, and hydrogenhelium mixtures. Six experiments on helium and five on hydrogen were performed to fill gaps in our data on the pure phases-this completed our survey of the Hugoniot states of both elements. Two experiments on hydrogen-helium mixtures provided enough data to prepare the first shock compression study of hydrogen-helium mixtures. Two experiments on deuterium provided additional validation of our techniques. During this project, we measured the density, temperature, pressure, and optical reflectivity along precompressed Hugoniots of helium and hydrogen to more than 2 Mbar, 14 times the liquid density, electron volt temperatures, and states that are electrically conducting. In addition, we produced the first-ever EOS and transport data for dense hydrogen-helium mixtures.

\section{Publications}

Celliers, P. M., et al., 2006. Equation of state and transport properties in dense helium. 6th Intl. Conf. High Energy Density Laboratory Astrophysics, Houston, TX, Mar. 11-14, 2006. UCRL-PRES-219641.

Lee, K. K. M., et al., 2006. "Laser-driven shock experiments on precompressed water: implications for 'icy' giant planets." J. Chem. Phys. 125, 014701. UCRL-JRNL-217068.

\section{The Large Synoptic Survey Telescope and Foundations for Data Exploitation of Petabyte Data Sets}

Kem H. Cook

$$
\text { 04-ERD-070 }
$$

\footnotetext{
Abstract

The next generation of imaging surveys in astronomy, such as the Large Synoptic Survey Telescope (LSST), will require multigigapixel cameras that can process enormous amounts of data read out every few seconds. This huge increase in data throughput over current capabilities calls for a new paradigm for extracting the knowledge content. We will develop the foundations for this new approach. In the process, we will produce significant scientific breakthroughs by developing new methods to probe both the elusive time and spatial variations in astrophysics data sets from the SuperMACHO (Massive Compact Halo Objects) survey, the Lowell Observatory Near-Earth Object Search (LONEOS), the Livermore Optical Transient Imaging System (LOTIS), and the Taiwanese American Occultation Survey (TAOS).
} 
This project continued to contribute to the development of the scientific foundations for future wide-field, time-domain surveys. Our algorithm and pipeline development provided the building blocks for the development of the LSST science software system. Our database design and performance measures have helped size and constrain LSST database design. LLNL has contributed significantly to the foundations of the LSST, which has significant applications for large-scale imaging and data-mining activities at LLNL. Significant scientific results have been released to the scientific community and more continue to be published and referenced, enhancing LLNL's scientific stature.

\section{Mission Relevance}

This project supports the national security mission by developing the LSST telescope and software and solving a variety of technical problems in imaging and data mining that are directly applicable to surveillance for nonproliferation. It also supports the DOE goal of understanding the nature and distribution of dark matter and the nature and evolution of dark energy.

\section{FY06 Accomplishments and Results}

In FY06 we (1) finished data collection for the SuperMACHO survey and published two related papers utilizing our prototype LSST database system and new anomaly detection algorithms; (2) published our discovery of new supernova light echo systems in the Large Magellanic Cloud in Nature; (3) continued investigating light echo systems in the Large Magellanic Cloud and elsewhere in the galaxy, resulting in the discovery of another unique evolved star similar to V838 Mon; (4) published a paper on the rise time of type Ia supernovae and developed a new algorithm for characterizing type Ia light curves, which constrains supernova explosion models; (5) continued to mine MACHO data discovering new R Coronae Borealis stars in the Milky Way bulge; (6) conducted TAOS data collection; (7) developed a pipeline for reduction of LONEOS new camera data and mined the LONEOS data for RR Lyrae variable stars extending our analysis of the Milky Way, discovering two distinct RR Lyrae populations that likely trace two distinct production mechanisms responsible for the Milky Way's halo; and (8) obtained all the MACHO data at LLNL for re-reduction.

\section{Publications}

Garg, A., et al., 2006. Pre-maximum lightcurves of type ia supernovae from the superMACHO project. 207th American Astronomical Society Mtg., Washington, DC, Jan. 8-12, 2006. UCRL-POST-218290.

Huber, M. E., et al., 2006. Mining the variables out of the superMACHO dataset. 207th American Astronomical Society Mtg., Washington, D.C., Jan. 8-12, 2006.

UCRL-POST-217927.

Kallivayalil, N., et al., 2006. "The proper motion of the large magellanic cloud using HST." Astrophys. J. 638, 772. UCRL-JRNL-214724. 
Rest, A., et al., 2005. "Testing LMC microlensing scenarios: the discrimination power of the superMACHO microlensing survey." Astronom. J. 634, 1103. UCRL-JRNL-204389.

Rest, A., et al., 2005. "Light echoes from ancient supernovae in the large magellanic cloud." Nature 438, 1132. UCRL-JRNL-216557.

Zaniewski, A., et al., 2005. "Discovery of five new R coronae borealis stars in the MACHO galactic bulge database." Astronom. J. 130, 2293. UCRL-JRNL-213036.

\title{
Mission to Very Early Earth
}

Ian D. Hutcheon

04-ERI-004

\begin{abstract}
This project will conduct a combined analytical and experimental program to study mineral and melt inclusions in ancient zircons (age $>4.2$ billion years) to determine when conditions suitable for life first emerged on Earth. After gathering the geochemical data as a function of zircon age, we will relate these data to the respective parent materials. The proposed work involves dating and isotopically and chemically analyzing the zircons and the mineral and melt inclusions in them, as well as a search for graphitic "chemofossils." The experimental portion focuses on the partitioning of trace elements, notably uranium and actinides, between zircon and melt. The investigation utilizes a broad array of new analytical facilities that support a variety of nuclear forensic applications.

The most important scientific output of this project is the chemical and isotopic characterization of Jack Hills zircons-the oldest extant piece of the Earth's early crust-as a function of their age. Based on these new data we will (1) understand how the fossil record of past life is preserved today, (2) determine the time when conditions suitable for supporting life first emerged on Earth, (3) understand the evolution of the atmosphere and hydrosphere over the first 400 million years of Earth's history-whether liquid water was present on the Earth's surface more than 4.4 billion years ago, and (4) develop a better understanding of crustal formation processes on Earth-like planets.
\end{abstract}

\section{Mission Relevance}

The project develops and enhances advanced microanalytical capabilities in support of LLNL's national security missions and advances its mission in basic science. In addition, the knowledge of the Earth's atmosphere and hydrosphere will benefit NASA and Department of Defense space missions. This project will also attract a cadre of young and highly trained scientists available for national-security-relevant work.

\section{FY06 Accomplishments and Results}

In FY06, we evaluated the partitioning behavior of rare earth elements between granitic melts and zircon using nanometer-scale secondary-ion mass spectroscopy imaging of 
synthetic zircon crystals grown in LLNL's petrology laboratory. However, the distribution of rare-earth elements was surprisingly complex, especially in view of the small size $(1-10 \mu \mathrm{m})$ of the synthetic zircons, which suggests that experimental conditions failed to achieve chemical equilibrium between melt and crystal. We also continued studies of Jack Hills zircons, which suggest oceans habitable to life and perhaps that continents could have appeared 400 million years earlier than previously believed. The titanium content of the zircons was found to range between 10 to 200 parts per million-values fully consistent with the suggestion of zircon crystallization at the relatively low temperatures $\left(<700^{\circ} \mathrm{C}\right)$ expected for hydrous, siliceous melts. Our work also revealed that trapped within these zircons are tiny mineral inclusions whose chemical compositions suggest active sedimentary processes and recycling of crustal material, requiring the presence of liquid water concomitant with the crystallization of the ancient zircon grains. Both the elevated titanium content and fractionated rare earth element contents of these zircons lend support to the hypothesis that the early Earth was cool. In short, this project found that the early Earth appears to have been more akin to a sauna than a cauldron.

\title{
Iodine-129 Accelerator Mass Spectrometry for Earth Science, Biomedical, and National Security Applications
}

\author{
Gregory J. Nimz 04-ERI-013
}

\begin{abstract}
This project will enable iodine-129 ( $\left.{ }^{129} \mathrm{I}\right)$ analysis by accelerator mass spectrometry (AMS) and build a foundation for its scientific application by developing chemical processing methods for environmental and nonproliferation use. During nuclear reprocessing, ${ }^{129} \mathrm{I}$ escapes to the environment, becoming an indicator of clandestine nuclear activity. Creating an AMS capability requires optimizing interrelated instrumental parameters and developing methods for low-level iodine chemical extraction. As proof of principle of our new capabilities, ${ }^{129}$ I in soil, groundwater, and plants will be analyzed. Initially we will focus on AMS instrument development, then on processing techniques, proof-of-principle demonstrations, and collaborative research.
\end{abstract}

This research is expected to result in (1) the ability to analyze small quantities of materials with low ${ }^{129} \mathrm{I} /$ iodine ratios; (2) a new generation of iodine-sampling techniques for low-level detection; (3) methods for iodine extraction from a wide range of natural materials (soil, groundwater, plants); and (4) an understanding of labile ${ }^{129}$ I migration in the environment. It will permit detection of clandestine nuclear activity and facilitate basic research in ecology, hydrology, agriculture, and human nutrition. Publishable results include newly developed AMS procedures, characterization of ${ }^{129} \mathrm{I}$ migration in soils (for determining moisture flux, colloid transport, and nutrient cycling), and ${ }^{129} \mathrm{I}$ hydrologic tracing methods (relevant to projecting effects of climate change on water resources).

\section{Mission Relevance}

This project provides techniques for nuclear forensics and nonproliferation in support of Lawrence Livermore's national security mission, develops technology for contaminant 
characterization in support of the Laboratory's mission in environmental management, and develops methods for ocean circulation analysis and biomedical research in support of the Laboratory's commitment to breakthroughs in fundamental science.

\title{
FY06 Accomplishments and Results
}

In FY06 we focused on a set of proof-of-principle demonstration projects that developed national security capabilities. We (1) completed a soil bomb-pulse ${ }^{129} \mathrm{I}$ effort, (2) furthered collaboration with researchers at the UC Merced using ${ }^{129} \mathrm{I}$ to characterize hydrology, (3) developed techniques for analysis of ${ }^{129}$ I in tree rings for assessing a region's nuclear reprocessing history, and (4) established analysis of small, low-ratio samples of oceanic pore-waters. Overall, this project developed advanced ${ }^{129}$ I methods for analysis of soils, surface waters, and trees, all of which are fundamental to the use of ${ }^{129} \mathrm{I}$ for nationalsecurity applications. We have prepared peer-reviewed journal articles as well as technique-development technical reports and conference papers that report on our results.

\section{Publications}

Brown, T. A., et al., 2004. Developments in the measurement of actinides and ${ }^{129} \mathrm{I}$ at LLNL by accelerator mass spectrometry. 45th Ann. Mtg. Institute of Nuclear Materials Management, Orlando, FL, July 18-24, 2004. UCRL-CONF-209000.

Zhao, P., et al., 2006. Distribution and transport of long-lived soluble fission products. MARC VII Mtg., Kailua-Kona, HI, Apr. 3-7, 2006. UCRL-ABS-216819.

Zhao, P., et al., in press. "Distribution of Tc-99 and I-129 in the vicinity of underground nuclear tests at the Nevada Test Site." J. Radioanal. Nucl. Chem. UCRL-CONF-220859.

\section{Carbon Flux in a California Grassland Soil Sequence: The Role of Dissolved Organic Carbon in Carbon Sequestration}

\author{
Christopher W. Swanston 04-ERI-014
}

\begin{abstract}
Soils have the capacity to stabilize large amounts of carbon. A thorough understanding of the factors that control this capacity is critical for defining input parameters used by models that predict climate change. The role of dissolved organic carbon (DOC) in carbon sequestration in soils is currently poorly understood. We propose to measure the carbon isotopic composition of DOC, bacterial biomass and soil organic matter (SOM) below annual grasslands on California coastal terraces to identify important transport pathways
\end{abstract}


and carbon cycling rates. The project will document compositional changes in DOC and SOM through the soil profile, which will complement the isotopic measurements. This research will result in accurate and comprehensive soil carbon cycling models at the site level.

We expect to quantify production and stabilization of DOC, identify important soil carbon cycling rates and pathways, measure mineralogical and seasonal effects on these cycling rates and pathways, and integrate flux measurements with a site-level soil carbon model. Insights into the role of DOC in transport and sequestration of soil carbon will improve the accuracy of input parameters needed by global carbon-cycling models.

\section{Mission Relevance}

The proposed research will enhance understanding of soil carbon cycling in support of LLNL's mission in environmental management, and it will provide valuable input for various DOE programs investigating the effects of increased levels of atmospheric carbon dioxide on the Earth's climate. The project will also strengthen LLNL collaborations with two UC campuses and Lawrence Berkeley National Laboratory.

\section{FY06 Accomplishments and Results}

In FY06, we finished sample collection, preparation, and analysis, finding carbon stabilization to be dominated by structural control in the upper soil and mineral control in the lower soil. This suggests to DOC fluxes, which show greatest attenuation with increasing depth and which move most young carbon from the upper soil to the deep soil during the winter months. Through our collaboration with the University of Colorado at Boulder we modified their site-level soil carbon model to incorporate measurements from our density fractionation of terrace soils. Through our collaboration with the U.S. Geological Survey (USGS), we obtained data for parameterizing our hydrological submodel and obtained access to a flume-outlet and catchment-sampling array, allowing an independent measurement of carbon cycling at one site for comparison against data from the site-level model. We also investigated-in collaboration with the USGS and UC Merced-the interaction of fire with the carbon cycle by conducting a controlled burn on one of the terraces. Data from samples collected before and after the burn will allow construction of a fire submodel in the carbon cycling model.

\section{Publications}

von Kiparski, G. R., et al., 2005. Soil carbon dynamics in a California coastal terrace chronosequence. 2nd Intl. Conf. Mechanisms of Organic Matter Stabilization, Moffett Field, CA, Oct. 9-13, 2005. UCRL-POST-219005. 


\title{
A New Capability for Regional, High-Frequency Seismic Wave Simulation in Realistic Three-Dimensional Earth Models to Improve Nuclear Explosion Monitoring
}

\author{
Arthur J. Rodgers 05-ERD-019
}

\begin{abstract}
We propose to perform a proof-of-concept study that will use model-based signals and coherent signal-processing to lower thresholds and improve confidence for seismic nuclear explosion monitoring. Currently, this monitoring is highly dependent on empirical observations of explosions and earthquakes and, therefore, spatially limited. In addition, inherent noise at seismic recording stations limits signal detection. Earth models derived from diverse data sets that include realistic stochastic heterogeneity will be used to compute theoretical seismograms. The model-based signals will be compared with a data stream to obtain optimal correlation and to detect weak signals.

This research addresses several challenges to current nuclear explosion monitoring technologies. Enabling the generation of Earth models for areas with few or no earthquakes or explosions will allow for monitoring of broad, uncalibrated areas. We expect that using coherent signal processing will drastically lower detection thresholds.
\end{abstract}

\section{Mission Relevance}

If successful, this project would provide new capabilities to support Lawrence Livermore's national security mission in ground-based nuclear explosion monitoring.

\section{FY06 Accomplishments and Results}

In FY06, we used a suite of model-based signals computed for three-dimensional Earth models to form the basis for correlation detection using the subspace method. To demonstrate the method, we modeled broadband regional seismograms for a moderate earthquake near the China-North Korea border. Synthetic seismograms were computed with the spectral element method for a suite of long-wavelength seismic velocity models inferred using a Markov Chain Monte-Carlo method. Results showed that coherent signalprocessing using the subspace method can be performed with synthetic seismograms computed from stochastic Earth models representing seismic velocity and density structure. Overall, this project established a proof-of-concept for a model-based, signalprocessing approach to seismic monitoring, with an emphasis on nuclear explosion monitoring. Further work will require higher-frequency synthetic seismograms and the inclusion of shorter-wavelength velocity structure, whether inferred from various seismic data sets or generated stochastically. 


\title{
Publications
}

Rodgers, A., D. Harris, and M. Pasyanos, 2006. A model-based signal-processing approach to nuclear explosion monitoring. 28th Seismic Research Review, Orlando, $\mathrm{FL}$, Sept. 19-21, 2006. UCRL-PROC-222691.

Rodgers, A., et al., 2005. "A model-based signal processing approach to seismic monitoring." EOS Trans. AGU 86(52), S32A-06. UCRL-ABS-214846.

\section{CHEMTREAT: Accelerated Remediation of Contaminated Fine- Grained Sediments by a Chemical Clay Cracking and Co-Solvent Flushing Process}

Ananda M. Wijesinghe 05-ERD-028

\begin{abstract}
Contaminants trapped in low-permeability, high-sorptivity, fine-grained clay sediments are inaccessible to advectively delivered treatment fluids, and are an unsolved, multibilliondollar problem in remediating groundwater contamination. We will investigate a process for chemically shrinking and cracking the sediments, and then flushing out the trapped contaminants using an effective chemical cracking agent (co-solvent) such as ethanol. We will perform laboratory experiments on synthetic and real clays to determine constitutive properties, crack propagation velocities, crack spacing and patterns, and methods of costeffectively delivering and recovering the chemicals in situ. Using these results, we will develop predictive models and codes for designing field remediation.
\end{abstract}

This project will (1) develop experimentally validated models for reliably predicting the performance of this novel combined chemical clay-cracking and contaminant remediation process and (2) define the design limits of the process. Building on results from a previous project that demonstrated feasibility of the basic concept, we will show that cracks can be created under confining stress by chemically induced shrinkage, and that the speed of cracking is not limited by the slow rate of diffusion of the cracking agent into the clay.

\section{Mission Relevance}

This project supports the Lab's environmental management mission by developing an effective method of remediating contaminated fine-grained sediments. This addresses a serious threat to national water resources and furthers the remediation of intractable contaminant sources at several DOE sites. Enhanced understanding of chemically induced cracking will also be applicable to designing waste containment facilities and predicting the performance of nuclear waste repository seals. 


\title{
FY06 Accomplishments and Results
}

In FY06, we (1) built a large (50-cm-diameter) flow cell and performed imaging experiments to determine crack density, spacing, and patterns as functions of confining stress, ethanol concentration, fluid chemistry, and clay type in a clay layer contacted and cracked by exposure to ethanol; (2) extended the constitutive property and crack velocity measurements initiated in $\mathrm{FYO5}$, developed solutions to overcome experimental errors, and characterized real clays from LLNL's Site 300 for use in FY07 experiments; and (3) made substantial progress in developing modeling concepts and computer codes to simulate observed spacing in the experimentally derived crack patterns.

\section{Proposed Work for FY07}

In FY07, we will (1) design, build, and perform a flow-and-transport laboratory experiment to evaluate the start-to-end performance of the clay-cracking remediation technology using the constitutive and cracking data on pure clays and clay-sand mixtures measured in FY06, as well as using our theoretical models and computer codes; (2) evaluate the ability to deliver the cracking agent (ethanol) without dilution to the targeted clay zone, to rapidly crack the target zone to the full depth of contamination, to mobilize the contaminants into the advecting solvent flow, and to capture and extract the contaminants without excessive loss; and (3) perform additional constitutive and crack-velocity tests on sand-clay mixtures to better define design limits for the CHEMTREAT technique.

\section{Controls of Fluid Chemistry on Fracture Growth}

\section{Carol J. Bruton 05-ERD-035}

\begin{abstract}
The role of water and its dissolved content in fracturing and rock deformation is poorly understood. However, new models describing the controls of surface chemistry on fracturing, and the relation between fracturing and breakage of bonds during mineral dissolution, suggest new ways to quantify the impact of fluid chemistry. We are using the hydrothermal atomic force microscope (HAFM) to image fracture growth as a function of fluid chemistry and $\mathrm{pH}$ at subsurface temperatures. The project goal is to modify the HAFM to quantify these effects and advance our understanding of the role of solute-bearing water on rock deformation. This knowledge can then be used to predict and engineer fracture growth in subsurface materials as a function of their environment.

The expected outcome of this project is a new, unique capability that can be used to study subcritical crack growth (SCG) in minerals, rocks, and other materials. We expect to break through the $80^{\circ} \mathrm{C}$ experimental barrier that has limited previous experimental efforts in this area and link surface chemistry and rock mechanics to make significant interdisciplinary advances. Results of this research will be published in peer-reviewed journals.
\end{abstract}




\title{
Mission Relevance
}

This work supports Lawrence Livermore missions in energy security and carbon management through its potential applications to DOE programs in fossil and geothermal energy. It also has significant implications for repository science and material lifetimes. This work could lead to breakthroughs in fundamental science and technology that will attract new talent to the Laboratory.

\section{FY06 Accomplishments and Results}

In FY06, we modified the HAFM to optimize its use for SCG studies. We (1) designed a Kalrez ${ }^{\circledR}$ membrane that permits a wider $z$-range of motion in the HAFM, (2) designed a longer-range piezo tube that extends the $x-y$ scanning range by a factor of nearly four, and (3) replaced the contact-only (two-detector) optical head with a top-view, atomic force/lateral force microscopy (four-detector) optical head that permits frictional force measurements. We confirmed the performance of the bending jig using three-dimensional finite element modeling to simulate bending and calculate the stress. We completed project objectives by producing in situ, real time images of SCG in silica glass in the HAFM as a function of changing solution $\mathrm{pH}$, and we generated a movie of the SCG process.

\section{Integration and Codevelopment of a Geophysical Carbon Dioxide Monitoring Suite}

\section{Samuel J. Friedmann 05-ERD-038}

\begin{abstract}
As geological carbon sequestration increases in importance as a carbon management strategy, a need exists for subsurface monitoring, measurement, and verification (MMV) tools. In particular, inexpensive, non-intrusive, and well-calibrated approaches are required. We propose using the Lab's Stochastic Engine (a Monte Carlo, Markov-Chain algorithm) to compare and simultaneously invert data from three orthogonal geophysical approaches. This will provide alternative approaches to subsurface carbon-dioxide $\left(\mathrm{CO}_{2}\right)$ monitoring as well as oil field flow-front management.

If successful, we expect to be able to determine the distribution and concentration of subsurface $\mathrm{CO}_{2}$ using these combined, jointly inverted data sources. This approach would greatly improve risk characterization associated with $\mathrm{CO}_{2}$ storage and provide potential operators, regulators, and petroleum producers with information to manage $\mathrm{CO}_{2}$ injection and storage. Additional significance would come from an improved scientific understanding of subsurface processes and uncertainties (e.g., earth heterogeneity).
\end{abstract}




\section{Mission Relevance}

The research supports a variety of DOE geologic carbon-sequestration efforts and is strongly aligned with areas of the Lab's energy technology and environmental management missions, including carbon management and environmental risk reduction.

\section{FY06 Accomplishments and Results}

In FY06 we (1) collected and interpreted data from the Salt Creek field $\mathrm{CO}_{2}$ flood in Wyoming; (2) completed the industrial site shared-Earth model at Teapot Dome, Wyoming; (3) reworked data from Salt Creek, reducing the injection signal data enormously; (4) presented our results at major national and international conferences; (5) conducted numerical experiments that demonstrated the model's ability to use disparate data types to improve predictions of subsurface plume prediction and monitoring; and (6) identified an industrial site for future application of the technology and entered a cooperative research and development agreement with an industrial partner. DOE's National Energy Technology Laboratory and FutureGen Program and the Weyburn $\mathrm{CO}_{2}$ Monitoring and Storage Project have expressed interest in pursuing this technology.

\section{Publications}

Ramirez, A., et al., 2005. Joint reconstructions of subsurface plumes multiple data types. SPE Advanced Tech. Workshop $\mathrm{CO}_{2}$ Sequestration, Galveston, TX, Nov. 15-17, 2005. UCRL-ABS-216477.

Ramirez, A., et al., 2006. Site characterization using joint reconstructions of disparate data types. $\mathrm{CO}_{2}$ Site Characterization Symp., Berkeley, CA, Mar. 20-22, 2006.

UCRL-PROC-218592.

Ramirez, A., et al., 2006. Subsurface imaging of $\mathrm{CO}_{2}$ plumes using multiple data types and Bayesian inference. 5th Annual Conf. Carbon Capture and Sequestration, Alexandria, VA, May 8-11, 2006. UCRL-ABS-218591.

Ramirez, A., et al., 2006. Joint reconstructions of subsurface $\mathrm{CO}_{2}$ plumes using a Markov chain Monte Carlo approach. GHGT-8 8th International Conf. Green House Gas Control Technologies, Trondheim, Norway, June 19-22, 2006. UCRL-ABS-215765.

\section{Detection and Attribution of Regional Climate Change}

\section{Govindasamy Bala 05-ERD-042}

\section{Abstract}

Detection, and attribution to human causes, of climate change requires that we demonstrate that the observed changes cannot be accounted for by the natural variability of the climate system. Past studies have investigated the detection and attribution problem 
on a global or continental scale. Our project is investigating this problem for the first time on a subcontinental scale, in the western U.S. Our goal is to examine the dependence on model resolution of natural variability and trends in proposed climate-change detection variables. We believe that climate change research in the future will increasingly focus on regional climate change, and our work will help LLNL to establish leadership in the area of regional climate assessment.

Because the consequences of global energy production and use will appear first at the regional level, it is important to investigate regional climate detection and attribution. Our investigations will help determine whether past climate change in the western U.S. can be attributed to historical forcings, and whether regional and local forcings are important in regional climate changes. Results from a suite of simulations performed in our work will increase our confidence in predicting future changes and help address other regional-scale phenomena of great societal impact (e.g., water resources, health, recreation, agriculture, ecosystems). Papers describing our high-resolution model results will be submitted for publication in peer-reviewed journals.

\section{Mission Relevance}

The simulations performed in this study formed the foundation for regional-scale analyses of carbon and air chemistry that support LLNL's energy and water security missions and the DOE's missions in environmental management and climate change.

\section{FY06 Accomplishments and Results}

In FY06, we completed the first-ever high-resolution global climate simulation using the National Center for Atmospheric Research's Community Climate System Model 3. Two stable simulations were performed-a 1,000-year pre-industrial simulation and a 400-year present-day simulation. Scientific papers based on the improvements in our solutions were published. Our simulations also provided 20-year-boundaried conditions for a regional mesoscale model with a spatial resolution of $\sim 10 \mathrm{~km}$. Our achievements will help determine whether historical climate change in the western U.S. can be attributed to our best estimate of historical forcing. This work will help understand whether regionalscale forces are important to regional detection of climate change.

\section{Publications}

Bala., G., et al., 2006. CCSM3 FV $1 \times 1.25$ simulations for the detection and attribution of regional climate change in the Western USA. UCRL-PRES-222009.

Barnett, T., et al., 2006. LUSCiD-LLNL-UCSD/SIO scientific data project: climate studies. UCRL-PRES-223662.

McClean, J., et al., 2006. "The fidelity of the ocean and ice in a new CCSM simulation with a finite volume dynamical core in a $1 \times 1.25$ atmosphere." Eos Trans. AGU 87(52).

UCRL-ABS-224250. 
Mirin, A., and G. Bala, 2006. Coupled finite-volume simulations at one-degree resolution. UCRL-PRES-219702.

Mirin, A., et al., 2006. "CCSM simulations using a finite-volume numerical method for the atmospheric model: present-day climate." Eos Trans. AGU 87(52). UCRL-ABS-224252.

\title{
A Dynamically Coupled Groundwater, Land Surface, and Regional Climate Model to Predict Seasonal Watershed Flow and Groundwater Response
}

Reed M. Maxwell

\author{
05-ERD-043
}

\begin{abstract}
This project will develop a new model that couples three distinct submodels for climate, land surface hydrology, and groundwater flow that are traditionally used independently with oversimplified boundary conditions. It is hoped that the new model will yield a better understanding of important mass and energy couplings and afford more accurate predictions of seasonal watershed flow and groundwater response to precipitation. We will demonstrate cases where the simplified lower-boundary condition present in the regional climate model is not a reasonable approximation and that interactions among the atmosphere, land surface, and the subsurface have an impact upon mass, energy, and momentum balances. We also will investigate to what extent practical and stability considerations affect the dynamic coupling of these models, and how the models adequately represent the physical processes and observations within the watershed.
\end{abstract}

We expect that the coupled model will lead to (1) an improved capability to simulate the physical processes through which the subsurface and precipitation are coupled, (2) a new tool for predicting surface water and groundwater hydrology at the watershed scale on a seasonal timeframe, (3) a simulation of hydrologic response of a watershed that will provide insight into hydrologic forecasting, and (4) a more realistic representation of weather predictions at the regional scale.

\section{Mission Relevance}

This project will contribute to the evolution of regional climate, land surface, and groundwater models in support of the DOE's environmental management and climate change missions, and lead to breakthroughs in energy and water security research.

\section{FY06 Accomplishments and Results}

In FY06, we (1) fully coupled (in parallel) the groundwater/overland flow model ParFlow, the regional climate model ARPS, and the land surface model ISBA; 2 ) conducted a number of tests to ensure these models are balancing water and energy and behaving in a stable and predictable manner; (3) investigated parallel scaling of the model and 
the stability and computational efficiency of the operator-splitting approach used; and (4) performed simulations over a wide range of spatial scales with varying resolution to investigate differences between the fully coupled and uncoupled behavior of the planetary boundary layer. This project produced a new computational tool that is capable of investigating links between precipitation, overland flow, and groundwater at a level of detail that was not previously possible, and has resulted in high-profile, peer-reviewed journal articles and papers at major conferences.

\title{
Publications
}

Chow, F. K, et al. 2006. Effects of soil moisture heterogeneity on boundary layer flow with coupled groundwater, land-surface, and mesoscale atmospheric modeling. American Meteorological Society 17 th Symp. Boundary Layers and Turbulence, San Diego, CA, May 22-26, 2006. UCRL-CONF-224877.

Kollet, S. J., and R. M. Maxwell, 2006. "Integrated surface-groundwater flow modeling: a free-surface overland flow boundary condition in a parallel groundwater flow model." Adv. Water Resour. 29, 945. UCRL-JRNL-211468.

Maxwell, R. M., et al., 2006. Simulating water and energy fluxes using a coupled groundwater, surface water, land surface and regional climate model. 86th Ann. Mtg. American Meteorological Society, Atlanta, GA, Jan. 29-Feb. 2, 2006. UCRL-POST-218459.

\section{Developing a Reactive Chemistry Capability for the NARAC Operational Model (LODI)}

Philip J. Cameron-Smith 05-ERD-050

\begin{abstract}
Atmospheric chemistry can significantly alter the impact of many chemical releases, such as chlorine and nerve agents. To address the current imbalance between the dispersion and chemistry capabilities of LODI-the operational response model used by LLNL's National Atmospheric Release Advisory Center (NARAC)-we are adding a reactive chemistry and aerosol capability to solve an arbitrary network of chemical reactions and the in situ evaporation and condensation of aerosols. Improvements will include the ability to read time- and space-dependent ambient concentrations of relevant species from IMPACT, LLNL's global atmospheric chemistry and aerosol model.

The addition of a reactive chemistry capability to LODI, plus interfacing with the IMPACT global atmospheric chemistry code, will create a unique, emergency-response capability that will greatly enhance our ability to plan for, and respond to, terrorist attacks and industrial accidents that involve reactive chemistry, including many chemical agents and toxic industrial chemicals. This work will also facilitate development of detection and monitoring plans for factories and laboratories, including clandestine facilities. The resulting model also will have a dual use in local and regional air-quality studies.
\end{abstract}




\section{Mission Relevance}

By improving NARAC's operational emergency response capabilities, this project supports a range of national security and homeland security missions. Chemical dispersion and fate are key components for managing response to terrorist attacks. Chemical fate also has an important role in understanding proliferation signatures as well as pre- and post-strike consequence evaluation of military targets.

\section{FY06 Accomplishments and Results}

In FY06, we integrated our Gear code (a solver for an arbitrary network of chemical reactions) into the LODI emergency response code, along with a new semi-Lagrangian advection scheme that is compatible with the existing Lagrangian framework. We also completed a test problem simulating the release of a chemical weapon surrogate with a real windfield, an $80 \times 80 \times 80$ grid, and 4 million semi-Lagrangian air parcels tracking 13 chemical species. The expected chemical degradation was seen in the simulations, with the initial chemical progressively broken down into a cascade of intermediate products before yielding the final products. Using 32 processors on Livermore's Multiprogrammatic Capability Resource supercomputer, simulation time for this problem of computationally realistic size was about 10 minutes, which indicates that our code is fast enough for use in codes that provide operational response.

\section{Proposed Work for FY07}

In FY07, our focus will be on validating the capabilities we added to LODI using observations of both real releases and air-quality simulations. Because data on real releases are limited in quantity and quality, we will use air-quality data (both observations and emission databases, which are available in abundance) to conduct simulations to validate the general model capabilities; this will be a particularly challenging test of our chemistry transport models. We will also run hypothetical scenarios to showcase our new capabilities for simulating terrorist attacks, industrial accidents, and battlefield events.

\section{Enhanced Isolation Performance of Geologic Carbon Dioxide Storage Sites through Mineral Trapping: Experimental and Field Confirmation of Model Predictions}

James W. Johnson

$$
\text { 05-ERD-054 }
$$

\footnotetext{
Abstract

Our recent reactive transport modeling of engineered geologic carbon-dioxide $\left(\mathrm{CO}_{2}\right)$ storage predicts that influx-triggered carbonate precipitation may ultimately lead to significantly reduced permeability within typical shale cap rocks, thereby significantly improving their long-term hydrodynamic seal capacity-the single most important
} 
constraint on long-term isolation performance. This project proposes to perform a multiscale confirmation of this key model prediction by conducting integrated reactivetransport modeling and batch-reactor experiments on the laboratory benchtop scale and by modeling the formation of the best-characterized natural $\mathrm{CO}_{2}$ reservoir (McElmo Dome) on the field scale.

Our recent modeling work on geologic $\mathrm{CO}_{2}$ storage in saline aquifers demonstrates unparalleled expertise in this emerging simulation arena. Experimental and field confirmation of our most significant model predictions regarding mineral trapping-which have crucial implications for the isolation performance of geologic $\mathrm{CO}_{2}$ storage siteswill provide a critical measure of confidence in our unique reactive transport modeling capabilities. Hence, success in this project will further enhance LLNL's capabilities in reactive transport modeling applied to geologic $\mathrm{CO}_{2}$ storage.

\title{
Mission Relevance
}

Engineered geologic $\mathrm{CO}_{2}$ sequestration represents the most promising near-term emissions-reduction strategy for carbon management. The multiscale confirmation of key model predictions for $\mathrm{CO}_{2}$ sequestration performed in this project supports the LLNL mission in energy security and is well aligned with DOE programs in $\mathrm{CO}_{2}$ sequestration.

\section{FY06 Accomplishments and Results}

In FY06, we (1) completed baseline experimental modeling, (2) conducted the designed experiment as well as post mortem analysis and model calibration, (3) obtained McElmo mineralogy from new core analyses by x-ray diffraction, and (4) extracted a representative section of our McElmo EarthVision (EV) model for import into the LLNL-developed reactive transport model NUFT. This project was significant in that we (1) created a new experimental capability and NUFT model for assessing $\mathrm{CO}_{2}$-fluid-rock interactions and dependent permeability evolution-follow-on work has been funded under DOE's Weyburn Enhanced Oil Recovery Project; (2) acquired a geologic model and core for McElmo, imported them to EV, and extracted a section for import to NUFT; (3) obtained mineralogy from new core analyses; and (4) obtained overall results confirming our original hypothesis that geochemical reactions tend to reduce shale permeability and lead to perditions of enhanced caprock efficacy.

\section{The Physics of Recombining Plasmas in Celestial Sources}

\author{
Gregory V. Brown 06-ERD-010
}

\section{Abstract}

This project studies the recombination processes-charge exchange and radiative recombination-that follow collisions between highly charged ions and neutral atoms or 
free electrons. We will determine the spectral signature of photon emission following recombination reactions between astrophysically relevant neutral gases and bare and hydrogenic ions, with an emphasis on iron. These results will be used to identify the contributions from recombination processes in both celestial and fusion plasma sources. This project combines several unique LLNL capabilities-including the Super Electron Beam Ion Trap (SuperEBIT), a source of highly charged ions, and gas- and laser-injection systems, each for introducing neutral material into the trap-along with a high-resolution microcalorimeter from the National Aeronautics and Space Administration for the detection of $x$ rays.

We will provide the first-ever high-resolution spectral signature of $\mathrm{x}$-ray emission following charge-exchange recombination as a function of the donor gas material and of the target ion's atomic number. In addition, we are implementing a new method for analyzing spectra-the Photon Clean Method (PCM)-which uses a novel Monte Carlo approach to spectral fitting that raises the probability of discovering weak $\mathrm{x}$-ray production mechanisms that may contribute line emission to a spectrum. Our results will help to diagnose physical processes taking place in a variety of celestial sources, including the Galactic Ridge and Center, low-mass x-ray binaries, and supernova remnants, in addition to complex laboratory sources such as tokamaks.

\section{Mission Relevance}

Because the thermonuclear phenomena found in stars are the same as those that occur in manmade nuclear explosions, this project will benefit numerical simulation and fundamental experimental research used in stockpile stewardship, in support of our national security mission.

\section{FY06 Accomplishments and Results}

In FY06, we (1) produced the first-ever high-resolution measurements of $\mathrm{x}$-ray spectra produced by neutral nitrogen, helium, and hydrogen recombining with bare and hydrogenic iron ions via charge exchange; (2) compared our results to the x-ray spectra of the Galactic Center measured by the Suzaku X-Ray Observatory and found a discrepancy that appears to rule out charge exchange as the source of its $x$-ray emission; ( 3 ) installed an atomic hydrogen source onto SuperEBIT; and (4) used PCM and our charge-exchange spectra to analyze the $x$-ray spectrum of Comet 73P Schwassmann-Wachmann 3 measured with the Swift Gamma-Ray Burst Explorer's x-ray telescope.

\section{Proposed Work for FY07}

We will (1) complete a temperature-diagnostic spectrometer, (2) use this spectrometer to revisit previous measurements with different neutral gas injection pressures to quantify the temperature dependence of our spectra and make a more accurate comparison with theory, (3) measure the x-ray spectra produced by charge exchange between atomic hydrogen and astrophysically relevant ions, and (4) use PCM to analyze Suzaku data on the Galactic Ridge and Center and test if PCM could have applicability to homeland security problems, including detection of man-made radioisotopes. 


\title{
Publications
}

Figueroa-Feliciano, E., et al., 2006. "Science with Micro-X: the TES microcalorimeter x-ray imaging rocket." Proc. SPIE 6266, 62660A. UCRL-PROC-221634.

Kelley, R. L., et al., in press. "The Suzaku high-resolution x-ray spectrometer." Publ. Astron. Soc. Jpn. UCRL-JRNL-224669.

Koyama, K. et al., in press. "Iron and nickel line diagnostics for the galactic center diffuse emission." Publ. Astron. Soc. Jpn. UCRL-JRNL-224878.

Wargelin, B., et al., 2006. "Charge exchange spectra of hydrogenic and He-like iron." Astrophys. J. 634, 687. UCRL-JRN-211937.

\section{Urban Atmospheric Turbulence: Improved Turbulence Closure Models through Observations and Simulations}

Julie K. Lundquist 06-ERD-026

\begin{abstract}
We propose to test advanced turbulence models and numerical methods for urban simulations then integrate them into a numerical model that is widely used for weather prediction and transport as well as dispersion prediction. This project is motivated by the inadequacy of current turbulence models (compared to observations) and the demand for complex time- and space-varying boundary conditions for urban-scale models. Improved models are essential for national security purposes in the case of accidental or intentional urban atmospheric release of hazardous materials. Project success will be determined by comparing dispersion simulations to a refined field dataset from the Joint Urban (JU) 2003 Atmospheric Dispersion Study.
\end{abstract}

The new, accurate turbulence models for urban areas developed in this project will provide improved plume predictions for national security applications. The urban numerical methods will allow the seamless integration of urban-scale simulations with appropriate coarser-scale simulations to provide the best available forcing of urban-scale models. This project will ensure that simulations for emergency response, sensor siting, and forensic studies in urban areas are of the greatest fidelity possible for the protection of urban populations.

\section{Mission Relevance}

Our project supports LLNL's national and homeland security missions by improving capabilities to provide emergency response for atmospheric releases of hazardous materials. 


\section{FY06 Accomplishments and Results}

In FY06, we (1) completed an analysis of atmospheric turbulence and its relationship with larger-scale motions during the $\mathrm{JU}$ experiment and presented results of our analysis at a professional meeting and in a journal publication; (2) brought the Weather Research and Forecasting Model (WRF) to LLNL, ran it on an LLNL supercomputer, and archived the boundary condition datasets; (3) modified the WRF to output turbulence kinetic energy (TKE) and compared WRF-TKE predictions to observations (results indicated that improved turbulence models are required); (4) integrated the Dynamic Reconstruction Model (DRM) with WRF's structure; and (5) began improving WRF's surface-characterization scheme by implementing the immersed boundary method in two dimensions.

\section{Proposed Work for FY07}

In FY07, we will (1) document the improvements DRM made in dispersion, wind field, and turbulence parameters on $100-\mathrm{m}$ scales by simulating flow and dispersion during two JU intensive-operation periods, comparing results from DRM turbulence and existing turbulence models; (2) share the integrated WRF-DRM with the atmospheric science community via a new WRF distribution, and provide guidance for the appropriate scale transition between DRM and traditional turbulence models; and (3) complete implementation of the immersed boundary method and generate both regional grids and urban grids for WRF simulations using the immersed boundary method.

\section{Publications}

Lundquist, J. K., and J. D. Mirocha, 2006. Interaction of nocturnal low-level jets with urban geometries as seen in Joint Urban 2003 data. American Meteorological Society 6th Symp. Urban Environment, Atlanta, GA, Jan. 29-Feb. 2 2006. UCRL-CONF-216884.

Lundquist, J. K., and J. D. Mirocha, 2006. Interaction of nocturnal low-level jets with urban geometries as seen in Joint Urban 2003 data. American Meteorological Society 6th Symp. Urban Environment, Atlanta, GA, Jan. 29-Feb. 2 2006. UCRL-CONF-213696.

Lundquist, J. K., and S. T. Chan, in press. "Consequences of urban stability conditions for computational fluid dynamics simulations of urban dispersion." J. Appl. Meteorol. Climatol. UCRL-JRNL-217489.

Mirocha, J. D., et al., 2006. Implementation of the dynamic reconstruction subfilter turbulence model and immersed boundary method for improved large-eddy simulation of flow over complex terrain. 2006 Weather Research and Forecasting Users Workshop, Boulder, CO, June 19-22, 2006. UCRL-POST-222068.

Mirocha, J. D., et al., 2006. Regional- to urban-scale influences on atmospheric turbulence and dispersion. UCRL-POST-218872. 


\title{
Atmospheric Carbon-14 Dioxide Constraints on and Modeling of Net Carbon Fluxes
}

\author{
Thomas P. Guilderson 06-ERD-031
}

\begin{abstract}
Carbon dioxide $\left(\mathrm{CO}_{2}\right)$ is one of the most important anthropogenic greenhouse gases influencing global climate. Sources and sinks of $\mathrm{CO}_{2}$ impart their signature on the distribution, concentration, and isotopic composition of $\mathrm{CO}_{2}$, while spatial and temporal variability provide information on net surface fluxes. Observations of carbon and oxygen isotope tracers and their rate of change in the atmosphere can be used to constrain global and regional contributions of different carbon sources and sinks, because each of these sources and sinks bears a different isotope or elemental ratio signature. We propose to measure carbon-14 dioxide samples from a suite of North American locations and use these results in inversion estimates to constrain unidirectional carbon fluxes.

This project is expected to produce the best possible estimates of surface carbon sources and sinks using atmospheric observations of $\mathrm{CO}_{2}$ partial pressure, carbon-13 and carbon14 abundances, and oxygen/nitrogen ratios; atmospheric transport based on a suite of Atmospheric Tracer Transport Model Intercomparison (TransCom) results; and the best possible representation of isotopic composition of exchangeable carbon pools and associated isotopic fractionation factors.
\end{abstract}

\section{Mission Relevance}

By investigating size and variability of the dynamic reservoirs and fluxes of carbon within the Earth system, this research supports Laboratory missions in energy security and longterm energy needs. The synergy between field data and computational models offered by this project will contribute to DOE's missions in energy security and carbon management.

\section{FY06 Accomplishments and Results}

In FY06, we (1) acquired, in collaboration with Purdue University, the TransCom model for inverting annual model results, and ran the model to reproduce TransCom3 Level-1 output; (2) acquired all emissions input required for a TransCom-3 Level1 simulation, and wrote a regridding routine in Iterative Data Language to spatially aggregate high-resolution emissions onto a coarser grid for LLNL's Integrated Massively Parallel Atmospheric Chemical Transport (IMPACT) model; (3) modified IMPACT to allow for temporal interpolation of monthly emissions as required by TransCom-3 Level-1 specifications; (4) measured carbon-14 dioxide in vertical profiles over Niwot Ridge, Colorado; and (5) continued to analyze the Scripps Institution of Oceanography clean-air archive. In addition, our first stratospheric samples, which were collected in 2004, were analyzed to document the "natural" production end-member. 


\section{Proposed Work for FY07}

In FY07, we will (1) continue making carbon-14 measurements for archived, stratospheric, and exploratory vertical profile samples obtained from our collaborators; (2) use the TransCom inverse modeling system to infer surface radiocarbon fluxes with the transport fields from forward models and with a synthetic carbon-14 dataset; (3) show how carbon14 fluxes can be used to separate inferred net carbon-12 fluxes into their photosynthetic and respiratory components; and (4) incorporate data into the inversion solutions as they are generated.

\section{Publications}

Graven, H. D., T. P. Guilderson, and R. F. Keeling, 2006. High-precision AMS-14C measurements on atmospheric $\mathrm{CO}_{2}$ samples at CAMS. 19th Intl. ${ }^{14} \mathrm{C}$ Conf., Oxford, UK, Apr. 3-7, 2006. UCRL-ABS-218357.

Graven, H. D., T. P. Guilderson, and R. F. Keeling, in press. "Methods for high precision ${ }^{14} \mathrm{C}$ AMS analysis of atmospheric $\mathrm{CO}_{2}$ at LLNL." Radiocarbon. UCRL-JRNL-225389.

\section{Regional Climate}

David C. Bader

$$
\text { 06-ERD-066 }
$$

\footnotetext{
Abstract

Understanding regional climate depends on gaining knowledge about natural climate variability at similar scales. The proposed research will contribute to this understanding by (1) developing an integrated, multimodel capability for regional climate change impact and adaptation studies and (2) affording a model-based statistical analysis of natural climate variability over regions as small as watersheds. This project will examine the highresolution natural variability for California in a 20 -year segment from a 700 -year control climate simulation experiment using downscaling methods as well as information from the original coupled model simulation to evaluate the strengths and weaknesses of the approaches.
}

This project will use observations of atmospheric variables to examine the ability of climate models to simulate observed variability. The project will leverage LLNL's climate-modeling expertise to further the analysis of California climate change.

\section{Mission Relevance}

In support of LLNL's missions in energy and water security and environmental management, we will develop an integrated, multimodel capability for regional climate change analysis and for impact and adaptation studies. This suite of simulation tools will provide a new capability for managing regional water resources in California that can be applied to other regions in the U.S. 


\title{
FY06 Accomplishments and Results
}

After a midyear start, we designed a prototype experiment to simulate regional climate over a 20-year period in California. Two different approaches were explored for scaling down, to the higher resolutions needed for regional analysis, the output from an existing moderate-resolution 700-year global coupled atmosphere-land surface-ocean sea ice climate model simulation. The first was a 20 -year simulation performed with a $0.5^{\circ} \times$ $0.625^{\circ} \mathrm{global}$ atmospheric general circulation model driven by sea-surface temperature data from the coupled simulations. The second approach was a 20-year simulation with a limited-area mesoscale model running at $9 \times 9 \mathrm{~km}$ resolution over the western United States. Unanticipated problems with the file system on the Thunder supercomputer prevented the completion of the simulations by the original target date of July, but the two downscaling runs were finished in September.

\section{Proposed Work for FY07}

The first task for FY07 will be to perform the analysis task originally scheduled for FY06. Complete climatologies from the three models will be compared to long observational records of surface and (when possible) atmospheric climate variables-specifically, those relevant to surface temperature and precipitation. Next, we will complete the major task for FY07-to take the lessons learned from this prototype simulation and develop a sustained capability in high-resolution dynamical downscaling that complements LLNL expertise in global simulation. For this task, we will switch to the community Weather and Research Forecasting modeling system because of its status as a broadly supported community model.

\section{Development of Integrated Microanalysis of Nanomaterials}

John P. Bradley

06-ERI-001

\begin{abstract}
In recent years, there have been significant advances in detection and imaging capabilities using electron microscopy and ion microprobe techniques. We propose to develop an integrated microanalysis capability utilizing these advances with state-of-the-art instrumentation at Lawrence Livermore to enable a new level of investigations into the mineralogical, chemical, and isotopic properties of nanomaterials. The initial development will be carried out on natural nanomaterials captured at hypervelocity speeds by lowdensity silica aerogels. The analytical studies of these captured particles will provide new insights into cosmically primitive extraterrestrial material and develop synergistic capabilities that are complimentary to multiple mission-relevant analytical needs.

The National Aeronautics and Space Administration Stardust samples are the first solidmatter materials to be returned to Earth since the Apollo missions of the 1970s. New insights into early solar system processes will be gained by analyzing these "fresh" materials in contrast to analyzing materials (e.g., interplanetary dust particles) that have encountered space weathering and atmospheric entry alteration before being analyzed
\end{abstract}


in a laboratory setting. Stardust samples will enable comparisons between astronomical spectral observations of comets and laboratory observations of astromaterials. Stardust samples will give insights into whether comets played an important role in bringing life to early Earth, and whether cometary dust is composed of interstellar dust, (inner) solar system dust, or both.

\section{Mission Relevance}

The proposed work will develop and investigate new analytical techniques for nanoscale materials characterization that are directly applicable to Lawrence Livermore's missions in stockpile stewardship and homeland security. The project also supports the Laboratory's mission in breakthroughs in fundamental science and applied technology, specifically in the area of astrophysics and space science, which includes exploration of Kuiper Belt objects such as Comet Wild 2.

\section{FY06 Accomplishments and Results}

Following the successful return of the Stardust sample-return capsule in January 2006, we began extracting and analyzing cometary samples embedded in silica aerogel and in micro-craters in aluminum foil. For the analysis, we used transmission electron microscopy (TEM), focused ion beam-field emission scanning electron microscopy, and synchrotron beamlines at the Stanford Synchrotron Radiation Laboratory and the Advanced Light Source. We also compared the analytical capabilities of LLNL's new Super Scanning TEM (SuperSTEM) system with synchrotron-based techniques. We found that although comets were formed far beyond the orbit of Neptune, these cometary samples appear to be full of complex minerals and material from the inner solar system and from close to the Sun, with large variations from particle to particle. Preliminary results from the analysis of Stardust material were presented at both the Lunar and Planetary Science Conference in Houston and at a workshop held in California sponsored by the University of California Institute of Geophysics and Planetary Physics.

\section{Proposed Work for FY07}

In FY07, we plan an in-depth exploration of the properties of Stardust cometary particles to gain new insights into early solar processes. Specifically, we will study the mineralogical and chemical composition of the particles using SuperSTEM. Chemical studies will focus on studying the bulk and trace compositions of the cometary particles using both synchrotron $x$-ray fluorescence and TEM-energy-dispersive $x$-ray analysis. The studies of cometary material will be supported by laboratory simulations that will include ion implantation of surrogate material and molecular dynamic modeling to investigate nanoscale hypervelocity impact processes.

\section{Publications}

Brennan, S., et al., 2006. Synchrotron total-reflection $x$-ray fluorescence (SR-TXRF) of Genesis return samples. 37th Lunar and Planetary Sciences Conf., League City, TX, Mar. 13-17, 2006. UCRL-ABS-219146. 
Burchell, M. J., et al., 2006. "Identification of minerals and meteoritic materials via Raman techniques after capture in hypervelocity impacts on aerogel." Meteoritics Planet. Sci. 41(2), 217. UCRL-JRNL-207273.

Floss, C., et al., 2006. "Identification of isotopically primitive interplanetary dust particles: a NanoSIMS isotopic imaging study." Geochim. Cosmochim. Acta 70, 2371. UCRL-JRNL-215066.

Flynn, G. J., et al., 2006. Chemical analysis of Wild-2 samples returned by stardust. 37th Lunar and Planetary Sciences Conf., League City, TX, Mar. 13-17, 2006.

UCRL-ABS-221280.

Graham, G. A., et al., 2006. "Application of focused ion beam microscopy to planetary and space sciences." Scanning 28, 66. UCRL-ABS-219825.

Graham, G. A., et al., 2006. Experimental impact craters in aluminum foils: insights for cometary sample return. 37th Lunar and Planetary Sciences Conf., League City, TX, Mar. 13-17, 2006. UCRL-ABS-218200.

Graham, G. A., et al., 2006. "Focused ion beam recovery of hypervelocity impact residue in experimental craters on metallic foils." Meteoritics Planet. Sci. 41(2), 159.

UCRL-JRNL-216922.

Huber, H., H. A. Ishii, and S. Brennan, 2006. "Selenium and sulphur distribution in the anomalous CK chondrite EET 99430." Meteoritics Planet. Sci. 41, Suppl. 1, A79.

UCRL-ABS-221287.

Ishii, H. A., and J. P. Bradley, 2006. "Macroscopic subdivision of silica aerogel collectors for sample return missions." Meteoritics Planet. Sci. 41, 233. UCRL-JRNL-215638.

Ishii, H. A., et al., 2006. Micro-SXRF on interplanetary and cometary dust particles: technical considerations for trace element analysis. 37th Lunar and Planetary Sciences Conf., League City, TX, Mar. 13-17, 2006. UCRL-ABS-219148.

Keller, L. P., et al., 2006. Infrared, UV/VIS and Raman spectroscopy of Comet Wild-2 samples returned by the Stardust mission. 37th Lunar and Planetary Sciences Conf., League City, TX, Mar, 13-17, 2006. UCRL-ABS-218252.

Toppani, A., et al., 2006. Segregation of $\mathrm{Mg}, \mathrm{Ca}, \mathrm{Al}$, and Ti in silicates during ion irradiation. 37th Lunar and Planetary Sciences Conf., League City, TX, Mar. 13-17, 2006. UCRL-ABS-218289.

Toppani, A., et al., 2006. Wark-Lovering rims at the nanometer scale: a transmission electron microscopy study. 37th Lunar and Planetary Sciences Conf., League City, TX, Mar. 13-17, 2006. UCRL-ABS-218288.

Zolensky, M., et al., 2006. Mineralogy and petrology of Comet Wild 2 nucleus samples. 37th Lunar and Planetary Sciences Conf., League City, TX, Mar. 13-17, 2006.

UCRL-ABS-221286. 
Zolensky M., et al., 2006. "Mineralogy and petrology of Comet Wild 2 nucleus samplesfinal results of the preliminary examination team." Meteoritics Planet. Sci. 41, Suppl. 1, A167. UCRL-ABS-221291.

\title{
The Chemistry of Core Formation
}

\author{
Frederick J. Ryerson 06-ERI-002
}

\begin{abstract}
Core formation represents the major chemical differentiation event on the terrestrial planets, involving the separation of a metallic liquid from the silicate matrix that subsequently evolves into the current silicate crust and mantle. The generation of the Earth's magnetic field is tied to formation of the core and is important in establishing planetary habitability. The depth and temperature of core segregation controls the chemistry of the core. High-pressure experiments can be used to simulate metal-silicate segregation. In this project, we will develop methods that link a diamond anvil cell (DAC) with analytical systems such as a secondary-ion mass spectrometer with nanometer-scale spatial resolution, a focused ion beam system, and a transmission electron microscope, to determine the partitioning of elements such as sulphur, silicon, oxygen, carbon, hydrogen, vanadium, tungsten, molybdenum, ruthenium, and palladium at extreme conditions relevant to the Earth's lower mantle.

Results of this research will help to explain the low density of the Earth's core relative to pure iron and the overabundance of siderophile elements-chemical elements that partition strongly into a metal-rich phase-in the silicate mantle. This explanation will constrain the potential range of core-forming processes on the Earth, which ultimately constrains generation of the Earth's magnetic field. Constraining composition of the Earth's core may provide clues to the absence of magnetic fields on other terrestrial planets and the influence of magnetic fields on planetary habitability.
\end{abstract}

\section{Mission Relevance}

The extreme conditions of temperature and pressure present in the inner working of the Earth are the same as those in the stars and in nuclear weapons. The microanalytical methods, materials characterization techniques, microanalysis, and high-pressure experimentation that will be developed in this project to understand the Earth's core are applicable to LLNL's stockpile stewardship mission and to forensics efforts for the nonproliferation mission. This project also contributes to the Laboratory's mission in basic science.

\section{FY06 Accomplishments and Results}

In FY06 we (1) installed a new multi-anvil press and tested high-temperature furnace assemblies; (2) developed a method to synthesize, using the Lab's femtosecond laser machining capabilities, $30-\mu \mathrm{m}$-diameter disks of metal-silicate assemblages suitable for 
a laser-heated DAC; (3) used nanoSIMS to measure the partitioning of iron, magnesium, cobalt, and nickel between ferropericlase and perovksite synthesized in the laser-heated DAC; (4) observed the effects of the high-spin-low-spin transition of iron, thus confirming predictions based on x-ray absorption spectrometry; and (5) demonstrated a transition in the partitioning of rare earth elements among the phases produced in mid-ocean ridge compositions at lower mantle conditions, simulating the subduction of oceanic crust.

\title{
Proposed Work for FY07
}

We will focus on extending the pressure and temperature range of siderophile element partitioning experiments between silicates and metal using the piston cylinder, multianvil, and laser-heated DAC methods. A key aspect of the investigation will be fabrication of metal-silicate starting materials with submicron grain sizes to ensure that the region heated in the laser-heated DAC covers a chemically homogeneous area. In addition, we will investigate the solubility of carbon in metallic melts, placing a limit on the incorporation of this light element in the core.

\section{Evidence for Stratospheric Downwelling Associated with High-Elevation Topography}

\author{
Robert C. Finkel 06-ERI-005
}

\begin{abstract}
The continued presence of chlorine-36 $\left({ }^{36} \mathrm{Cl}\right)$ in streams in the Sierra Nevada is thought to be residual nuclear fallout, but the required fallout reserve implies groundwater storage that contradicts current hydrologic models. Here we test the hypothesis that the source of the unexpectedly high concentration of nuclides in the Sierra Nevada is downwelling from the stratosphere during stratocumulus storms. We will measure beryllium-7 ( $\left.{ }^{7} \mathrm{Be}\right)$, ${ }^{10} \mathrm{Be}$, and ${ }^{36} \mathrm{Cl}$ in precipitation and soil samples from the Sierra Nevada. Because these nuclides are produced in the upper atmosphere, their measured abundance should serve as an indicator of stratospheric input to the hydrologic system. We will collaborate with the U.S. Geological Survey (USGS) and UC Merced to collect these samples. This project will either substantially change the hydrologic models or reveal an unrecognized pathway for stratosphere-troposphere exchange that will have a significant impact on our understanding of atmospheric circulation, chemistry, and regional climate.

The test of the hypothesis is straightforward. If stratospheric downwelling occurs to the extent necessitated by the observed ${ }^{36} \mathrm{Cl}$ levels in Sierra streams, it should be straightforward to detect it by direct measurement of ${ }^{7} \mathrm{Be},{ }^{10} \mathrm{Be}$, and ${ }^{36} \mathrm{Cl}$ in precipitation samples collected from an established array of sampling locations. If elevated levels of these nuclides are not found, it would cast severe doubt on our hypothesis. In this case, Sierra hydrologic models will have to take high levels of groundwater storage into account. If elevated levels of these nuclides are found in Sierra precipitation, it could only be due to stratospheric input, both because nuclear fallout is no longer occurring and ${ }^{7} \mathrm{Be}$ and ${ }^{10} \mathrm{Be}$ are not produced by atmospheric nuclear tests.
\end{abstract}




\title{
Mission Relevance
}

This project supports the Laboratory's national security mission because of its relevance to atmospheric dispersal monitoring for nuclear and other releases. The work also supports the environmental-management mission areas of atmospheric and climate modeling and atmospheric protection from ozone-depleting and greenhouse gases. An ability to characterize certain types of stratosphere-troposphere exchange pathways using relatively inexpensive ground level measurements, as opposed to airborne tropopause measurements, would enhance LLNL's capabilities for testing regional atmospheric models.

\section{FY06 Accomplishments and Results}

After a midyear start, we (1) measured background ${ }^{36} \mathrm{Cl}$ levels in water samples collected from several Yosemite Valley sites in collaboration with UC Merced; (2) collected direct rainfall samples at sites near Tioga Pass; (3) assembled ion-exchange rainwater samplers at LLNL, and determined suitable field sites in the Sierra Nevada for their placement in collaboration with the USGS in Denver; (4) obtained a commercial wet/dry deposition collector that is the standard sampler used by the National Atmospheric Deposition Program, and began planning field siting and sampling; (5) collected soil samples at various high elevation sites in the Sierra Nevada; and (6) began the permitting process for water sampling and placement of ion-exchange resin and wet/dry samplers.

\section{Proposed Work for FY07}

During FY07, we will continue establishing a precipitation- and soil-sampling network in collaboration with the USGS and UC Merced. We will analyze snow samples and an initial suite of late-year soils, potentially containing the stratospheric nuclides ${ }^{7} \mathrm{Be}$ and ${ }^{10} \mathrm{Be}$. Fifteen to twenty unattended rain samplers will be constructed and deployed in the summer, with initial samples to be analyzed by the end of FY07. Stream samples will be collected year-round to monitor the relationship between precipitation input and ${ }^{36} \mathrm{Cl}$ content.

\section{Dielectric Properties of Oil Shale}

\author{
Jeffery J. Roberts 06-FS-008
}

\begin{abstract}
The in situ heating of kerogen in an oil shale deposit is required to convert the kerogen into a crude oil suitable for pumping from the subsurface. This feasibility study will determine whether in situ radio-frequency (RF) energy penetrates oil shale deposits and heats the kerogen in a larger region around the heating source than do diffusive methods. This work is important because of the vast resource of oil shale present in the U.S., and because in situ methods are environmentally favorable over mining and surface retort methods.
\end{abstract}


We propose to measure the complex dielectric properties of oil shale as a function of saturation over a wide range of frequencies (10 MHz to $1 \mathrm{GHz}$ ). Measurements and results will be used to determine (1) skin depth (i.e., penetration of RF energy) as a function of water saturation at a few RF frequencies; (2) how dielectric loss varies with frequency and what the optimal RF frequencies for operation are; and (3) what heating rates can be expected for some appropriate sets of conditions, including oil shale grade, water content, power output, and how these factors evolve with time. This information will permit an assessment of the feasibility of using RF heating in situ for oil production from oil shale.

\title{
Mission Relevance
}

This project supports LLNL's energy security mission by conducting fundamental research on a technology with the potential to provide the nation with an abundant, reliable energy source with a minimum of environmental impact.

\section{FY06 Accomplishments and Results}

Frequency-dependent dielectric measurements between $1 \mathrm{MHz}$ and $1.8 \mathrm{GHz}$ were performed on oil shale from two locations-one in Anvil Points, Colorado, and another in southwestern Wyoming. We measured real and imaginary relative dielectric permittivity, loss tangent, impedance magnitude, and phase as a function of temperature $\left(23-146^{\circ} \mathrm{C}\right)$, saturation (0-100\%), pore fluid conductivity, and fabric. Oil shale grade varied between 30 and 55 gallons per ton. From the measured properties we calculated skin depth and the power that can be delivered to the rock in order to estimate heating rates for specific configurations. Although no unique dielectric loss mechanism was observed in wet or dry shale, losses in wet rock suggested that the in situ recovery of oil by RF heating may be feasible.

\section{Publications}

Roberts, J. J., et al., 2006. Dielectric properties of oil shale. 26th Oil Shale Symp., Golden, CO, Oct. 16-18, 2006. UCRL-ABS-222487.

\section{Thermal Diffusivity and Conductivity Measurements in Diamond Anvil Cells}

Daniele Antonangeli 06-FS-009

\begin{abstract}
We have undertaken a study of the feasibility of an innovative method for determining thermal properties of materials at extreme conditions. We propose to explore an application of the flash method, by modifying it to suit the physical and geometric constraints of the diamond anvil cell (DAC). In these experiments, we will use a femto-
\end{abstract}


second laser pulse to heat the back side of the sample, and measure the profile and arrival time for the temperature rise on the front side of the sample to indicate thermal properties of the material being investigated. Our ultimate goal is to greatly expand the pressure and temperature range over which thermal properties can be investigated and to perform test experiments to establish the technique for probing thermal diffusivity on samples in DACs.

When fully integrated into high-pressure capabilities, this technique will allow experimental investigation of thermal properties in a pressure and temperature range much larger than can presently be achieved. The measurements will lead to at least one publication on the technique itself, and ultimately to new knowledge and other publications when applied to materials of interest to basic science and Laboratory programs.

\section{Mission Relevance}

The proposed project will add to our core competence in static high-pressure and hightemperature experimentation, which supports the Laboratory's mission in stockpile stewardship. If successful, this will be a very useful technique for making thermal properties measurements on a wide range of materials, furthering LLNL's commitment to breakthroughs in fundamental science.

\section{FY06 Accomplishments and Results}

In FY06, we designed and implemented an innovative method of determining the thermal properties of materials at extreme conditions based upon an extension of the flash method. In this technique, we used an ultra-fast pulsed laser ( 160 fs pulse width, $1 \mathrm{kHz}$ repetition rate) as the heating source, making it unnecessary to have direct thermal contact with the specimen. The laser pulse is minimally affected by the presence of diamonds that comprise the DAC. We also implemented a capability to tune the wavelength for optimal coupling of the heating source with different samples. In addition to developing the diamond anvil system, we tested the optics and electronics necessary for quick reading of the thermocouple signal, and began test measurements of thermal diffusivity on samples of dimensions compatible with the DAC. 
Laboratory Directed Research and Development

\section{Energy Supply and Use}




\title{
Persistent Monitoring Platforms
}

\author{
Charles L. Bennett 03-ERD-076
}

\begin{abstract}
In this project, we plan to build and test a model of a stratospheric aircraft powered by thermal energy from the Sun. Such an aircraft could maintain station over a designated ground location almost indefinitely, since it would not need fuel. We are developing a thermally coupled system with an efficiency nearly an order of magnitude better than the state of the art (Helios) by creating the technology for a Sun-tracking solar-heat collector, thermal storage reservoir, and a direct-drive Stirling heat engine. We will also develop physics models for thermal transport, materials interactions, loss mechanisms, and engine performance in the stratosphere's environment.
\end{abstract}

We will develop and validate the physics models to prove the principles involved in a solar thermal-powered aircraft in preparation for constructing a prototype scale-model aircraft to demonstrate station-keeping capability at sea level. Successful demonstration of this technology would set the stage for construction of a stratospheric-altitude prototype capable of circumnavigating the globe.

\section{Mission Relevance}

Persistent surveillance, having essentially unlimited dwell time over a region of interest, would enable the acquisition of a qualitatively new type of intelligence information for various national security applications, such as countering the proliferation of weapons of mass destruction. Furthermore, inexpensive persistent surveillance has direct utility in border monitoring for homeland-security missions.

\section{FY06 Accomplishments and Results}

In FY06, we successfully addressed outstanding issues for solar-powered persistent monitoring aircraft, including (1) how heat is transported from collection to storage with low thermal loss; (2) how lightweight, inexpensive, and durable the collection optics can be made; (3) how the Sun can be tracked with low mass, low power, and adequate accuracy; and (4) what performance parameters are required for practical applications. We ultimately demonstrated that our basic concept is capable of powering a small prototype aircraft with sufficient energy storage to remain aloft overnight. On the basis of this work, a federal agency sponsor will fund further development of the technology. 


\title{
Environmental Consequences of Large-Scale Deployment of New Energy Systems
}

Thomas J. Phillips

05-ERD-047

\begin{abstract}
This project will address critical U.S. energy security and environmental management needs by using a suite of climate simulation tools to assess the feasibility of forest regrowth as a strategy for mitigating potential global climate change that results from increasing levels of atmospheric carbon dioxide. However, the ultimate climatic impacts of forest regrowth are complicated by the fact that either heating or cooling of the land surface may result, depending on where the land-cover changes are implemented. In this study, we will use a hierarchy of existing climate-simulation models of different complexities, and run computer simulations to quantitatively bracket the likely range of possible climatic outcomes to be expected from the proposed mitigation strategy.

We expect that this project will produce detailed evaluations of the potential effects of large-scale implementation of the proposed climate-mitigation strategy of large-scale forestation. These results will benefit future climate-carbon studies and help guide longterm climate-mitigation strategies and energy-technology research.
\end{abstract}

\section{Mission Relevance}

Our proposed work supports Lawrence Livermore's energy security and environmental management missions by applying powerful environmental modeling and assessment tools to evaluate the large-scale feasibility of proposed energy systems and carbonmanagement technologies.

\section{FY06 Accomplishments and Results}

In FY06, we conducted a series of simulations of climate-carbon-cycle effects of largescale forest regrowth in a suite of global climate models of different complexity (i.e., with such land-cover changes either prescribed or dynamically predicted), and we bracketed the range of environmental outcomes to be expected. Results from all model simulations are qualitatively similar: large-scale forestation projects are likely to be beneficial in mitigating future carbon-dioxide-induced global warming if these are carried out in the tropics. However, forest regrowth is predicted to be ineffectual, or even counterproductive, if it is implemented in temperate or higher latitudes. We published several peer-reviewed journal papers and gave numerous scientific presentations on this work.

\section{Publications}

Bala, G., et al., in press. "Combined climate and carbon-cycle effects of large-scale deforestation." Proc. Natl. Acad. Sci. Unit. States Am. UCRL-JRNL-225431. 
Bala, G., et al., 2006. "Biogeophysical effects of $\mathrm{CO}_{2}$ fertilization on global climate." Tellus B 58(5), 620. UCRL-JRNL-213984.

Bala, G., et al., 2006. "Direct physical effects of $\mathrm{CO}_{2}$ fertilization on global climate." UCRL-POST-217229.

Bala, G., et al., 2006. "Multicentury changes to the global climate and carbon cycle: results from a coupled climate and carbon cycle model." J. Clim. 18, 4531. UCRL-JRNL-209851.

Bala, G., et al., 2006. Multicentury changes to the global climate and carbon cycle: results from a coupled climate and carbon cycle model. UCRL-PRES-2132777.

Bala, G., et al., 2006. "Will afforestation strategies help mitigate global warming?" Eos Trans. AGU 87(52), Fall Mtg. Suppl., B53E-03. UCRL-ABS-224194.

Caldeira, K., et al., 2006. "Carbon, biophysics, and climate: Where do forests warm? Where do forests cool?" Eos Trans. AGU 87(52), Fall Mtg. Suppl., GC51A-0431. UCRL-ABS-224349.

Caldeira, K., et al., 2006. Carbon sequestration in forests and at Earth's surface. UCRL-PRES-220494.

Caldeira, K., et al., 2005. "Will growing forests make the global warming problem better or worse?" Eos Trans. AGU 86(52), Fall Mtg. Suppl., B31D-03. UCRL-PRES-219807.

Duan, Q., et al., 2006. Effects of global land-cover changes on hydrologic regime changes. UCRL-PRES-219808.

Gibbard, S., et al., 2006. "Climate effects of global land cover change." Geophys. Res. Lett. 32(23), L23705. UCRL-JRNL-215046.

\section{Separation of Carbon Dioxide from Flue Gas Using Ion Pumping}

Roger D. Aines

$$
\text { 06-ERD-014 }
$$

\section{Abstract}

Cost-effective separation of carbon dioxide $\left(\mathrm{CO}_{2}\right)$ from combustion sources is the main limitation to lowering carbon emissions. In this project, we propose to separate $\mathrm{CO}_{2}$ from flue gas by ionic pumping of carbonate ions dissolved in water. The ion pump dramatically increases dissolved carbonate ion in solution and hence the overlying vapor pressure of $\mathrm{CO}_{2}$ gas, allowing its removal as a pure gas. This novel approach to increasing the concentration of the extracted gas permits new approaches to treating flue gas, because the slightly basic water used as the extraction medium does not dissolve the trace acid gases that destroy existing solvents, and therefore no pre-separation is necessary. We will determine the chemical parameters that govern the method through a combination of computer modeling and laboratory experiments. 
We anticipate that our method will compete favorably with current chemical stripping systems used for $\mathrm{CO}_{2}$ separation at power plants-which incur a 35\% energy penalty-and possibly provide a dramatically improved solution to the problem of removing carbon from hydrocarbon combustion products. Our method can be easily demonstrated on small sources, which will, in turn, help build confidence in the method and quantify operating parameters. If successful, we will be in a position to advance a follow-on proposal for a demonstration at the 10-MW scale.

\section{Mission Relevance}

This project supports the energy-security and environmental-management missions by enhancing currently proposed carbon-management options such as technology development for fuel efficiency via fuel cells, hydrogen fuel, and other methods, as well as fossil-fuel recovery and $\mathrm{CO}_{2}$ sequestration.

\section{FY06 Accomplishments and Results}

We used electrodialysis to treat a solution of potassium phosphate saturated with air and $\mathrm{CO}_{2}$, increasing the ionic strength (total ions in solution) by a factor of ten. The solution was found to rapidly effervesce $\mathrm{CO}_{2}$ during treatment. These results matched our calculations well, indicating that we can confidently use geochemical modeling results to design and interpret future experiments. Our original intent to measure the released gas was hampered by the unexpectedly rapid release of gas throughout the electrodialysis apparatus, so we redesigned the experiments to measure the total carbon in the water phase. We are now prepared to make accurate measurements of optimized carrier fluids and different gas mixtures. We have filed a provisional patent application for this method.

\section{Proposed Work for FY07}

In FY07, we will (1) investigate optimizing the ion pump components, ionic composition of the water, and plate materials; (2) investigate the range of solution compositions that improve efficiency and better enable the separation of other components; (3) use real exhaust and flue gases to test the process; and (4) develop a predictive process model.

\section{Publications}

Aines, R. D., W. L. Bourcier, and M. A. Johnson, 2006. Separation of carbon dioxide from flue gas using ion pumping. 8th Intl. Symp. Greenhouse Gas Technology, Trondheim, Norway, June 19-22, 2006. UCRL-CONF-220812. 
140 LDRD FY2006 Annual Report 
Laboratory Directed Research and Development

\section{Engineering and Manufacturing Processes}




\title{
Acoustic Characterization of Mesoscale Objects
}

Diane J. Chinn

\author{
04-ERD-013
}

\begin{abstract}
We propose to explore the science and engineering required to provide state-of-theart acoustic capabilities for nondestructive characterization of mesoscale objects with micrometer resolution. An acoustic technique is attractive because it offers high sensitivity to features such as thickness and interface quality that are important to mesoscale objects. In addition to the resolution requirements, many mesoscale objects require a technique that avoids contact to prevent damage of fragile surfaces. This research will achieve micrometer-resolution characterization by extending the range of laser-acoustic testing to gigahertz frequencies. We intend to deliver a gigahertz acoustic characterization system that can be used to characterize objects comprised of different materials.
\end{abstract}

We will develop a basic understanding of gigahertz wave propagation and a tool for acoustic characterization of high-energy-density physics and cryogenic target components. The understanding gained by this research will broaden the field of acoustic testing by filling the existing gap in acoustic characterization capabilities-work in this area has never been performed.

\section{Mission Relevance}

This promising technique will provide an acoustic characterization tool for many mesoscale applications. In particular, it will benefit high-energy-density physics experiments in support of Lawrence Livermore's stockpile stewardship mission. Other applications could support the Laboratory's energy-security mission by enabling developments in fuel cells or providing understanding of geochemical processes. Using this technique to study tissue and cell abnormality would support LLNL's mission in bioscience to improve human life.

\section{FY06 Accomplishments and Results}

The primary research goal in this final year was to understand gigahertz acoustic-wave propagation and its potential for material characterization. In FY06, we demonstrated that interface characterization such as diffusion-bonded and glued interfaces, and material microstructure identification such as anisotropy and inhomogeneous grain size, are two areas where gigahertz laser-acoustic testing can have an impact on mesoscale nondestructive evaluation. Two peer-reviewed journal publications detailing our research are currently in progress. This LDRD project enabled experimental and analytical validation of gigahertz acoustic characterization for mesoscale objects. We assembled a prototype gigahertz laser-acoustic system, and experimental data were validated with laser-acoustic models-propagation distances of up to $1 \mathrm{~mm}$ in aluminum were achieved with this system. 


\title{
Understanding and Improving High-Voltage Vacuum Insulators for Microsecond Pulses
}

David A. Goerz

06-ERD-033

\begin{abstract}
Insulators are critical components in high-energy pulsed power systems that are prominent in diverse national security and industrial applications. In these systems, high-voltage vacuum insulators often fail because of a mechanism known as surface flashover. This project intends to improve insulator performance by providing a detailed understanding of the flashover mechanism. Our research will employ both computations and experiments to understand the flashover mechanism, concentrating on geometric effects on flashover strength, surface-defect effects on initiation, and secondary-electron avalanche phenomena.

In addition to providing a better understanding the flashover mechanism, the effects of geometry and defects on initiation, and electron avalanche phenomena, this research will also contribute to a better understanding of possible mitigation schemes based on geometrical and material modifications.
\end{abstract}

\section{Mission Relevance}

Pulsed power is critical for Laboratory efforts in nuclear weapons science, national security, and defense missions. Applications of improved, high-voltage vacuum insulators developed in this project include high-energy-density physics and hydrodynamics experiments, energizing accelerators, radiofrequency and microwave technologies, laser and pulsed neutron sources, interpreting weapons effects, lightning and electromagnetic pulse simulators, radiography machines, inertial fusion drivers, directed energy weapons, and electromagnetic launchers.

\section{FY06 Accomplishments and Results}

In FY06, we concluded the electrostatic modeling in two and three dimensions (2D and 3D) to study the influence of insulator geometry on flashover and sensitivity to defects. Additionally, particle-in-cell (PIC) modeling in 2D and 3D was used to study secondaryelectron avalanche and the influence of geometry and surface properties on flashover. In support of the electrostatic and PIC modeling efforts, we assembled a high-voltage, vacuum-insulator test stand with key diagnostics. We then conducted a set of staged experiments to investigate the initiation of flashover at the triple junctions for selected low-dielectric constant materials such as plastics.

\section{Publications}

Houck, T. L., et al., 2006. Study of vacuum insulator flashover for pulse lengths of multimicroseconds. 2006 Linear Accelerator Conf., Knoxville, TN, Aug. 21-25, 2006.

UCRL-CONF-223361. 


\title{
A Compact, High-Intensity Neutron Source Driven by Pyroelectric Crystals
}

\author{
Jeffrey D. Morse 06-ERD-065
}

\begin{abstract}
The objective of this project is to establish a new paradigm for active neutron-interrogation systems. We will explore the potential for achieving an extremely compact, high-intensity neutron source exploiting nuclear fusion reactions driven by pyroelectric crystals. The concept being investigated represents a revolutionary approach for accelerator-induced nuclear fusion reactions in a compact platform: Pyroelectric-crystal-driven neutron sources would potentially eliminate the need for large, high-voltage power supplies and radically change the size and configuration of the ion accelerator, enabling a palm-sized neutron source. Thus, this project could have broad impact on weapons science, nuclear physics, and homeland security applications.
\end{abstract}

We will quantitatively determine the potential for scaling pyroelectric-crystal-driven ion and neutron sources to fluxes of $10^{6} \mathrm{n} / \mathrm{s}$ or higher. Our technical approach is to (1) complete a modeling study of the crystal-based neutron source; (2) demonstrate experimental scaling -that is, neutron output up to three orders of magnitude greater than the initial results; and (3) test and evaluate the neutron source in actual applications. Successful results will enable new analytical techniques for a range of applications related to national security.

\section{Mission Relevance}

Neutron interrogation provides a noninvasive method of screening cargo and shipping containers for special nuclear materials smuggled through ports, one of the most challenging issues facing the nation's border security. This project supports Lawrence Livermore's national security mission by investigating a promising new technique that may enable a field version or even handheld neutron sources with the ability to interrogate targets anywhere, not just at ports. This approach offers the further possibility of a remote, autonomous neutron probe for the covert interrogation of targets and threat identification.

\section{FY06 Accomplishments and Results}

In FY06 we (1) developed a self-consistent system model for crystal-based neutron sources, (2) conducted design assembly work for a crystal polarization-modulation experiment, (3) modeled and designed ion sources using a three-dimensional electrostatic field solver, and (4) modeled ion trajectory and acceleration driven by a polarizationmodulated-crystal-stack high-voltage source. Meeting all of our milestones, we demonstrated a new field ionization model predicting the charge and voltage buildup on the surface of the nanometer sharpened-metal tips. Our approach provided a physics model bridging the low-field and high-field ionization models in the literature. Combining the field ionization model with a self-consistent system model, the resulting charge, field, and ion current occurring during a thermal ramp of a pyroelectric crystal were modeled. 


\title{
Proposed Work for FY07
}

We will (1) explore polarization modulation limits for pyroelectric crystal materials;

(2) experimentally characterize polarization modulation limits in lithium tantalate $\left(\mathrm{LiTaO}_{3}\right)$;

(3) microfabricate arrays of gated and ungated tips and assemble them on crystal

surfaces; (4) test and evaluate the ion source, and optimize the ion beam current; and

(5) assemble and evaluate the accelerator design using high-voltage probes and an ion spectrometer.

\section{Transport Behavior and Conversion Efficiency in Pillar-Structured Neutron Detectors}

Rebecca J. Nikolic

06-ERD-067

\begin{abstract}
A radiation detection device that can be easily fielded and offers high detection efficiency is vital to national security efforts. In this project, we will demonstrate technology that could lead to a device having a thermal neutron detection efficiency of over $70 \%$. By applying microtechnology methods to neutron detection, we expect to make revolutionary improvements in device efficiency and field usability. We will take advantage of recent advancements in material science, charged carrier transport, and neutron-to-alpha conversion dynamics to fabricate semiconductor pillars in a three-dimensional matrix in which the neutron-to-alpha conversion material has adequate density to capture the full neutron flux.
\end{abstract}

We anticipate achieving a detection efficiency of $30 \%$ in a demonstration device, and will devise a roadmap for scaling the device to optimal efficiency. This is significant because current technology suffers from poor efficiency and adaptability to field use, high voltage, sensitivity to microphonics, a large device footprint, and high pressure, resulting in significant complications in air transport and deployments. The advances we propose in micro- and nano-fabrication methods are applicable to many other fields, including biochemical detection, communications, and computations.

\section{Mission Relevance}

Our project supports the Laboratory's national security mission by advancing technology for the detection of special nuclear materials and radiological dispersal devices. If our device meets the requirements for high efficiency and demonstrates suitability for field use, it would pave the way for manufacturing field-ready devices in partnership with an industrial collaborator.

\section{FY06 Accomplishments and Results}

In FY06, we (1) began characterizing a 5- $\mu \mathrm{m}$ pillar detector; (2) investigated borondeposition dynamics and developed a boron-deposition technique based on the absorption and decomposition of chemical vapors and capable of a greater-than-50\% fill factor 
between pillars; and (3) fabricated and assembled a small-footprint, multiple-wire, gasproportional counter to determine the conversion efficiency of boron films.

\section{Proposed Work for FY07}

In FY07, we will (1) develop methods to deposit boron materials in the pillar matrix with a target fill factor of $80 \%$; (2) investigate neutron-to-alpha conversion efficiency in highresistivity, vapor-deposited boron films; (3) study electron and hole transport in vertical high-aspect-ratio features, analyze leakage paths, and work to minimize component size; (4) model a semiconductor for high-aspect-ratio diodes; and (5) determine the radiation spectra of fabricated pillar chips. 
Laboratory Directed Research and Development

\section{Materials Science and Jechnology}




\title{
A Two-Particle Formulation of Electronic Structure
}

\author{
Antonios Gonis \\ 03-ERD-064
}

\begin{abstract}
The problem of correlated electrons in elemental solids and alloys figures prominently in the Laboratory's efforts to understand the behavior of plutonium metal and its alloys and to predict such properties as phase and dimensional stability under self-irradiation and as a function of time. We have introduced theoretical and computational methods for studying the electronic structure of matter based on two-particle states (and, in general, $n$-particle states) rather than single-particle states as is current practice. The codes we develop will thus simulate the Coulomb interaction and its effect on materials properties with greater accuracy and provide deeper insight into the physics of materials than is possible with traditional methodology.

This work could provide theoretical and practical electronic structural information about important materials and systems for which conventional methods are currently inadequate, particularly complex systems such as plutonium. In the long term, we will develop realistic models for the study of heavy elements and their alloys and apply them to materials central to the Laboratory's mission. The methodology developed will not only allow the simulation of materials properties but also provide insight into the basic mechanisms driving these properties.
\end{abstract}

\section{Mission Relevance}

Understanding the electronic structure and properties of materials, especially actinides, is crucial to nuclear science and relevant to areas such as stockpile stewardship, in support of the Laboratory's national security mission.

\section{FY06 Accomplishments and Results}

In FY06, we (1) advanced our methodology for studying electronic structure, (2) completed the formalism for practical application of our approach to electronic structure calculations, and (3) completed model calculations on linear systems within the Hubbard model for various values of the relevant parameters, showing the viability of our method in calculating spectral properties in the presence of the Coulomb interaction. 


\title{
Plutonium and Quantum Criticality
}

\author{
Michael J. Fluss \\ 03-ERD-077
}

\begin{abstract}
Understanding the anomalous properties of plutonium that arise because the $5 f$ electrons are poised between localized and itinerant behavior is a science challenge with important technological implications. By exploring the possibility of a second-order phase transformation at a temperature of $0 \mathrm{~K}$, a quantum critical point $(\mathrm{QCP})$, we hope to explain the origin of plutonium's complexity. We will "tune" alpha- and delta-plutonium using disorder, alloying, pressure, and magnetic fields while characterizing the physical properties at low temperature. This will accomplish our primary goal of identifying the $\mathrm{QCP}$; we will further characterize the physical properties of plutonium in the vicinity of the $\mathrm{QCP}$, thus mapping its low-temperature phase diagram.
\end{abstract}

Our goal is to identify a hypothesized QCP, which can be identified by a singularity in certain physical properties such as specific heat at temperatures of $0 \mathrm{~K}$. Mapping the lowtemperature phase diagram for plutonium will provide high-fidelity measurements of its physical properties, which are needed to validate theories on how to correctly introduce many-body effects into the first-principles description of plutonium and plutonium alloys. We also will develop evidence for the existence or absence of local moments in the plutonium system through experiments with point defects and muon relaxation. This work will provide new insight into the organizing principles of plutonium and a more fundamental understanding of the origin of its six solid-state phases and its many anomalous physical properties, including its large volume changes and extraordinary phase sensitivity to impurities.

\section{Mission Relevance}

By contributing to a better understanding of the physical properties of plutonium, this project explores the foundational science of stockpile stewardship. In addition, this research supports Lawrence Livermore's mission in breakthrough science and technology.

\section{FY06 Accomplishments and Results}

In FY06, by following the experimental clues established in the previous two years, we chose to examine the magnetic and superconducting properties of plutonium-americium, and found that trace amounts of superconductivity are present in the plutonium-22-at.\% americium system. Additionally, we discovered a "giant" anti-ferromagnetic resonance associated with defect relaxation in this same system. These results are consistent with the hypothesis that plutonium can be chemically tuned to a QCP by expanding the plutonium lattice spacing with americium atoms, which are oversized with respect to plutonium. While we have not yet fully mapped the low-temperature phase diagram in the vicinity of this hypothesized criticality, the project has provided significant evidence and important experimental clues of where this singularity can be found. Overall, this project enabled us to discover that the introduction of disorder into the alpha-plutonium and 
delta-plutonium-gallium systems causes magnetism to emerge, which we interpret as a direct consequence of a nearby criticality.

\section{Publications}

Caturla, M. J., et al., 2006. "Kinetic Monte Carlo simulations applied to irradiated materials: the effect of cascade damage in defect nucleation and growth." J. Nucl. Mater. 351, 78. UCRL-JRNL-220000.

Chapline, G. F., M. J. Fluss, and S. K. McCall, in press. "Plutonium and quantum criticality." J. Alloys Comp. UCRL-JRNL-222222.

Chung, B. W., et al., 2005. "Spectroscopic signature of aging in delta-Pu(Ga)." J. Phys. Soc. Japan 75(5), 54. UCRL-JRNL-217764.

Heffner, R. H., et al., 2006. "Limits for ordered magnetism in Pu from muon spin rotation spectroscopy." Phys. Rev. B. 73(9), 94. UCRL-JRNL-225555.

Heffner, R. H., et al., 2006. "New limits on the ordered moments in alpha Pu and Ga stabilized Pu." Physica B-Condensed Matter. 374, 163. UCRL-JRNL-216609.

Heffner, R. H., et al., in press. "The search for magnetic order in delta-Pu metal using muon spin relaxation." J. Alloys Comp. UCRL-PROC-225444.

Maple, M. B., et al., 2006. "Field-dependent ordered phases and Kondo phenomena in the filled skutterudite compound $\mathrm{PrOs}_{4} \mathrm{As}_{12}$." Proc. Natl. Acad. Sci. Unit. States Am. 103(18), 6783. UCRL-JRNL-224444.

McCall, S. K., et al., in press. "Emergent magnetic moments produced by self-damage in plutonium." Proc. Natl. Acad. Sci. Unit. States Am. UCRL-JRNL-223284.

McCall, S. K., et al., 2005. "Emerging magnetism arising from self-damage in alpha- and delta-plutonium." Actinides 2005-Basic Science, Applications and Technology. Vol. 893, art. no. 0893-JJ04-03. UCRL-JRNL-217247.

McCall, S. K., et al., in press. Isochronal annealing studies in Pu and Pu alloys using magnetic susceptibility. J. Alloys Comp. UCRL-PROC-225490.

McCall, S. K., et al., 2006. "Magnetic properties of radiation damage in Pu and Pu alloys." Royal Society of Chemistry: Recent Advances in Actinide Science 2006. Vol. 305, p. 734. UCRL-PROC-211062.

Morris, G. D., et al. 2006. "Muon spin resonance studies of the superconducting order parameter in PuCoGa ${ }_{5}$ " Phys. B Condens. Matter 374, 180. UCRL-JRNL-216610.

Yuhasz, W. M., et al., 2006. "Multiple ordered phases in the filled skutterudite compound PrOs $_{4} \mathrm{As}_{12}$ " Phys. Rev. B. 73, 144409. UCRL-JRNL-220074. 


\title{
High-Strain-Rate Deformation of Nanocrystalline Metals
}

\author{
Eduardo M. Bringa 04-ERD-021
}

\begin{abstract}
In this project, we will perform experiments and simulations to establish constitutive models for plastic behavior and to determine the deformation mechanism of nanocrystalline materials at different grain sizes $(<100 \mathrm{~nm})$ and high strain rates $\left(>10^{6} / \mathrm{s}\right)$. The experiments will use both laser-induced shocks and isentropic compression to study, for the first time, the high-strain-rate deformation of nanocrystalline nickel. Samples will be characterized before and after loading using transmission electron microscopy, nanoindentation, profilometry, and x-ray diffraction. We will validate constitutive models using both atomistic molecular dynamics and continuum simulations, which although at the limit of current capabilities, will match experimentally accessible length and time scales.
\end{abstract}

Improved understanding of the deformation mechanisms of nanocrystalline metals could lead to the design of improved materials with higher or lower resistance to plastic deformation. Nanocrystals are useful materials for laser targets and other applications because of their high hardness. Our simulations and experiments suggest a novel way to obtain even harder nanocrystals, both during and after shock loading. The results to date are unique because these strain rates had never before been experimentally attained in nanocrystals, and because experiments and atomistic simulations cover the same length and time scales. Our use of local facilities-such as the Janus laser, electron microscopy and characterization facilities, and massively parallel computers-is increasing in-house expertise.

\section{Mission Relevance}

This work will (1) validate constitutive models (such as grain size and grain boundary corrections) important to the Stockpile Stewardship Program, (2) enhance our understanding of nanocrystalline metals important to stockpile stewardship, (3) map deformation processes at high strain rates, and (4) develop massively parallel simulation capabilities to study these processes. In addition, this project will contribute to the Laboratory's mission in basic science.

\section{FY06 Accomplishments and Results}

We accomplished the FY06 planned milestones of (1) loading, transmission electron microscopy, and hardness measurements of new samples; (2) positron measurements of porosity; (3) atomistic simulations of grain boundary sliding under pressure as well as samples with 50-nm grain size; and (4) implementation of a new model of deformation into a continuum (micromechanics) code. During the course of this project we published results in high-visibility publications, gave several invited presentations, and received press coverage of our results around the world. The deformation map that we obtained, together with our new continuum-level model of grain boundary sliding fit to atomistic 
data, can be used to improve current continuum simulations and to plan future experiments involving polycrystals.

\section{Publications}

Bringa, E. M., E. Leveugle, and A. Caro, 2006. "Pressure effects on grain boundary plasticity in nanophase metals." Appl. Phys. Lett. 89, 023101. UCRL-JRNL-330281.

Lebensohn, R., E. M. Bringa, and A. Caro, in press. "A viscoplastic micromechanical model for the yield strength of nanocrystalline materials." Acta Materialia. UCRL-JRNL-219993.

Wang, Y., et al., 2006. Deformation of nanocrystalline materials at ultrahigh strain rates. DyMat 2006, Dijon, France, Sept. 11-15, 2006. UCRL-PRES-224309.

Wang, Y. M., et al., 2006. "Deformation of nanocrystalline materials at ultrahigh strain rates-microstructure perspective in nanocrystalline nickel." J. Phys. IV (France) 134, 915. UCRL-PROC-220628.

Wang, Y. M., et al., 2006. "Deforming nanocrystalline nickel at ultrahigh strain rates." Appl. Phys. Lett. 88, 061917. UCRL-JRNL-213195.

\section{Nanosecond Ultrasonics to Study Phase Transitions in Solid and Liquid Systems at High Pressure and Temperature}

\section{Brian P. Bonner}

$$
\text { 04-ERD-033 }
$$

\footnotetext{
Abstract

In this project, we seek to combine ultrasonic and solid-medium technology to make precise measurements of elastic moduli, attenuation, and dispersion at pressures up to $40 \mathrm{GPa}$ and temperatures up to $2200 \mathrm{~K}$, on samples up to $10 \mathrm{~mm}^{3}$. We will improve equation-of-state (EOS) descriptions by incorporating recent innovations in high-frequency, contact ultrasonics and wave-propagation analysis methods, which were developed for geophysics to study materials with large grains and fabrication-dependent textures, as well as study time-dependent behavior in melts and phase transitions. We are adapting and improving unique ultrasonic methods developed by our university collaborators, and using these methods in our solid-medium, high-pressure apparatus for polycrystalline and liquid samples.
}

We expect to develop a capability for studying time-dependent material properties under extreme conditions to enable, for the first time, direct determination of both the viscoelastic properties of liquids and crystalline mush, and the kinetic phase boundaries of materials. We will be able to measure precisely, at high pressure and temperature, the properties of polycrystalline materials with grain sizes larger than a few hundred micrometers and to make high-temperature and high-pressure measurements of strengthrelated properties (e.g., frequency-dependent shear modulus) to test EOS assumptions. 
The results of this project will advance existing expertise in static high-pressure methods for material characterization, problems in stockpile stewardship, and earth sciences.

\title{
Mission Relevance
}

This work supports Lawrence Livermore's stockpile stewardship mission by providing improved adiabatic data, such as precise velocity measurements for EOS and shear wave data for strength estimates, to improve computer simulations of explosion phenomena. This work also contributes to Laboratory's mission in scientific breakthroughs with highpressure and high-temperature ultrasonic measurements that address important questions about the composition and thermodynamic state of the Earth and other planetary bodies.

\section{FY06 Accomplishments and Results}

In FY06, we used our ultrasonic system to make successful measurements in a unique moissanite anvil cell for cubic millimetre samples, as well as in a 30-GPa piston cylinder apparatus. We performed experiments for (1) water-ice VI across the phase boundary, (2) tin in the vicinity of the alpha-to-beta phase transition at high pressure, and (3) cerium across the gamma-alpha phase boundary. The volume collapse in cerium in the vicinity of $0.7 \mathrm{GPa}$ causes strong effects on ultrasonic propagation. The ultrasonic propagation detected a kinetically controlled mixed-phase region, which suggests an effect of stress shielding on the cerium transition. Overall, we designed and constructed a unique ultrasonic system optimized for high pressure and temperature applications and demonstrated a bandwidth of $400 \mathrm{MHz}$. We made successful high pressure and temperature ultrasonic measurements in four apparatus configurations. In addition, we adapted the E3D elastic wave propagation code to study our experimental configurations and interpret results.

\section{Multiscale Characterization of Body-Centered-Cubic Crystals Deformed to Large Extents of Strain}

Jeffrey N. Florando

04-ERD-036

\begin{abstract}
The objectives of this proposal are to provide large-strain data on body-centered-cubic (bcc) crystals for the validation of dislocation dynamics simulations and the development of crystal plasticity models. The accuracy of such simulations is based on the ability of the underlying theory to capture the necessary physics of deformation, which is currently not well understood for bcc single crystals. Because most materials of interest are deformed to large strains, the need exists to develop new experimental techniques to measure the deformation behavior of materials to large extents of strain. The new experimental design will include using a non-contact method to measure strains, in conjunction with a stress analysis of the deformed sample and multiscale characterization.
\end{abstract}


The insight gained from the results of the large-strain experiments has the potential to impact crystal plasticity theory and advance predictive modeling capabilities. Crystal plasticity models are an intricate part of a multiscale modeling of materials, in which information is passed between simulations conducted at different length scales, with the eventual goal of predicting the deformation response of complex materials under various loading conditions.

\section{Mission Relevance}

Material-strength models that are accurate under extreme conditions of high pressure, high strain rate, and large extents of strain are a major component of the Stockpile Stewardship Program. Such models will also play a role in the simulation of strength experiments at future fusion-class lasers. Little work has been done to develop large-strain models, and computer code simulations require experimental data to develop and validate the multiscale crystal plasticity models currently used.

\section{FY06 Accomplishments and Results}

We experimentally investigated and characterized copper single crystals, achieving surprising results that cannot be explained by current theories. Although not part of the original research plan, these experiments on copper crystals yielded results that could elucidate the fundamental mechanism behind the behavior of bcc crystals. Such knowledge can also be incorporated into existing models. We also wrote and submitted a journal paper-which has been accepted for publication-on our method of using imagecorrelation data to calculate the activity on individual slip systems in deformed single crystals. In summary, this project has developed large-strain experiments and analysis methods, and compared the results with those of simulations.

\section{Publications}

Bulatov, V. V., et al., 2006. "Dislocation multi-junctions." Nature 440, 1174. UCRL-JRNL-212199.

Florando, J. N., et al., in press. "Calculation of slip system activity in deformed zinc single crystals using digital 3-D image correlation data." Phil. Mag. Lett. UCRL-JRNL-219533.

Lassila, D. H., J. N. Florando, and M. M. LeBlanc, 2006. "Zinc single crystal deformation experiment using a '6-degrees-of-freedom' apparatus." Metal. Mat. Trans. UCRL-JRNL-221336.

LeBlanc, M. M., et al., 2006. "Image correlation applied to single crystal plasticity experiments and comparison to stain gage data." Exp. Tech. 30, 33-37. UCRL-JRNL-213561. 


\title{
NanoBIS Determination of the Unoccupied Electronic Structure of Plutonium
}

James G. Tobin

04-ERD-105

\begin{abstract}
The unoccupied conduction-band electronic structure is the missing link in experimental validation of the equation of state for plutonium. This project will employ nanofocused bremsstrahlung-isochromat spectroscopy (nanoBIS) to obtain direct measurements of the conduction band of plutonium, other actinides, and rare earths. By using a scanning electron microscope as the electron beam source in nanoBIS, we will probe singlecrystalline microcrystallites in polycrystalline samples. Furthermore, by working at higher energies, we will sample preferentially for bulk properties, thus reducing the impact of surface effects. Studies will begin by using rare earths such as cerium, an analogue of plutonium with a similar multiphase electronic structure, and the less-radioactive actinides such as depleted uranium.
\end{abstract}

We expect to determine the unoccupied conduction-band electronic structure of plutonium, other actinides, and rare earths in a phase-specific fashion, with emphasis on bulk contributions. Results of this project will resolve the $4 \mathrm{f}$ and $5 \mathrm{f}$ electronic structure controversies by comparing our data directly to the predictions made with dynamical mean-field theory.

\section{Mission Relevance}

In this project, we will validate plutonium equation-of-state theories and provide experimental benchmarking of modeling for science-based stockpile stewardship, in support of the national security mission.

\section{FY06 Accomplishments and Results}

We scaled back the scope of this project, including the nano-component, because of budget reductions. For FY06, we adopted a new goal of pursuing bremstrahlung isochromat spectroscopy without nano-focusing. A major setback was incurred when the delivery of the monochromator system (XES-350) was delayed to late spring 2006. However, during the summer of 2006, we tested the XES-350 system on site under vacuum, with successful collection of high-resolution fluorescence spectra of the zinc $2 p$ doublet near $1000 \mathrm{eV}$ and the oxygen 1s singlet near $500 \mathrm{eV}$. 


\title{
Publications
}

Butterfield, M. T., et al., 2005. "Utilizing nano-focussed bremstrahlung isochromat spectroscopy (nBIS) to determine the unoccupied electronic structure of Pu." Mater. Res. Soc. Symp. Proc. 893, 95. UCRL-PROC-216960.

Tobin, J. G., 2005. Using nano-focused bremstrahlung isochromat spectroscopy (nBIS) to determine the unoccupied electronic structure of Pu. Commissariat à I'Énergie AtomiqueNational Nuclear Security Administration Workshop, Paris, France, Apr. 19, 2005. UCRL-PRES-211497.

Tobin, J. G., in press. "Using nano-focused bremstrahlung isochromat spectroscopy (nBIS) to determine the unoccupied electronic structure of Pu." Recent Advances in Actinide Science, Springer, Berlin, Germany. UCRL-ABS-208354.

Tobin, J. G., 2005. Utilizing nano-focused bremstrahlung isochromat spectroscopy (nBIS) to determine the unoccupied electronic structure of Pu. 5th Intl. Workshop: Fundamental Plutonium Properties, Snezhinsk, Russia, Sept. 12-16, 2005. UCRL-ABS-212808.

Tobin, J. G., et al., 2005. "Experimental benchmarking of Pu electronic structure." Mater. Res. Soc. Symp. Proc. 893, 79. UCRL-PROC-216219.

\section{The Structure and Properties of Nanoporous Materials}

\author{
Anthony W. Van buuren 05-ERD-003
}

\begin{abstract}
Our goal is to quantify the microstructure of highly porous materials, and to determine how material processing relates to the structure and ultimately to mechanical behavior. We will measure how the structure of porous metal and metal-oxide foams changes with temperature, pressure, and surface environment. We will then quantify the structural changes with a combination of small angle $x$-ray scattering (SAXS) and high-resolution, $x$ ray tomography. Finite-element modeling, using the determined structures, will be used to study the effects of mechanical loading on the cell structures, and to map out relationships between processing, density, and strength.

We expect to develop critical technologies for characterizing a new class of high-energylaser target materials. A key deliverable will be the ability to predict the mechanical properties of nanoporous materials and characterize gradient-density foam microstructures for future laser targets. The ultimate aim of the proposed research is to determine how the structure of highly porous metal and metal-oxide foams changes with temperature, pressure, and surface environment, and how changes in the nanostructure of these foams alter their mechanical behavior.
\end{abstract}




\title{
Mission Relevance
}

The ability to characterize and model nanoporous materials developed in this project will further applications in stockpile stewardship and high-energy-density science, in support of the national security mission.

\section{FY06 Accomplishments and Results}

In FY06 we met all of our milestones. We performed SAXS on a number of low-density transition metal-oxide foams, and the data show that there are significant changes to the cell structure as a function of density in the tantalum-oxide aerogel foam. These results lead to a model for the tantalum-oxide aerogel in which $85 \%$ of the mass is confined to the nodes, consistent with the formation of fractal clusters and explaining why these materials are weaker than predicted. Our results provided the first experimental support for a diffusion-limited cluster aggregation model for aerogel growth. In situ ultra-SAXS (USAXS) measurements on tantalum-oxide aerogel at $77 \mathrm{~K}$ showed a decrease in the correlation length of the pore network, which is consistent with an increase in density because of network structure shrinkage.

\section{Proposed Work for FY07}

In FY07, we plan to perform USXAS measurements to understand the effect of synthesis on structure change-in particular, how synthesis conditions can affect the amount of mass at the nodal points in the aerogel. USAXS measurements will be conducted on aerogels at temperatures where nitrogen gas will condense in the aerogel matrix. Knowledge of how cryogenic fluid wets the aerogel is unknown, but it is necessary information if these foams are to be used in laser targets. The USAXS results will provide the correlation length and thickness of the pore wall as a function of density and processing parameters. From these measurements, stochastic lattices will be generated for finite-element simulations, which will then provide elastic constants of the material and be used to study thermoelastic deformation.

\section{Publications}

Kucheyev, S. O., et al., 2006. "Structure of low-density nanoporous dielectrics revealed by low-vacuum electron." Langmuir 10, 1021. UCRL-JRNL-221923.

\section{Characterization and Control of Laser-Induced Modifications in KDP and DKDP Crystals}

\author{
Stavros G. Demos 105-ERD-016
}

\begin{abstract}
Potassium dihydrogen phosphate (KDP) and deuterated KDP (DKDP) crystals are the only materials available for use as frequency converters from fundamental $(1 \omega)$ to
\end{abstract}


second or third harmonics ( $2 \omega$ or $3 \omega$ ) in large-aperture lasers. The interaction of laser light with defect structures in KDP and DKDP crystals can lead either to damage or to their modification into less-absorbing species. The precise conditions controlling these behaviors are not currently known. The objective of this project is to reveal fundamental mechanisms involved in the laser-induced defect reactions leading to bulk damage and laser conditioning in KDP and DKDP crystals. Our ultimate goal is to learn how to control these reactions.

We will significantly enhance our fundamental understanding of the physics involved in laser-induced bulk damage and laser conditioning in KDP and DKDP crystals. This will be accomplished using new diagnostic tools and methods to investigate the microscopic response of a variety of well-characterized materials with variations in relevant physical parameters-including pinpoint size and density versus fluence, wavelength, and pulse length at $2 \omega, 3 \omega$, and combinations thereof, as well as conditioning effectiveness as a function of laser parameters. This work will also extend current knowledge regarding the interaction of high-power laser light with large-bandgap materials.

\section{Mission Relevance}

This project supports LLNL's stockpile stewardship mission by providing novel diagnostic tools to quantify and predict the damage performance of optical materials for largeaperture laser systems, and by offering basic knowledge to optimize conditioning protocols for frequency-doubling and frequency-tripling crystals. In the long term, this project will lead to novel technologies for materials characterization on future large-scale lasers.

\section{FY06 Accomplishments and Results}

We (1) completed our experimental study to reveal the damage-initiation and laserconditioning behaviors of damage precursors in KDP as a function of laser parameters; (2) used this comprehensive set of experimental results to develop a preliminary hypothesis regarding the processes involved; (3) designed additional experiments to test these concepts, showing that specific crystal growth conditions adversely affect the performance of the material; (4) performed experiments to test whether the host (pure) material becomes an absorber under conditions believed to be present in the material surrounding the damage precursor during nanosecond laser irradiation; and (5) published several manuscripts based on our work.

\section{Proposed Work for FY07}

In FY07, we will (1) examine methods to produce a suite of crystals with different damage characteristics by varying laser-conditioning exposure, growth conditions, and irradiation with $\mathrm{x}$ rays or high-energy particles; (2) utilize the differences in the damage precursor populations in selected samples to identify the precursors using conventional characterization techniques; (3) continue to develop detailed models explaining damage initiation and conditioning; and (4) perform a small number of targeted experiments to confirm predictions and resolve remaining important issues. 


\section{Publications}

DeMange, P., et al., 2006. "Different precursor populations revealed by microscopic studies of bulk damage in KDP and DKDP crystals." Proc. SPIE 5991, 59911X. UCRL-ABS-212436.

DeMange, P., et al., 2006. "Differentiation of defect populations responsible for bulk laserinduced damage in potassium dihydrogen phosphate crystals." Opt. Eng. 45, 104205. UCRL-JRNL-217769.

DeMange, P., et al., 2006. "Laser-induced defect reactions governing damage initiation in DKDP crystals." Optic. Express 14, 5313. UCRL-JRNL-220463.

DeMange, P., et al., 2006. "A multi-dimensional investigation of laser conditioning in KDP and DKDP crystals." Proc. SPIE 5991, 599107. UCRL-CONF-216863.

DeMange, P., et al., 2006. "Laser-induced defect reactions governing damage performance in KDP and DKDP crystals." Proc. SPIE 6103, 610305. UCRL-ABS-213588.

DeMange, P., et al., 2006. "Understanding and predicting the damage performance of $\mathrm{KD}_{\mathrm{x}} \mathrm{H}_{2-\mathrm{x}} \mathrm{PO}_{4}$ crystals under simultaneous exposure to 532- and 355-nm pulses." Appl. Phys. Lett. 89, 181922. UCRL-JRNL-214882.

Liu, C. S., et al., 2006. "Electronic structure calculations of an oxygen vacancy in $\mathrm{KH}_{2} \mathrm{PO}_{4}$." Phys. Rev. B 72, 134110. UCRL-JRNL-210039.

Negres, R. A., 2006. "Laser-induced damage in DKDP crystals under simultaneous exposure to laser harmonics." Proc. SPIE 5991, 59911S. UCRL-PROC-216804.

Negres, R. A., et al., 2006. "Expedited laser damage profiling of $\mathrm{KD}_{\mathrm{x}} \mathrm{H}_{2-\mathrm{x}} \mathrm{PO}_{4}$ with respect to crystal growth parameters." Optic. Lett. 31, 3110. UCRL-JRNL-220921.

\section{Ceramic Laser Materials}

Thomas F. Soules

$$
\text { 05-ERD-037 }
$$

\footnotetext{
Abstract

Our objective is to evaluate the use of transparent ceramics for optical components of high-power lasers. Ceramics created by sintering powders at temperatures below their melting point can offer the same optical and thermal properties as single crystals but have advantages such as design flexibility, larger aperture capability, ease and robustness of manufacture, potential multifunctionality, higher and more uniform activator concentrations, and reduced brittleness. We plan to accomplish our objectives by developing a robust process for making transparent ceramics and collaborating with an industrial partner to develop novel, mulitfunctional transparent ceramic parts for highpowered lasers.
} 
In this project, process innovations such as novel methods for preparing the very pure starting nano-powders and methods of extrusion for forming the pre-sintered material will be explored with our in-house synthesis. We will further implement transparent ceramic slabs from our industrial partner, where applicable, and design several laser architectures that are possible only with ceramic materials-examples include large-aperture slabs for a solid-state heat-capacity laser (SSHCL) and monolithic slabs (including the laser gain media) surrounded by a transparent ceramic frame designed to absorb amplified spontaneous emission (ASE).

\section{Mission Relevance}

This project supports the national security mission by providing ceramic laser-gain slab technology for SSHCLs, which can provide the power ( $\sim 30 \mathrm{~kW})$ needed for defense applications such as destroying mortar shells, rockets, and land mines. Current singlecrystal slabs are very difficult to grow, and larger slabs may not be possible. The larger sizes enabled by ceramic slabs would increase available laser power for these applications.

\section{FY06 Accomplishments and Results}

In FY06, we (1) optimized and validated our process for making transparent yttriumaluminum-garnet (YAG) ceramics and significantly improved the optical quality by adding a hot isostatic press step; (2) developed near transparency in several other materials, including strontium fluoride; (3) developed a method to extrude and sinter different shapes; (4) obtained 12,10 - by 10 - by 2 -cm neodymium-doped YAG slabs;

(5) developed epoxy-clad, ASE suppression methods for ceramic amplifier slabs;

(6) fabricated samarium-doped YAG as an ASE suppressor integrally sintered in a frame around the neodymium-doped YAG ceramic; (7) damage-tested ceramic YAG; and (8) measured the spectra and lifetimes of ytterbium-yttrium oxide, a potential high-power laser-gain material.

\section{Proposed Work for FY07}

Our objectives for FY07 are to: (1) develop transparent ceramics of new materials, such as yttrium-strontium fluoride, which can be used as a storage laser with laser diode pumping; (2) develop slip casting and extrusion techniques for fabricating preformed shapes and multifunctional parts; (3) develop new nano-particle technology, including flame pyrolysis, for making precursor materials; (4) develop a new class of very hard alumina-based glasses that could provide very large-aperture lasing media for nextgeneration fusion-class lasers; and (5) continue scale-up efforts with our industrial collaborators and obtain ceramic ytterbium-yttrium oxide for spectroscopy and damage tests. 


\title{
Publications
}

Soules, T. F., 2006. "Transparent ceramics spark laser advances." Science and Technology Review, April 2006, pp. 10-19. UCRL-TR-52000-06-4.

Soules, T. F., et al., 2005. 8th Ann. Directed Energy Symp., Lihue, HI, Nov. 14-18, 2005. UCRL-POST-216750.

\section{Determining the High-Pressure Melting Curve of Iron}

\author{
Jonathan C. Crowhurst 05-ERD-039
}

\begin{abstract}
The original aim of this project was to determine, with unprecedented precision, the melting curve of iron at geophysically relevant pressures. In the course of developing requisite technology and techniques, we encountered novel chemical reactions whose products-nitrides of the noble metals platinum and iridium-are stable or metastable under ambient conditions. We believe the scientific impact of continuing this work will be greater than that of our original goal. For the final year of this project, therefore, we propose to reduce the scope of iron melting and to make our primary focus the chemistry of other platinum-group metals with nitrogen under extreme conditions.

The major impact of this work will be the elucidation of new noble-metal chemistry. Our experimental results provide a rigorous test of first-principles theoretical approaches. Once compounds with useful properties have been shown to exist under ambient conditions, large-scale synthesis techniques (e.g., nitrogen ion irradiation) can be considered. This project will provide Lawrence Livermore with wide-ranging achievements in chemistry under extreme conditions, such as an enhanced ability to perform Raman measurements on relevant materials in the Chapman-Jouguet state, and to identify reaction products.
\end{abstract}

\section{Mission Relevance}

By offering a practical and accurate means for determining the phase boundaries of metals at ultrahigh pressures and temperatures and providing reliable experimental data to core computational efforts, this research is applicable to stockpile-relevant materials such as actinides and alloys. It also supports LLNL's commitment to breakthroughs in fundamental science.

\section{FY06 Accomplishments and Results}

In FY06, we (1) successfully melted iron at approximately $25 \mathrm{GPa},(2)$ used synchrotron nuclear forward scattering to determine the melting point, (3) heated iron to temperatures exceeding $2000 \mathrm{~K}$ using combined internal and laser heating, (4) performed x-ray diffraction on the results, and (5) developed a new optical high-temperature pressure 
scale. During the year we changed our focus to noble-metal chemistry, and major achievements in this area included the synthesis of the first bulk nitride of iridium, and elucidating the structure, stoichiometry, and Raman spectrum of platinum nitride, which was reported in the journal Science.

\title{
Proposed Work for FY07
}

In FY07, the original scope for determining the iron melting curve will be reduced. None the less, we will continue to use combined internal resistive and laser heating to heat iron samples and determine the melting point to a pressure of approximately $50 \mathrm{GPa}$. Cubic boron nitride will be used as the pressure sensor to determine pressure at simultaneous high temperature. The synthesis of new metal nitrides will continue once the structures of iridium and palladium nitride have been conclusively established. Metals to be examined may include rhodium, cobalt, nickel, osmium, ruthenium, iron, silver, and gold.

\section{Publications}

Crowhurst, J. C., et al., 2006. "Synthesis and characterization of the nitrides of platinum and iridium." Science 311, 1277. UCRL-JRNL-216422.

Goncharov, A. F., et al., 2006. "Raman spectroscopy of cubic boron nitride under extreme conditions." Phys. Rev. B. 72, 100104. UCRL-JRNL-218266.

\section{Mitigation of Optical Damage Sites on Ultraviolet Optics}

\section{Christian Mailhiot 05-ERD-066}

\begin{abstract}
The objective of this project is to explore the concept that damage on the surface of an optic can be mitigated to extend the lifetime of the optic in a high-fluence laser system. We will explore mitigation processes utilizing mechanical and chemical techniques for material removal, crack healing, and surface smoothing and characterize the resulting divots in terms of their acceptability with respect to downstream beam intensification. Advanced materials characterization techniques will be used to provide a fundamental understanding of the modifications in materials properties due to the mitigation process. The proposed mitigation efforts will focus on fused silica and potassium dihydrogen phosphate (KDP) frequency-conversion crystals and will emphasize understanding the fundamental materials science of the mitigation processes.
\end{abstract}

One goal is to develop robust, laser-irradiation-based mitigation protocols for fused silica capable of treating damage sites varying in size from $0.02 \mathrm{~mm}$ to $>1 \mathrm{~mm}$ in diameter. Several options will be explored, including (1) varying the optical penetration depth by appropriate choice of the laser wavelength and (2) varying the mitigation laser output characteristics (pulse width, intensity, and spatial beam control). For KDP crystals, we 
propose to examine methodologies based on micromachining and scanned probe-based etching to remove material in the damage region. If we are successful, application of mitigation techniques could significantly extend the useful lifetime of an optic in a highfluence laser system and drastically reduce the associated operational costs.

\section{Mission Relevance}

Development of mitigation techniques that retard damage growth in optical components would significantly benefit efforts to field powerful, fusion-class laser systems by allowing these systems to operate efficiently, reliably, and cost effectively at or above their design specifications. Large, fusion-class laser systems are essential tools for studying weapons physics for stockpile stewardship and inertial-confinement fusion, in support of LLNL's national and energy security missions.

\section{FY06 Accomplishments and Results}

We (1) performed systematic simulations of downstream intensification of laser light as a function of mitigated divot sizes and shapes; (2) established successful mitigation protocols using frequency-doubled and galvo-steered carbon-dioxide lasers on fused silica, enabling the formation of damage-resistant sites up to $0.5 \mathrm{~mm}$ in diameter; (3) designed and built a research-class micromachining tool; (4) developed a successful approach to damage mitigation on KDP crystals using our micromachining technique; (5) established the time, temperature, and humidity dependence of scanned-probe etching on KDP; and (6) used laser-based confocal microscopy to characterize the spectral and temporal response of photoluminescence on damaged and mitigated sites on fused silica, thereby developing a fundamental understanding of the modifications of materials properties due to mitigation.

\section{Proposed Work for FY07}

We will (1) optimize fused-silica mitigation protocols that use frequency-doubled and galvo-steered carbon-dioxide lasers to mitigate large damage sites and, for KPD crystals, optimize micromachining mitigation to create damage-resistant sites; (2) test the efficacy of scanned-probe mitigation techniques on KDP and produce a mechanistic model for surface repair with this method; (3) investigate nondestructive diagnostics-including the correlation of scattered light with observed damage and various excitation and emission wavelengths-for selectively identifying sites with damage propensity; and (4) use microspectroscopy to analyze the photoluminescence of defects in a damage site before and after mitigation and correlate the spectrum with fundamental materials properties.

\section{Publications}

Geraghty, P., et al., 2006. Surface damage growth mitigation on KDP/DKDP optics using single-crystal diamond micro-machining ball end mill contouring. 38th Ann. Symp. Optical Materials for High-Power Lasers, Boulder, CO, Sept. 25-27, 2006. UCRL-CONF-225880. 
Guss, G., et al., 2006. Mitigation of growth of laser initiated surface damage in fused silica using a 4.6- $\mu \mathrm{m}$ wavelength laser. 38th Ann. Symp. Optical Materials for High-Power Lasers, Boulder, CO, Sept. 25-27, 2006. UCRL-CONF-226319.

\title{
A Fracture Mechanics and Tribology Approach to Understanding Subsurface Damage of Fused Silica During Grinding and Polishing
}

\author{
Tayyab I. Suratwala 05-ERD-067
}

\begin{abstract}
The objective of this study is to understand the creation process and characteristics of surface fractures formed during grinding and polishing of brittle materials such as glass. We will experimentally characterize various surface cracks as a function of a number of typical finishing steps. In addition, the effect of key process parameters on the surface damage of fused silica will be measured to develop a global working model to predict surface damage. This project will greatly advance the scientific knowledge of microscopic mechanical damage from grinding and polishing, and will enable optimization of surface finishing processes.
\end{abstract}

The work will produce a body of materials research and enhance manufacturing fabrication processes, which will benefit efforts to field high-energy, high-power laser systems. It will allow them to operate efficiently, reliably, cost effectively, and at or above their design specifications.

\section{Mission Relevance}

The major benefit of this work is a more science-based approach to the fabrication of optical components, a critical and enabling technology for high-energy, high-power, fusion-class laser systems. These laser systems are essential to the Laboratory's mission in stockpile stewardship because of their central role in understanding weapons physics and materials under extreme conditions of temperature, pressure, and strain rate. More generally, this effort will help advance Lawrence Livermore's efforts in optical materials science and laser technology.

\section{FY06 Accomplishments and Results}

In FY06, we developed two models to understand the subsurface damage (SSD) results we obtained in FY05: an elastic fracture-mechanics grinding model to describe the SSD observed, and a finite-difference, three-dimensional (3D) model to simulate the surface topology of a surface with fractures as a function of etch time. We also performed experiments focusing on finishing process variables and how they affect SSD, and made progress in the 3D mapping of static indentations on fused silica. The results of this work provides useful rules-of-thumbs in designing and analyzing optical fabrications processes to make SSD-free optical surfaces. To date, this project has resulted in several conference presentations and proceedings and a peer-reviewed journal article. 


\title{
Proposed Work for FY07
}

In FY07, one major focus will be on characterizing and understanding the effects of "rogue" particles present during polishing, including their effect on SSD and scratch characteristics. The results of these experiments will be complimented by an offline experimental setup to make individual controlled scratches as a function of load, velocity, indentor shape and stiffness, and lubrication. Experiments and modeling also will continue on SSD etching. We will use measured crack and scratch distributions and correlate measured etching behavior with that predicted by our recently developed etching model. Finally, we will continue our effort to understand the plastic deformation and densification of fused silica during static indentation.

\section{Publications}

Menapace, J., et al., 2005. MRF applications: measurement of process-dependent subsurface damage in optical materials using the MRF wedge technique. SPIE Boulder Damage Symp. XXXVII, Boulder, CO, Sept. 19-21, 2005. UCRL-PROC-217271.

Menapace, J., et al., 2005. Utilization of magnetorheological finishing as a diagnostic tool for investigating the three-dimensional structure of fractures in fused silica. SPIE Boulder Damage Symp. XXXVII, Boulder, CO, Sept. 19-21, 2005. UCRL-ABS-212754.

Miller, P., et al., 2005. The distribution of subsurface damage in fused silica. SPIE Boulder Damage Symp. XXXVII, Boulder, CO, Sept. 19-21, 2005. UCRL-ABS-212718.

Suratwala, T., et al., 2006. "Subsurface mechanical damage distributions during grinding of fused silica." J. Non-Crystalline Solids 352(52-54), 5601. UCRL-JRNL-217445.

\section{Characterization of the Effect of Short-Pulse Laser Exposure on Damage Size, Morphology, and Conditioning in Wide-Bandgap Materials}

\author{
Christopher W. Carr 05-ERD-071
}

\begin{abstract}
The objective of this project is to develop a fundamental understanding of how short-pulse laser parameters affect conditioning and initiation of damage in potassium dihydrogen phosphate (KDP) and deuterated KDP (DKDP) crystals and surface damage in fused silica $\left(\mathrm{SiO}_{2}\right)$. We will develop a 100- to 1000-ps short-pulse laser to define optimum protocols for conditioning crystals and initiation of damage precursors in $\mathrm{SiO}_{2}$. The magnitude of the conditioning effect, pinpoint damage density, pinpoint size, and pinpoint morphology will be measured. From our experimental results, we will develop empirical models to provide insight into the underlying physics of energy deposition and material response during exposure to short-pulse lasers.
\end{abstract}


After identifying and optimizing the laser parameters responsible for the conditioning effect, we will use this information to develop offline, laser-based conditioning protocols for crystalline KDP and DKDP and fused $\mathrm{SiO}_{2}$. The conditioning protocols will significantly increase damage resistance of these wide-bandgap materials, enhance their performance, and extend their useful lifetimes in high-energy, high-power, fusion-class laser systems. We also will develop a predictive model that can describe damage in KDP crystals in a large-aperture laser system. If successful, this work will significantly reduce the operating costs of fusion-class laser systems.

\section{Mission Relevance}

Crystalline KDP and DKDP are used for frequency conversion in large, fusion-class lasers. Protocols that extend the useful lifetime of these critical components will have a significant impact on the operating costs of such systems. This work therefore benefits stockpile stewardship and inertial confinement fusion, in support of LLNL's missions in national and energy security.

\section{FY06 Accomplishments and Results}

In FY06, we (1) estimated the starting point and step size for third-harmonic generation (THG) and second-harmonic generation (SHG) crystal conditioning; (2) integrated a regenerative amplifier and power amplifier into the prototype conditioning laser; (3) incorporated THG into the prototype conditioning laser, and tied laser firing to the motion of the translation stage; (4) determined optimum conditioning parameters for THG laser light; (5) developed a semi-empirical model for predicting crystal damage; (6) studied the effect of residual $1 \omega$ light on damage initiation and growth; (7) measured the damage morphology of sites initiated at a variety of pulse durations, and determined damage site growth probability as a function of pulse duration; and (8) measured dependence on pulse duration of the fluence needed to create crystal damage.

\section{Proposed Work for FY07}

In FY07, we will (1) use the sub-nanosecond laser completed in FY06 to determine the optimal conditioning parameters for SHG crystals and determine whether $\mathrm{SiO}_{2}$ can be conditioned, (2) measure the morphology of damage sites produced with various pulse durations between 1 and 10 ns and attempt to determine the optical properties of the sites, (3) measure the effect of wavelength and pulse duration on damage site growth, and (4) develop a model describing laser conditioning in $\mathrm{KDP}$ and $\mathrm{SiO}_{2}$. We expect additional publications from this work.

\section{Publications}

Adams, J. J., et al., 2005. "Wavelength and pulselength dependence of laser conditioning and bulk damage in doubler-cut $\mathrm{KH}_{2} \mathrm{PO}_{4}$." Proc. SPIE 5991, 59911R. UCRL-PROC-216743.

Carr, C. W., and J. M. Auerbach, 2006. "The effect of multiple wavelengths on laserinduced damage in DKDP crystals." Optic. Lett. 31, 595. UCRL-JRNL-214793. 
Carr, C. W., et al., in press. "Complex morphology of laser-induced bulk damage in DKDP crystals." Appl. Phys. Lett. UCRL-JRNL-211938.

Carr, C. W., et al., 2005. "A summary of recent damage-initiation experiments on KDP crystals." Proc. SPIE 5991, 59911Q. UCRL-PROC-216929.

Carr, C. W., et al., 2006. "Techniques for qualitative and quantitative measurement of aspects of laser-induced damage important for laser beam propagation." Meas. Sci. Tech. 17, 1958. UCRL-JRNL-211068.

Feit, M. D, A. M. Rubenchik, and J. B. Trenholme, 2005. "Simple model of laser damage initiation and conditioning in frequency conversion crystals." Proc. SPIE 5991, 59910W. UCRL-PROC-216930.

Norton, M. A., et al., 2005. "Growth of laser damage in $\mathrm{SiO}_{2}$ under multiple wavelength irradiation." Proc. SPIE 5991, 599108. UCRL-PROC-216742.

Trenholme, J. B., M. D. Feit, and A. M. Rubenchik, 2005. "Size-selection initiation model extended to include shape and random factors." Proc. SPIE 5991, 59910X.

UCRL-PROC-216931.

\title{
Molecular Transport in One-Dimensional Lipid Bilayers: A Biological "Smoke Alarm"
}

Aleksandr Noy $\quad$ 05-LW-040

\begin{abstract}
The purpose of this research is to build and characterize one-dimensional (1D) bilayers, new biomimetic nanostructures that consists of a lipid bilayer wrapped around a single carbon nanotube transistor. The lipid bilayer insulates the nanotube from the solution and serves as a matrix for membrane proteins. Use of biomimetic components in a nanoelectronic device allows direct conversion of ion transport events into an electrical signal. We will synthesize 1D lipid bilayers, use these structures to study ion transport through a single protein pore, and in the final year construct a prototype biosensor for specific detection of the botulism toxin.
\end{abstract}

This project will not only create a new class of functional biocompatible nanostructures and use them to perform fundamental studies of molecular transport in these systems, but also will develop a prototype device for specific toxin detection. In addition, this project addresses important scientific questions such as synthesis of 1D organic nanostructures and studies of mass transport in 1D environments, and also establishes an important capability in developing nanowire-based specific biological sensors for homeland security tasks. These studies have the potential to enable several important biophysical applications, such as creation of subcellular-size, ion-selective biocompatible electrodes, or analyzing mass transport through individual protein pores. 


\section{Mission Relevance}

This project has the full potential to bring a set of novel and important capabilities in biodetection and scientific capabilities in biophysical research: the synthesis and characterization of organic nanostructures. This project supports the Laboratory's mission in homeland security and bioterrorism prevention. Biophysical aspects of this research are relevant to DOE's Genomics: GtL initiative. In addition, the project contributes to LLNL's mission in breakthroughs in fundamental science.

\section{FY06 Accomplishments and Results}

In FY06, we (1) completed our device platform; (2) used it to demonstrate the firstever operation of a nanotube transistor suspended in a liquid; (3) characterized the electrical properties of 1D bilayers and their individual components; (4) performed the initial protein-pore sensing experiments; (5) completed studies of polymer self-assembly on nanotube templates, which established the general principles governing such selfassembly; and (6) published the results of our work in high-profile journals.

\section{Proposed Work for FY07}

We will (1) use our bioelectronic device to detect chip-amplified transport through a protein pore and study details of the transport events; (2) demonstrate the simultaneous operation of several carbon nanotube transistors on the chip; and (3) build a prototype sensor for specific differential detection of the botulism toxin, using differential detection with a pair of devices, only one of which will incorporate specific ganglioside receptors for the toxin into the 1D bilayer. The differential signal between the two devices will provide detection specificity.

\section{Publications}

Artyukhin, A. B., et al., 2006. "Controlled electrostatic gating of carbon nanotube FET devices." Nano Lett. 6, 2080. UCRL-JRNL-222278.

Huang, S.-C., et al., 2006. "Persistence length control of the polyelectrolyte layer-by-layer self-assembly on carbon nanotubes." J. Am. Chem. Soc. 127, 14176. UCRL-JRNL-214130. 


\title{
Target Fabrication Science and Technology: An Enabling Strategic Initiative
}

\author{
Peter A. Amendt \\ 05-SI-005
}

\begin{abstract}
This project will establish a new, major capability in target fabrication for fusion ignition, with applications in homeland security and nanotechnology. To this end, we will develop the science and engineering for (1) nanocrystalline grains, (2) graded-dopant materials, (3) high-strength diamond shells, (4) nanoporous metals, (5) high-strength aerogels, (6) atomic-layer deposition on aerogels, (7) interference and advanced lithography, and (8) joining techniques for precision microassembly. We will develop a double-shell ignition prototype target as a test bed for integrating these materials and methods.

This project will advance nanoscience techniques for fabricating targets and other structures by developing (1) nanostructured alloys with extraordinary strength, ductility, and thermal stability; (2) the capability to independently control the surface properties and pore structure of nanocellular materials and relate these to nanomechanical behavior; and (3) advanced lithographic approaches to three-dimensional (3D) nanofabrication on large areas and complex surfaces with nanoscale-relief structures. Insight into all of these issues will lead to a new class of materials with novel properties.
\end{abstract}

\section{Mission Relevance}

This work supports Lawrence Livermore's missions in national and energy security by developing target-fabrication technology for the increasingly complex targets needed in stockpile stewardship and fusion ignition.

\section{FY06 Accomplishments and Results}

In FY06, we (1) measured the tensile properties, strain-rate sensitivity, and activation volume of the target materials; (2) produced a bimetallic, graded inner shell of gold-copper; (3) produced nanoporous copper with a density of $85 \mathrm{mg} / \mathrm{cm}^{3}$; (4) demonstrated 3D nanostructured, gradient-density materials by conformal phase-mask lithography; (5) completed an initial study of a diamond ablator design; (6) completed a feasibility study on the use of glass mandrels for nanocrystalline gold-copper spherical shells; and

(7) completed an initial study to determine the maximum allowable metallic foam pore sizes.

\section{Proposed Work for FY07}

In FY07, we will deliver all the technologies to produce an ignition-ready, double-shell prototype target integrating the world-class material science advances achieved in the previous two years of this project. The goals of the prototype are to demonstrate

(1) concentricity of the inner and outer shells to within $2 \mu \mathrm{m}$, (2) root-mean-square surface 
smoothness of less than $50 \mathrm{~nm}$ on the outer surface of the bimetallic nanocrystalline inner shell, (3) equivalent inner-shell strength to contain $800 \mathrm{~atm}$ of hydrogen fuel at room temperature, and (4) nanoporous metallic foams $\left(<100 \mathrm{mg} / \mathrm{cm}^{3}\right)$ with submicrometer pore size that are formed into a spherical shell.

\title{
Publications
}

Amendt, P., 2006. "Bell-Plesset effects for an accelerating interface with contiguous density gradients." Phys. Plasma 13, 042702. UCRL-JRNL-217862.

Biener, J., A. M. Hodge, and A. V. Hamza, 2006. "Microscopic failure behavior of nanoporous gold." Appl. Phys. Lett. 87, 121908. UCRL-JRNL-209008.

Biener J., et al., 2006. "Diamond ablators for inertial confinement fusion." Fusion Sci. Tech. 49, 737. UCRL-JRNL-213214.

Biener, J., et al., in press. "Nanoporous metal: combining strength and porosity." Nanoletters. UCRL-JRNL-221479.

Jankowski, A. F., et al., 2006. "Nanocrystalline growth and grain-size effects in $\mathrm{Au}-\mathrm{Cu}$ electrodeposits." Thin Solid Films 494, 268. UCRL-JRNL-210132.

Kucheyev, S. O., et al., 2006. "Monolithic, high surface area, three-dimensional $\mathrm{GeO}_{2}$ nanostructures." Appl. Phys. Lett. 88, 103117. UCRL-JRNL-214093.

Kucheyev, S. O., et al., 2006. "Nanoengineering mechanically robust aerogels via control of foam morphology." Appl. Phys. Lett. 89, 041911. UCRL-JRNL-218677.

Kucheyev, S. O., et al., 2006. "Surface-enhanced Raman scattering on nanoporous Au." Appl. Phys. Lett. 89, 053102. UCRL-JRNL-220317.

Zepeda-Ruiz, L. A., et al., 2006. "Atomistic simulations of grain boundary pinning in $\mathrm{Cu}-\mathrm{Fe}$ alloys." Appl. Phys. Lett. 87, 231904. UCRL-JRNL-212649.

Zielasek, V., et al., in press. "A new gold catalyst: nanoporous gold foams." Angew. Chem. Int. Ed. UCRL-JRNL-222533.

\section{Transformational Materials Initiative}

\author{
Randall L. Simpson 06-SI-005
}

\section{Abstract}

The goal of this project is to provide the underlying science and technology for converting the U.S. nuclear weapons complex to one that is smaller, safer, and more agile. We will create new materials and technologies to facilitate the nuclear weapons complex transformation by (1) reducing the cost and time required to produce and maintain the 
stockpile, (2) enhancing weapon safety, (3) ensuring future stockpile longevity, and (4) optimizing stockpile performance. Our multidisciplinary team combines capabilities in materials synthesis, characterization, theory, and modeling to deliver cutting-edge advances in high explosives, sensing, metals, and multifunctional materials.

The project will create basic scientific and technical capabilities that will help transform the nuclear weapons complex. Success will make it more efficient and effective by achieving fundamental scientific advances in synthesis chemistry, metallurgy, dynamic experiments, and molecular design.

\section{Mission Relevance}

This project will achieve enhanced reliability, improved safety, easier manufacturing, and reduced surveillance requirements for the nuclear weapons complex, and help ensure the continued success of the Stockpile Stewardship Program.

\section{FY06 Accomplishments and Results}

After a midyear start in April 2006, we (1) created a new class of solvents that allows for processing high explosives with optimal shock insensitivity, (2) developed novel polymers that were shown to improve the strength of processed explosives, (3) narrowed eight candidate sensing technologies to three focus areas in scientific collaboration with five universities and two other weapons labs, (4) produced two new composite alloys and a new explosive method for material property tests, and (5) synthesized a novel nanoscopic material for the catalytic scavenging of hydrogen. All efforts were extensively supported by advanced computer simulations, including molecular dynamics, continuum dynamics, and density functional methods.

\section{Proposed Work for FY07}

In FY07, we will (1) study and model crystal growth of explosive molecules from the new solvents using spectroscopy and atomic force microscopy; (2) develop a prototype fiberoptic gas sensor with response to a broad range of gases, and study acoustic sensors and devices that are remotely powered by radiofrequency energy; (3) optimize the new alloyprocessing methods, and perform analysis using the new explosive testing method; and (4) synthesize and test new nanoscale catalytic compounds, with further density functional and molecular dynamics calculations being performed to guide the effort.

\section{Publications}

Pocha, M. D., et al., in press. "Miniature accelerometer and multichannel signal processor for fiberoptic Fabry-Perot sensing." IEEE Sensor J. UCRL-JRNL-2222923-DRAFT. 


\title{
Critical Materials Issues for Generation IV Reactors
}

\author{
Magdalena A. Serrano De Caro 06-ERD-005
}

\begin{abstract}
In this project, we propose to develop predictive tools to calculate structural and mechanical properties of iron-chromium alloys, which form the base matrix of advanced ferritic/martensitic steels required for fuel cladding and structural components in Generation IV lead-cooled fast reactors. Using massively parallel computing, two novel modeling approaches at atomistic and mesoscale levels will be merged to describe the mechanical behavior of these alloys. For validation purposes, our physically based multiscale study will be compared with existing experimental data over a range of length and time scales.
\end{abstract}

We expect to obtain (1) a detailed physical understanding of iron-chromium alloy behavior and prediction of mechanical properties (swelling, radiation hardening, embrittlement, creep rupture, and fatigue susceptibility) that are critical for resolving technological issues associated with high temperatures and fluence in future burner reactor designs; (2) a sound connection between atomistic-mesoscales with polycrystal plasticity that bring us a step closer to "real experiment" simulation and to engineering-scale materials strength models for irradiated alloys; and (3) the first building block of an integrated simulation platform for modeling radiation damage in nuclear materials. Advanced simulations of this kind will help identify candidate structural materials for future reactor systems.

\section{Mission Relevance}

This project supports Laboratory and DOE missions in long-term energy security by developing scalable materials-science codes to predict nuclear materials properties and their performance under extreme conditions and assisting efforts to develop future reactor concepts such as the Advanced Burner Reactor envisioned by the DOE's Global Nuclear Energy Partnership initiative. In addition, materials properties codes developed in this project can be used to predict radiation damage to materials important to LLNL's stockpile stewardship mission.

\section{FY06 Accomplishments and Results}

In FY06, we (1) achieved our first task of developing and validating our thermodynamicsbased and multicomponent, semi-empirical iron-chromium interatomic potential intended for future modeling of the evolution of precipitation microstructures under irradiation; (2) began combined Monte Carlo-molecular dynamics simulations of heterogeneous segregation in iron-chromium alloys and the first studies of phase-separation thermodynamics; (3) successfully completed enhancements needed in dislocation dynamics methodology to treat hardening scenarios by generating mobility functions for the mesoscale stage in face-centered-cubic (fcc) and body-centered-cubic (bcc) alloys; and (4) implemented defect-dislocation interactions in dislocation dynamics for fcc alloys 
and completed preliminary steps for bcc alloys. These models include elements to extend simulation capabilities to other alloying materials.

\section{Proposed Work for FY07}

In FY07, we will link both mesoscale and atomistic scales by providing advanced computational tools for predicting microstructure evolution (i.e., number densities of irradiation-produced defect clusters). Specifically, we will (1) perform molecular dynamics studies of point-defect cluster transport and phase separation thermodynamics in concentrated iron-chromium alloys, (2) continue preliminary atomistic studies of dislocation hardening to obtain mobility functions for iron-chromium alloys, (3) extend work on defect-dislocation interactions in dislocation dynamics codes to the case of iron-chromium alloys, and (4) initiate a parameter-free, crystal-plasticity approach implementing a hybrid quasicontinuum-dislocation dynamics methodology. The models also will include elements to extend simulation capabilities to other alloying materials.

\section{Publications}

Caro, A., et al., 2006. "Implications of ab initio energetics on the thermodynamics of $\mathrm{Fe}-\mathrm{Cr}$ alloys." Appl. Phys. Lett. 89, 121902. UCRL-JRNL-225773.

Caro, M., et al., 2006. Critical issues on materials for Generation-IV reactors. American Nuclear Society, Ann. Mtg., Reno, NV, June 4-8, 2006. UCRL-POST-221431.

Marian, J., and A. Caro, 2006. "Moving dislocations in disordered alloys: connecting continuum and discrete models with atomistic simulations." Phys. Rev. B 74, 024113. UCRL-JRNL-330895.

Marian, J., and J. Knap, 2006. A quasicontinuum study of scale effects in uniaxially compressed Au nanopillars. 43rd Ann. Technical Mtg. Society of Engineering Science, University Park, PA, Aug. 13-16, 2006. UCRL-PRES-223692.

Martinez, E., et al., in press. "Atomistically-informed dislocation dynamics in fcc crystals." J. Mech. Phys. Solid. UCRL-JRNL-224304.

\section{Time-Resolved Phase Transitions via Dynamic Transmission Electron Microscopy}

Bryan W. Reed

$$
\text { 06-ERD-007 }
$$

\section{Abstract}

This project will use the unique capabilities of the dynamic transmission electron microscope (DTEM) to capture the kinetics of phase transformations and transient 
phenomena at high temperature and pressure with a temporal resolution never before possible. This will include characterization of the propagating reaction front in reactive multilayer foils and of conformational changes in tetranitro tetrazacyclo-octane (HMX) high-explosive molecules residing in a crystalline lattice. The measurements will be complemented by computer simulations for experimental design and interpretation, while the experimental results will serve to validate and constrain the models by accessing the very same nanometer and nanoscond scales as the simulations.

The DTEM has a unique ability to probe material processes at high spatiotemporal resolution, allowing direct measurement on scales that are usually only accessible via computer simulation. Our research subjects are interesting not only in a practical sense, but also in terms of new fundamental science that comes from coupling multiple processes such as thermal and atomic diffusion, chemical reactions, and molecular conformation changes on multiple-length scales. With the DTEM, these processes become directly measurable, enabling us to experimentally explore the details of transformations and thereby test, constrain, and extend the models. Visible success in this area will help advance the next generation of DTEM-like instruments.

\section{Mission Relevance}

The high-speed, high-resolution diagnostics of material behavior under extreme conditions developed in this project will directly support Lawrence Livermore's national security mission in stockpile stewardship.

\section{FY06 Accomplishments and Results}

In FY06, we conducted DTEM, conventional in situ TEM, and ex situ optical experiments in concert with computer simulations to develop a complete understanding of the transformations. Results from reactive multilayer foils suggest a surprising time lag between arrival of the thermal front and generation of new material phases. We are using computer modeling to better understand this. The HMX crystals had a high tendency to sublimate rather than transform in vacuum-we demonstrated that the transformation is nearly impossible to observe with conventional in situ TEM, but can be imaged with the DTEM. Computational models have helped us to interpret this behavior in terms of the underlying thermodynamics, particularly the importance of free volume for nucleation. Publications describing the results are in preparation.

\section{Publications}

Armstrong, M. R., et al., 2006. Kinetics of the beta-delta phase transition in nitramine octahydro-1,3,5,7-tetranitro-1,3,5,7-tetrazocine studied using dynamic transmission electron microscopy. 16th Intl. Microscopy Conf., Sapporo, Japan, Sept. 3-8, 2006. UCRL-ABS-222702.

Kim, J. S., et al., 2006. Study of metastable phase formation in Al/NiV reactive multilayer foils using dynamic transmission electron microscopy (DTEM). 16th Intl. Microscopy Conf., Sapporo, Japan, Sept. 3-8, 2006. UCRL-ABS-218534. 


\title{
Fundamental Investigation of Laser-Induced Surface Damage in Optical Materials
}

\author{
Jeffrey D. Bude 06-ERD-035
}

\begin{abstract}
The objective of this research is to develop a fundamental understanding of laser-induced surface damage in optical materials-specifically, in fused silica. Despite its excellent ultraviolet transparency, silica undergoes damage under high ultraviolet fluence. Although intrinsic bulk damage is relatively well understood, extrinsic surface damage dominant in high-quality silica is not. This work will develop a suite of high-resolution optical techniques and advanced computational models to help clarify the processes behind surface damage in fused silica.
\end{abstract}

We expect to (1) identify the defects that absorb sub-bandgap light and lead to surface damage, (2) develop methods to directly measure laser-energy absorption in the nearsurface region of fused silica for fluences below the damage threshold, and (3) correlate energy absorption to damage probability. These tasks will help address major gaps in our understanding of the basic processes that lead to extrinsic surface damage: free-carrier generation by defects, free-carrier absorption in silica, and thermally induced runaway absorption.

\section{Mission Relevance}

This work directly addresses stockpile stewardship and laser fusion challenges by optimizing use of large fusion-class laser systems. It will also serve to establish science-based rules for optics reliability predictions, improve damage diagnostics, and suggest pathways to improve damage resistance in optical materials. By furthering the understanding of structural and electronic transitions in glasses and disordered materials-a frontier problem in condensed-matter physics-this project contributes to the Laboratory's mission in breakthroughs in fundamental science and technology.

\section{FY06 Accomplishments and Results}

Using experimental techniques and computational modeling, in FY06 we (1) assembled a platform for defect photoluminescence (PL) imaging; (2) generated fully three-dimensional (3D) PL images of defects in damage sites; (3) began correlating PL images to damage susceptibility; (4) developed the capability for concurrent, nonlinear PL and Raman temperature measurements for direct energy absorption in damage sites; (5) performed initial electronic calculations of silica structure; (6) identified possible absorption pathways; (7) conducted optical absorption calculations for amorphous silica structures showing qualitatively that excited-state absorption can generate free carriers through localized defects; and (8) began creating a 2D transport model based on our experiments and simulations. 


\title{
Proposed Work for FY07
}

In FY07, we will (1) complete the setup and calibration of the PL imaging system; (2) image a variety of damage and mechanically engineered sites; (3) identify defect types, lifetimes, and emission properties; (4) correlate defect signatures with damage; (5) complete Raman temperature measurements and perform two-color pump-probe, free-carrier absorption measurements on damage sites; (6) add hybrid functional and oneparticle Greens function with screened Coulomb interaction techniques to improve energy calculations; (7) quantify absorption and explore the effects of disorder on defect cluster absorption and free-carrier absorption; and (8) perform measurements of temperaturedependent damage thresholds.

\section{Publications}

Carr, C. W., et al., 2006. A comparison of laser-induced damage initiation in KDP and $\mathrm{SiO}_{2}$. 38th Symp. Optical Materials for High Power Lasers, Boulder, CO, Sept. 25, 2006. UCRL-PRES-224739.

\section{Large-Aperture Optics Performance}

\author{
Thomas G. Parham 06-ERD-054
}

\begin{abstract}
The performance of large-aperture optics has traditionally limited the maximum output of large fusion-class lasers. Prior work has not employed realistic testbeds that allow largeaperture optics to be studied in the same way they will actually be used in large laser systems. Especially important is the effect of unconverted $1 \omega$ and $2 \omega$ light on $3 \omega$ damage. This project will evaluate large optics at full aperture, with unconverted light present and with the statistical fluctuations that are typical of large-aperture lasers. Specialized optomechanical hardware that edge-illuminates optics will be coupled with extensive laser diagnostics in an existing large-aperture laser system called the precision diagnostic system (PDS) to allow in situ analysis of damage initiation and growth.

Materials research that enhances our understanding of complex mechanisms involving the response of optical materials to intense laser irradiation will benefit the development of ever more powerful, fusion-class laser systems and allow such lasers to operate efficiently and reliably at or above their design specifications. Our modeling efforts will allow operators of fusion-class laser systems to predict effects of experiments on the lifetime of optics, and allow them to optimize experiments to minimize the costs of replacing optical components. In addition, this project will help ensure LLNL's continued leadership in the fields of optical materials science and laser technology.
\end{abstract}




\title{
Mission Relevance
}

This work will add substantially to the knowledge base of optical materials under the intense laser illumination of fusion-class lasers systems, and allow us to validate our theoretical and stochastic models. This work supports the Laboratory's mission in the national-security area of stockpile stewardship, because safety and reliability of the nuclear stockpile in the absence of testing will rely heavily on experimental data from fusion-class laser systems to validate complex computer simulations.

\section{FY06 Accomplishments and Results}

We conducted a series of experiments on the PDS that exposed optics to a range of wavelengths, fluences, and pulse lengths and shapes while using edge-illuminated, full-aperture image analysis to track damage initiation and growth. We used a new diagnostic with $<10-\mu \mathrm{m}$ detection capability that enabled in situ damage assessment. In these experiments, we observed that (1) intensification due to upstream flaws was a predominant contributor to damage initiation, (2) initiation of damage is strongly influenced by residual unconverted light, (3) filamentation occurs at lower intensities than expected, and (4) existing physics models failed to predict the observed damage initiation, prompting intensive revision of the models.

\section{Proposed Work for FY07}

In FY07 we plan to use the results from FY06 to conduct a second series of shots on PDS using different optics and a different optical configuration designed to minimize the impact of upstream flaws. Flaw specifications for these optics will be defined using propagation models updated based on the FY06 data. The FY07 campaign will test the efficacy of both the flaw specs and the new optical configuration over a range of fluences and pulse shapes. After these experiments, we will update our models with damage rules appropriate for large-aperture, high-energy, high-power laser systems and begin publishing the results in peer-reviewed journals.

\section{Understanding Shape Control in Nanoparticle Synthesis}

Christine A. Orme 06-LW-090

\begin{abstract}
Enormous technological advantages could be gained by understanding how to control the deposition, growth, and molecular-binding events between metal and organic molecules. One arena where this is particularly true is in the use of organic molecules to modify the shape of nanoparticles for sensing applications. In this proposal, we focus on the fundamental interactions at surfaces that drive these shape changes. We will combine
\end{abstract}


nanoparticle synthesis with several in situ techniques-such as atomic force microscopy and micro-ellipsometry-to monitor atomic-step motion and molecular binding events at surfaces during crystal growth. These experimental results will be used as input to model the shape evolution.

This project will provide a capability to understand metal-organic interactions at surfaces. It enables a more fundamental understanding of the self-assembly process with regards to the very interesting, but predominately empirical, fields of particle synthesis, electrodeposition, and corrosion. We expect to develop mechanistic models of how particular chemical moieties can be used to influence metal and metal oxide growth and dissolution.

\section{Mission Relevance}

By contributing to a better understanding of how to control the deposition, growth, and molecular-binding events between metal and organic molecules, this project contributes to developing a scientific platform for sensing and separation technologies for national security and homeland security missions, to synthesizing materials for targets in support of stockpile stewardship experiments, to understanding the corrosive effects of organic acids produced by microbes in nuclear waste facilities for missions in energy security and environmental management, and to understanding how polymeric materials degrade metallic weapons components for the stockpile stewardship mission.

\section{FY06 Accomplishments and Results}

We successfully imaged the nucleation and growth of silver in the presence and absence of the modifier cetyltrimethylammonium bromide (CTAB), which is known to induce rod-like growth during silver nanoparticle synthesis. The results of these studies-the first-ever in situ electrochemical deposition studies detailing the effects of CTAB concentration and potential at the atomic level-demonstrate that CTAB changes the growth mode and that certain facets stop growing, inducing a shape change. In addition, we used electron backscattered diffraction to correlate the effects of metal grain orientation on silver growth rate and morphology. These results are being used as input to our model. This work also resulted in one invited talk and two conference presentations.

\section{Proposed Work for FY07}

Research in FY07 will focus on (1) in situ imaging of nucleation and atomic-step motion during electrodeposition, (2) measurement of orientation-dependent adsorption using spatially resolved ellipsometry, and (3) modeling shape evolution using the computationally efficient level-set method. Experiments are planned to elucidate the effect on silver growth of chemical moiety (hydroxyl, carboxyl, amine, imidazole, and pyrrole) and to develop shape models for each organic modifier based on kinetic Wulff plots. 


\title{
Publications
}

Gray, J. J., et al., 2006. An in situ AFM study of the influence of organic additives on metal growth. Electrodeposition Gordon Research Conf., New London, NH, July 30-Aug. 8, 2006. UCRL-ABS-221473.

Orme, C. A., et al., 2006. A molecular view of adsorbate effects during crystallization. Electrodeposition Gordon Research Conf., New London, NH, July 30-Aug. 4, 2006.

UCRL-ABS-208834.

\section{Enhanced Efficiency Chip Slapper for Detonator Applications Using a Metal Hydride as a Solid-State Gas Gun}

Paul R. Wilkins

06-FS-015

\begin{abstract}
The objective of this study is to launch, with greater efficiency and safety, weapon-system flyers at velocities of several kilometers per second. Current low-energy detonators for NNSA systems have requirements for minimum operation energy. These systems use a primary explosive, which is affected by electrostatic discharge, friction, and impacts. Our proposed metal-hydride slapper requires lower energy operation and allows use of a secondary explosive, resulting in a safer overall system. The design will incorporate a metal-hydride detonator bridge, which, upon bursting, will transfer energy from the plasma to the stored hydrogen. The internal energy stored in the hydride lattice at high densities is released, causing hydrogen to expand in the same manner as a hydrogen gas gun, pushing a thin layer of Kapton or Parylene deposited on the metal to a high velocity. We wish to achieve threshold energy levels for slapper detonators to reliably initiate with a jitter of $10 \mathrm{~ns}$. Low-energy initiation systems using primary explosives can thus utilize secondary explosives using energy stored in the hydride, in place of the deposited burst energy.
\end{abstract}

The principal expected result is a device with higher slapper flyer velocities that are capable of initiating secondary high explosives at lower charge voltage. This will be a unique energy-storage system, where a metal-hydride plasma burst releases bound hydrogen at high energy densities to drive flyers to higher velocities. Lighter metal hydrides offer the greatest velocity increase and lower threshold burst-current conditions, limiting the energy needed.

\section{Mission Relevance}

This project supports Lawrence Livermore's national security mission by developing technology for safer and more efficient detonators. Other potential applications involve gas generators, pyrotechnic initiators, and small millimeter-scale, one-dimensional (1D) shock generators. 


\section{FY06 Accomplishments and Results}

In FY06, the DOE Kansas City Plant fabricated 12 slappers using an existing mask with both palladium and titanium. These metal slappers were coated with 0.3-mil Kapton flyers, fired with a capacitive discharge unit capable of 8-kA currents, and the velocity of the bridges (with and without the stored hydride) was measured with a photonic Doppler velocimeter. The velocity data was compared with simple 1D hydrocodes-the efficiency of the chip slapper seems to be higher with the hydride material. We obtained photonic Doppler velocimeter data for about 90 to 140 ns into the launch, and were thus able to determine the acceleration but not ultimate velocity. Early results indicate higher acceleration rates for hydride materials. 
Laboratory Directed Research and Development

\section{Mathematics and Computing Sciences}




\title{
Biological and Synthetic Nanostructures Controlled at the Atomistic Level
}

Andrew J. Williamson 03-SI-001

\begin{abstract}
Nanotechnology holds great promise for many application fields, ranging from the semiconductor industry to medical research to national security. Novel, nanostructured materials are the fundamental building blocks upon which all these future nanotechnologies will be based. In this project, we are conducting combined theoretical and experimental investigations of the synthesis, characterization, and design techniques that are required to fabricate semiconducting and metallic nanostructures with enhanced properties. We are focusing on developing capabilities with broad applicability to a wide range of materials and that can be applied both to existing and future nanomaterials.
\end{abstract}

We are building expertise, computer simulation and modeling codes, chemical and vapor deposition synthesis techniques, and surface-sensitive spectroscopic characterization techniques for studying novel, nanostructured materials. In addition, we are identifying nanomaterials for designing biological sensing, detection, and separation devices. Our results have generated numerous high-profile scientific publications that have facilitated the recruitment of new postdoctoral researchers and staff to work at Lawrence Livermore. In addition, we have set up and consolidated new laboratories for materials synthesis and characterization.

\section{Mission Relevance}

This research will help to predict, develop, and design semiconductor materials with tailored properties for advanced biodetection needs in support of the LLNL's national security mission. It will provide a scientific foundation for the creation of next-generation technologies based on manipulating and controlling matter at the nanoscale, in support of the Laboratory's mission in breakthroughs in fundamental science and technology.

\section{FY06 Accomplishments and Results}

In FY06, we (1) completed synthesis, characterization, and modeling of silicon and germanium nanoparticles with controlled size and surfaces; (2) optically characterized nanoparticle superlattices; (3) completed studies examining cadmium selenide in the nuclei of cells; and (4) demonstrated the chromatographic separation capabilities of microfluidic channels. This project developed a range of first-principles simulation capabilities for studying the synthesis and characterization of nanomaterials. These simulations, combined with state-of-the-art synthesis and characterization experiments, have advanced our knowledge of nanoscale semiconductors and how their structural and optical properties can be harnessed for chemical and biological sensing technologies. 


\title{
Publications
}

Dal Negro, L., et al., 2005. "Light emission from silicon-rich nitride nanostructures." Appl. Phys. Lett. 88, 183106. UCRL-JRNL-216732.

Drummond, N., et al. 2006. "Electron emission from diamondoids: a diffusion quantum Monte Carlo study." Phys. Rev. Lett. 95, 096801. UCRL-JRNL-209819.

Kim, Y. H., et al., 2006. "'Non-dissociative adsorption of $\mathrm{H}_{2}$ molecules in light-element doped fullerenes." Phys. Rev. Lett. 96, 016102. UCRL-JRNL-209820.

LeMieux, M. C., et al., in press. "Single functional group interactions with carbon nanotube sidewalls." Nat. Mater. UCRL-JRNL-220263.

\section{ICE: The Image Content Engine}

James Brase 03-SI-003

\begin{abstract}
Advancements in imaging-sensor technologies (particularly for remotely sensed images) are resulting in volumes of data that overwhelm intelligence analysts. The goal of this project is to develop a framework - the Image Content Engine (ICE)-that will allow analysts to interpret massive volumes of imagery in a timely fashion. The ICE approach focuses the attention on relatively few small areas with specified attributes. This will be achieved by capturing content extracted from images using model-based, object-detection methods.
\end{abstract}

The ICE framework will allow content extracted from imagery-the most voluminous data source-to be seamlessly integrated with information from other sources. This will help break down the compartmentalized nature of analyst-based interpretation in the defense and intelligence communities. ICE also will greatly increase the productivity of image analysts by very efficiently focusing attention on potential objects of interest. In addition, the ICE approach applies to other areas of experimental science (e.g., physics, biology, and environmental science), in that mining massive archives of complex measurement data for new patterns and relationships is an emerging model for discovery in modern science.

\section{Mission Relevance}

The work supports Lawrence Livermore's mission in national security by developing new technology to perform efficient searches of large image databases, and to allow the content extracted from images to be seamlessly integrated with information from other sources. This enables analysts to submit sophisticated queries relevant to defense, intelligence, counterterrorism, deployment strategies, determining the functions of enigma facilities, and detecting the production of weapons of mass destruction. 


\title{
FY06 Accomplishments and Results
}

In FY06, we (1) completed development of the parallel version of ICE, (2) characterized the computational performance of this version in large sets of images, and (3) characterized data movement through the computing cluster and optimized performance. The ICE project has succeeded in developing a new approach to modelbased object detection that extends to very large image sets and scales to large search problems. This capability is enabling the Laboratory to perform image analysis applications in nonproliferation and intelligence applications that were not previously possible.

\section{Publications}

Chen, B. Y., and D. W. Paglieroni, 2006. Using gradients, alignment, and proximity to extract curves and connect roads. SPIE Defense and Security Symp., Orlando, FL, Apr. 17-21, 2006. UCRL-CONF-218522.

Grant, C., S. Nikolaev, and D. W. Paglieroni, 2006. Using component weighting with gradient direction matching. SPIE Defense and Security Symp., Orlando, FL, Apr. 17-21, 2006. UCRL-CONF-220030.

Miller, W. M., et al., 2006. Exploiting data parallelism in the Image Content Engine. SPIE Defense and Security Symp., Orlando, FL, Apr. 17-21, 2006. UCRL-CONF-219867.

Paglieroni, D. W., and W. G. Eppler, in press. "Resolution analysis for gradient direction matching of object model edges to overhead images." Int. J. Comput. Vis.

UCRL-JRNL-217038.

\section{Detection and Tracking in Video}

\section{Chandrika Kamath 03-ERD-031}

\begin{abstract}
In this project, we are developing robust, accurate, and near-real-time techniques for detecting and tracking moving objects in video from a stationary camera. These techniques allow us to model interactions among the objects, thereby enabling us to identify normal patterns and detect unusual events. Our algorithms and software include techniques to separate the moving foreground from the background, extract features representing the foreground objects, track these objects from frame to frame, and postprocess the tracks for display. The project focuses on video of objects of different sizes, moving at different speeds, taken under less-than-ideal conditions that include occlusions, changing illumination, poor weather, and low resolution and frame rate.
\end{abstract}

If successful, this project will produce robust and accurate technology for video detection and tracking under less-than-ideal conditions. It will enhance existing algorithms to address these situations, allowing us to better understand their limitations, which in turn, will determine the conditions under which successful surveillance is possible. The 
algorithms and software will be applied to spatiotemporal data from computer simulations and to mine text, audio, image, and video data simultaneously.

\title{
Mission Relevance
}

The capability to detect and track in video supports the national security mission of Lawrence Livermore by enabling new monitoring and surveillance applications for counterterrorism and counterproliferation.

\section{FY06 Accomplishments and Results}

In FY06, we (1) completed an investigation of block matching methods for tracking, to enable more robust and accurate tracking software; (2) authored a technical report summarizing our results; and (3) presented experimental comparison of block matching techniques for detection of moving objects at a conference for the International Society for Optical Engineering. We have shown that it is possible to track multiple objects over the long term in video data. By combining simple algorithms, we obtained robust techniques for detecting moving objects, which performed well on videos taken under a variety of situations. The algorithms and software developed are now being applied to tracking problems in both simulations and experiments.

\section{Publications}

Love, N. S., and C. Kamath, 2006. An empirical study of block matching techniques for detection of moving objects. UCRL-TR-218038.

Love, N. S., and C. Kamath, 2006. "An experimental comparison of block matching techniques for detection of moving objects," Proc. SPIE 6312. UCRL-CONF-221486.

\section{Three-Dimensional Vectorial Time-Domain Computational Photonics}

Jeffrey S. Kallman

04-ERD-004

\begin{abstract}
Three-dimensional (3D) time-domain (TD) design tools are fundamental to developing new technologies to create all-optical nonlinear systems for data generation, transmission, manipulation, and detection. This project has developed state-of-the-art simulation tools for the design and analysis of next-generation 3D photonic integrated circuit devices. The simulation codes developed in this project include models for optical gain and nonlinearities, and microscopic, non-uniform, inhomogeneous structures. This new suite of 3D tools is general enough to be adapted to specialized problems in several areas, and flexible enough to be applicable to the design of future mixed-signal systems and stand-alone systems in separate areas of the electromagnetic spectrum. This project
\end{abstract}


has resulted in a set of 3D TD tools that designers can use to model new, complex photonic devices, and to advance scientific understanding. By developing this unique modeling capability, LLNL will maintain a leadership role in the worldwide scientific and computational community, and be better positioned to carry out our core missions. We expect to publish, patent, and license (where possible) the results of this work.

\title{
Mission Relevance
}

New capabilities developed in this project will benefit LLNL's national security missions that rely heavily on advanced photonics. Applications include encryption circuits for secure communication and remote sensing for counterproliferation and nonproliferation missions; high-bandwidth optics for advanced laser science and high-density optical interconnects for high-performance computing, both in support of stockpile stewardship; and microsensors for weapons miniaturization.

\section{FY06 Accomplishments and Results}

In FY06, we (1) incorporated carrier dynamics, time-varying nonlinear material models, gain, spontaneous emission, and multiscale simulation into the full-wave 3D linear model EMSolve; (2) used the resulting code to perform full-wave 3D simulations of the Auston switch terahertz source and receiver; and (3) extended the approximate scalar 2D nonlinear model Quench2D to a wide-angle 3D beam-propagation method model incorporating polarization effects. Overall, this project has advanced the EMSolve and Quench code suites significantly. Applications for the codes developed include the Laboratory's terahertz effort, next-generation x-ray sensor research, and x-ray beam shaping optics for the Linac Coherent Light Source.

\section{Publications}

Koning, J. M., 2006. "Terahertz photo-conductive antenna array power scaling simulations." Proc. IEEE APS. UCRL-CONF-218057-DRAFT.

\section{Locally Adaptive Mesh Refinement for Linearly Scaling Electronic Structure Calculations}

Jean-Luc Fattebert 04-ERD-012

\begin{abstract}
We propose to investigate a new scalable algorithm for efficient electronic structure calculations, the most computationally expensive part of first-principles molecular dynamics simulations. In current application codes, the algorithm scales as the cube of
\end{abstract}


the number of electrons and thus limits the size of tractable simulations. Our goal is to develop a new algorithm that scales linearly with the number of electrons while preserving the accuracy of standard methods, and investigate a new way of representing the electronic structure using localized orbitals. Fine grids will be used to resolve the "center" of localized wave functions, and coarser grids will represent the tails of the functions. The new approach will use the SAMRAI parallel adaptive mesh refinement framework.

The goal of this research is to develop a fast and efficient algorithm for electronic structure calculations that may be used as the core of a first-principles molecular dynamics code. This algorithm could potentially reduce by more than one order of magnitude the cost of simulations of physical systems of the order of 1,000 atoms, thus allowing larger simulations and longer time scales and opening the possibility of investigating new physical phenomena.

\section{Mission Relevance}

Achieving an efficient, accurate, and scalable algorithm for computing the electronic structure of a system with many atoms would have a large impact in many research fields that have applications to stockpile stewardship, such as molecular dynamics simulation of materials or fluids at high temperature and pressure, and to determine the equation of state of various materials.

\section{FY06 Accomplishments and Results}

The new concept of a "locally active" variable was implemented in the SAMRAI library to enable variable specific local mesh refinement. This concept reduces numerical operations by skipping computation and not allocating data on fine patches when a coarse mesh representation is sufficient. Using these new functionalities, we developed a parallel, finiteelement electronic structure code in which electronic structure is described by a set of localized orbitals, representing their core on a fine mesh and tails on a coarser mesh. This project, although demonstrating the concept of reduced computational cost with system size, also showed that more work is needed to reduce the overhead associated with the more complex data structure in order to become competitive with standard approaches.

\section{Publications}

Fattebert, J. L., R. D. Hornung, and A. M. Wissink, in press. "Finite-element approach for density functional theory calculations on locally refined meshes." J. Comp. Phys.

UCRL-JRNL-220210. 


\title{
Electro-Thermal-Mechanical Simulation Capability
}

\author{
Daniel A. White \\ 04-ERD-086
}

\begin{abstract}
This project will research and develop numerical algorithms for three-dimensional (3D) electro-thermal-mechanical (ETM) simulations and incorporate them into Lawrence Livermore's computational mechanics codes ALE3D and Diablo. A coupled 3D ETM simulation solves, in a self-consistent manner, the equations of electromagnetics (EM), heat transfer, and nonlinear mechanics. Research will include advection of EM quantities in an ALE setting, algorithms for electrical contact and slide surfaces, and EM boundary conditions. Extensive algorithm analysis and code verification will be performed to ensure the model equations are solved correctly. To validate the simulation capability, we will compare simulation results to available measured data from magnetic-flux compression experiments.
\end{abstract}

We expect to develop accurate and efficient algorithms for solving coupled ETM problems on 3D unstructured, finite-element meshes. A robust, accurate ETM simulation capability developed in this project will enable LLNL physicists and engineers to better support applications such as explosively driven magnetic-flux compressors, EM launchers, inductive heating and mixing of metals, and microelectromechanical systems.

\section{Mission Relevance}

Large-scale computer simulation is a core competency of LLNL. The ETM simulation tools developed in this project will find application in weapons systems for the stockpile stewardship mission. In addition, ETM simulation tools are needed for designing the EM launchers that have been proposed for DOE equation-of-state research and Department of Defense missile defense and artillery systems.

\section{FY06 Accomplishments and Results}

We made significant progress on advection of magnetic fields for the ALE3D application. To develop an algorithm that is accurate and preserves shock fronts and divergence of the magnetic field, we wrote a novel prototype algorithm implemented in ALE3D that scales as the square of the number of grid-points for smooth fields and employs flux correction for monotonicity. We also completed a suite of verification tests including Alfven waves, an imploding wire, and a simple flux compression generator; completed a low-rank QRalgorithm-accelerated Green's function method for air regions; began to investigate methods for sliding electrical contact; and published manuscripts in peer-reviewed journals. 


\title{
Proposed Work for FY07
}

The sliding electrical contact algorithm for Diablo will be completed. We will develop an approach for coupling the electromagnetic partial differential equations with a resistanceinductance-capacitance circuit model to more realistically model experiments driven by capacitor banks. Existing measured data for validation includes current versus time and Bfield versus time for flat-plate, coaxial, and helical generator experiments. This validation effort will provide key metrics of success. We will also discuss possible collaboration with the railgun design team at the Naval Surface Warfare Center in Dahlgren, Virginia. The team is eager to use our codes to guide their experiments, and the collaboration would provide critical validation data for this project.

\section{Publications}

Rieben, R., D. White, and B. Wallin, in press. "An arbitrary Langrangian-Eulerian discretization of MHD on 3D unstructured grids." J. Comput. Phys. UCRL-JRNL-222113.

White, D., B. Fasenfest, and M. Stowell, in press. "A parallel computer implementation of fast low-rank QR approximation of the Biot-Savart law." Progr. Electromagn. Res. UCRL-PRES-218238.

White, D., B. Fasenfest, and R. Rieben, in press. "A QR-accelerated volume-to-surface boundary condition for the finite element solution of eddy current problems." IEEE Trans. Magn. UCRL-JRNL-224344.

\section{A New "Natural Neighbor" Meshless Method for Modeling Extreme Deformations and Failure}

Michael A. Puso

04-ERD-088

\begin{abstract}
The objective of this work is to develop a fully Lagrangian approach-based on "natural neighbor" techniques-to model extreme deformation and failure for analyses such as earth penetration and dam failure. In these problems, the standard Lagrangian finiteelement approach fails due to mesh tangling, whereas Eulerian codes do not allow us to track particles and free surfaces. Meshless methods such as smooth particle hydrodynamics (SPH) and element-free Galerkin (EFG) have been used for modeling such large deformations but have a variety of numerical problems that the new natural neighbor approach can potentially solve. The work will involve research in meshless discretization and integration techniques. The meshless capability has already been added to our DYNA3D finite-element code validated using penetrator data.
\end{abstract}

If successful, the new approach will provide an improved method for modeling extreme events such as earth penetration and dam failure. For example, because the method is fully Lagrangian, better descriptions of anisotropic material damage of concrete can be 
applied; even water spilling over a damaged dam could be modeled with this approach. Being meshless, the approach could be used for applications where nondestructive evaluation is required, from weapons analysis to biomechanics. The method is more stable than smooth-particle hydrodynamics methods, faster than element-free Galerkin meshless methods, and circumvents the advection required by arbitrary Lagrangian-Eulerian techniques, making it applicable to a much larger class of problems.

\section{Mission Relevance}

The proposed approach treats both solid and fluid mechanics and is therefore applicable to several important LLNL national security and homeland security mission areas. Analyses of earth penetration (e.g., for the development of conventional munitions designed to hold deeply buried hardened targets at risk) and terrorist vulnerability evaluation of infrastructure elements (e.g., dams) are possible applications for this new approach. As-built $x$-ray tomography of laser targets and in vivo magnetic resonance imaging in biomechanics that create "point clouds" would benefit from this meshless method by expediting stress analysis.

\section{FY06 Accomplishments and Results}

In FY06, we developed a modified integration scheme that no longer requires Voronoi cells. For consistency in the general case, a special correction term was developed to solve the patch test. We also implemented standard classical meshless methods-moving least squares, reproducing kernel particle method, meshless local Petrov-Galerkin, and SPHand extended the nearest-neighbor approach to these methods by replacing spherical kernel functions with ellipsoidal kernels that fit nearest neighbors. The integration scheme and nearest neighbor modifications to classical schemes proved robust, and the new methods were successfully tested.

\section{Proposed Work for FY07}

In FY07 we will (1) continue validation of the new methods, focusing on earth penetration but looking at other experimental results, as well; (2) continue working on nodal integration correction to determine its necessity and robustness in large deformation problems; (3) look at ways to improve our method to solve the problem of pressure oscillation due to incompressibility; and (4) time permitting, add error indicators to enable point insertion and the conversion of finite elements into particles.

\section{Publications}

Chen, J. S., W. Hu, and M. A. Puso, in press. "Orbital HP-clouds for solving Schrodinger's equation in quantum mechanics." Comput. Meth. Appl. Mech. Eng. UCRL-JRNL-225453.

Puso, M. A., E. Zywicz, and J. S. Chen, 2005. "A new stabilized nodal integration approach." Proc. 3rd Intl. Workshop Mesh-free Methods for Partial Differential Equations. UCRL-PROC-218895. 


\title{
Internet Ballistics: Identifying Internet Adversaries Despite Falsified Source Addressing
}

\author{
Anthony Bartoletti 04-ERD-095
}

\begin{abstract}
This project will determine the degree to which an Internet adversary can be identified through packet timing characteristics imposed unwittingly by the attacker's choice of platform, software, algorithm settings, and network locations, with focus upon hostile scan activity. Most network security efforts require adversary identification and characterization, but rely primarily upon easily spoofed or illicit source Internet Protocol (IP) addresses. This research seeks to supplant the IP address with other evidence that is characteristic of the adversary. We will measure the influence upon packet arrival patterns from varied attack software, platform performance, and intervening network conditions by employing controlled experiments with reference platforms. Mathematical characterization will employ wavelets.
\end{abstract}

If successful, the project will be able to separate adversary-specific traffic signatures from those of network-specific traffic, and will provide a testable foundation for a system of attacker attribution that can be employed in augmenting and disambiguating adversary profiles. In the cybersecurity realm, added confidence in source attribution can lead to improved discovery of related intrusions that might have been overlooked because of deliberately obscured source-IP addresses. A precise regimen of measures best suited to feature separation and detection will be detailed. Directions for further research in improving the underlying metrics will be indicated.

\section{Mission Relevance}

This work will support Laboratory and DOE/NNSA's national security missions in cybersecurity and will benefit counterintelligence efforts by increasing analysts' ability to correlate what might otherwise appear as unrelated network activities.

\section{FY06 Accomplishments and Results}

In FY06, we conducted tests involving over 200 controlled, non-routed scans using the scanning tools Nmap, Hping2, and Strobe. Significant success was achieved in demonstrating the ability to identify attack tools and their specific, attacker-selected settings. Application of Mahalanobis and similar variance-sensitive distance functions were shown to result in significantly greater accuracy. Our results with the University of California at Davis ScanVis tool demonstrate its facility in refining our discrimination measures. Finally, ongoing controlled testing has been extended to routed (Internettraversing) scans to determine the effectiveness of our methods in distinguishing attacker network location. 


\title{
Proposed Work for FY07
}

In FY07, we plan to (1) conduct additional routed tests; (2) provide a rigorous analytical treatment of the results; (3) demonstrate the operational capability of our tools by preparing a packaged software system, complete with preliminary identification baselines and methods for continued refinement of measures; (4) prepare a paper for publication in a refereed journal; and (5) deploy this capability in support of LLNL's network security and counterintelligence efforts.

\section{Publications}

Muelder, C. W., K. Ma, and A. Bartoletti, 2005. "A visualization methodology for characterization of network scans." Proc. Workshop on Visualizations for Computer Security (VizSEC), p. 29. UCRL-CONF-217880.

\section{Petascale Simulation Initiative}

John M. May

04-ERD-102

\begin{abstract}
This project will dramatically increase LLNL's capability in scalable multiphysics and multiscale simulations for stockpile stewardship and other applications. The focus will be development of algorithms and software for efficient coupling of multiphysics calculations in a multiple-program, multiple-data (MPMD) environment on massively parallel computers (e.g., BlueGene/L) and on adaptive algorithms for multiscale modeling. The architecture developed will facilitate coupling existing, independently written codes, leading to new multiphysics and analysis capabilities. Advances to the programming environment will provide more flexible, and potentially more efficient, use of massively parallel computers at LLNL. Adaptive sampling will enable more efficient use of multiscale models.

A scalable, simulation-component ("symponent") architecture (SA) will be developed and implemented for multiphysics codes, and the core capabilities will be demonstrated with a full-scale simulation on a high-performance computing platform. Adaptive sampling algorithms will be created and tested, permitting significantly more efficient use of subscale physical models in full-scale simulations. The code coupling and adaptive sampling software will be portable, usable with other LLNL codes, and applicable to a broad class of material models. The software will take advantage of MPMD parallelism for the dynamic launching of component codes. All of the above will be demonstrated in a large-scale simulation using a detailed subscale polycrystalline material model.
\end{abstract}

\section{Mission Relevance}

This project supports stockpile stewardship, homeland security, and other mission areas that require the multiphysics, multiscale synthesis of existing models. The SA will facilitate coupling existing codes, shortening development time for complex applications. MPMD 
capabilities exercised in the demonstration problem will help achieve greater machine efficiency. Adaptive sampling applied to multiscale material modeling will be useful for many applications requiring subscale physics.

\title{
FY06 Accomplishments and Results
}

In FY06, we (1) rebuilt our runtime system, emphasizing reliability, clean interfaces and better service, scaling, and instrumentation; (2) produced a symponent for the finiteelement code ALE3D and extended it to enable launching independent processes for computing fine-scale model results; (3) improved the efficiency and accuracy of our sampling and coupling algorithms; (4) created a regression test suite; (5) extended the remote method invocation capability between symponents; and (6) ran ALE3D using adaptive sampling to model a shocked cylinder. In the demonstration, which ran with our MPMD runtime software on 680 nodes of the MCR supercomputer, we achieved a 37-fold speedup compared to the same run without adaptive sampling or MPMD programming.

\section{Proposed Work for FY07}

In FY07, we will (1) conduct extensive performance studies on our adaptive sampling technique and runtime software; (2) complete a new demonstration using a different application to exercise the job-launch capabilities of the runtime system; (3) explore additional new uses for the MPMD programming model in collaboration with other LLNL researchers; and (4) prepare and submit papers documenting our MPMD model and adaptive sampling.

\section{Publications}

Tannahill, J., P. Miller, and G. Kumfert, 2006. C++ coding guidelines for the Petascale Simulation Initiative (PSI) project. UCRL-SM-221698.

\section{Catalyzing the Adoption of Software Components}

\author{
Thomas G. Epperly 05-ERD-012
}

\begin{abstract}
We will develop technology to automate the componentization of existing source code, which will in turn improve the productivity of code developers. Software component technology has redefined state-of-the-art software engineering practice in industry, enabling codes to be larger, more modular, scalable, robust, and amenable to change. Component technology can also benefit LLNL. Our in-house expertise and tools uniquely position us to conduct research in automated componentization with more sophisticated techniques than those in the literature. By providing a lower-cost path towards componentization, we will help LLNL maintain the lead in delivering large, modular, scalable, robust, and flexible simulation codes in the pursuit of science.
\end{abstract}


This project will provide (1) technology to automate construction of components by automatically partitioning existing functions into components, (2) technology to automatically generate Babel interfaces for $\mathrm{C} / \mathrm{C}++$ software libraries and components, (3) software tools to consider multiple alternative componentizations, (4) technology to visualize large-scale applications and their decomposition into components, and (5) technology to evaluate and visualize software features to promote software comprehension.

\section{Mission Relevance}

Many Laboratory activities, including stockpile stewardship, information processing, and chemical and biological security, depend on scientific computation to further science in the national interest. This project supports many LLNL missions by providing a concrete approach to development of components within existing LLNL applications. This work will make development and maintenance of large-scale applications cheaper, more robust, and more flexible.

\section{FY06 Accomplishments and Results}

In FY06 we (1) connected the ROSE analysis engine to VizzAnalyzer-a computing environment for reverse-engineering software-to enable the presentation of multiple alternative componentizations; (2) implemented and experimented with clustering algorithms to perform case studies on small libraries; (3) tested applications on large-scale applications to find black-listed programming constructs to experiment with large-scale case studies; and (4) published a scientific paper and presented invited talks.

\section{Proposed Work for FY07}

Our focus in FY07 will be to (1) complete integration of our clustering analysis and automatic componentization transformations, (2) perform case studies on LLNL applications and libraries, (3) develop design tools to provide choices between multiple componentization schemes and the ability to manually edit the results, (4) use interface quality metrics to improve the automatically generated component interfaces, and (5) author several journal or conference papers on our results.

\section{Publications}

Panas, T., and D. Quinlan, 2006. Reverse engineering large $C / C++$ applications. UCRL-PRES-219761-DRAFT.

Quinlan, D., and T. Panas, 2006. ROSE. UCRL-PRES-219761.

Quinlan, D., R. Vuduc, and T. Panas, 2006. Support for whole-program analysis and the verification of the one-definition rule in $\mathrm{C}++$. Static Analysis Summit, Gaithersburg, MD, June 29, 2006. UCRL-CONF-222122. 


\title{
LOCAL: Locality-Optimizing Caching Algorithms and Layouts
}

\author{
Peter G. Lindstrom 05-ERD-018
}

\begin{abstract}
This project is investigating layout and compression techniques for large, unstructured simulation data to reduce latency and bandwidth requirements for visualization and related tasks. Our goal is to eliminate the data-transfer bottleneck-for example, from disk to memory and from CPU to GPU-through cache-coherent data access and by trading underutilized compute power for bandwidth and storage. We are approaching this by designing algorithms that both enforce and exploit compactness and locality in unstructured data, and by adapting offline computations to a novel stream-processing framework that supports pipelining and low-latency sequential access to compressed data. The scalable algorithms developed in this project run on both end-user desktops and dedicated visualization clusters.

We expect to achieve dramatic improvements in disk and memory usage, effective bandwidth, data-access latency, and cache reuse, which will result in more efficient random and sequential access to unstructured grid data. These improvements will lead to the ability to manage larger data sets and store more complete simulation data dumps for analysis and visualization. They will additionally provide new capabilities and orderof-magnitude performance improvements in simulation setup, offline mesh processing, interactive data queries, and real-time paging and rendering. These bandwidth- and latency-efficient techniques will become increasingly valuable on next-generation supercomputers as the gap between processor speed and input-output performance keeps widening.
\end{abstract}

\section{Mission Relevance}

Our research will serve as an important aid in managing and visualizing large data sets from scientific and engineering simulations by supporting analysis and interactive exploration of terascale data sets for the stockpile stewardship mission.

\section{FY06 Accomplishments and Results}

In FY06, we developed (1) streaming compression of tetrahedral meshes (with 40x lossless reduction), (2) floating-point compression of scalar fields integrated with LLNL's Miranda code (with $4 \times$ lossless and $20 \times$ imperceptibly lossy reduction), (3) new cache coherence metrics that generalize space-filling curves to unstructured grids, (4) metrics for measuring and optimizing the streamability of a layout, (5) spectral predictors for irregular stencils, (6) a technique for the streaming computation of Reeb graphs, (7) hierarchical layouts coherent in space and connectivity, (8) techniques for progressive volume rendering and coding of graphics primitives, and (9) scalar field representations with locality in field value. 


\section{Proposed Work for FY07}

In FY07, we plan to (1) extend streaming input-output to particles and more general meshes, multivariate data, and ghost elements (extensions that are critical for Lab applications); (2) develop representations and techniques for decomposing and recombining streams for possibly pipelined parallel processing (needed to exploit the compute power of existing visualization clusters); (3) investigate the possibility and utility of multiresolution windowed streaming; (4) design a compressor that supports random access to unstructured meshes (we expect up to $100 \times$ data reduction and muchshortened application startup times); and (5) extend cache-oblivious meshes to support multiresolution, progressive access.

\section{Publications}

Callahan, S. P., et al., 2006. "Progressive volume rendering of large unstructured grids." IEEE Trans. Visual. Comput. Graph. 12, 1307. UCRL-PROC-208680

Ibarria, L., P. Lindstrom, and J. Rossignac, 2006. Predictors for streaming compression of scalar fields. UCRL-TR-219086.

Isenburg, M., et al., 2006. Streaming compression of tetrahedral volume meshes. Graphics Interface, Quebec City, Canada, June 7-9, 2006. UCRL-CONF-217274.

Lindstrom, P., and M. Isenburg, 2006. "Fast and efficient compression of floating-point data." IEEE Trans. Visual. Comput. Graph. 12, 1245. UCRL-JRNL-220406.

Pascucci, V., G. Scorselli, and P.-T. Bremer, 2006. "Streaming computation and simplification of Reeb graphs: simplicity and speed." UCRL-JRNL-218501-DRAFT.

Vo, $\mathrm{H}_{\text {., }}$ et al., in press. "Streaming simplification of tetrahedral meshes." IEEE Trans. Visual. Comput. Graph. UCRL-JRNL-208710.

Yoon, S.-E., and D. Manocha, 2006. "Cache-efficient layouts of bounding volume hierarchies." Comput. Graph. Forum 25, 507-516. UCRL-JRNL-219070.

Yoon, S.-E., C. Lauterbach, D. Manocha, 2006. "R-LODs: fast LOD-based ray tracing of massive models." Vis. Comput. 22, 772. UCRL-JRNL-219092.

Yoon, S.-E., and P. Lindstrom, 2006. "Mesh layouts for block-based caches." IEEE Trans. Visual. Comput. Graph. 12, 1213. UCRL-JRNL-220368. 


\title{
Risk Analysis of Secure Knowledge Discovery
}

\author{
Deborah W. May 205-ERD-041
}

\begin{abstract}
We seek to quantify the risks associated with applying knowledge-discovery rule sets within a mixed-classification, knowledge-management system. Many Department of Defense and Department of Homeland Security communities rely on people to manually classify information based on nonquantitative methods. This research will formalize and automate the process of making sound classification judgments based on a quantitative risk analysis. It will also enable automated classification of algorithmically derived information to be made on a massive scale-a much needed capability for Lawrence Livermore's next-generation knowledge fusion systems. The project will quantify the risks of both too much security, by measuring knowledge fragmentation in graph-modeled information, and too little security, by measuring the potential for information leakage.

Our main goal is to develop a mathematical basis for quantifying the risks of automating security in systems. The results from this research will provide a valuable tool for engineers, scientists, and decision makers to understand the risks associated with classification policies. Using this tool, they will be able to make classification decisions based on rigorous mathematical analyses of the knowledge-sharing loss that results from overly stringent security policies, and the risk of information leakage resulting from overly lenient security policies. The tool also offers the user technologies that will maximize both security and knowledge sharing. In addition, this project will enable automated classification of information discovered or extracted by computer algorithms.
\end{abstract}

\section{Mission Relevance}

This project will provide the science base for the Laboratory to develop sound information security policies, secure knowledge-discovery rule sets, and robust information-to-insight applications that support information systems for a wide range of national security and homeland security missions.

\section{FY06 Accomplishments and Results}

In FY06, we developed a unique method, "subgraph matching," to determine the entropy of most-likely statistics graphs, when data is excluded for classification purposes. To accomplish this, we developed an entropy testing engine that can utilize synthetic or real graphs to test the subgraph matching approach, and have submitted results of our work to a peer-reviewed journal. Overall, we have developed the base necessary to create a costbenefit software tool that examines tradeoffs between security and sharing in classifying information. 


\title{
A New Method for Wave Propagation in Elastic Media
}

\author{
Anders Petersson 05-ERD-079
}

\begin{abstract}
Simulation of elastic-wave-propagation is essential for monitoring nuclear explosions, prediction of ground motion from earthquakes, and nondestructive evaluation of complex parts. This project is developing significant improvements of the traditional finitedifference technique that will allow a fully second-order accurate treatment of boundary conditions in complex domains to handle topography and internal layers, and that will use local mesh refinement to avoid partial oversampling of the solution because of varying wave speeds. The boundary conditions will be implemented using new embedded boundary technology for wave equations in second-order formulation. Adaptive meshrefinement techniques from compressible fluid mechanics will be generalized to suit the elastic wave equation.
\end{abstract}

This project will result in a verified, accurate, and efficient elastic-wave-propagation code for numerical simulation in complex two- and three-dimensional (2D and 3D) media. This open-source code will be used for many applications at LLNL and in the scientific community. The computer software will support applications ranging from 2D simulations in nondestructive testing to 3D earthquake modeling, using computer power ranging from desktop workstations (e.g., SUN, LINUX, and OSX platforms) to massively parallel high-performance machines (e.g., the Multiprogrammatic Capability Resource machine, Thunder, and Atlas). We plan to validate the code against benchmark problems relevant to Laboratory program applications, and publish our research in journals and conference proceedings.

\section{Mission Relevance}

Simulation of seismic-wave-propagation phenomena is essential for the success of many applications in support of Lawrence Livermore's national security mission. This includes strong ground-motion prediction for the Enhanced Test Site Readiness Program and the Yucca Mountain Program, nuclear explosion monitoring and underground facilities characterization, and nondestructive evaluation for locating imperfections in critical components relevant to the Transformational Materials Initiative.

\section{FY06 Accomplishments and Results}

Our FY06 efforts focused on large-scale simulations of the 1906 San Francisco earthquake as a means of validating our code. We developed the capability to (1) read the geologic model provided by the U.S. Geological Survey, (2) perform robust discretization of rapidly fluctuating material properties, (3) extend the elastic scheme to allow for acoustic regions (water), (4) improve discretization of moment sources to allow a general 3D fault surface, (5) remove solid-body motion using a projection technique, and (6) interface a wavelet library for compressing volumetric data for 3D post-processing. 


\section{Proposed Work for FY07}

In FY07, we will (1) complete theoretical development of the embedded boundary technique for enforcing stress-free boundary conditions on voids and topography with complex shapes, (2) develop patch-based local mesh refinement to allow the mesh size to approximately follow the velocity structure in the computational domain, (3) implement these developments into the Wave Propagation Program code, (4) validate simulations with free surface topography against a spectral element method code running at the Laboratory, and (5) port the Wave Propagation Program code to other platforms to broaden the user base and exercise the code in new ways.

\section{Publications}

Nilsson, S., 2006. A new finite-difference method for elastic wave propagation with applications in seismology. 7th World Congress on Computational Mechanics, Los Angeles, CA, July 16-22, 2006. UCRL-ABS-219084.

Nilsson, S., 2006. Seismic sources and waves using finite differences. Society for Industrial and Applied Mathematics Ann. Mtg., Boston, MA, July 10-14, 2006. UCRL-ABS-218316.

Nilsson, S., et al., in press. "Accurate and stable finite difference approximations of the elastodynamic equations in second order formulation." J. Comput. Phys.

UCRL-JRNL-218514.

Nilsson, S., et al., 2006. A new finite-difference method for seismic applications.

Seismological Society of America, San Francisco, CA, Apr. 18-22, 2006.

UCRL-ABS-218127.

Petersson, A., et al., 2006. Large scale seismic modeling at LLNL. Ann. Northern California Earthquake Hazards Workshop, Menlo Park, CA, Jan. 18-19, 2006. UCRL-ABS-218102.

Petersson, A., et al., 2006. Large scale seismic modeling of the great 1906 San Francisco earthquake. Seismological Society of America, San Francisco, CA, Apr. 18-22, 2006.

UCRL-ABS-218213.

Petersson, A, et al., 2006. "A new method for wave propagation in elastic media." UCRL-PRES-223206.

Rodgers, A., et al., 2006. Ground motion predictions using the USGS seismic velocity model of the San Francisco Bay area. Seismological Society of America, San Francisco, CA, Apr. 18-22, 2006. UCRL-ABS-218134.

Rodgers, A., et al., 2006. Regional and global scale modeling of the great 1906 San Francisco earthquake. Seismological Society of America, San Francisco, CA, Apr. 18-22, 2006. UCRL-ABS-218066. 


\title{
Efficient and Reliable Data Exploration via Multiscale Morse Analysis and Combinatorial Information Visualization
}

\author{
Valerio Pascucci 05-ERI-002
}

\begin{abstract}
We propose to develop a new visualization framework based on general-purpose dataanalysis tools coupled with information-visualization techniques. The framework will allow fast computation and effective display of metadata roadmaps guiding the exploration of terabytes of raw data. We are using Morse analysis to build multiscale models of fundamental structures that are ubiquitous in scientific data. The large size and complexity of the topology graphs obtained will require new and general multiscale graph models that we will apply to Web security graphs and counterproliferation semantic graphs. The environment will use linked views to show the graphs with context information that improves the overall data understanding.
\end{abstract}

The success of this project will yield new data-exploration modalities for smooth and discrete data. At the scientific level, this will contribute new basic research both in information visualization and in topology-based data analysis. In these areas we will develop new multiresolution representation models and external memory algorithms and data structures. On the practical level, our technology will allow us to develop tools for data analysis and presentation that will improve the effective speed of accessing the information stored in terascale scientific data sets and in large semantic graphs. This will be accomplished both by increasing performance of the display methods and by integrating multiple presentation modalities for improved data understanding.

\section{Mission Relevance}

This research will enable new techniques for analysis and visualization of terascale scientific data of the type generated by LLNL's stockpile stewardship program, such as large-scale simulations of hydrodynamic instabilities in shocked materials or material defect simulations with billions of atoms. We target the needs of scientists to explore such datasets with confidence that important features are not overlooked.

\section{FY06 Accomplishments and Results}

In FY06 we extended many two-dimensional (2D) techniques to the volumetric case, completed a number of robust algorithms for 3D topology computation (including a 3D Morse complex), and started to test simplification of the Jacobi sets over time. This created the capability to (1) use topological analysis to segment bubble structures in mixing-fluid dynamics simulations, (2) use time tracking to provide detailed information and global summaries describing turbulent mixing behavior, (3) integrate the topology components with the visualization framework based on linked views, and (4) extend the tree-visualization tools to allow general graphs with cycles. 


\section{Proposed Work for FY07}

In FY07 we will (1) complete the 3D topology computation scheme with explicit simplification of selected topological features, (2) simplify critical points in selected time steps of a dynamic model using Jacobi sets, (3) finish scaling the algorithms to large data, (4) complete the out-of-core progressive data structures and algorithms for presentation of semantic graphs, (5) deploy the tools as libraries that can be easily integrated in external systems such as the VisIT visualization system, and (6) collect practical models of specific relevance for verification and validation and demonstrate to users our approach to quantitative analysis and data comparison.

\section{Publications}

Bethel, W., et al., 2006. "Vacet: proposed SciDAC2 visualization and analytics center for enabling technologies." J. Phys.: Conf. Ser. 46, 561-569. UCRL-JRNL-208710.

Bremer, B.-T., and V. Pascucci, in press. "A practical approach to two-dimensional scalar topology." Topology-Based Methods in Visualization. UCRL-BOOK-218178.

Bremer, P.-T., et al., 2006. "On the analysis of the turbulent mixing layer in hydrodynamic instabilities." J. Phys.: Conf. Ser. 46, 556-560. UCRL-JRNL-208710.

Callahan, S. P., et al., in press. "Progressive volume rendering of large unstructured grids." IEEE Trans. Visual Comput. Graph. UCRL-JRNL-208710.

Dillard, S. E., et al., 2006. Tessellation of quadratic elements. 17th International Symp. Algorithms and Computation, Kolkata, India, Dec. 18-20, 2006. UCRL-JRNL-208710.

Dong, S., et al., 2006. "Spectral surface quadrangulation." ACM Transactions on Computer Graphics 25, 1057-1066. UCRL-JRNL-218500.

Edelsbrunner, H., D. Morozov, and V. Pascucci, 2006. "Persistence-sensitive simplification functions on 2-manifolds." ACM Symp. Computational Geometry 22, 127-134.

UCRL-PROC-220451.

Gyulassy, A., et al., 2005. "Topology-based simplification for feature extraction from 3-D scalar fields." IEEE Visual. Conf. 1, 535-542. UCRL-PROC-216195.

Gyulassy, A., et al., in press. "A topological approach to simplification of three-dimensional scalar functions." IEEE Trans. Visual Comput. Graph. UCRL-JRNL-208700.

Laney, D., et al., in press. "Understanding the structure of the turbulent mixing layer in hydrodynamic instabilities." IEEE Trans. Visual Comput. Graph. UCRL-JRNL-208710.

Natarajan, V., et al., 2006. "Segmenting molecular surfaces." Comput. Aided Geomet Des. 23, 495-509. UCRL-JRNL-218500. 
Vo, H., et al., in press. "Streaming simplification of tetrahedral meshes." IEEE Trans. Visual Comput. Graph. UCRL-JRNL-208710.

Weber, G. H., et al., in press. "Topology-controlled volume rendering." IEEE Trans. Visual Comput. Graph. UCRL-JRNL-208710.

\section{Predictive Knowledge Systems for Large, Complex Data Sources}

James Brase

06-SI-006

\section{Project Description}

Nonproliferation, counterterrorism, and intelligence are primarily problems of information: Sensors and data-collection systems can provide overwhelming quantities of data. Moreover, these data are often sparse, noisy, irrelevant, and even intentionally misleading. The objective of this project is to discover complex patterns in large-scale multisource data streams and to build predictive models based on these patterns. We will create algorithms and computations capabilities that allow analysts to extract knowledge from such data in a meaningful and timely way. To this end, we will pursue pattern discovery, learning, and prediction implemented in data-intensive computational architectures. The project will focus on demonstrating applications in nonproliferation and homeland security.

We expect to develop (1) a technical base of algorithms and computational methods that will extend the frontiers of pattern recognition and stochastic predictive models,

(2) confidence measures and performance metrics relevant to these tools, and (3) a series of prototype demonstrations that apply these capabilities to important problems of nuclear nonproliferation and homeland security.

\section{Mission Relevance}

We will apply these capabilities to draw accurate and relevant conclusions from a mix of complex data sources (e.g., imagery, sensor networks, semantic graphs, and relational databases) in order to demonstrate unprecedented levels of performance in broad-area search and site-monitoring problems relevant to nonproliferation and homeland security. The central core of algorithms and computational methods developed will broadly support LLNL's missions in national security, particularly nonproliferation and homeland security.

\section{FY06 Accomplishments and Results}

In FY06, we (1) established research and programmatic databases for communication, text, and imagery; (2) designed a simulation tool for generating data sets with controlled signatures and backgrounds in a weapons-of-mass-destruction nonproliferation scenario; (3) demonstrated road network detection algorithms needed to evaluate spatial likelihood maps of transportation networks; (4) demonstrated vehicle-tracking algorithms for persistent surveillance imagery and demonstrated performance for Sonoma; (5) established an operational graph research testbed, developed an applications programming interface for determining patterns in communication traffic data, and 
demonstrated temporal feature extraction from the research communication database; and (6) designed an integrated data management system combining semantic graphs with a geographic information system (GIS).

\section{Proposed Work for FY07}

In FY07, we propose to (1) fully implement the scenario data set generator; (2) demonstrate importing of a dynamic data set into the semantic graph-GIS data management system; (3) evaluate a multimodal query with both graph and spatial components and conduct a demonstration in a contextual vehicle search; (4) develop baseline tools for discovering and classifying patterns in dynamic graphs and apply the tools to selected scenario and program data sets; (5) develop baseline activity-modeling tools and demonstrate state estimation in a scenario data set; and (6) evaluate scaling performance of algorithms and evaluate mapping to scalable architectures.

\section{Publications}

Glaser, R. E., et al., in press. "A Markov chain Monte Carlo-based method for flaw detection in beams." J. Eng. Mech. UCRL-JRNL-225176.

Manay, S., and D. W. Paglieroni, 2007. Matching flexible polygons to fields of corners extracted from images. UCRL-CONF-226354.

Paglieroni, D. W., and S. Manay, 2007. Signal-to-noise behavior of gradient direction models for corner detection in images. SPIE Defense and Security Symp., Apr. 9-13, 2007, Orlando, FL. UCRL-ABS-224478.

Paglieroni, D. W., and F. Nekoogar, 2007. Matching random tree models of spatio-temporal patterns to tables or graphs. IEEE Symp. Computational Intelligence and Data Mining, Apr. 1-5, 2007, Honolulu, HI. UCRL-CONF-225685.

\section{Development of Computational Techniques for Decoding the Language of Genomes}

Ivan V. Ovcharenko 06-ERD-004

\section{Project Description}

We will develop computational methods and tools to decipher the universal language of gene regulation encoded in genomes. The study will consist of identifying and deciphering the code and functionally annotating genomic elements that orchestrate the temporal and spatial dynamics of gene expression in living cells. Currently, no means exists to annotate pathways of protein-DNA interactions, predict biological response to environmental conditions, or explain dynamics of developmental processes on a genome scale. The proposed study will provide the means to tackle these problems computationally and will produce results with immediate practical use in a variety of experimental studies across the Laboratory. 
We will develop computational techniques to decipher the gene-regulation code in genomes. Specifically, we will (1) produce a generalized method to identify regulatory elements (REs) in genomes; (2) establish associative rules of genome sequence with the dynamics of genome biological activity; (3) develop a database of REs in genomes; (4) develop genome scans for REs with a particular biological function; (5) annotate gene regulatory networks in the human genome; (6) develop strategies to dissect gene regulation from microarray experiments; and (7) expand the existing toolkit for comparative genomics analysis.

\section{Mission Relevance}

This project supports LLNL's mission in bioscience and technology to improve human health by establishing novel technologies for breaking the code of gene regulation. These results in computational genomics will also be directly relevant to efforts in environmental biology and remediation.

\section{FY06 Accomplishments and Results}

After a midyear start, we (1) combined experimental gene-expression profiling and heuristic sequence analysis to define signatures of regulatory elements in complex genomes, (2) developed a computational method for de novo identification of functional elements in genomes, (3) demonstrated that this unique method performs exceptionally well in blind detection of experimentally characterized mouse enhancer elements, and (4) published manuscripts on our work.

\section{Publications}

Loots, G. G., and I. Ovcharenko, in press. "ECRbase: database of evolutionary conserved regions, promoters, and transcription factor binding sites in vertebrate genomes." Bioinformatics. UCRL-JRNL-223593.

Loots, G. G., et al., 2006. "Array2BIO: from microarray expression data to functional annotation of co-regulated genes." BMC Bioinformatics 7, 307. UCRL-JRNL-219041.

Pennacchio, L. A., et al. Genome-wide de novo prediction of proximal and distal tissuespecific enhancers. UCRL-JRNL-216861.

\section{Scalable Data Management for Massive Semantic Graphs}

Scott R. Kohn

06-ERD-009

\section{Abstract}

Semantic graphs are an important tool for the intelligence community and homeland security applications. However, current approaches do not address anticipated data sizes. 
We are investigating scalable parallel approaches for semantic graphs that are 100 times larger than currently possible. We are creating a prototype, parallel semantic-graph engine that ingests graph data at high speed, distributes and blocks edge data, and executes graph queries across hundreds of parallel processors. Our approach is unique in that we distribute the semantic graph data across the processors of a parallel computing cluster. Our research focuses on the algorithms and techniques necessary to support massive distributed semantic graphs.

The goal of this research is to understand how to use distributed-memory computing clusters to support semantic graphs that are orders of magnitude larger than those supported with current nondistributed approaches. We expect this research to guide the development of next-generation, semantic-graph architectures. Our semantic-graph performance model and cluster ideas will be submitted for publication in peer-reviewed journals.

\section{Mission Relevance}

Increasingly massive data sets collected for nonproliferation, intelligence, and military missions require new technologies to query and analyze these data. By furthering understanding performance characteristics of a cluster architecture on data-intensive semantic graph applications, this project supports the large-scale data management and analysis needs of Lawrence Livermore's homeland and national security missions.

\section{FY06 Accomplishments and Results}

In FY06, we (1) demonstrated a serial semantic graph system that ingests and fuses graph data, and began making progress towards a scalable parallel system; (2) developed a parallelization approach that employs a data-decomposition strategy based on cryptographic hashes and static processor assignment that does not require interprocessor synchronization on ingest; (3) developed new edge-partitioning algorithms and investigated efficient out-of-core representations for storing edges for graph search; (4) demonstrated that we can achieve a fivefold improvement in search performance by blocking edges using only local graph information; and (5) defined a graph query language for parallel execution, and began implementing that language.

\section{Proposed Work for FY07}

In FY07, we will (1) demonstrate parallel ingestion of data by studying the scalability of our approach with increasing numbers of processors and investigating the performance tradeoffs of various attribute-decomposition strategies; (2) complete implementation of the query engine for nonpattern graphs, demonstrating parallel queries for a semantic graph distributed over multiple processors and studying the impact of various attributedecomposition strategies on query performance; (3) improve the out-of-core data representation of our SGRACE streaming-edge partitioner to enable efficient bulk loading and access to edges; and (4) extend SGRACE to run on multiple processors to increase performance. 


\title{
Publications
}

Hartley, T., et al., 2006. Mssg: a framework for massive-scale semantic graphs. Cluster 2006 Conference, Barcelona, Catalonia, Spain, Sept. 25-28, 2006. UCRL-PROC-222334.

Kaplan, I., 2006. Notes on a graph query language for a parallel graph system. UCRL-TR-225447-DRAFT.

\section{A Predictive Model of Fragmentation Using Adaptive Mesh Refinement and Hierarchial Material Model}

Alice E. Koniges 06-ERD-036

\begin{abstract}
Fragmentation in materials naturally spans microscopic to macroscopic scales. Recent advances in algorithms and computer power will enable us to connect the continuum to microstructural regimes in a real simulation through a heterogeneous multiscale mathematical model. We will develop a mathematical framework using an innovative combination of hierarchical material modeling (HMM) and adaptive mesh refinement (AMR) and apply it to a problem that can be verified experimentally and computationally with nearly full-resolution simulations on BlueGene/L-class computers. We will guide and verify these models with laser experiments and microstructural analysis. This project takes advantage of a unique opportunity for close collaboration between researchers at UC San Diego (experts in HMM) and LLNL (experts in AMR and experiments).

This project will bolster the use of the structured AMR application infrastructure (SAMRAI) library for multiscale simulations. Design decisions relating to fusion-class lasers rely on accurate prediction of fragmentation in the target chamber. This research will develop the first computationally based models for the fragmentation process that can be directly applied to fusion-class laser design issues. The predictive model developed in the project, along with the experimental component, are expected to yield an increased understanding of fragmentation, which will affect many different fields, from fusion-class lasers, to weapons systems, to space shuttle re-entry.
\end{abstract}

\section{Mission Relevance}

This research has direct relevance to national security as well as to breakthroughs in fundamental science and technology. Benefits include significant enhancement and broadening of our expertise in computational solid mechanics and advancement of the application base for our AMR framework. A significantly improved capability to predict fragmentation will find application in fusion-class laser experiments in support of the stockpile stewardship mission. 


\title{
FY06 Accomplishments and Results
}

In FY06, we (1) evaluated faceted yield surfaces and implemented a single-crystal plasticity model in a test-bed code; (2) performed initial scaling studies; (3) defined arbitrary Lagrangian-Eulerian- (ALE-) AMR algorithms and improvements necessary for inclusion of the hierarchical material model; (4) implemented two-dimensional (2D) algorithms for material interface reconstruction and strength in the Lagrangian mode of the code and began the same for the AMR mode; (5) started 3D interface reconstruction; (6) began implementing single-group radiation diffusion, with test problems on the Cartesian mesh version of ALE-AMR; (7) performed the first set of experiments and began data analysis of aerogel and glass collection plates; and (8) started implementing energy deposition in the code.

\section{Proposed Work for FY07}

We will (1) implement single-crystal plasticity models; (2) define improvements to the ALE-AMR algorithms necessary for consistent inclusion of these models; (3) continue implementing an energy-deposition package to model the laser experiments; (4) finish implementing the radiation diffusion solver and couple it with the hydro solver;

(5) address issues related to implementing the HMMs into the ALE-AMR framework and the associated refinement and coarsening operators in a scalable fashion; and (6) design and field additional experiments that will ultimately be used to benchmark the simulations.

\section{Publications}

Koniges, A. E., et al., 2005. "Modeling NIF experimental designs with adaptive mesh refinement and Lagrangian hydrodynamics." J. Phys. IV France 133, 587.

UCRL-CONF-217824.

\section{Decomposition of Large-Scale Semantic Graphs via an Efficient Communities Algorithm}

Yiming Yao 06-ERD-038

\begin{abstract}
LLNL has been developing semantic graph technologies to fuse disparate facts into massive semantic graphs for intelligence analysis. A new challenge is to decompose these massive graphs into meaningful subgraphs ("communities") for effective analysis. We are developing a new, two-stage approach for finding communities in a graph. The graph is initially transformed into an isomorphic tree of components via computationally inexpensive algorithms. A community partitioning algorithm is then applied to each of
\end{abstract}


these components separately, while accounting for the influence of the other components. This approach can achieve high efficiency because the expensive community partitioning algorithm is applied to individual components of the graph separately, resulting in parallelism.

We will deliver a software package for decomposing semantic graphs of unprecedented scale and two research papers for refereed publication. The software will constitute a new technology enabling efficient knowledge extraction and inference activities for the intelligence community and the Department of Homeland Security. The research papers will be valuable contributions to the state of the art in graph theoretic algorithms.

\section{Mission Relevance}

Algorithms to be developed in this project will lead to effective knowledge discovery systems for the Laboratory's national security mission to counter the proliferation and use of weapons of mass destruction and will support the homeland security mission by enabling more effective knowledge extraction and inference activities. In addition, by making contributions to knowledge of graphing theoretic algorithms, the project also supports the Laboratory's mission in breakthroughs in fundamental science and technology.

\section{FY06 Accomplishments and Results}

In FY06 we (1) developed algorithms for decomposing a graph into a tree of two connected components, (2) explored the partitioning of graphs into three connected components, (3) explored large semantic graphs, (4) modified Girvan and Newman's algorithm, (5) developed an optimization model for finding the best community partitioning as measured by Newman's modularity, (6) applied ideas from our research to solve a graph-query problem, and (7) presented our research findings at a professional conference.

\section{Proposed Work for FY07}

In FY07 we will (1) write parallel algorithms for component decomposition; (2) write parallel algorithms for finding communities; (3) integrate, test, and tune the algorithms; (4) conduct experiments and graph analysis; and (5) write a research paper on finding communities in graphs.

\section{Publications}

Yao, Y., 2006. Finding paths in social networks. Risk Symposium 2006, Santa Fe, NM, Mar. 20-22, 2006. UCRL-ABS-219176. 


\title{
Image Relational Search Engine
}

\author{
David W. Paglieroni 06-ERD-047
}

\begin{abstract}
One vital goal of information science is to develop a multistep process for transforming raw data into knowledge. Unformatted raw data must first be transformed into formatted tables or semantic graphs containing items of interest extracted from the data, which then must be extracted from these tables or graphs, or naturally occurring patterns discovered. This project addresses one voluminous raw data source: imagery from remote sensors. The focus is limited to extracting items of interest from images (roads, buildings, vehicles, etc.) and then searching for patterns that contain combinations of items that relate to each other in a prescribed way.
\end{abstract}

If successful, this project will dramatically increase the image search speed by enabling (1) simultaneous searches over large sets of images, and improved relevance of search results; (2) searches for entities and events that have unusual or complex relationships in space and time; and (3) fusion of information from geographic information systems and overhead images. These achievements will make it possible for image and all-source intelligence analysts to analyze massive volumes of imagery in mission-critical time frames and to discover previously undiscovered or unsuspected relationships.

\section{Mission Relevance}

This project will provide new international assessment tools that can be used to search satellite images, in support of national security missions. It will provide object population data for characterizing activities at specific sites monitored over extended periods of time. These data will provide key inputs to proposed proliferation monitoring programs, as well as national geospatial intelligence gathering. Applications of this search engine will extend to defects in high-power laser optics and weapons tomography images for the stockpile stewardship mission.

\section{FY06 Accomplishments and Results}

In FY06, we developed algorithms for extracting roads and corners of buildings from images. This enables us to locate buildings regardless of image position, size, or orientation, by finding groups of corners that satisfy rectangular shape constraints. In addition, we developed an algorithm for matching probabilistic relational models to databases and graphs. Our method is unique in that it simultaneously accounts for (1) the relative significance (importance) of each concept in the pattern model, (2) the similarity between corresponding concepts in the pattern match and pattern model, (3) the degree of relational consistency between the pattern match and pattern model, (4) probabilistic uncertainty associated with the pattern model, and (5) links missing from the pattern match. 


\title{
Data Intensive Computing
}

\author{
Ghaleb M. Abdulla \\ 06-ERD-058
}

\begin{abstract}
We propose to investigate the research issues underlying the application of highperformance computing resources to the challenges of data-intensive computing, such as processing massive amounts of data generated by sensors. We will explore these issues through three targeted case studies related to increasingly important applications: text processing, streaming-image feature extraction, and processing of streaming sensor data. One of the major challenges our research will address is the development of a computing paradigm that supports efficient spatial and temporal data management. The ultimate goal of this project is to develop scalable spatial and temporal data access algorithms that enable efficient data analysis and knowledge extraction.
\end{abstract}

We will (1) determine the requirements of emerging data-intensive applications in the areas of text, image, sensor, and graph data; (2) gain insights into the high-performance computing requirements (e.g., degree of parallelism and data parallelism vs. task parallelism) for each of the four application areas; and (3) define a conceptual architecture that is capable of running the different types of applications while sharing data, which in turn will facilitate data integration and knowledge extraction. This project will help us identify computing models and architectures that can abstract the computing requirements for these different application areas.

\section{Mission Relevance}

By developing high-performance computing capabilities for text, image, graph, and sensor data processing for applications such as processing data from radiation-detection sensors, this project supports LLNL's national security mission in intelligence, defense, and homeland security.

\section{FY06 Accomplishments and Results}

We (1) developed a spatial indexing algorithm based on that used in the SAILS library network; (2) developed an implementation of the algorithm that supports point indexing and distributed indexes, scales linearly with the number of data points, and grows exponentially using a standard database-management system; (3) implemented an incremental clustering algorithm for spatial objects and characterized query performance; (4) wrote an efficient text-indexing algorithm that supports efficient disk access while compressing indexed data; (5) used the algorithm to fully construct an index for a 4-gigabyte collection of 750,000 Usenet articles on a Linux workstation in only 50 minutes; and (6) compared our indexing results to the official results of the Text Retrieval Conference, which showed our technology to be equivalent to the best published algorithm. 


\title{
A Novel Structure-Driven Approach to Sequence Pattern Definition for Remote Homology Detection
}

\author{
Carol E. Zhou 06-ERD-059
}

\begin{abstract}
We propose to design, develop, test, and demonstrate an algorithm for detecting remote protein homology-an important need in protein-structure modeling, functional assignment, sequence variability, and the rational design of diagnostics, therapeutics, and vaccines. We will devise sequence patterns representing structure fragments and test if the patterns can detect known family members in the Protein Databank (PDB), then use patterns to predict structures for up to 200 Yersinia pestis virulence-associated proteins. We will use laboratory methods to validate our predictions and refine the algorithm.

We will devise an algorithm for automatically generating sequence patterns that embody essential protein structure information. This will immediately impact characterization of virulence proteins from one of the most important biothreat agents, $Y$. pestis (plague). A longer-term application of our algorithm is three-dimensional modeling of proteins from all pathogens of interest in biodefense. Most significantly, this would lead to more effective signatures for detection of biothreat agents (including toxins), and would yield a tool for elucidating pathogen protein function and hence, mechanisms of fitness and pathogenicity. The algorithm also will be of general use for characterization of proteins in any organism for which there is little or no structural or functional information available.
\end{abstract}

\section{Mission Relevance}

Our work supports Lawrence Livermore's mission in national security by establishing a computational capability with biodefense applications in (1) detecting and characterizing virulence proteins, (2) constructing a pathogen protein phylogeny, (3) analyzing sequence variability in rapidly evolving virus genomes, and (4) forming the rational design of diagnostics, therapeutics, and vaccines.

\section{FY06 Accomplishments and Results}

In FY06, we (1) began algorithm development, selecting several structural classification of proteins (SCOP) families; (2) structurally aligned the proteins, determined structurally conserved regions, and extracted sequence patterns using position-specific scoring matrix and hidden Markov model techniques; (3) tested our patterns against PDB and SCOP structures and nonredundant sequences, and evaluated the results; (4) expanded the $Y$. pestis virulence-associated gene set to around 500, and using in-house tools, categorized these based on sequence identity to existing structure templates; (5) built a database to organize gene information, hypothesize function, and track validation data; (6) cloned 42 genes, acquired 150 more via collaborators, and sequence-verified 126 genes of the combined set; and (7) expression tested many of the 42 clones. 


\title{
Proposed Work for FY07
}

In FY07, we expect to (1) complete development of an algorithm for automated sequencepattern extraction, (2) complete testing of the algorithm against the SCOP and PDB databases, (3) begin developing a mutation mechanism to enable detection (prediction) of remote homologs in the nonredundant sequence database, (4) complete informatics analyses toward functional assignment for the $Y$. pestis virulence-associated proteins, (5) complete cloning attempts for most of the proteins, (6) begin x-ray crystallography for up to two proteins, and (7) complete functional verification of up to two proteins. By year's end, we expect to have an algorithm that can detect remote homology, albeit with potentially high false positive and negative rates.

\section{Multi-Petabyte Image Data Management Systems}

Donald D. Dossa 06-ERD-060

\begin{abstract}
The Large-Area Synoptic Survey Telescope (LSST) will provide the most detailed map of the distribution of dark matter in the universe. The multiple petabytes of data generated will provide insights into the nature of dark energy and its impact on the evolution of the universe. Thousands of transient events-such as supernovae and gamma ray bursters-will be detected and alerts generated automatically. As a byproduct of this all-sky survey, more than $90 \%$ of the remaining unknown near-Earth asteroids will be detected and their orbits determined. This project will explore data-management issues for LSST-generated multi-petabyte data sets, including (1) optimal layout of the database and astronomical catalogs, (2) the workflow language and tools needed to combine all aspects of the astronomical image-analysis algorithms, and (3) definition of the distributed intercontinental computing and networking infrastructure spread between the telescope and base camp in the host country and the data archive and data mirror sites in the United States.
\end{abstract}

This project will deliver a detailed analysis and model that defines the data-management architecture and computing infrastructure requirements for mapping out the distribution of dark matter and achieving other goals of processing the massive data sets generated by the LSST.

\section{Mission Relevance}

The information technology to be developed for the LSST are directly applicable to the challenge of predictive knowledge management, such as near-real-time image analysis and automatically generated alerts. These capabilities will have applications in detecting changes and significant events in other countries and in quickly searching large image databases to identify terrorists or other persons of interest. Spatial and temporal indexing of petabyte databases are also needed to quickly diagnose beam characteristics of future fusion-class lasers. Consequently, this project supports LLNL's national security and homeland security missions. 


\title{
FY06 Accomplishments and Results
}

We (1) conducted research on database layouts for the high-speed searching of image data; (2) examined several workflow tools using existing astronomical image-processing codes to determine how flexibly each tool adapts to sudden changes in software components; (3) designed a detailed supercomputing system capable of meeting strict real-time requirements; and (4) ran a full image-analysis code on a Linux cluster and ported the code to parallel field-programmable gate-array accelerator modules, reaching an understanding of the performance advantages of accelerator modules and the programmer effort needed to use accelerator modules effectively.

\section{Quantum Monte Carlo Assessment of the Relevance of Electronic Correlations in Defects and Equations of State in Metals}

\author{
Andrew J. Williamson 06-LW-024
}

\begin{abstract}
We propose to develop accurate quantum Monte Carlo (QMC) capabilities to calculate defect formation energies and equations of state (EOS) of metallic systems. We will determine, for the first time in metals, the significance of electronic correlation effects in the EOS and metal-insulator transitions, and the effects on formation energies of point defects, impurities, surfaces, and interfaces beyond Density Functional Theory (DFT). These parameters determine the mechanical properties and microstructural evolution of metals under extreme conditions. Technical limitations have previously restricted application of QMC to semiconductors, insulators, and homogeneous electron gas. We have recently overcome the largest obstacle with a new formulation based on optimized, nonorthogonal orbitals.
\end{abstract}

We are developing the method and software to generate nonorthogonal transformations for real metals as an input to the CASINO QMC code. QMC values for the total formation energies of bulk aluminum will be obtained, and we will calculate some simple defects such as vacancies or surfaces for the same system. In the longer term, we will generate a formation energy table for a long list of defect structures for different materials. Under extreme pressures, when accurate experimental measurements become exceedingly difficult, tables of this sort provide data for fitting empirical interatomic potentials and continuum models. Until now, less-accurate DFT calculations have been used for this purpose.

\section{Mission Relevance}

The calculations associated with this research, which take advantage of LLNL's unique supercomputing capabilities, are key to describing matter at extreme regimes for stockpile stewardship and assessment. 


\section{FY06 Accomplishments and Results}

In FY06, we (1) generated trial wave functions for metallic systems; (2) tested the applicability of our approach by performing convergence tests using the algorithm to assess the cutoff radius of localized orbitals in a homogeneous electron gas at densities required for realistic metallic systems; (3) completed a parallel code to calculate nonorthogonal localized orbitals and to perform a DFT calculation of aluminum, using a large number of points in momentum space (k-points) to accurately represent the Fermi surface; (4) determined the optimal transformation of all the bands at all these k-points to generate a set of nonorthogonal, localized orbitals; and (5) performed the first application of this method to determine the equation of state for aluminum.

\section{Proposed Work for FY07}

In FY07 we propose to (1) extend the range of metallic systems beyond the aluminum system studied in FY06 to include beryllium, lithium, and sodium; (2) calculate the cohesive energies and formation energies of vacancies and interstitial defects in these metallic systems; (3) compute the total QMC energies based on trial wave functions originating from local-density approximation calculations that include onsite electron Coulomb interaction in a Hubbard model (if successful, this method will serve as the first parameter-free, variational total-energy technique that can describe the metal-insulator transition, beyond the mean field, for realistic electronic systems); and (4) adapt this QMC code to run on the Blue Gene/L supercomputer at LLNL.

\section{Diffusion Monte Carlo without All the Hops}

Vasily V. Bulatov

$$
\text { 06-LW-028 }
$$

\footnotetext{
Abstract

This project proposes to develop a new general method for diffusion Monte Carlo (DMC) simulations, based on the theory of first-passage processes, which will be applicable to a wide variety of conditions where the standard DMC is ineffective-e.g., when the density of diffusing particles is low and/or the distribution of their diffusion rates is wide. Our approach will boost the performance of DMC simulations by orders of magnitude, by skipping most of the diffusion hops and projecting the system's state directly to collisions, all the while leaving the statistics of random walks unaltered. We will implement the new method in an efficient computer code (both sequential and parallel versions) convenient for a wide variety of applications relevant to the Laboratory missions.

A successful completion of this project will result in a new, efficient methodology for DMC simulations applicable to a wide variety of conditions encountered in physics, chemistry, biology, and materials sciences. The new approach will overcome an ageold limitation of the standard DMC approach and open new opportunities for predictive modeling of complex processes relevant to Laboratory missions. The new development
} 
will be implemented in an efficient parallel code with user-definable local rules, rate constants, and simulation geometries in one, two, and three dimensions (1D, 2D, and 3D). The code will be maintained as an open-source software and made available to computational scientists at the Laboratory and beyond.

\section{Mission Relevance}

DMC simulations can be applied to stockpile science and technology (e.g., predictive modeling of Pu aging), to energy science and security (e.g., materials for fusion reactors and nuclear waste management), and to breakthroughs in fundamental chemistry and material science. Development of new algorithms and parallel computing techniques also contribute to LLNL's expertise in numerical simulations, which support the Laboratory's mission in fundamental science and technology.

\section{FY06 Accomplishments and Results}

We (1) extended our first-passage kinetic Monte Carlo (KMC) algorithm from discrete-lattice, 1D diffusion to continuous-space, 3D diffusion using cubes for walker protection; (2) wrote, debugged, and tested a FORTRAN code based on the new algorithm; (3) conducted computational experiments showing that in comparison to the classic method, our new algorithm produces equivalent statistics of particle diffusion and far surpasses the classical algorithm in computational efficiency when the density of diffusing particles is low; and (4) published a paper on the new method.

\section{Proposed Work for FY07}

In FY07, we plan to (1) extend the new method to dealing with diffusion of multiple particles in confined geometries and to mixed 1D/3D diffusion, (2) develop mathematical algorithms for 3D KMC using spherical protective domains to overcome the notorious inefficiency of KMC simulations in cases where large clusters of particles emit monomers and re-absorb them almost immediately, (3) write a parallel KMC code based on a spatial domain decomposition scheme, (4) examine the potential of the new algorithm for boosting performance of quantum Monte Carlo simulations, (5) develop a user-friendly interface for setting up the geometry and creating input decks of unit processes and their kinetic rates, and (6) organize a workshop for potential users from both within and outside the Laboratory.

\section{Publications}

Oppelstrup, T., et al., in press. "First-passage Monte Carlo: diffusion without all the hops." Phys. Rev. Lett. UCRL-JRNL-224951. 
216 LDRD FY2006 Annual Report 
Laboratory Directed Research and Development

\section{Nuclear Science and Engineering}




\title{
Broadband Radiation and Scattering
}

Robert M. Sharpe

\author{
04-ERD-017
}

\begin{abstract}
This project will enhance our computational electromagnetics capability in broadband radiation and scattering analysis, including electromagnetic interference and compatibility, noise analysis, broadband radar, and accelerator wake-field calculations. Current codes are limited by the accuracy of radiation boundary conditions (RBCs), which truncate space. We will develop improved RBCs by extending the perfectly matched layer approach to nonCartesian meshes and by developing discrete-time-domain, boundary-integral techniques, which are compatible with high-accuracy, finite-element methods and capable of arbitrary accuracy. The accuracy and efficiency of the two approaches will be compared for a variety of radiation and scattering problems.
\end{abstract}

The ultimate deliverable is an enhanced computational electromagnetics capability that can provide accurate and efficient computational solutions to broadband radiation and scattering problems. The algorithms for improved RBCs will be incorporated into Lawrence Livermore's existing EMSolve code. The result will be a 10- to 1000-fold improvement in simulation accuracy. Problems that heretofore were not solvable will be addressed. Improved algorithms and our existing high-performance computer hardware will place Lawrence Livermore's computational electromagnetics activity among the top capabilities in the world, thus attracting new talent to the Laboratory.

\section{Mission Relevance}

Our work supports the national security mission by reducing the time and money spent in building and testing finite-element electromagnetics programs, and will enable computer simulations for new devices and systems, performance analysis of systems critical to nonproliferation efforts, and the design of micropower impulse radar and other microwave systems.

\section{FY06 Accomplishments and Results}

Our plan for FY06 has been to develop an enhanced broadband capability for use on LLNL's parallel computers. We (1) developed a fully functional hydrid code (boundary-finite element) that is parallel, stable, and accurate; (2) developed a sub-cycling acceleration method for the boundary-element code segment and demonstrated increases in computational speed with acceptable losses of accuracy; (3) assessed parallel efficiency and solution accuracy; and (4) demonstrated a 1000-fold increase in accuracy over conventional methods that employ absorbing boundary conditions. Though more expensive than conventional methods, the increased accuracy and ability to use much smaller discretizations make our hybrid method attractive for important classes of problems. 


\title{
Publications
}

Champagne, N. J., D. R. Wilton, and J. W. Rockway, 2006. "The analysis of thin wires using higher-order elements and basis functions." Antennas and Propagation Society Intl. Symp. 2006, IEEE, vol. 1, pp. 3887-3890. UCRL-CONF-218970.

Fasenfest, B. J., et al., in press. "Dielectric electrostatic breakdown using boundary element methods." Eng. Anal. Bound. Elem. UCRL-JRNL-214355-DRAFT.

Fasenfest, B., et al., 2006. "A hybrid FEM-BEM unified boundary condition with sub-cycling for electromagnetic radiation." Antennas and Propagation Society Intl. Symp. 2006, IEEE, vol. 1, pp. 2757-2760. UCRL-CONF-218239.

White, D. A., and B. J. Fasenfest, 2006. "Performance of low-rank QR approximation of the finite element Biot-Savart Law." 12th Biennial IEEE Conf. Electromagnetic Field Computation, vol. 1, p. 352. UCRL-CONF-218238.

White, D. A, et al., in press. "A QR accelerated volume-to-surface boundary condition for the finite element solution of eddy current problems." IEEE Trans. Magn.

UCRL-JRNL-224344.

\section{Decontamination of Terrorist-Dispersed Radionuclides from Surfaces in Urban Environments}

Robert P. Fischer

05-ERD-029

\begin{abstract}
We intend to develop radionuclide-specific decontamination agents and optimize them for use in a mass transit infrastructure. The work will address recognized data gaps by advancing the basic scientific knowledge of radionuclide-substrate interactions in urban environments following a terrorist attack with a radiological dispersal device (RDD). The final deliverable will consist of: prototype decontamination agents for americium and cesium released in urban environments, a better understanding of surface decontamination, and a model for radionuclide-surface interactions.
\end{abstract}

This project will identify and optimize a new generation of radionuclide-specific decontamination agents and improved fate-transport modeling for response to an RDD event. This research will result in a substantial improvement over existing response capability, and results will be published in peer-reviewed journals.

\section{Mission Relevance}

This project enhances the U.S. capability to respond effectively to an RDD. It supports national and homeland security and environmental management missions by developing effective decontamination agents for use in urban settings and by advancing the basic scientific knowledge of radionuclide-substrate interactions in the urban environment 


\title{
FY06 Accomplishments and Results
}

In FY06, in an effort to develop urban surface test capabilities, we conducted an RDD contamination experiment at Lawrence Livermore's Contained Firing Facility. The experimental data obtained using laser ablation suggests a faster-than-expected penetration of contaminants into the concrete at depths deeper than expected. We also initiated research into the use of geological fate and transport models to predict the transport of radionuclides in concrete systems. The results indicate that the migration of contaminants into the concrete is being affected by their partitioning in the solid phase. In addition we selected, synthesized, characterized, and investigated four effective decontamination agents for cesium and americium in urban environments, using cryptand and chelator molecules.

\section{Proposed Work for FY07}

In FY07, we will (1) build on our successful FY06 experiment at the Contained Firing Facility by conducting an additional outdoor high-explosive test at Site 300 to examine the effect of wind and unconfined deposition, (2) evaluate test effectiveness of the four chosen decontamination agents on real and surrogate samples exposed to cesium and europium by high-explosive deposition and/or aerosol particle deposition, and (3) identify the attributes of a successful delivery matrix followed by bench-scale laboratory tests in which the chosen decontamination agents have been incorporated into potential delivery matrices such as foam, gel, aerosol, liquid, or a strippable coating.

\section{Publications}

Waen, J. G., R. P. Fischer, and M. Sutton, 2006. Sorption studies of Eu and Cs with urban surfaces. UCRL-POST-223585.

\section{A Coupled, Multiphysics Code for Accurate Modeling of Nuclear Reactors}

\author{
Richard Procassini 06-ERD-069
}

\begin{abstract}
We propose to perform the algorithmic research and development necessary to produce a prototype coupled, multiphysics code for accurate modeling of the core region of nuclear fission reactors. This code, which we call Osiris, will permit reactor designers to overcome many of the limitations they currently face, including use of lumped-parameter models, as well as the use of multiple codes within a multistep modeling methodology. Our approach in developing this new code leverages our proven expertise in code development and our suite of existing code modules. This project will pave the way for Lawrence Livermore to contribute to the future needs of DOE's Global Nuclear Energy Partnership (GNEP) initiative.
\end{abstract}


The goal of our effort is to leverage existing expertise in code development and a suite of existing neutron-transport and thermal-hydraulics code modules to produce a prototype high-performance, coupled, multiphysics code that is applicable to the design of reactor systems. The intent is to replace an existing suite of legacy codes, which require significant approximations and assumptions, with an integrated, coupled code that permits the design of a reactor core using a first-principles approach to model the physics. This approach will greatly simplify the reactor-core design process. The high-performance capabilities of Osiris will enable routine core physics calculations at scales that are impossible for the current modeling methodology.

\section{Mission Relevance}

This project takes advantage of LLNL's expertise in code development and nuclear reactor modeling to provide a tool that will respond to the future needs of the DOE's GNEP initiative, which will contribute to the future of nuclear power options for the United States and help to realize the DOE's goal of enhanced energy security. Additional benefits may be obtained from this work in the area of code validation supporting the Laboratory's Stockpile Stewardship Program.

\section{FY06 Accomplishments and Results}

After a midyear start in July 2006, we investigated and implemented algorithms for delayed-neutron emission in the Amtran deterministic transport code, and investigated algorithms for use in a new depletion and decay module. In addition, our team began to develop the interface code that permits data sharing between thermal-hydraulics and neutral-particle transport modules.

\section{Proposed Work for FY07}

In FY07, we will develop an incompressible Navier-Stokes flow solver from existing code components and combine it with an existing thermal-transport solver. Other areas of algorithm development include parallelization of the Overture thermal-hydraulics module through the use of a parallel, multigrid elliptic equation solver, and extension of the Carter mesh-mapping module to accept the overlapping grids used in Overture. 


\section{LDRD FY06 Annual Report}


Laboratory Directed Research and Development

\section{Physics}




\title{
Laser-Matter Interactions with a 527-Nanometer Drive
}

\author{
Siegfried Glenzer $\quad$ 03-ERD-070
}

\begin{abstract}
The primary goal of this research is to develop an understanding of laser-matter interactions with 527-nm light $(2 \omega)$ for studies of material strength, radiation transport, and hydrodynamics. The potential of significantly greater energy delivered onto targets at $2 \omega$ enables a wide variety of experiments that scale strongly with energy and allow us to probe increasingly higher regimes of pressure, temperature, and strain rate in matter. As part of this project, we are also developing the enabling technology and prototype instrumentation to diagnose a high-fluence laser beam for energy, power, near-field intensity profile, and full-aperture backscatter at $2 \omega$.

As a result of this work, we will resolve important scientific questions relating to laserplasma interactions (backscatter and filamentation), laser-target coupling (absorption) and preheat (hot electrons generated by stimulated Raman scattering), and gain a better understanding of the advantages and limitations of $2 \omega$ illumination relative to $3 \omega$ for highenergy-density (HED) and inertial-confinement fusion (ICF) applications. We will also pave the way for critical stockpile-stewardship-related experiments to be performed in matter in regimes of pressure, temperature, and strain rate much higher than previously accessible.
\end{abstract}

\section{Mission Relevance}

Five key areas within the Stockpile Stewardship Program would significantly benefit from the ability to conduct experiments with a $2 \omega$ drive: ignition, material dynamics, experiments using special nuclear materials, nuclear weapons effects testing, and hydrodynamics. This project will also enable cutting-edge research in ICF, relevant to the Laboratory's stockpile stewardship and energy security missions, and HED physics, in support of stockpile stewardship and the Laboratory's mission in breakthroughs in fundamental science.

\section{FY06 Accomplishments and Results}

We directly measured electron temperature using $4 \omega$ Thomson scattering, validating our two-dimensional radiation-hydrodynamics modeling. We were able to vary the electron temperature from $1.8 \mathrm{keV}$ up to $3.5 \mathrm{keV}$, accessing for the first time an ignition-relevant regime for $2 \omega$ interaction. As predicted by our modeling, transmission through the target reached $80 \%$, while stimulated Raman scattering (SRS) was below $1 \%$ at $3.5 \mathrm{keV}$. In conclusion, this project made major progress in understanding and measuring laser-plasma interaction at $0.527 \mu \mathrm{m}$. An intensity limit (around $3 \times 10^{14} \mathrm{~W} / \mathrm{cm}^{2}$ ) and a minimum electron temperature ( $3 \mathrm{keV}$ ) for efficient coupling of the laser with the target were measured, showing that SRS can be controlled and enabling a $0.527-\mu \mathrm{m}$ option for future HED physics and ICF applications. 


\title{
Characterization and Optimization of High-Energy K-Alpha X-Ray Sources
}

\author{
Hye-Sook Park 03-ERD-072
}

\begin{abstract}
This project will explore the generation, characterization, and optimization of high-energy (20- to $100-\mathrm{keV}) \mathrm{K}$-alpha $\mathrm{x}$-ray backlight sources for application to high-energy petawatt (HEPW) radiography experiments at future large lasers for fusion research. Our goal is to develop the capability to predict and optimize source parameters in HEPW experiments. To this end, we will characterize key K-alpha source parameters as a function of laser and target parameters in experiments at LLNL's Janus-pumped, ultrashort-pulse laser facility, and if possible, at higher-energy, petawatt-scale laser facilities.

By careful characterization of source parameters, we are understanding the fundamental physics responsible for optimization of $\mathrm{x}$-ray yields with respect to these parameters and, by learning more about the physics involved, to be able to optimize backlight targets for specific applications. In particular, we will measure $\mathrm{x}$-ray source sizes and yields from high-atomic-number targets at energies up to $68 \mathrm{keV}$ and determine whether and how laser prepulses affect source characteristics. We will also investigate physically restricted sources, which could enhance source brightness.
\end{abstract}

\section{Mission Relevance}

The use of $x$-ray sources as backlights for radiography experiments is an established technique for studying hydrodynamics, equations of state, and other properties of materials under extreme conditions of high temperature and density. Our project will investigate how to most effectively perform experiments at future large lasers for fusion research by using high-energy $x$-ray backlights to study large-volume, high- $Z$ materials. Success in this effort is directly relevant to the stockpile-assessment activities in support of LLNL's national security mission.

\section{FY06 Accomplishments and Results}

We (1) measured high- $Z$ K-alpha emissions using high-resolution crystal spectroscopy and determined optimal laser condition to maximize K-alpha yield while minimizing the background emission; (2) investigated the effects of physically restricted sources such as small foils and small embedded wires, which were shown to yield enough K-alpha photons for high-energy radiography; (3) explored fundamental issues regarding laser-electron interactions; (4) tested different source size targets and measured their yield differences; and (5) developed new high-energy diagnostics utilizing a multilayer mirror that measures the spectrum of high-energy photons from laser interactions. 


\title{
Publications
}

Notley, M., et al., 2006. "Development of time-resolved K-alpha spectroscopy in highintensity laser-plasma interactions. Rev. Sci. Instrum. 77, 10F322. UCRL-CONF-221180.

\section{Development of Absolute Spectroscopic Diagnostics for Nonlocal-Thermodynamic-Equilibrium Plasmas}

Howard A. Scott $\quad$ 04-ERD-019

\begin{abstract}
The goal of this project is to develop a quantitative understanding of nonlocalthermodynamic-equilibrium (NLTE) plasmas. We are conducting experiments using lowdensity aerogel foams to measure the temporal evolution of the $\mathrm{K}$ - and L-shell emission of highly ionized species. The data will be sufficiently accurate to create benchmarks that can be used to refine and potentially validate Laboratory's NLTE codes, such as CRETIN. In particular, we will use experiments to address the recombination processes in the density regime of $10^{19}$ to $10^{22} \mathrm{~cm}^{-3}$ of mid-atomic-number elements. The experiments will provide temporally resolved, absolute measurements of the emission over a spectral range of 200 to $1000 \mathrm{eV}$. Data will be compared with calculations at each stage of the project.

The data obtained will resolve longstanding discrepancies in the study of laser-produced plasmas and enable better modeling of physical processes relevant to stockpile stewardship. These experiments investigate the NLTE phenomena that impact high-power laser experiments. Deliverables will include (1) absolute spectra from an NLTE plasma, (2) temporal correlation of K- and L-shell spectra, and (3) a new capability to perform absolute emission measurements in the soft x-ray regime, which will provide an important new constraint for simulations.
\end{abstract}

\section{Mission Relevance}

Accurate understanding of the mechanisms of $x$-ray production is an essential component of energy balance and $x$-ray transport in stockpile stewardship applications and for the design and production of high-energy-laser $\mathrm{x}$-ray sources. The physical conditions present in many important stockpile-stewardship applications and virtually all radiation-dominated laboratory experiments are in NLTE plasmas, which is relevant to weapons effects.

\section{FY06 Accomplishments and Results}

In FY06, an additional set of experiments was performed on the NIKE laser to determine plasma temperatures correlated with spectra. A double-sided laser configuration provided improved uniformity, while tamper regions mitigated hydrodynamic expansion effects. Localized dopants provided spectroscopic information on plasma temperatures. Computational advances for the year included both an improved L-shell model along with an upgraded capability for relatively inexpensive NLTE modeling suitable for many applications. 
Data analysis and simulation efforts will continue because of increasing programmatic interest, and the tools and analyses developed under this project have significantly improved our understanding of, and computational capability for, NLTE x-ray spectra.

\title{
Publications
}

Chung, H. K., et al., 2005. "FLYCHK: generalized population kinetics and spectral model for rapid spectroscopic analysis for all elements." High Energ. Dens. Phys. 1, 3.

UCRL-JRNL-213347.

\section{Electronic Transitions and Phonons in f-Band Metals at High Pressures}

Choong-Shik Yoo

04-ERD-020

\begin{abstract}
Unusual phase transitions driven by electron correlation effects occur in many f-band metals. The exact nature of these transitions has not been well understood, including the short-range correlation effects themselves, their relation to long-range crystalline order, the role of magnetic moments and order and the critical behavior, among other issues. Many of these questions represent forefront physics challenges central to stockpile materials. In this study, we propose to investigate electronic phase transitions occurring in solid and liquid f-band metals at high pressures and temperatures using the nation's brightest third-generation synchrotron x-ray source, the Advanced Photon Source (APS). This work will be complemented by new theoretical activities.

The success of the proposed study will result in significant advances of our current understanding in two forefront scientific areas of condensed matter physics: electron correlations and phonons of $\mathrm{f}$-band metals. The study will deliver fundamental data to challenge and validate theoretical understanding of $\mathrm{f}$-electron calculations and interatomic forces and will also develop and apply cutting-edge experimental and theoretical tools.
\end{abstract}

\section{Mission Relevance}

This LDRD should result in better understanding of forefront physics challenges central to stockpile actinides such as electron-correlation effects, phonons, and liquid equations of state for stockpile stewardship. These studies on strongly correlated systems additionally provide opportunities to develop collaborations with leading scientists in the field and attract talented young physicists to the Laboratory.

\section{FY06 Accomplishments and Results}

In FY06 we (1) determined the local magnetic moments of f-electrons in light rare-earth metals (praseodymium, neodymium, and europium) across the volume-collapse transitions 
using high-resolution $\mathrm{x}$-ray emission spectroscopy and (2) determined the mixed-valence electronic states of praseodymium and dysprosium up to $100 \mathrm{GPa}$ using resonant inelastic $\mathrm{x}$-ray scattering. These results provided a systematic understanding of volume collapse transitions in $\mathrm{f}$-metals.

\title{
Publications
}

Kasinathan, D., et al., 2006. "Pressure-induced Kohn anomaly surfaces and superconductivity in compressed lithium." Phys. Rev. Lett. 96, 47004. UCRL-JRNL-226536.

Lazicki, A. K., et al., 2005. "New cubic phase of lithium nitride to $200 \mathrm{GPa}$ : high ionic stability of N3- ions." Phys. Rev. Lett. 95, 165503. UCRL-JRNL-214038.

Lazicki, A. K., et al., 2006. "Pressure-induced antifluorite-to-anticotunnite phase transition in lithium oxide." Phys. Rev. B. 73(18), 184120. UCRL-JRNL-220848.

Maddox, B., et al., 2006. "High-pressure structure of half-metallic $\mathrm{CrO}_{2}$." Phys. Rev. B. 73, 144111. UCRL-JRNL-215214.

Maddox, B., et al., 2006. "Kondo-like $4 f$ delocalization in Gd at high pressures." Phys. Rev. Lett. 96, 215701. UCRL-JRNL-217382.

Yoo, C. S., et al., 2005. "First-order isostructural Mott transition in MnO." Phys. Rev. Lett. 94, 115502. UCRL-JRNL-204811.

\section{Short-Pulse Laser Absorption and Energy Partition at Relativistic Laser Intensities}

Ronnie Shepherd 04-ERD-023

\begin{abstract}
We will conduct detailed and integrated experiments for the first comprehensive measurements of laser absorption and energy partitioning in solid targets heated with an ultrashort laser pulse focused to relativistic laser intensities $\left(>10^{17} \mathrm{~W} / \mathrm{cm}^{2}\right)$. Measurements will include determining the density scale length, which is a critical parameter in determining the contribution of absorption processes dominant at low laser intensities. In parallel, we propose to benchmark LSP, a new collisional particle-in-cell (PIC) model that is planned as the primary modeling tool for future petawatt and high-energy-laser experiments. The resulting information will be used to produce high-brightness, shortpulse-laser broadband $\mathrm{x}$-ray sources for these experiments.
\end{abstract}

We expect to (1) measure scale length versus laser intensity, scale length versus absorption, relative hot electron and thermal electron production, magnetic field strength 
versus laser intensity, and particle energy distribution versus laser intensity; (2) perform energy-scaling absorption experiments; and (3) interface the measurements to codes to improve the fundamental physics used in high-intensity laser-matter interaction models. If successful, we will develop a predictive capability for experimental design and data analysis on petawatt lasers.

\section{Mission Relevance}

This work will provide insight in an area of relevance to weapons physics (i.e., proton generation and $\mathrm{x}$-ray back illuminators) and experiments at future large fusion-class lasers (i.e., hot electrons for fast-ignitor schemes) by acquiring high-quality physics data. This research will also help recruit scientists in high-energy-density physics, in support of stockpile stewardship.

\section{FY06 Accomplishments and Results}

In FY06, we repeated our p-polarization laser absorption measurements and extended the measurements to s-polarized light as well. We compared measurements with our previous results in $\mathrm{p}$ - and s-polarizations. We also measured the angular dependence of nonthermal electrons emitted during the interaction process. These data provide a signature for the absorption mechanism as the focused laser intensity increases from the nonrelativistic to the relativistic regime. Overall, this project has yielded several firsts-we made the first absorption measurements for laser-matter interactions in the relativistic regime. In addition, we performed the first study of partitioning between thermal and nonthermal electrons. These studies will act as a benchmark for the laser-matter interaction community.

\section{Publications}

Kemp, A., et al., in press. "Collisional relaxation of super thermal electrons generated by relativistic laser pulses in dense plasma." Phys. Rev. Lett. UCRL-PRES-225465.

Kemp, A., et al., 2005. Modeling of ultra-fast ionization dynamics in intense short pulse laser-solid interactions. Inertial Fusion Sciences and Applications 2005, Biarritz, France, Sept. 4-9, 2005. UCRL-CONF-216169.

Kemp, A., et al., 2006. Modeling ultra-fast ionization dynamics and electron transport with Monte-Carlo collisional PIC simulations. IEEE Intl. Conf. Plasma Science, Traverse City, MI, June 6, 2006. UCRL-PRES-221909.

Ping, Y., et al., 2006. Absorption and energy partition of short laser pulses in the relativistic regime. 36th Anomalous Absorption Conf., Jackson Hole, WY, June 4-9, 2006. UCRL-ABS-221241. 


\title{
The Creation of a Neutron Star Atmosphere
}

\author{
Richard I. Klein \\ 04-ERD-028
}

\begin{abstract}
Extreme conditions of density and temperature that are relevant to stockpile stewardship are similar to those of low-altitude atmospheres of magnetized neutron stars and accretion disks around black holes. This project will assess the feasibility for experiments on future petawatt lasers to reproduce aspects of these astrophysical environments and assess the possibility of multidimensional codes to experimentally produce and detect photon-bubble instabilities observed by $x$-ray satellites and predicted by LLNL. We will determine the scaling from the astrophysical to the laboratory environment and test key components of the design on existing lasers; perform simulations to estimate temperatures, radiation fields, and magnetic fields; and perform experiments on existing ultraintense lasers.

Assessing the feasibility of a scaled future experimental design to reproduce aspects of magnetized neutron star atmospheres and radiation-dominated accretion disks around black holes will advance the study of extreme physics generated by strongly radiative flows and laser-plasma interactions for the laboratory study of both distinct astrophysical phenomena and the physics of extreme conditions relevant to stockpile stewardship. Establishing a laboratory capability to probe the physics of accreting, magnetized compact objects and accretion disks will enhance LLNL's international reputation in high-energydensity (HED) physics.
\end{abstract}

\section{Mission Relevance}

This project has direct relevance to understanding the extreme conditions of HED, ultrashort-pulse-laser-matter interactions that are relevant to LLNL's stockpile stewardship mission and will be a driver for advanced scientific applications at future petawatt lasers where Laboratory personnel will carry out their work. The world-class science that this project represents will improve recruiting and forge links with the U.S. astrophysical community.

\section{FY06 Accomplishments and Results}

In FY06, we (1) completed the atomic physics analysis of our data-using the particle-incell three-dimensional electromagnetic code LSP (Laser Shock Processing) -to establish the extreme temperatures possible in petawatt laser-solid interactions; (2) conducted experiments with proton deflectometry and optical Faraday rotation using the code Titan to measure the B fields of such interactions; (3) analytically established the scaling parameters from the astrophysical to the laboratory petawatt environment and the phase space of those parameters that give rise to the astrophysical phenomena; (4) assessed whether existing laboratory platforms can achieve scaled conditions required to duplicate the astrophysical phenomena; and (5) assessed whether actual petawatt lasers can achieve such conditions. We concluded that such conditions will be achievable with highgain ignition on future fusion-class lasers. 


\title{
Publications
}

Gregori, G., et al., 2004. "Experimental characterization of a strongly coupled solid density plasma generated in a short-pulse laser target interaction." Contrib. Plasma Phys. 45, 284-292. UCRL-JRNL-211077.

Moon, S. J., et al., 2005. "A neutron star atmosphere in the laboratory with petawatt lasers." Astrophys. Space Sci. 298, 293-298. UCRL-JRNL-211863.

\section{New Generation X-Ray Optics: Focusing Hard X Rays}

Regina Soufli 04-ERD-032

\begin{abstract}
This research aims to develop a new generation of optics based on hard $x$-ray (up to $200 \mathrm{keV}$ ) reflective and diffractive focusing elements with unprecedented angular resolution. These optics, which leverage core capabilities developed at LLNL such as the precise fabrication and mounting of thermally formed substrates and smoothing, have immediate applications in areas including high-energy physics, biomedical research, astrophysics, shot diagnostics at large-scale laser facilities, and experiments at nextgeneration light sources. The final year of this work concentrates on extracting science from the advances of the first two years through collaboration in the CERN Axion Solar Telescope (CAST) experiment.
\end{abstract}

Our research will lead to a novel approach for manufacturing high-quality, low-cost reflective $x$-ray optics. This includes the development of novel substrate materials, innovative techniques to accurately integrate individual mirrors into a complex configuration, and simulation tools to design and model grazing incidence optics. To demonstrate the full potential of this new capability, we will design and construct a 14-shell concentrating optic for the CAST experiment. Simulations indicate that use of our $x$-ray optics at CAST will increase the overall sensitivity in detecting high-mass axions by a factor of two.

\section{Mission Relevance}

The demonstration of this novel technological capability will have immediate impact on $\mathrm{x}$ ray diagnostics for target and plasma characterization at future large fusion-class lasers, in support of the stockpile stewardship mission. The new capability will also find application for basic science missions at other next-generation lasers and light sources (e.g., the Linac Coherent Light Source), in major NASA programs (NuSTAR and Constellation-X), and for medical applications to improve human health.

\section{FY06 Accomplishments and Results}

In FY06, as full collaborators in the CAST dark-matter experiment, we helped guide experimental protocols and data analysis. Most significantly, we fabricated and tested the 
14-shell concentrating optic specifically designed for CAST. In addition to improving the detection sensitivity of CAST, construction of the optic allowed us to exercise all of the techniques and procedures we have developed. Using CAST facilities-including PANTER, a unique long-beam $\mathrm{x}$-ray calibration facility operated by one of this project's collaboratorswe made detailed measurements over a three-week campaign that demonstrated the effectiveness of the next-generation reflective $x$-rays optics developed in this project.

\title{
Publications
}

Abbon, P., et al., 2006. A low background micromegas detector for the CAST experiment. 9th Intl. Conf. Advanced Technology and Particle Physics (ICATPP) Mtg., Como, Italy, Oct. 17-21, 2005. UCRL-CONF-222638.

Bajt, S., et al., 2006. Multilayers in Laue geometry. 8th Physics of X-ray Multilayer Structures Conf., Sapporo, Japan, Mar. 12-16, 2006. UCRL-ABS-217208.

Pivovaroff, M., 2006. Multilayer x-ray optics for biomedical imaging. 8th Physics of X-Ray Multilayer Structures Conf., Sapporo, Japan, Mar. 12-16, 2006. UCRL-ABS-218261.

\section{Nanomechanics: Strength and Structure for Nanotechnology}

Robert E. Rudd

$$
\text { 04-ERD-043 }
$$

\begin{abstract}
We are developing the fundamental principles of nanomechanics, which concerns the mechanical behavior of nanoscale structures, by advancing the theoretical understanding of novel mechanical phenomena at the nanoscale and by developing computational methodologies suitable for nanomechanical systems. We will focus on mechanical nanoscale processes driven by surface effects, in particular, how the stiffness and strength of nanostructures are affected by their relatively large surfaces, and how these effects can be used to control self-assembly. We are investigating two kinds of systems: (1) nanoelectromechanical systems (NEMS) to study size-dependent stiffness and strength effects and (2) epitaxial nanostructures, which exemplify nanomechanical self-assembly.

We expect to develop a new theory of stiffness and strength of nanostructures and a deeper understanding of how structures are produced in nanomechanically driven selfassembly. We will conduct atomistic calculations of the properties of nanostructures using both classical interatomic force laws and ab initio quantum mechanical calculations, as well as conduct simulations of the plastic deformation of nanoscale pillars. This work has extensive implications for the mechanical response of nanoscale structures in NEMS, foams, and nanotechnology and biotechnology.
\end{abstract}




\section{Mission Relevance}

The scientific understanding and computational tools developed have potential application to the control of interface features in fusion-class laser targets, the development of nextgeneration biothreat detectors for homeland security applications, and the mechanical characterization of protein-ligand binding of interest to biological science. Applications associated with dislocation nucleation are relevant to the Advanced Simulation and Computing Program, which supports stockpile stewardship. In addition, the fundamental principles of nanomechanics discovered in this project will contribute to the Laboratory's basic science mission.

\section{FY06 Accomplishments and Results}

In FY06, we (1) simulated dynamical dissipative processes in silicon-NEMS flexural nanoresonators using molecular dynamics and the hybrid finite element/molecular dynamics code FEMD, (2) simulated the plastic deformation of metallic nanowires, (3) completed the first ever ab initio quantum calculations of nanowire mechanics, and (4) conducted the first discrete dislocation-dynamics simulation of the deformation of metallic nanopillars. Overall, we have made significant advances in nanomechanical elasticity, plasticity, and self-assembly. We have developed new computational tools including hybrid atomistic and finite element modeling of nanostructures and dislocation dynamics methodologies for systems with surfaces. We published numerous journal articles and gave several invited talks. Our leadership in this field has been recognized by an invitation to organize a special journal issue on nanomechanics.

\section{Publications}

Lee, B., in press. "First-principles study of the Young's modulus of $\mathrm{Si}(001)$ nanowires." Phys. Rev. Lett. UCRL-JRNL-222598.

Lee, B., and R. E. Rudd, in press. "First-principles calculation of mechanical properties of $\mathrm{Si}(001)$ nanowires and comparison to nanomechanical theory." Phys. Rev. B.

UCRL-JRNL-225464.

Lee, B., and R. E. Rudd, 2006. First principles calculation of the Young's modulus of silicon (001) nanowires. Fall Mtg. Materials Research Society, Boston MA, Nov. 27-Dec. 1, 2006. UCRL-ABS-222344.

Rao, S. I., in press. "Large scale 3-dimensional dislocation simulations reveal mechanisms for strengthening at micrometer scale." Nature. UCRL-JRNL-222934.

Rudd, R. E., 2006. Nanomechanics: concurrent multiscale modeling of mechanical components of NEMS. GE Workshop on Computational Techniques for Nanoscale Physics, Niskayuna, NY, Jan. 16-18, 2006. UCRL-PRES-218082.

Rudd, R. E., and J. Q. Broughton, 2006. "Coarse-grained molecular dynamics: nonlinear finite elements and finite temperature." Phys. Rev. B. 72, 144104. UCRL-JRNL-212644.

Rudd, R. E., D. R. Mason, and A. P. Sutton, in press. "Lanczos and recursion techniques for multiscale kinetic Monte Carlo simulations." Prog. Mater. Sci. UCRL-JRNL-219802. 
Rudd, R. E., et al., in press. "Equilibrium distributions and the nanostructure diagram for epitaxial quantum dots." J. Comput. Theor. Nanosci. UCRL-JRNL-221029.

Tang, M., et al., 2006. "A hybrid method for computing forces on curved dislocations intersecting free surfaces in three-dimensional dislocation dynamics." Model. Simul. Mater. Sci. Eng. 14, 1139. UCRL-JRNL-212816.

Tang, M., 2006. Discrete dislocation dynamics simulations in finite systems using ParaDiS. Plasticity 2006, Halifax, Nova Scotia, Canada, July 17-22, 2006. UCRL-ABS-217932.

Tang, M., G. Xu, and G. Hommes, 2006. Discrete dislocation dynamics simulation of plasticity in small systems using ParaDiS. Fall Mtg. Materials Research Society, Boston, MA, Nov. 27-Dec. 1, 2006. UCRL-ABS-222250.

\title{
High-Average-Power, High-Energy, Short-Pulse Fiber Laser System
}

\author{
Michael J. Messerly 04-ERD-048
}

\begin{abstract}
Our objective is to develop the technology for high-energy ( $>10 \mathrm{~mJ})$, high-averagepower $(>300 \mathrm{~W}$ ) fiber laser systems. The primary limits to scaling the output energy in a fiber laser are nonlinear interactions and beam quality for larger core sizes. To increase the output energy of a fiber amplifier, we are developing a novel waveguide design that distributes the optical intensity of the light more evenly across the core. Consequently, smaller cores can produce pulses with higher output peak powers.

We will develop the key technology components for a high-energy, high-average-power, short-pulse fiber laser system to scale the pulse energy and average output power of short-pulse fiber laser systems by an order of magnitude beyond previously reported results. We will also demonstrate a prototype system. This should solve many longstanding research hurdles to make the technology suitable for applications in materials processing, high-resolution $\mathrm{x}$-ray imaging, and short-pulse front ends for large-scale, high-energy laser systems. We expect this research to lead to a number of spin-offs.
\end{abstract}

\section{Mission Relevance}

High-average-power, high-energy, short-pulse fiber laser systems that are easy to use, reliable, and efficient are relevant to (1) high-energy petawatt laser front ends for future large laser systems; (2) cutting, drilling, and other materials-processing applications; and (3) small-spot-size, high-flux x-ray sources. All three of these areas support DOE and LLNL's national security mission, specifically in stockpile stewardship. 


\title{
FY06 Accomplishments and Results
}

As a result of our investigations of large, flattened-mode fiber designs, we achieved $1-\mathrm{mJ} /$ pulse, 1 -ns pulses at $10 \mathrm{kHz}$ ( $10 \mathrm{~W}$ average power) that could be focused to initiate air breakdown at a threshold intensity of $<10^{10} \mathrm{~W} / \mathrm{cm}^{2}$. We also investigated a large-modearea polarizing photonic crystal fiber, achieving $300-\mu \mathrm{J}$ pulse energies with good beam quality. Pulses were recompressible to $<2 \mathrm{ps}$, and short-pulse air breakdown occurred at an intensity of $>10^{14} \mathrm{~W} / \mathrm{cm}^{2}$. We also investigated the use of both of our bulk optic stretcher designs for fiber chirped-pulse amplification and chirped-fiber Bragg grating stretchers. This project greatly enhanced the Laboratory's understanding of short-pulse, high-energy, high-average-power fiber laser systems.

\section{Development and Application of a Predictive Computational Tool for Short-Pulse, High-Intensity Target Interactions}

Max Tabak

04-ERD-054

\begin{abstract}
The goal of this theory-and-computation effort is to produce and validate a computational tool that can model a high-density plasma driven by high-intensity laser light. Integral components of this model will be coupling between laser light and a dense plasma, production and transport of relativistic electrons, self-consistent production and transport of large-scale electric and magnetic fields, realistic equations of state (EOS), electrical and thermal transport properties of thermal plasmas, and coupling to a hydrodynamic (burn) code. This tool will be used to model past and ongoing experiments driven by highintensity lasers to provide optimized designs for applications such as fast ignition.
\end{abstract}

If the project is successful, we will be able to model experiments driven with ultraintense lasers, from the initial laser-plasma interaction that generate energetic electrons through to the heating of the dense plasma fuel by these particles. This will enable the design and optimization of various applications in high-energy-density physics and fusion energy. We will also design experiments for future large laser systems to obtain EOS and opacity data of relevance to stockpile stewardship; improve the efficiency of radiography techniques; and improve fast-ignition target designs, reducing the short-pulse laser energy necessary for ignition.

\section{Mission Relevance}

Powerful short-pulse, high-intensity lasers are expected to enable a number of applications that are important to defense and fusion energy missions that are difficult, if not impossible, to accomplish by other means. By providing theory and computational support for present and future integrated high-intensity, short-pulse laser efforts, including laser construction and experiment, this project supports LLNL's missions in national and energy security. 


\section{FY06 Accomplishments and Results}

In FY06, we performed the first integrated fast ignition calculation for OMEGA EP laser experiments. For these calculations, we used the code LASNEX to model hydrodynamic evolution and to create profiles for use in modeling the production and transport of hot electrons with the three-dimensional electromagnetic code LSP. These initial studies showed poor energy coupling of the high-intensity laser light to the dense fuel core. Therefore, we studied the shape and size of the cone to maximize the short-pulse laser absorption. We used the hydrocode-linked LSP to model plausible Omega EP designs and found that at plausible laser parameters these designs would fail. In response, we reconsidered target details and found implosion designs with twice the efficiency of previously published designs. We also installed transport algorithms to improve the energy conservation of scattering in LSP, noted other failings in the LSP scattering algorithm, and devised a more accurate algorithm replacement. Overall, in this project we developed a computational tool to model high-density plasma driven by high-intensity laser light. Our improvements to the LSP code have been adopted for incorporation into future releases, and studies using our code to optimize fast-ignition designs have begun.

\section{Publications}

Atzeni, S., and M. Tabak, 2006. "Overview of ignition conditions and gain curves for the fast ignitor." Plasma Phys. Control. Fusion 47, B4769. UCRL-JRNL-213654.

Clark, D. S., and M. Tabak, 2005. Self-similar isochoric implosions for fast ignition. APS Division of Plasma Physics, Denver, CO, Oct. 24-28, 2005. UCRL-POST-216158.

Clark, D. S., and M. Tabak, 2006. Isochoric implosions for fast ignition. APS Division of Plasma Physics, Philadelphia, PA, Oct. 30-Nov. 3, 2006. UCRL-ABS-222867.

Clark, D. S., and M. Tabak, 2006. Isochoric implosions for fast ignition. 29th ECLIM Mtg., Madrid, Spain, June 11-16, 2006. UCRL-PRES-221782.

Cottrill, L. A., et al., 2005. Simulations of relativistic electron beam instabilities for applications to fast ignition. APS Division of Plasma Physics, Denver, CO, Oct. 24-28, 2005. UCRL-POST-216238.

Lasinski, B. F., et al., 2006. PIC simulations of short-pulse, high-intensity light impinging on structured targets. APS Division of Plasma Physics, Philadelphia, PA, Oct. 30-Nov. 3, 2006. UCRL-ABS-213202.

Lasinski, B. F., et al., 2006. Using explicit PIC results in transport codes. Fast Ignition Workshop, Cambridge, MA, Nov. 3-5, 2006. UCRL-ABS-213202.

$\mathrm{Li}, \mathrm{C}$. K., et al., 2006. "Measuring $\mathrm{E}$ and $\mathrm{B}$ fields in laser-produced plasmas with monoenergetic protons radiography." Phys. Rev. Lett. 97, 135003. UCRL-JRNL-220964.

$\mathrm{Li}, \mathrm{C} . \mathrm{K}$., et al., in press. "A monoenergetic proton backlighter for measuring $\mathrm{E}$ and $\mathrm{B}$ fields and for radiographing implosions and HED plasmas." Rev. Sci. Instrum.

UCRL-JRNL-221036. 
Logan, B. G., et al., 2006. "Assessment of potential for ion driven fast ignition." Fusion Sci. Tech. 49, 399. UCRL-JRNL-208443.

Tabak, M., 2005. Capsule optimization techniques for fast ignition. APS Division of Plasma Physics, Denver, CO, Oct. 24-28, 2005. UCRL-ABS-210652.

Tabak, M., 2006. Issues in fast ignition. Anomalous Absorption Conf., Jackson Hole, WY, June 5, 2006. UCRL-PRES-221820.

Tabak, M., in press. "On the path to fusion energy." European Journal of Physics D. UCRL-JRNL-217699.

Tabak, M., and D. Clark, 2006. Issues in assembling fuel for fast ignition. APS Division of Plasma Physics, Philadelphia, PA, Oct. 30-Nov. 3, 2006. UCRL-ABS-222990.

Tabak, M., et al., 2006. "Fast ignition: overview and background." Fusion Sci. Tech. 49, 254. UCRL-JRNL-213396.

Town, R. P. J., et al., 2006. Integrated modeling of fast ignition experiments. Fast Ignition Workshop, Cambridge, MA, Nov. 3-5, 2006. UCRL-ABS-222479.

Town, R. P. J., et al., 2006. Integrated modeling of short-pulse laser-plasma experiments. APS Division of Plasma Physics, Denver, CO, Oct. 24-28, 2005. UCRL-ABS-210615.

Town, R. P. J., et al., 2006. Proton deflectometry of electric and magnetic fields. APS Division of Plasma Physics, Philadelphia, PA, Oct. 30-Nov. 3, 2006. UCRL-ABS-222480.

\title{
Surrogate Nuclear Reactions and the Origin of Heavy Elements
}

Jutta E. Escher $\quad$ 04-ERD-057

\begin{abstract}
This work explores an innovative method for indirectly determining nuclear-reaction cross sections by "surrogate" reactions. This is important because a large number of nuclear reactions relevant to astrophysics and Lawrence Livermore programs cannot be measured with currently available techniques. We will provide the theoretical and experimental framework for planning and analyzing experiments that will allow us to obtain the cross sections of interest. We intend to design, carry out, and analyze experiments that provide benchmarks for the surrogate nuclear reaction method. Our applications will focus particularly on reactions involving unstable nuclei that play a key role in the production of the elements between iron and uranium.

The long-term goal of this research is to establish a new method for indirectly determining cross sections for a large class of nuclear reactions. Indirect approaches are becoming increasingly important as nuclear physics applications, particularly in the area of astrophysics, require information on reactions involving unstable isotopes. While currently available indirect methods focus on direct reactions (i.e., transfer, or stripping or pickup reactions), the surrogate nuclear reaction approach allows us to address the need for
\end{abstract}


compound nuclear-reaction cross sections. Such cross sections are extremely important for achieving an improved understanding of the astrophysical s-process (slow neutron capture) and for obtaining significant new insights into the synthesis of heavy elements.

\section{Mission Relevance}

Establishing a novel technique for determining reaction cross sections on unstable nuclei will satisfy critical needs for science-based stockpile stewardship and homeland security applications that require reliable information about reactions involving these nuclei. The basic science research in this project supports the Laboratory's mission in breakthrough science and technology.

\section{FY06 Accomplishments and Results}

In FY06, we (1) developed the formalism to include collectivity in our description of inelastic scattering; (2) studied pickup reactions; (3) expanded models to treat deformed nuclei; (4) carried out and analyzed a surrogate experiment that allows us to extract the gadolinium-155 ( $n$, gamma) cross section and establish a benchmark for applying the surrogate method to deformed nuclei, as well as studied approximations to the method; and (5) presented our results in journal articles, reports, and at conferences. Overall, we developed tools and expertise for planning and carrying out surrogate experiments and interpreting the results. We greatly enhanced the interest of the science community in the surrogate method, and formed collaborations with universities and other national labs.

\section{Publications}

Bernstein, B. J., et al., 2006. "Deducing the ${ }^{237} \mathrm{U}(\mathrm{n}, \mathrm{f})$ cross section using the surrogate ratio method." Phys. Rev. C. 73, 054604. UCRL-JRNL-217959.

Dietrich, F. S., 2006. Expressions for form factors for inelastic scattering and charge exchange in plane-wave, distorted-wave, and coupled-channels reaction formalisms. UCRL-TR-224742.

Dietrich, F. S., 2006. Surrogate nuclear reactions-an indirect approach for obtaining nuclear reaction data. 9th Intl. Conf. Nucleus Nucleus Collisions, Rio de Janeiro, Brazil, Aug. 28-Sept. 1, 2006. UCRL-ABS-220697.

Escher, J., 2006. Compound-nuclear reaction cross sections via surrogate reactions. 11th Intl. Conf. Nuclear Reaction Mechanisms, Varenna, Italy, June 12-16, 2006. UCRL-PRES-222548.

Escher, J., 2006. Compound-nuclear reaction cross sections via the surrogate method: considering the underlying assumptions. 2006 Ann. Mtg. Division of Nuclear Physics of the American Physical Society, Nashville, TN, Oct. 25-28, 2006. UCRL-ABS-222547.

Escher, J., 2006. Surrogate nuclear reaction methods for astrophysics and other applications. 19th Intl. Conf. Application of Accelerators in Research and Industry, Fort Worth, TX, Aug. 20-25, 2006. UCRL-PRES-224112. 
Escher, J., 2006. Surrogate reactions for advanced fuel cycles. Nuclear Physics and Related Computational Science R\&D for Advanced Fuel Cycles Workshop, Bethesda, MD, Aug. 10-12, 2006. UCRL-PRES-223747.

Escher, J., and F. S. Dietrich, 2006. "Determining compound-nuclear reaction cross sections via surrogate reactions: approximation schemes for $(n, f)$ reactions." Proc. 11th Intl. Conf. Nuclear Reaction Mechanisms, 2006. UCRL-PROC-222939.

Escher, J. E., and F. S. Dietrich, in press. "Determining $(n, f)$ cross sections for actinide nuclei indirectly: examination of the surrogate ratio method." Phys. Rev. C.

UCRL-JRNL-221555.

Escher, J., F. S. Dietrich, and C. Forssen, in press. "Surrogate nuclear reaction methods for astrophysics." Nucl. Instr. Meth. B. UCRL-JRNL-224284.

Forssen, C., et al., 2006. Compound-nuclear reaction cross sections via surrogate measurements. Intl. Symp. Nuclear Astrophysics-Nuclei in the Cosmos IX, Geneva, Switzerland, June 25-30, 2006. UCRL-PROC-223820.

\title{
Stellar Astrophysics and a Fundamental Description of Thermonuclear Reactions
}

\author{
William E. Ormand $\quad$ 04-ERD-058
}

\begin{abstract}
This project is investigating new methods to provide a comprehensive understanding of reactions between light nuclei in hot, dense environments, such as stellar interiors and implosion capsules for future large fusion-class lasers. The project will develop a new theoretical framework that describes the dynamics of nuclear collisions based on the fundamental interactions of nature. In addition, the project will undertake a theoretical study of the quantum corrections to electron screening in thermal plasmas to resolve a discrepancy exhibited in previous theoretical approaches and investigate the viability of using fusion-laser facilities to observe a wide range of stellar thermonuclear reactions.

Achieving an accurate and predictive theoretical view of thermonuclear reactions in thermal plasmas based on fundamental physical properties will have far-reaching applications for astrophysics. Benefits include improved stellar modeling capabilities that could improve our knowledge of neutrino oscillations, better databases important to stockpile stewardship, and higher confidence in understanding the dynamics of fusionclass-laser target capsules. The ability to perform stellar astrophysics experiments at such facilities could lead to an exciting new experimental program.
\end{abstract}




\section{Mission Relevance}

A more comprehensive understanding of nuclear reactions in hot, dense systems based on fundamental physical properties will be achieved. In particular, this project will support the nuclear data effort for the Laboratory's stockpile stewardship mission and provide insight for experiments to be performed at future fusion-class laser facilities. In addition, by advancing the understanding of stellar evolution, this project supports LLNL's basic science mission.

\section{FY06 Accomplishments and Results}

We continued development of the reaction theory formalism by merging the resonating group method (RGM) and the no-core shell model. We derived the formalism for clusteroverlaps, the folding potential, and the norm kernel and developed computer programs to evaluate overlaps for up to four nucleon clusters and the norm kernel for the RGM. We applied the first-generation of our formalism to ${ }^{7} \mathrm{Be}(\mathrm{p}, \gamma)^{8} \mathrm{Be},{ }^{3} \mathrm{He}\left({ }^{4} \mathrm{He}, \gamma\right){ }^{7} \mathrm{Be},{ }^{3} \mathrm{H}\left({ }^{4} \mathrm{He}, \gamma\right){ }^{7} \mathrm{Li}$, and ${ }^{11} \mathrm{Be}(\mathrm{n}, \gamma)^{12} \mathrm{Be}$, and in combination with the potential model, performed calculated $\mathrm{n}+{ }^{4} \mathrm{He}$ scattering and astrophysical S-factors. We also determined the form of the threenucleon force using effective-field theory, and examined the sensitivity of the overlap functions to the three-nucleon force. Overall, the project extended ab initio structure capabilities to reactions, and permitted LLNL to join a Scientific Discovery through Advanced Computing Program collaboration.

\section{Publications}

Barrett, B. R., et al., 2006. "From non-hermitian effective operators to large-scale no-core shell model calculations for light nuclei." J. Phys. A-Math. Gen. 39, 9983.

UCRL-PROC-219600.

Caurier, E., and P. Navratil, 2006. "Proton radii of ${ }^{4} \mathrm{He},{ }^{6} \mathrm{He}$, and ${ }^{8} \mathrm{He}$ isotopes from highprecision nucleon-nucleon interactions." Phys. Rev. C. 73, 021302. UCRL-JRNL-217508.

Navratil, P., C. A. Bertulani, and E. Caurier, 2006. "7Be $(\mathrm{p}, \gamma)^{8} \mathrm{~B}$ S-factor from ab initio wave functions." Phys. Lett. B. 634, 191. UCRL-JRNL-214705.

Navratil, P., C. A. Bertulani, and E. Caurier, 2006. "7Be( $(\mathrm{p}, \gamma)^{8} \mathrm{~B}$ S-factor from ab initio nocore shell model wave functions." Phys. Rev. C. 73, 065801. UCRL-JRNL-217766.

Negret, A., et al., 2006. "Gamow-Teller strengths in the $A=14$ multiplet: a challenge to the shell model." Phys. Rev. Lett. 97, 062502. UCRL-JRNL-223644.

Nogga, A., et al., 2006. "Spectra and binding energy predictions of chiral interactions for 7Li." Phys. Rev. C. 73, 064002. UCRL-JRNL-217514.

Stetcu, I., et al., 2006. "Long- and short-range correlations in the ab-initio no-core shell model." Phys. Rev. C. 73, 037307. UCRL-JRNL-218373. 


\title{
High-Energy-Density Galaxy Jets
}

\author{
Willem Van Breugel 04-ERD-059
}

\begin{abstract}
Jets, ionizing radiation, and high-energy particles from active supermassive black holes and exploding stars profoundly affect the properties of the interstellar medium in their parent galaxies, and thereby the formation of stars. At Lawrence Livermore, we are in a unique position to investigate this by combining our expertise in complex, multidimensional numerical simulations and high-energy experiments with our access to world-class observatories. We propose to complete our multidisciplinary studies, using heavy ion accelerators, of the interaction of jets with interstellar medium clouds and the energetic processing of astrophysically relevant materials.
\end{abstract}

We expect to show that high-energy irradiation experiments and numerical simulations can explain how crystalline interstellar dust in our galaxy is transformed to amorphous dust by cosmic rays, a long-standing puzzle. We hope to add an important, high-energy component to research on materials in extreme, astrophysical conditions that is of use for studies of feedback by active black holes and exploding stars in forming galaxies. Finally, we will have forged successful collaborations in new directions with several universities. Such collaborations advance the code development of high-energy-density jets and radiation and contribute to studies of the effects of particle irradiation of carbon- and silica-related materials.

\section{Mission Relevance}

Our research will help validate computer codes used to simulate high-energy-physics phenomena critical to nuclear physics research and to experiments at future large lasers for fusion studies. Our laboratory experiments, combined with astronomical observations, will provide new information about the effects of high-energy particles on materials under extreme conditions, which will further the national security mission in the stockpile program. Furthermore, the interplay of experiments and modeling of irradiated minerals leading to changes in their material properties also supports forensic capability relevant to nonproliferation.

\section{FY06 Accomplishments and Results}

In FY06, we completed (1) analysis of a dramatic jet-cloud collision, (2) experiments of $10-\mathrm{MeV}$ heavy-ion irradiation of forsterite at cosmic fluences, and (3) simulations of numerical molecular dynamics, which showed that the amorphization can be explained using a thermal spike model. It validates our numerical simulation approach and opens up tremendous possibilities of understanding astrophysical processes of materials at the atomic scale. We also completed 0.5-GeV iron-ion experiments at the Lawrence Berkeley National Laboratory 88-inch cyclotron using forsterite as a target at cosmically relevant fluences. We discovered stoichiometric changes in the surface layers and partial amorphization along discontinuous tracks, providing new diagnostics of astromaterials in energetic environments. 


\title{
Publications
}

Bringa, E. M., in press. "Energetic processing of interstellar silicate grains by cosmic rays." Astrophys. J. UCRL-JRNL-221329.

Croft, S., et al., 2006. "Minkowski's object: a starburst triggered by a radio jet, revisited." Astrophys. J., 647, 1040. UCRL-JRNL-219648.

Dopita, M. A., et al., 2006. "Modeling the pan-spectral energy distribution of starburst galaxies: II. Control of the HII region parameters." Astrophys. J., 647, 244.

UCRL-JRNL-220864.

Durham, P. R., et al., in press. "Atomistic simulation of cosmic ray-induced amorphization of silicate grains." Astrophys. J. Lett. UCRL-JRNL-221330.

Laag, E. A., et al., 2006. "Adaptive optics imaging survey of luminous infrared galaxies."

Astrophys. J., 131, 2877. UCRL-JRNL-220895.

\section{High-Brightness, Laser-Driven, X-Ray Source for Nanoscale Metrology and Femtosecond Dynamics}

\author{
Craig Siders $\quad$ 04-ERD-064
}

\begin{abstract}
This project will develop a new, bright, ultrafast $x$-ray source based on laser-driven $\mathrm{K}$-alpha generation that will produce an x-ray flux 10 to 100 times greater than current microfocus $x$-ray tubes. The short-pulse $(\sim 100 \mathrm{fs})$ duration of this $x$-ray source also makes it ideal for observing time-resolved dynamics of atomic motion in solids and thin films. We are currently developing this K-alpha $\mathrm{x}$-ray source using the existing Falcon terawatt laser system, measuring x-ray yield, emitter size, and temporal pulse length as a function of laser pulse and target characteristics. The measurements are compared with a model. The results will lead to a high-average-power fiber-laser-driven $\mathrm{x}$-ray source.
\end{abstract}

We expect to develop a high-average-brightness $\mathrm{x}$-ray source with subpicosecond temporal and submicron spatial resolution. This source could be used for the in situ radiography of beryllium shells and cryotargets, dynamic diffraction experiments with actinides, and microscopic imaging of chemical and biological systems, nanostructures, and advanced semiconductor devices. Careful measurements of K-alpha x-ray generation versus laser and target parameters will help verify codes being developed for high-energy-density physics and backlighters. When combined with high-average-power, ultrafast fiber-laser technology, this $x$-ray source could serve as a multiple-application facility.

\section{Mission Relevance}

An improved, high-average-power $\mathrm{x}$-ray source will be useful to many applications that support the national security mission, such as the in situ characterization of cryotargets for 
fusion ignition, three-dimensional imaging of actinides under dynamic stress (when used with ultrafast electron diffraction technology), advanced streak cameras, $x$-ray detectors, and $\mathrm{x}$-ray optics.

\title{
FY06 Accomplishments and Results
}

In FY06, we completed the K-alpha source measurements on the Falcon laser. We designed and implemented a bulk optic pulse stretcher and matching pulse compressor on a fiber laser system. Initial $x$-ray source experiments were performed using our femtosecond $\mathrm{x}$-ray source chamber. A small focus $(1.2 \times 1.9 \mu \mathrm{m})$, a pulse energy of a few hundred microjoules, and a pulse duration of a few hundred femtoseconds were independently measured. Although peak intensities sufficient for femtosecond air ionization were achieved, peak intensity was not sufficient for $\mathrm{x}$-ray generation due to incomplete recompressibility of the pulse. The project's final report will describe the path toward a high-brightness, multiple-application x-ray facility.

\section{Ultrafast, in situ Probing of Shocked Solids at the Mesoscale and Beyond: A New Paradigm for Materials Dynamics}

\author{
Hector E. Lorenzana 04-ERD-071
}

\begin{abstract}
For this project, we will conduct the first unambiguous measurements of lattice and microstructural evolution in metals under high loading rates using ultrafast x-ray and electron probes. Novel, dynamic experimental techniques will be developed for electron microscopes, laser-based $\mathrm{x}$-ray sources, and accelerator-based x-ray facilities. We will use diffraction, scattering, and imaging measurements to observe the microscopic processes governing response of the material in situ at the nanometer scale with nanosecond resolution. These measurements will be linked to the underlying physics that ultimately determine material response.
\end{abstract}

We expect to carry out measurements at the microscopic level that will provide insight into the basic processes that govern solid-state material response and determine the evolution of material properties. Research will focus on the ability to measure shock-driven phase transitions in materials on the ultrafast (nanosecond) time scale. With the emergence of short-pulse laser-based and accelerator-based x-ray sources, the ability to make these measurements at the lattice level in real time will position Lawrence Livermore as a world leader in the growing field of ultrafast materials science.

\section{Mission Relevance}

Success in stockpile stewardship, high-energy-density science, and other national security mission areas demand a fundamental understanding of dynamic materials behavior. The expertise developed in this project will further current and future research in support of stockpile stewardship at facilities such as the Joint Actinide Shock Physics Experimental 
Research (JASPER) gas gun at the Nevada Test Site and basic science facilities such as the Linac Coherent Light Source at Stanford. This work will drive development of computational tools for predicting performance, safety, and reliability of nuclear weapons, as well as for high-power laser research.

\section{FY06 Accomplishments and Results}

In FY06, we continued our analysis of the solid-solid phase change observed in iron using in-situ $x$-ray diffraction, focusing on the kinetics of transformation as well as the rearrangement path of the atoms. Experiments on titanium, combining in-situ x-ray diffraction and Velocity Interferometer System for Any Reflector (VISAR), suggest a solid-solid phase transition, which occurs at a higher pressure than measured in static experiments. This project has been successful in making lattice response measurements in shocked materials. One of the major highlights was the in-situ measurement of the solidsolid phase transition in shocked iron, providing the first direct experimental evidence of the high-pressure structure and details of the transition process. The overall work has laid a strong foundation for dynamic in-situ materials studies.

\section{Publications}

Hawreliak, J., et al., 2005. Shock induced (alpha)-(epsilon) phase change in iron: analysis of MD simulations and experiments. 14th American Physical Society Topical Conf. Shock Compression of Condensed Matter, Baltimore, MD, July 20-Aug. 7, 2005.

UCRL-PROC-215261.

Kalantar, D. H., et al., 2005. "Direct observation of the alpha to epsilon transition in shock compressed iron via nanosecond x-ray diffraction." Phys. Rev. Lett. 95, 075502.

UCRL-JRNL-211109.

Kalantar, D. H., et al., 2005. Direct observation of the alpha-epsilon transition in shocked single crystal iron. 14th American Physical Society Topical Conf. Shock Compression of Condensed Matter, Baltimore, MD, July 20-Aug. 7, 2005. UCRL-CONF-214972.

Kalantar, D. H., et al., 2005. In-situ diffraction measurements of lattice response due to shock loading, including direct observation of the alpha-epsillon phase transition in Fe. Hypervelocity Impact Symp., Lake Tahoe, CA, Oct. 9-13, 2005. UCRL-ABS-209092.

Wark, J. S., et al., 2005. Picosecond x-ray diffraction from laser-shocked copper and iron. 14th American Physical Society Topical Conf. Shock Compression of Condensed Matter, Baltimore, MD, July 20-Aug. 7, 2005. UCRL-CONF-215010. 


\title{
Nonequilibrium Phase Transitions
}

\author{
Andrew Ng \\ 04-ERD-108
}

\begin{abstract}
Exploring nonequilibrium phase transitions is a scientific frontier that holds promises for discovering new phases, metastable states, chemical reaction pathways, and biological functioning processes. This project will conduct the first systematic study of phase transitions in an extreme, nonequilibrium regime to examine lattice disordering and melting, quantify the role of electronic excitation on phase-transition kinetics, and develop approaches in finite-temperature condensed matter for constructing an equation of state (EOS). The project will use measurements to correlate optical and structural properties under ultrafast laser excitation to help develop density functional theory approaches, and work will be conducted in collaboration with the University of Toronto.
\end{abstract}

We expect to obtain time-correlated data on optical and structural properties by tracking solid-liquid to liquid-plasma transitions under ultrafast excitation conditions. These data will benchmark quantum simulations based on the density functional theory approach. Success of the project will lead to new understanding of the connection between electronic (optical) and atomistic (structural) behavior, opening up possibilities of manipulating phase stability and boundary while validating new developments in theory to improve predictive power. Success in this area also will help describe the convergence of condensed matter and plasma physics, a critically missing link in basic scientific understanding.

\section{Mission Relevance}

Ultrafast optical and atomistic diagnostics for nanoscale experiments, coupled with theory development using high-performance computing, will provide increased understanding of phase transitions and kinetics for EOS data development in support of the stockpile stewardship mission.

\section{FY06 Accomplishments and Results}

In FY06, we (1) made the first determination of critical lattice energy density required for solid-plasma transition under ultrafast laser excitation, and published the findings in Physical Review Letters; (2) made the first measurement of the broadband dielectric function of warm dense gold, showing the role of intra- and inter-band transitions in the intermediate phase-these results were also published in Physical Review Letters; (3) made the first benchmark of Purgatorio calculations of electron collision time, direct current conductivity, and electron density using our single-state data; and (4) completed design and construction of the ultrafast electron gun and a novel diagnostic for measuring the electron bunch length. 


\title{
Proposed Work for FY07
}

In FY07, we plan to complete three new studies: (1) ultrafast electron diffraction (UED) measurements for ultrafast laser excitation of gold at $400 \mathrm{~nm}$ to reveal temporal evolution of lattice disorder for correlation with optical properties, (2) pump-probe measurements of reflectivity and transmissivity at $800 \mathrm{~nm}$ to determine temporal evolution of electron density induced by $400-$ and $800-\mathrm{nm}$ excitation for elucidating ionization dynamics under extreme nonequilibrium conditions, and (3) UED and broadband dielectric function measurements with $800-\mathrm{nm}$ excitation for correlation with $400-\mathrm{nm}$ excitation results, which is key to understanding the effect of electronic excitation on the solid-plasma transition. These studies will be complemented by density functional theory and Purgatorio calculations.

\section{Publications}

Ao, T., et al., 2006. "Optical properties in nonequilibrium phase transitions." Phys. Rev. Lett. 96, 055001. UCRL-JRNL-204711.

Ping, Y., et al., 2006. "Broadband dielectric function of nonequilibrium warm dense gold." Phys. Rev. Lett. 96, 255003. UCRL-JRNL-221126.

\section{Kinetic Simulation of Boundary-Plasma Turbulent Transport}

Bruce I. Cohen

04-SI-003

\begin{abstract}
This project will develop a kinetic code to model tokamak plasma boundary regions, allowing first-principles predictions of the edge transport barrier, the greatest source of uncertainty in projecting the fusion power output of next-generation tokamak reactors like the International Thermonuclear Experimental Reactor (ITER). The code will include a nonlinear kinetic description of the edge plasma in realistic three-dimensional (3D) magnetic geometry. Molecular dynamics simulations will provide improved models of plasma contamination by hydrocarbons. The kinetic edge plasma code will use efficient numerical methods to solve coupled equations in a 5D phase space on massively parallel computers, and provide software structure to support continuing physics development by multi-institution partners.

This project will deliver the world's first ab initio integrated model of the boundary region of fusion plasmas, which recent work suggests may provide an accurate method of predicting and optimizing the fusion power of ITER. A full-fidelity ITER calculation will be within the capabilities of our emerging petaclass computers, such as BlueGene. A code architecture compliant with the goals of the DOE Fusion Simulation Project (FSP) will facilitate continued development of the physics by multi-institution collaborations, allowing models developed under this project to be extended into a simulation of the full tokamak as part of the FSP. The resulting kinetic edge code will be the basis for follow-on development of kinetic edge simulation capability in the FSP.
\end{abstract}




\section{Mission Relevance}

This project supports the energy security mission of Lawrence Livermore by advancing the physics required for magnetic fusion energy through the use of high-performance computing, advanced software technology, and fundamental simulation of the properties of materials. Fusion has been a core mission for the Laboratory since its founding. This project is timely because the U.S. has rejoined ITER, and a comprehensive modeling capability is viewed as necessary for its effective use.

\section{FY06 Accomplishments and Results}

In FY06, we (1) extended the 4D code to 5D by adding a toroidal coordinate and extending data structures, solvers, input/output, and visualization tools; (2) developed the 4D/5D codes to run on parallel computers; ( 3 ) began physics studies and code validation for both codes; (4) made the 5D code accessible to new users and developers; (5) continued to evaluate and improve the algorithms; and (6) undertook computations of neoclassical transport, open-field-line confinement, and self-consistent geodesic acoustic modes. Overall, we fulfilled the important project goals, and have begun follow-on work for the Edge Simulation Laboratory, which uses our TEMPEST code as a starting point.

\section{Publications}

Cohen, R. H., et al., 2006. Collisional tests and extensions of the TEMPEST continuum gyrokinetic code. 2006 Sherwood Fusion Theory Conf., Dallas, TX, Apr. 22-25, 2006. UCRL-ABS-218159.

Cohen, R. H., et al., 2006. Continuum kinetic code for edge plasmas and the ESL project. Edge Coordinating Committee Workshop, Myrtle Beach, SC, Apr. 3-8, 2006.

UCRL-ABS-220310.

Marian, J., et al., in press. "Characterization of sputtering products in tokamak plasmafacing components by molecular dynamics." J. Appl. Phys. UCRL-JRNL-330938.

Marian, J., et al., 2006. "Simulations of carbon sputtering in amorphous hydrogenated samples." Phys. Scripta T124, 65-69. UCRL-CONF-215875.

Qin, H., et al., 2006. "General gyrokinetic equations for edge plasmas." Contrib. Plasma Phys. 46, 477. UCRL-PROC-215824.

Rognlien, T. R., et al., 2006. Simulations of 4D edge transport and dynamics using the TEMPEST gyro-kinetic code. 48th Annual Mtg. Division of Plasma Physics, Philadelphia, PA, Oct. 30-Nov. 3, 2006. UCRL-ABS-223022.

Xiong, Z., et al., 2006. A finite volume approach to Fokker-Planck collision operator in the coordinates of constants-of-motion. 2006 Sherwood Fusion Theory Conf., Dallas, TX, Apr. 22-25, 2006. UCRL-ABS-218153.

Xiong, Z., et al., 2005. Continuum kinetic simulation in a toroidal annulus using TEMPEST. 47th Annual Mtg. Division of Plasma Physics, Denver, CO, Oct. 24-28, 2005.

UCRL-POST-216501. 
Xiong, Z., et al., 2006. Verification of TEMPEST with neoclassical transport theory. 48th Annual Mtg. Division of Plasma Physics, Philadelphia, PA, Oct. 30-Nov. 3, 2006. UCRL-ABS-223035.

Xu, X. Q., et al., 2006. Continuum edge gyrokinetic theory and simulations. 21st International Atomic Energy Agency Fusion Energy Conf., Chengdu, China, Oct. 16-21, 2006. UCRL-ABS-218651.

\title{
Biological Imaging with Fourth-Generation Light Sources
}

Henry N. Chapman

05-SI-003

\begin{abstract}
We propose to develop capabilities to carry out single-molecule, atomic-resolution imaging at future x-ray free-electron laser (XFEL) facilities. Our goal is to perform groundbreaking experiments at new and existing sources to test the key concepts of single-molecule XFEL imaging, including measurement of the Coulomb explosion of particles in intense, ultrashort $x$-ray beams; lensless $x$-ray imaging beyond the radiation-damage limit; and manipulation and orientation of single particles in space and time to interact with XFEL pulses. We will crosscheck experiments with substantial modeling efforts to understand the new abilities that the XFEL will bring to biological imaging. These capabilities will allow us to determine the atomic structures of any protein.

Each of our experiments, which will be a world first and a major new result in $\mathrm{x}$ ray science in every case, will (1) determine the duration and fluence of XFEL pulses required for single-molecule imaging, (2) demonstrate reconstruction methods, and (3) demonstrate ultrahigh-resolution three-dimensional (3D) imaging of container-free particles, for which new technologies in biological sample preparation will be developed. Together, these experiments will demonstrate the extraordinary science achievable with XFELs, and the impact they will have on structure determination of biological macromolecules, protein complexes, viruses, and spores.
\end{abstract}

\section{Mission Relevance}

Improved tomography algorithms will benefit stockpile stewardship. As a specific example, diffraction imaging techniques can be applied to the study of warm dense matter, a critical regime of weapons physics. Single-molecule imaging will allow the structure of virtually any macromolecule, protein, or virus to be determined, which furthers Lawrence Livermore's missions in both biodefense and bioscience to improve human health. Our research also enhances the capabilities of the Linac Coherent Light Source, a highpriority project of the DOE Office of Science, in support of the Laboratory's mission in breakthrough science and technology. 


\section{FY06 Accomplishments and Results}

In FY06, we carried out groundbreaking experiments at the Hamburg Vacuum UltravioletFree-Electron Laser (VUV-FEL), and performed the first-ever demonstration of coherent $\mathrm{x}$-ray imaging with a single 30 -fs FEL pulse, demonstrating a crucial technological milestone for single-molecule imaging. In addition, we invented and demonstrated the method of femtosecond time-delay x-ray holography, and have filed a record of invention. This allowed us to perform a direct time-resolved measurement of particle damage in an intense FEL pulse, with a resolution of $3.5 \mathrm{fs}$. We found that particles explode at a slower rate than expected from hydrodynamic models. We also developed an efficient particleinjection apparatus, and have carried out computer simulations on Thunder to determine the effect of injection on molecular structure.

\section{Proposed Work for FY07}

In FY07, we will (1) continue our program of ultrafast $x$-ray science at the VUV-FEL-our primary experiment will be the first-ever ultrafast imaging of injected particles, thereby establishing single-molecule diffraction on an experimental footing; (2) measure the dependence of particle coherent scattering on pulse fluence, to validate our hydrodynamic models; (3) continue development of time-delay holography, and demonstrate this in an off-axis geometry to holographically reconstruct the prompt and delayed images; and (4) perform imaging of biological samples beyond the radiation damage limit, and test 3D imaging of reproducible samples. Our models of damage and particle injection will be extended, and results acquired after running these simulations on supercomputers.

\section{Publications}

Bogan, M., 2006. Femtosecond pulse vacuum ultraviolet free electron laser diffraction of polystyrene nanoparticles. Particles 2006-Medical/Biochemical Diagnostic, Pharmaceutical, and Drug Delivery Applications of Particle Technology, Orlando, FL, May 13-16, 2006. UCRL-PRES-221249.

Bogan, M., 2006. Solving the enigma of gas phase biomolecular structure: single biomolecule/nanoparticle injection technology for free electron laser diffraction imaging. American Chemical Society 232nd National Mtg. and Exposition, San Francisco, CA, Sept. 10-14, 2006. UCRL-PRES-224433.

Cavalieri, A. L., et al., 2006. "Clocking femtosecond x rays." Phys. Rev. Lett. 94, 114801. UCRL-JRNL-207122.

Chalupski, J., et al., 2006. Ablation of organic molecular solids by focused soft $x$-ray freeelectron laser radiation. 10th Intl. Conf. X-Ray Lasers, Berlin, Germany, Aug. 21-25, 2006. UCRL-CONF-225006.

Chapman, H. N., 2005. Three-dimensional coherent diffraction $x$-ray imaging at $10 \mathrm{~nm}$ resolution. Advanced Light Source Users' Meeting, Berkeley, CA, Oct. 9-11, 2005. UCRL-PRES-213954. 
Chapman, H. N., 2006. Ultrafast coherent diffraction imaging with a soft $x$-ray freeelectron laser. National Synchrotron Light Source and the Center for Functional Nanomaterials Joint Users' Meeting, Upton, NY, May 15-17, 2006. UCRL-PRES-220916.

Chapman, H. N., 2006. Ultrafast coherent diffraction imaging with a soft-x-ray freeelectron laser. Gordon Research Conf. Multiphoton Processes, Tilton, NH, June 11-16, 2006. UCRL-PRES-220916.

Chapman, H. N., 2006. Ultrafast coherent diffraction imaging with $x$-ray free-electron lasers. 28th Intl. Free Electron Laser Conf., Berlin, Germany, Aug. 27-Sept. 1, 2006. UCRL-PROC-223922.

Chapman, H. N., et al., in press. "Femtosecond diffractive imaging with a soft-x-ray freeelectron laser." Nat. Phys. UCRL-JRNL-219848.

Chapman, H. N., et al., 2006. "High-resolution ab initio three-dimensional x-ray diffraction microscopy." J. Opt. Soc. Am. 23, 1179. UCRL-JRNL-214796.

Chapman, H. N., et al., 2006. Ultrafast soft $x$ ray scattering of polystyrene nanoparticles. Berkeley Nanotechnology Forum 2006, Nanotechnology: Living up to the Promise, Berkeley, CA, Apr. 15, 2006. UCRL-POST-220434.

Hau-Riege, S. P., 2006. The role of damage in diffraction imaging using free-electron lasers. 12th Intl. Workshop Radiative Properties of Hot Dense Matter, Albufeira, Portugal, Sept. 11-15, 2006. UCRL-PRES-224352.

Hau-Riege, S. P., 2006. Single-particle diffraction imaging using free-electron lasers. X-Ray Free-Electron Lasers: Challenges for Theory, Cambridge MA, June 19-21, 2006. UCRL-PRES-222449.

Lindenberg, A. M., et al., 2006. "Atomic-scale visualization of inertial dynamics." Science 308, 392. UCRL-JRNL-210233.

Marchesini, S., 2006. First results on ultrafast coherent diffraction imaging using a freeelectron laser. Advanced Photon Source 2006 Users Mtg., Argonne, IL, May 1-5, 2006. UCRL-PRES-221311.

Marchesini, S., 2006. Imaging with coherent x-rays. School of Scientific Applications of Coherent X-Ray Radiation, Hamburg, Germany, Mar. 1-2, 2006. UCRL-PRES-220693.

Marchesini, S., et al., 2006. "Phase aberrations in diffraction microscopy." IPAP Conf. Series 7, Proc. 8th Intl. Conf. X-Ray Microscopy, pp. 380-382. UCRL-PROC-215873.

Marchesini, S., et al., 2006. "Progress in three-dimensional coherent x-ray diffraction imaging." IPAP Conf. Series 7, Proc. 8th Intl. Conf. X-Ray Microscopy, pp. 353-356. UCRL-PROC-215874.

Starodub, D., et al., 2006. "Damped and thermal motion of laser-aligned hydrated macromolecule beams for diffraction." J. Chem. Phys. 123, 244304. UCRL-JRNL-215872. 


\title{
Physics from the MIPP Experiment
}

\author{
Peter D. Barnes 05-ERD-007
}

\begin{abstract}
This project will deliver the essential physics cross sections needed for two applications: (1) particle production and scattering cross sections relevant to future proton radiography facilities and (2) accurate physics models of the Neutrinos at the Main Injector (NuMI)Main Injector Neutrino Oscillation Search (MINOS) neutrino beam at Fermilab, which will enable high-accuracy neutrino measurements. The project will support analysis of the required physics data taken by the Fermilab E907 Main Injector Particle Production (MIPP) experiment, which can measure particle production and total cross sections from proton, pion, and kaon beams from 5 to $120 \mathrm{GeV} / \mathrm{c}$ momentum on nuclear targets from hydrogen to uranium, and on the Fermilab NuMI beam target for the MINOS experiment.
\end{abstract}

The project will determine the cross sections with $2 \%$ relative and $5 \%$ absolute error. The data obtained for NuMI-MINOS will allow a reduction of the neutrino spectrum systematic error (the dominant systematic error) from $10 \%$ to approximately $2 \%$. The chargedparticle cross sections obtained for radiography will allow high-accuracy modeling and analysis of past experiments at Brookhaven National Laboratory and future experiments at Advanced Hydro Facility-class facilities. The project will result in publication of the cross sections, which will be entered into Lawrence Livermore databases and made available for simulation codes, and will enhance strong collaborations with DOE Office of Sciencesupported national laboratory and university groups.

\section{Mission Relevance}

The project will deliver total cross sections, particle-production cross sections, and scattering distributions from proton, pion, and kaon interactions in the momentum ranges and with targets relevant to the Laboratory's stockpile stewardship mission. Simultaneously, these precision measurements will also enhance the physics yield from a DOE Office of Science priority, the MINOS experiment.

\section{FY06 Accomplishments and Results}

In FY06, we conducted experiments that acquired 31 million physics events, including (1) 8.2 million events with solid nuclear targets from beryllium to uranium, with pion, kaon, and proton beams from 5 to $85 \mathrm{GeV}$ and a $120-\mathrm{GeV}$ proton beam; (2) 7.1 million events with liquid hydrogen; (3) 1.8 million events with the NuMI target; and (4) 14 million events for a high-precision kaon mass measurement. We also began developing our data-reconstruction software, which included first-pass reconstruction for the time-projection chamber and hit-finding, and track-matching for the entire experiment. We implemented an improved time-projection chamber cluster fitter, which will enable proper calibrations and significantly improved particle identification, and began calibration of the Cerenkov detector. 


\title{
Proposed Work for FY07
}

In FY07, we will (1) complete calibration and particle identification analysis for the detectors, (2) reconstruct the entire data set, (3) perform acceptance and efficiency studies, and (4) generate the summary data set of produced particle four-vectors. In addition, we will perform the final cross-section analysis, insert the data into the Livermore data tables so they are accessible for Livermore simulation codes, and publish papers on our results.

\section{Neutron Capture Cross-Section Measurements at DANCE}

Winifred E. Parker 05-ERD-011

\begin{abstract}
Important nuclear cross sections ( $n$,gamma) are not well known and are very difficult to model. Certain isotopes important to the astrophysical s-process have no experimental measurements at all. Some isotopes of interest to stockpile stewardship have measurements that disagree with each other. We propose to perform neutron capture cross-section measurements using radioactive targets, which is a novel and high-risk technique, as well as using the Detector for Advanced Neutron Capture Experiments (DANCE) detector array and the white neutron source at Los Alamos National Laboratory. Each measurement will be performed in two stages: target preparation and cross-section measurement. In addition to making stable targets, we will develop the ability to make radioactive targets.
\end{abstract}

We expect to increase knowledge of the properties of unstable nuclei, including leveldensity information and statistical theory of gamma-ray decay. In astrophysics, the impact of this work will be significant because current models give results that differ from global isotopic abundances by an order of magnitude. No measurements have been performed on radionuclides that we propose to study. Furthermore, modern approaches involving quantification of margins and uncertainties require improved physics input. The largest piece of missing cross-section information is the capture-gamma cross sections. If the technique involving unstable nuclei as targets is successful, the problems of europium (Eu) and gadolinium (Gd) cross sections for stockpile stewardship calculations may be solved.

\section{Mission Relevance}

This project supports the national security mission. If successful, the unstable target approach will directly affect stockpile science as well as nuclear and astrophysical science. It will open the window to experimental data that apply directly to stockpile certification. Many detectors were added to nuclear devices to determine details of the nuclear detonation and, for many of the production radionuclides, cross sections for their destruction are unknown. 


\title{
FY06 Accomplishments and Results
}

In FY06, we (1) completed the ${ }^{151,153} \mathrm{Eu}$ data analysis and are finalizing the cross-section results; (2) described the statistical theory of gamma-ray decay of an excited nucleus; (3) performed ( $n$,gamma) measurements on three Gd targets at DANCE and began data analysis; (4) created targets by electroplating material onto beryllium foils for ${ }^{157,160} \mathrm{Gd}$ targets as well as a radioactive americium (Am) target, which was our first radioactive target; (5) performed capture cross-section measurements at DANCE on ${ }^{157} \mathrm{Gd}$ and ${ }^{242 \mathrm{~m} A m \text {; }}$ and (5) began data analysis on ${ }^{242 \mathrm{~m} A m}$ and ${ }^{157} \mathrm{Gd}$, performing the first ever ( $\mathrm{n}$,gamma) measurement on ${ }^{242 \mathrm{~m}} \mathrm{Am}$.

\section{Proposed Work for FY07}

In FY07, we will (1) collect ( $n$,gamma) data for ${ }^{160} \mathrm{Gd}$ and nat $\mathrm{Gd}$; (2) complete analysis of the Gd cross-section data; (3) look for evidence in the Gd data for the scissors resonance found in the Eu data (this is a low-energy resonance in the radiative strength function); (4) develop an improved ${ }^{242 \mathrm{~m} A m}$ target; (5) make a second radioactive target, using possibly ${ }^{153} \mathrm{Gd}$ or ${ }^{243} \mathrm{Am}$; (6) investigate making ${ }^{151} \mathrm{Gd},{ }^{154,155} \mathrm{Eu}$, samarium-151, or uranium

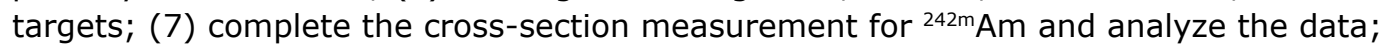
and (8) finalize our results on the Eu, Gd, and Am data for publication in peer-reviewed journals. By the end of FY07, we will have accurate ( $n$,gamma) cross sections for use in stockpile stewardship and astrophysics.

\section{Publications}

Agvaanluvsan, U., et al., 2006. Progress on the europium neutron-capture cross section study. 19th Intl. Conf. Application of Accelerators in Research and Industry, Fort Worth, TX, Aug. 20-25, 2006 UCRL-PROC-225343.

\section{Hysteresis and Kinetic Effects during Liquid-Solid Transitions}

\author{
Frederick H. Streitz 05-ERD-014
}

\begin{abstract}
Our goals for this project are threefold: (1) gain insight into the kinetics of solid-solid and liquid-solid transitions, (2) determine the shape of the high-pressure and high-temperature melt line for metals in general, and (3) understand how hysteresis during multiple crossings of the melt line affect the liquid-solid transition. Acquiring this information is the first step towards developing a dynamic equation-of-state (EOS) model. These goals will be accomplished by investigating the high-pressure solidification of prototypical materials, such as iron, bismuth, tin, and water, through combinations of shock and isentropic compression in gas guns using graded-density impactors. In addition to these experiments, companion calculations will be performed at the hydrodynamic and atomic scales.
\end{abstract}


The success of this project would represent the first experimental measurement of the time scale for phase transitions under high pressure and temperature. Specifically, it will produce the first-ever look at the kinetics of pressure-induced transitions in metals. The insights gained into the kinetics of solid-solid transitions and solidification represent vital input for the validation of several simulation codes for stockpile stewardship, including the incorporation of a next-generation multiphase, multitable EOS in hydrodynamic codes. In addition, we will utilize for the first time simultaneous surface-sensitive and volume-sensitive measurements to probe the dynamics of the transition.

\section{Mission Relevance}

This project supports the national security mission through increased understanding of the dynamics of materials under extreme conditions, particularly rapid resolidification in metals under conditions of high pressure and temperature, which is specifically applicable to stockpile science. This project also contributes to the Laboratory's mission in basic science.

\section{FY06 Accomplishments and Results}

In studying solid-solid transitions in bismuth, we used conductivity and photonic Doppler velocimetry (PDV) as diagnostics and, unexpectedly, found evidence of velocity fluctuations at the bismuth-sapphire interface as the shock traverses the sample. Although this new direction prevented us from exploring solidification and hysteresis effects, the resulting data-the first simultaneous measurement of a volume and surface response in a material undergoing a phase change-may be the first look into the dynamics of a mixed phase. Overall, this project succeeded in proving that conductivity is a viable measurement with which to investigate phase transitions in some metals, especially when coupled with PDV.

\section{Publications}

Chau, R., and F. H. Streitz, 2006. Kinetics of solid-to-solid phase transitions in bismuth. American Physical Society March Mtg. 2006, Baltimore, MD, Mar. 13-17, 2006. UCRL-ABS-217485.

\section{Optical Properties as a Real-Time in situ Materials Diagnostic at Extreme Conditions}

Jeffrey $H$. Nguyen 05-ERD-030

\section{Abstract}

Although characterizing the state of materials at subnanosecond time resolution is critical in experiments studying time evolution of phase transitions, realistic diagnostics are scarce. This project will develop the use of optical properties as a diagnostic tool to obtain 
materials information, including inferred crystal structure, in real time from experiments at LLNL's gas gun. We propose to measure accurate optical properties and to leverage the Laboratory's Advanced Simulations and Computing (ASC) calculations of these same quantities. Direct comparison between theory and experiment will enable association of the observed optical properties with other materials properties by establishing spectroscopic fingerprints for each crystalline phase as well as the liquid.

We expect to develop a diagnostic capable of measuring in situ the structure of a material in real time as it is subjected to extreme temperatures and pressures in dynamic experiments at gas guns (at LLNL's Joint Actinide Shock Physics Experimental Facility and at Los Alamos National Laboratory), high-power lasers, and Sandia's Z Machine. Moreover, the real-time nature of the diagnostic will enable us to infer structural quantities while they are changing. This information is not only essential for understanding experiments conducted on very disparate time scales, from subnanosecond laser shocks to minutes or hours in a diamond anvil cell, but is crucial to developing a theory of dynamic phase transitions for incorporation into existing hydrodynamic simulation codes.

\section{Mission Relevance}

This project will develop a real-time, in situ diagnostic to characterize crystal structures with subnanosecond resolution for actinide research, in support of the stockpile stewardship mission. This diagnostic will be portable to experiments at other gas guns, high-power lasers, and Sandia's Z Machine to support basic science research. The project also leverages ASC theoretical effort on optical properties for stockpile stewardship.

\section{FY06 Accomplishments and Results}

In FY06, we measured the complex optical properties of iron, bismuth, and tin as they undergo phase transitions during shock loading. We also applied the same diagnostic to measure the time scale of the phase transition as water solidifies under quasi-isentropic compression. These results were obtained at a wavelength of $532 \mathrm{~nm}$, which was sufficient to detect phase transitions. However, to identify in situ crystal structures, we proceeded to measure optical properties of gold and aluminum with white light at ambient conditions. Preparations were made for experiments to measure broad-band optical properties at extreme conditions. On the theoretical side, we extended our earlier work on aluminum to higher pressures and temperatures to correlate with planned shock-melting experiments.

\section{Proposed Work for FY07}

In FY07, we propose to make broadband measurements of the optical properties of crystals to identify their structures at extreme temperature-pressure conditions. Initial experiments will include measurements of broadband, complex optical properties of aluminum and iron (and possibly bismuth and tin) under shock-loading conditions. If successful, we plan to carry out similar measurements under tailored dynamic compression. These experiments may enable examination of the time scale of phase transitions. In addition, we propose to calculate the corresponding optical properties as a function of temperature and pressure. We will then be able to identify crystal structures at extreme pressure-temperature conditions in real time during gas-gun experiments. 


\section{Publications}

Nguyen, J. H., 2006. Dynamic solidification of water. Gordon Research Conf., Research at High Pressure, Biddeford, ME, June 25-30, 2006. UCRL-ABS-222369.

Nguyen, J. H., et al., 2006. Crystal structure characterization using optical properties. AIRAPT Intl. Conf. High Pressure Science and Technology, Karlsruhe, Germany, June 2006. UCRL-ABS-208816.

Nguyen, J. H., et al., 2006. Dynamic solidification of water. Gordon Research Conf., Research at High Pressure, Biddeford, ME, June 25-30, 2006. UCRL-POST-222394.

Nguyen, J. H., et al., 2006. High temperature high pressure emissivities. 14th APS Topical Conf. Shock Compression of Condensed Matter, Baltimore, MD, Aug. 5, 2005. UCRL-PRES-214206.

Nguyen, J. H., et al., 2005. Crystal structure characterization using high pressure-temperature optical properties. 2005 APS March Mtg., Los Angeles, CA, Mar. 21-25, 2005. UCRL-ABS-208363.

Nguyen, J. H., et al., 2005. Crystal structure characterization using high pressure-temperature optical properties. 2005 APS March Mtg., Los Angeles, CA, Mar. 21-25, 2005. UCRL-ABS-208363.

Nguyen, J. H., J. R. Patterson, and N. C. Holmes, 2006. Dynamic ellipsometry under extreme conditions. 2006 APS March Mtg., Baltimore, MD, Mar. 13-17, 2006. UCRL-ABS-217433.

Nguyen, J. H., J. R. Patterson, and N. C. Holmes, 2006. High-temperature, high-pressure emissivities. 14th APS Topical Conf. Shock Compression of Condensed Matter, Baltimore, MD, Aug. 5, 2005. UCRL-ABS-214205.

Nguyen, J. H., et al., 2006. Crystal structure characterization using high pressure-temperature optical properties. 2005 APS March Mtg., Los Angeles, CA, Mar. 21-25, 2005. UCRL-ABS-208363.

Patterson, R., et al., 2005. Optical properties of shock-compressed materials. 14th APS Topical Conf. Shock Compression of Condensed Matter, Baltimore, MD, Aug. 5, 2005. UCRL-PRES-214217.

Patterson, R., J. Nguyen, and N. Holmes, 2006. Application of ellipsometry to shockcompressed materials. Gordon Research Conf., Research at High Pressure, Biddeford, ME, June 25-30, 2006. UCRL-POST-222370.

Patterson, R., J. Nguyen, and N. Holmes, 2006. Application of ellipsometry to shockcompressed materials. 2006 Gordon Research Conf., Research At High Pressure, Biddeford, ME, June 25-30, 2006. UCRL-ABS-222373.

Patterson, R., J. Nguyen, and N. Holmes, 2006. Application of ellipsometry to shockcompressed materials. 2006 APS March Mtg., Baltimore, MD, Mar. 13-17, 2006. UCRL-ABS-217434. 


\title{
Advanced Studies of Hydrogen at High Pressures and Temperatures
}

\author{
William J. Evans 05-ERD-036
}

\begin{abstract}
The goal of this project is to study hydrogen at high pressures (megabars) and temperatures (thousands of kelvin). Properties of high-pressure hydrogen in this regime are important to a range of basic and applied sciences, including condensed-matter theory, modeling efforts, planetary science, and hydrogen energy storage. High-pressure hydrogen is the subject of intensive theoretical and experimental studies, both static and dynamic. The proposed high-pressure and high-temperature experiments will bridge the gap between static and dynamic experiments. We propose to apply our recent advances in state-of-the-art high-pressure, $\mathrm{x}$-ray, laser, and spectroscopic capabilities. These studies will address important issues such as the equation of state at high pressures and temperatures, metallization, phase lines, and novel phase transitions.
\end{abstract}

The rich physics in the regime between dynamic and static studies are targeted by this proposal. Shock wave studies $(150 \mathrm{GPa}, 3000 \mathrm{~K})$ find a liquid metallic phase, while static work $(0-300 \mathrm{GPa}, 77 \mathrm{~K})$ has identified only solid insulating states. The goal is to find the pressure and temperature states bridging these regimes that contain transitions in properties to reconcile these disparate results-that is, the phase lines for melting, metallization, and dissociation. Further, we seek to measure the melt line, liquid-liquid (molecular-nonmolecular) transition, and the metallic fluid state predicted by theory. Such discoveries in hydrogen would impact our understanding for defense applications, Jovian planets, and hydrogen energy storage.

\section{Mission Relevance}

The deliverables of equation of state and phase transitions of high-pressure and hightemperature hydrogen directly address needs in stockpile stewardship (specifically, the extreme dynamics of materials) and hydrogen energy storage, in support of LLNL's national and energy security missions.

\section{FY06 Accomplishments and Results}

In FY06, using a nonlinear optical technique (coherent anti-Stokes Raman spectroscopy), we measured the band gap of deuterium to be $4.7 \mathrm{eV}$ at $165 \mathrm{GPa}$-the first time this measurement has ever been performed at such high pressure. These results benchmark theoretical work and can be used to predict the metallization pressure of hydrogen. We also developed a high-pressure gas-loading system that enables us to make measurements of the hydrogen melt curve. We began experiments to measure the melt curve at high pressures. Our work on the band gap was presented as an invited poster at a high-visibility computations conference. 


\title{
Proposed Work for FY07
}

In FY07, we will study the structure and phase transitions in the liquid phase of hydrogen. For these measurements, we will use heating techniques (laser and resistive), optical spectroscopies, and x-ray scattering. Building on our work on the solid phase and its melt, we will develop further understanding of liquid phase properties. In addition to basic scientific parameters of the liquid (density and pair distribution function), we also expect to see molecular-atomic transitions at sufficiently high temperature.

\section{Publications}

Evans, W. J., et al., 2006. Fundamental properties of hydrogen at high pressures: bandgap and melt curve. Gordon Research Conf., Research at High Pressure, Biddeford, ME, June 2006. UCRL-POST-222340.

Evans, W. J., et al., 2006. Fundamental properties of hydrogen at high pressures: bandgap and melt curve. Gordon Research Conf., Research at High Pressure, Biddeford, ME, June 2006. UCRL-ABS-221022.

\section{Hydrodynamic, Atomic Kinetic, and Monte Carlo Radiation Transfer Models of the X-Ray Spectra of Compact Binaries}

\author{
Christopher W. Mauche 05-ERD-044
}

\begin{abstract}
This project will model the $\mathrm{x}$-ray spectra of compact binaries (white dwarf, neutron star, and black hole) by constructing highly detailed, 3-dimensional (3D) hydrodynamic models of the plasma flow in these binaries, calculating the x-ray spectra of every point in the flow, and transporting this radiation through the flow to the observer. These activities will be accomplished with the FLASH hydrodynamic package, LLNL atomic models, and our Monte Carlo radiation transfer code, using the massively parallel computing resources of LLNL. These models will be used to interpret the existing Chandra and XMM-Newton and future Astro-E2, Constellation-X, and XEUS high-resolution $\mathrm{X}$-ray spectra of compact binaries.

If successful, the project will produce highly detailed, 3D, time-dependent hydrodynamic models of the flow of plasma in compact binaries. These models will be used to produce the first realistic $x$-ray spectral models of compact binaries with sufficient detail to predict relative and absolute line strengths and line shapes as a function of binary phase. Although focused initially on high-mass $x$-ray binaries, the capabilities we will develop will be applicable to all types of $x$-ray sources dominated by photoionized plasmas. Therefore, the results will have far-reaching and long-term importance in $\mathrm{x}$-ray astrophysics.
\end{abstract}




\title{
Mission Relevance
}

By providing hydrodynamic, atomic kinetic, and Monte Carlo radiation transfer models of the $x$-ray spectra of compact binaries, this project contributes to radiation-hydrodynamic codes that simulate nuclear explosives for the stockpile stewardship mission.

\section{FY06 Accomplishments and Results}

During FY06, significant progress was made toward the goal of performing realistic computer models of the radiation-hydrodynamic flows in high-mass $x$-ray binaries, the resulting $x$-ray spectra, and comparison to data. Specifically, we (1) added non-inertial forces to FLASH; (2) performed 2D hydrodynamic simulations, calculating local x-ray emissivities and global spectra; (3) developed and began implementing a more detailed treatment of radiatively driven photoionized winds; (4) began an analysis of the Chandra x-ray spectra of Vela X-1; (5) extended our calculations to 3D; 6 ) began modifying our code to perform Monte Carlo calculations of x-ray radiation transfer; (7) hired a postdoc; and (8) began to investigate ways to adapt our code and what we have learned about radiation hydrodynamics to national-security-relevant activities.

\section{Proposed Work for FY07}

During FY07, we plan to (1) complete and apply the more detailed treatment of radiatively driven photoionized winds; (2) conduct long, high-spatial-resolution 3D FLASH simulations of Vela X-1; (3) complete and apply the Monte Carlo radiation transfer calculations to the resulting 3D structures; (4) complete the analysis of the Chandra x-ray spectra of Vela $\mathrm{X}-1 ;(5)$ make detailed comparisons between the model and observed $\mathrm{x}$-ray spectra of Vela X-1; $(6)$ present results at astrophysics conferences and submit papers to major astrophysics journals; (7) begin work on other high-mass $x$-ray binaries and on other related cosmic $x$-ray sources.

\section{Publications}

Mauche, C. W., D. A. Liedahl, and T. Plewa, 2006. "Hydrodynamical and spectral simulations of HMXB winds." Bull. Am. Astron. Soc., 38(3), 333. UCRL-ABS-223074.

\section{The Opacity of the Solar Interior}

\author{
Pravesh K. Patel 05-ERD-045
}

\begin{abstract}
For this project, we will make experimental measurements of the opacity of materials in the high-density, high-temperature regime of stellar physics. Opacity, which governs the transport of radiation through a material, is of fundamental importance in plasmas at very high energy densities and radiation-dominant regimes. However, opacity calculations are extremely complex, and little or no data exist for benchmarking models at high energy
\end{abstract}


densities. This project will obtain such data for the first time by utilizing a new set of ultrashort-pulse heating and $\mathrm{x}$-ray backlighting techniques on next-generation petawattclass laser facilities. Measurements will be made at solid densities of 2.3 to $8.9 \mathrm{~g} / \mathrm{cm}^{3}$ and at temperatures ranging from 20 to $100 \mathrm{eV}$.

At the conclusion of this project we expect to have obtained frequency-resolved opacity data for a set of heavy elements (carbon, nickel, and iron) abundant in the solar interior. For accurate comparison with modeling, we will also independently characterize the density, temperature, and uniformity of the heated samples. The results will access plasma densities at least two orders of magnitude higher than any previous measurements and will provide the first experimental data able to validate Lawrence Livermore opacity codes in this high-temperature, high-density regime.

\section{Mission Relevance}

This project will greatly enhance our understanding of the physics of high-energy-density plasmas. Improvements to opacity codes in this radiation-dominant regime are of central importance to LLNL's mission in stockpile stewardship.

\section{FY06 Accomplishments and Results}

In FY06, we (1) transitioned the experimental campaign to the Laboratory's new petawattclass Titan laser, performing the commissioning and first-light experiment; (2) successfully commissioned a number of new diagnostics including two-color extreme-ultraviolet imagers to measure isochoric electron and proton heating, as well as proton spectrometers and an x-ray spectrometer; and (3) obtained an excellent initial set of data demonstrating the ability to generate intense, high-quality proton beams, as well as perform proton heat tests on gold-grid targets. This required that we consistently focus the laser and heat sub-150- $\mu \mathrm{m}$ targets to be used as the thermal x-ray backlighter for the absorption measurements.

\section{Proposed Work for FY07}

In FY07, we will perform the first integrated experiments bringing together all of the techniques developed and perfected during the first two years of this project. These will be frequency-resolved opacity measurements, initially for low-atomic-number materials (aluminum) at approximately $20 \mathrm{eV}$, and later extended to mid-atomic-number astrophysically relevant elements (nickel and iron) at temperatures of $100 \mathrm{eV}$ and at solid density.

\section{Publications}

Patel, P. K., et al., 2006. "Integrated laser-target interaction experiments on the RAL petawatt laser." Plasma Phys. Contr. Fusion 47, B833. UCRL-JRNL-225356. 


\title{
Split-Beam, Short-Pulse Final Optics and Characterization for High-Energy Short Pulses
}

Igor Jovanovic 05-ERD-060

\begin{abstract}
This project will help to develop critical technology for enabling high-energy, short-pulse laser systems on large-scale neodymium-glass lasers in a chirped-pulse amplification scheme with a compact, folded compressor in a split-beam geometry. Such a system could significantly enhance current and planned high-energy-density (HED) science and inertial fusion facilities by enabling the capability to generate high energy $x$-ray radiographs of laser driven experiments. The goals of this project are (1) evaluation of final focus and alignment of split-beam short pulses and (2) development of precision metrology for determining the alignment, in both space and time, of a focused split-beam pulse that can be implemented on high-energy-density and inertial-fusion-class laser systems.

We will develop several key technology components for the back end of a high-energy, short-pulse, split-beam laser system and also perform prototype testing of those components. This project should solve most the outstanding technology challenges associated with focusing and characterizing such laser systems. The split-beam concept represents a new paradigm for achieving very-high-power, short-pulse laser operation that will enable the deployment of both a multiple-time-frame and a multiple-view, highenergy, $\mathrm{K}$-alpha radiographic diagnostic capability. This work is also expected to result in multiple publications and new intellectual property.
\end{abstract}

\section{Mission Relevance}

New back-end components and advanced metrological methods support LLNL's national and energy security missions by furthering many areas of HED and inertial confinement fusion. These include compression of multikilojoule pulses in chirped-pulse amplification systems for use in x-ray radiography, scaling of high-energy, short-pulse systems to $\sim 100 \mathrm{~kJ}$ using split-beam configurations with application to fast ignition, and development of a novel pulse metrology capability for high-energy split-beam short-pulse (HESBSP) technology with applications to future fusion-class lasers.

\section{FY06 Accomplishments and Results}

In FY06 we (1) completed the conceptual design and constructed a large section of the table; (2) commissioned the diagnostics test laser and pulse-transport design with 3.3-J energy; (3) commenced with tests of novel, sensitive HESBSP metrology, including the demonstration of a 175-ps contrast measurement window, pulse suppression by a factor of 100 using a water cell optical limiter, and interferometric accuracy for inter-split-beam timing measurement using spectrally dispersed two-beam interferometery; (4) designed a new device-spectral phase interferometry for direct electric-field reconstruction (SPIDER)-that can measure near-transform-limited HESBSPs; and (5) scaled the compressor-alignment techniques to a full-size compressor. 


\title{
Proposed Work for FY07
}

In FY07 we will (1) complete construction of the vacuum compressor and use it to compress pulses, generate a split beam, and transport it to the diagnostics; (2) complete the temporal characterization system by constructing and testing a diagnostics pulse tweaker, the SPIDER device tailored for HESBSPs, and a third-order cross-correlator with high dynamic range, and by improving pulse suppression for photodiode pre-pulse measurement; (3) integrate our diagnostics into the HESBSP system and conduct a complete single-shot test; and (4) create a complete, validated scheme for compact, folded compressor alignment.

\section{Publications}

Crane, J. K., et al., 2006. Status of the arc-a quad of high-intensity beam lines at the National Ignition Facility. Intl. Conf. Ultrahigh Intensity Lasers, Cassis, France, Sept. 25-29, 2006. UCRL-ABS-221733.

Jovanovic I., et al., 2006. Development of diagnostics for high-energy petawatt pulses. Intl. Conf. Ultrahigh Intensity Lasers, Cassis, France, Sept. 25-29, 2006.

UCRL-ABS-222193.

Lucianetti, A., et al., 2006. Spectrally dispersed two-beam interferometer for the coherent addition of pulse energy. Conference on Lasers and Electro-Optics, Long Beach, CA, May 21-26, 2006. UCRL-PRES-221593.

Siders, C. W., et al., 2006. Scaling to ultrahigh intensities by high-energy petawatt beam combining. Intl. Conf. Ultrahigh Intensity Lasers, Cassis, France, Sept. 25-29, 2006. UCRL-ABS-222194.

\section{Precision Split-Beam, Short-Pulse, Seed Laser Technology}

Jay W. Dawson

\author{
05-ERD-061
}

\section{Abstract}

High-energy-density (HED) science and inertial fusion are of strategic importance to national security. A significant enhancement of national HED capability would result by adding one or more high-power, short-pulse laser beams to existing and planned HED facilities. This proposal will provide critical technology that would enable such additions. Existing low-energy, seed laser technologies for high-energy, short-pulse lasers are unreliable and ill-suited for use with large-scale (e.g., $10 \mathrm{~kJ}$ per beam line) laser systems. This project will study, by constructing integrated subsystems, all issues required for the production of multiple-aperture, separately timed and dispersed seed pulses suitable for amplification in large-scale, neodymium-glass laser systems.

This project will develop seed laser technology for scaling high-energy, short-pulse laser beams on $40-\mathrm{cm}$-aperture, neodymium-glass laser systems across the DOE complex. 
This technology will enable the spatial multiplexing of amplified short pulses in the same beam line, a new paradigm for achieving very high power in short-pulse lasers. In addition to increasing the overall short-pulse energy extractable from a single beam line, this technology may significantly increase the utility of a short-pulse system by allowing multiple-time-frame $x$-ray imaging and other applications. This research is likely to result in numerous patents and publications.

\section{Mission Relevance}

The DOE complex has a large, nationwide investment in high-energy lasers, particularly high-energy, short-pulse lasers for research in high-field, laser-matter interactions. This project, which to our knowledge is the only one in the DOE complex pursuing critical technology on the use of fiber lasers for short-pulse front ends, supports Lawrence Livermore's national security mission, specifically stockpile stewardship.

\section{FY06 Accomplishments and Results}

In FY06, we (1) completed design of the critical components of the front-end prototype; (2) assembled the mode-locked, fiber-laser master oscillator and demonstrated that it can meet all of the target specifications, including less than 12-ps root-mean-square timing; (3) completed assembly of the final split-beam fiber amplifier components, and achieved encouraging results in preliminary tests; (4) completed construction of the remaining components; (5) constructed a robust 25-nJ, mode-locked fiber oscillator, which should be scalable to higher pulse energies and will greatly simplify future front-end systems;

(6) demonstrated the production of greater than 1-mJ pulses with good spectral features in our linear frequency-modulation fiber amplifier; and (7) began integrated systems testing.

\section{Proposed Work for FY07}

We plan to (1) complete integrated systems testing, (2) conduct computer-aided process engineering experiments, (3) develop and implement diagnostics within the system, and (4) conduct a field trial of the system at the National Ignition Facility.

\section{Publications}

Dawson, J. W., et al., 2006. Fiber laser front end for high energy petawatt laser systems, Intl. Conf. Ultrahigh Intensity Lasers, Cassis, France, Sept. 25-29, 2006.

UCRL-PROC-222261.

Dawson, J. W., et al., 2006. High energy mode locked fiber oscillators for high contrast, high energy petawatt laser seed sources. Intl. Conf. Ultrahigh Intensity Lasers, Cassis, France, Sept. 25-29, 2006. URCL-PROC-222263.

Dawson, J. W., et al., 2006. "High energy short pulse fiber laser front end for kilo-joule class CPA systems." Proc. SPIE 6102, 610214. UCRL-ABS-218610. 


\title{
Dark Matter and Dark Energy Science
}

\author{
Leslie Rosenberg 05-ERD-063
}

\begin{abstract}
Three decadal surveys have shown the importance of large-aperture synoptic survey telescopes for time-domain and cosmological studies of distant objects. We propose to develop the basic tools and techniques for investigating "dark sector" cosmological science with the next-generation of large-aperture, real-time telescopes. The critical research involves determining if extremely small distortions (shears) in images of faint background galaxies can be measured to reveal the distribution of dark matter and dark energy in the universe. If successful, this project would significantly enhance the prospects for building next-generation synoptic telescopes and greatly enhance their scientific potential.

The limiting systematic uncertainties in cosmological observations are from uncorrectable effects in the telescope point spread function (PSF) caused by the atmosphere. One anticipated result of this project will be the predicted star-corrected PSF components expected at a representative site, given the expected distribution of the atmospheric parameter. The second result will be a program to determine if atmospheric models can be extrapolated to the huge aperture and long exposure time the novel telescope would offer. This research will yield scientific publications and play a key role in determining the feasibility of building these synoptic telescopes (such as the proposed Large-Aperture Synoptic Survey Telescope and Panoramic Survey Telescope and Rapid Response System) for weak-lensing science.
\end{abstract}

\section{Mission Relevance}

The technologies required for a weak-lensing program on the scale of the next-generation instruments are directly relevant to the Lawrence Livermore's national security mission. In particular, large telescopes and gigapixel focal planes are required for future surveillance measurements. The algorithms and techniques used for weak-lensing analyses would also enhance capabilities that support nonproliferation efforts.

\section{FY06 Accomplishments and Results}

The main activity for FY06 was to analyze data taken at a large-aperture synoptic survey telescope and determine if the uncorrectable residuals from atmospheric turbulence dominate expected statistical and systematic errors. Image data taken at the 8.4-m Gemini South telescope in conjunction with simultaneous atmospheric data were analyzed. We demonstrated that even under the worst conditions encountered during the four-day campaign, residuals were comparable to shot noise from present weak lensing surveys. We further found a correlation between the ellipticity residuals and the wind direction. In addition, we compared fast Fourier versus ray-tracing methods in weak lensing analyses, and have submitted the results for publication. 


\title{
Publications
}

Asztalos, S., et al., in press. "Properties of ellipticity correlation with atmospheric structure from Gemini South." Astrophys. J. UCRL-JRNL-226988.

\section{Development of Hot, LTE-Tunable Radiation Sources for Material Science Studies and Simulating Radiation Transport in Dense Astrophysical Plasmas}

\author{
Marilyn B. Schneider 05-ERD-068
}

\begin{abstract}
A high-temperature radiation source would enable studies of materials under extreme conditions to be performed at Omega and other large-scale laser facilities. An appropriate radiation source produces hot radiation, maintains a heated material sample in local thermodynamic equilibrium (LTE), and allows good (optically thin) experimental access to the heated sample. This project is developing such a hot $\left(T_{\text {rad }}>150 \mathrm{eV}\right)$ radiation sourcethe thin back wall of a "hot" hohlraum-and the spectroscopic techniques to characterize it. This project leverages previous efforts that developed radiation sources capable of heating targets to $\sim 50 \mathrm{eV}$.

This project is producing a well-characterized hot radiation platform for studies of materials under extreme conditions. The platform enables the researchers to put a physics package in the location of the witness plate and, with the appropriate spectrometers and backlighters, to perform opacity and atomic physics studies.
\end{abstract}

\section{Mission Relevance}

This basic science research in high-energy-density physics will find applications in $\mathrm{x}$-ray spectroscopy, astrophysics, opacity, and experiments with high-power lasers that support the stockpile stewardship mission. In addition, the project contributes to the Laboratory's mission in basic science.

\section{FY06 Accomplishments and Results}

The radiation drive from the thin back wall was measured for target geometries of varying diameters and aspect ratios and for varying back wall material and thicknesses. Two shot series were conducted. In the first, radiation temperatures of 190 to $230 \mathrm{eV}$ were achieved, and an x-ray imager verified that the gold witness plate was radiatively heated. In the second shot series, a chromium witness plate was radiatively expanded to $60 \mu \mathrm{m}$ within $400 \mathrm{ps}$ and was not hit by the thin back wall. We also designed a soft x-ray spectrometer with a spectral range in the thermal region of the source $(500-1500 \mathrm{eV})$, tested it with Laboratory $\mathrm{x}$-ray sources, and calibrated it at the Electron Beam Ion Trap. This spectrometer was then put into experimental use at Omega. 


\title{
Proposed Work for FY07
}

In FY07, our spectroscopy technique will be fine-tuned to measure the time-resolved spectra and expansion (for material density) of the chromium witness plate. The experiments will be designed to mitigate the contribution to the heating of the witness plate from hot electrons, harder x-rays, and other non-LTE energy sources. This will be accomplished with a second soft $x$-ray spectrometer with higher magnification and additional available spectrometers.

\section{Publications}

Constantin, C. G., et al., 2006. "Laser-plasma interactions in high-energy density plasmas." J. de Physique IV 133, 243. UCRL-CONF-214910.

Hinkel, D. E., 2006. "Laser coupling to reduced-scale targets at NIF early light." J. de Physique IV 133, 237. UCRL-CONF-215092.

Schneider, M. B., et al., 2006. "X-ray flux and x-ray burnthrough experiments on reducedscale targets at the NIF and Omega lasers." J. de Physique IV 133, 1205. UCRL-CONF-214849.

Schneider, M. B., et al., 2006. Development of a thermal $x$-radiation source using hot hohlraums. 12th Intl. Workshop Radiative Properties of Hot Dense Matter, Albufeira, Portugal, Sept. 11-15, 2006. UCRL-ABS-222976.

Schneider, M. B., et al., in press. "Plasma filling in reduced-scale hohlraums irradiated with multiple beam cones." Phys. Plasma. UCRL-JRNL-223616.

\section{Developing the Physics Basis of Fast-Ignition Experiments at Future Large Fusion-Class Lasers}

\author{
Andrew J. Mackinnon 05-ERI-001
}

\section{Abstract}

This project will establish the physics basis, measurement techniques, and numerical designs for future integrated fast-ignition research, which is an innovative approach to achieving fusion. This research will be conducted at U.S. and foreign laser facilities, in collaboration with U.S. universities, with a team that comprises experts in fast ignition and numerical modeling. Advanced modeling of the short-pulse ignition process with particlein-code and hybrid particle-in-code will be benchmarked against experiments and used to assess short-pulse heating in the design of experiments at future, fusion-class lasers. Experiments will develop diagnostics of electron isochoric heating based on extreme ultraviolet (XUV) emission and zirconium K-alpha fluorescence, and will study heating by focused proton beams.

The deliverables include optimized hydrodynamic designs for fast-ignition targets specific to large, fusion-class lasers, with near-term scaled designs based on both electron- 
and proton-ignition tested experimentally at the Omega laser. This near-term work will provide a significant contribution to the forefront of fast-ignition research and experiments worldwide.

\section{Mission Relevance}

The work directly supports the national and energy security mission areas of stockpile stewardship and fusion energy by advancing work on short-pulse and high-energy petawatt lasers.

\section{FY06 Accomplishments and Results}

In FY06, we (1) tested a zirconium Ka fluorescence diagnostic on the Titan laser, (2) developed a hydrodynamic model of the proton fast-ignition capsule implosion at the Omega Extended Performance facility, (3) completed diagnostic development of dualwavelength XUV emission imaging, (4) continued implementation of spatially resolved XUV spectroscopy, and (5) successfully adapted the Large-Scale Plasma code to model proton focusing, optimization of proton conversion efficiency, and energy deposition.

\section{Proposed Work for FY07}

We plan to (1) carry out three experimental campaigns on the Titan laser to explore electron transport in warm or hot plasmas, proton conversion efficiency from advanced hydrogen-rich targets, and optimization of proton focusing with shaped targets; (2) deliver a target design that can be fielded at the Omega Extended Performance facility with the optimum K-alpha fluorescence and thermal emission diagnostics to determine how much short-pulse particle energy was delivered to the target; (3) study electron transport within cone-wire surrogate targets on the Vulcan laser; and (4) submit our results to a highvisibility, peer-reviewed journal.

\section{Publications}

Li, C. K., et al., 2006. "Measuring E and B fields in laser produced plasmas through monoenergetic proton radiography." Phys. Rev. Lett. 97, 135003. UCRL-JRNL-220964.

Li, C. K., et al., 2006. "Monoenergetic proton backlighter for measuring $E$ and B fields and for radiographing implosions and HED plasmas." Rev. Sci. Instrum. 77, $10 \mathrm{E} 725$. UCRL-JRNL-221036.

Mackinnon, A. J., et al., 2006. "Proton radiography of a laser driven implosion." Phys. Rev. Lett. 97, 045001. UCRL-JRNL-210529.

Notely, M. M., et al., 2006. "Development of time resolved x-ray spectroscopy in high intensity laser plasma interactions." Rev. Sci. Instrum. 77, 10F322. UCRL-CONF-221180. 


\title{
Measuring Plasmon Density of States in Dense Matter
}

\author{
Otto L. Landen \\ 05-ERI-003
}

\begin{abstract}
This research conducts novel $x$-ray scattering experiments that demonstrate the measurement of optical properties of dense matter. Almost any type of dense material will be accessible by this novel technique, including hot, solid-density plasmas; compressed matter; and cold matter under high pressure such as occurs in inertial confinement fusion, laboratory astrophysics, material science, and high-energy-density experiments. The experiments will be conducted at multiple laser and synchrotron facilities, in collaboration with research teams at the University of California at Berkeley, Los Angeles, and San Diego.
\end{abstract}

The project is expected to accomplish five milestones: (1) measure plasmon states in cold dense matter, (2) demonstrate improved data quality and accuracy using the narrow-band scattering technique in the noncollective regime, (3) measure the plasmon spectra in isochorically heated plasmas and in shock-compressed matter, (4) test theories of dense matter with plasmon spectra and independent measurements of temperature and density using noncollective scattering, and (5) achieve complete determination of the plasmon density of states in cold matter and in dense plasmas. The results will be used to test theoretical models in regimes where no experimental data have been obtained previously.

\section{Mission Relevance}

Measuring the optical properties of dense matter will provide experimental data in high-energy-density regimes that are important for the stockpile stewardship mission. This project supports Lawrence Livermore's mission in breakthrough science, nurtures collaborations with the high-energy-density physics research community, and attracts talented scientists to the Laboratory.

\section{FY06 Accomplishments and Results}

As a major breakthrough in FY06, we achieved the first observation of plasmon resonances from isochorically heated, solid-density beryllium plasma at the Omega laser facility. Furthermore, we (1) demonstrated that improvements in overall detection efficiency enable x-ray scattering experiments to be conducted at smaller-scale laser facilities, (2) obtained scattering spectra from shock-heated lithium hydride at Lawrence Livermore's two-beam laser facility Janus, (3) performed the first K-alpha conversion efficiency measurements at Livermore's petawatt-class laser Titan, and (4) presented several invited talks at international conferences and university colloquia, and submitted our research results to peer-reviewed journals. 


\section{Proposed Work for FY07}

In FY07, new experiments at the Omega, Vulcan, FLASH, and Jupiter lasers have been scheduled to complete validating dense matter characterization with $\mathrm{x}$-ray scattering on plasmons. Experiments at the Omega laser using our successful experimental platform will yield novel experimental data on plasmon dispersion, and hence on the plasmon density of states. Furthermore, improvements in the signal-to-noise ratio on Vulcan and FLASH will enable observation of an up-shifted plasmon resonance, yielding a model-independent temperature measurement based on detailed balance, which relates the rate or probability of a reaction to its time-reversed process. The capability of the collective scattering technique to directly assess electron density will be further exploited in measuring plasmon resonances in shocked plastic at the Omega and Jupiter lasers.

\section{Publications}

Glenzer, S. H., 2006. Compton scattering in solid-density plasmas. Radiative Properties of Hot Dense Matter, Albufeira, Portugal, Sept. 11-15, 2006. UCRL-PRES-224297.

Glenzer, S. H., 2007. Dense plasma characterization with x-ray scattering. International Workshop Physics of High Energy Density in Matter, Hirschegg, Austria, Jan. 28Feb. 2, 2007. UCRL-PRES-218720.

Glenzer, S. H., 2006. Observation of plasmons in dense plasmas with x-ray scatter. 33rd Institute of Physics Ann. Conf. Plasma Physics, Crieff, Scotland, UK, Apr. 10-13, 2006. UCRL-PRES-221068.

Glenzer, S. H., 2006. Peak brightness experiments. UCRL-PRES-221072.

Glenzer, S. H., 2006. Thomson scattering experiments in warm dense matter. Physics of Non-ideal Plasmas, Darmstadt, Germany, Sept. 4-8, 2006. UCRL-PRES-224156.

Glenzer, S. H., 2005. Warm dense matter characterization with $x$-ray Compton scattering. UCRL-ABS-215219.

Glenzer, S. H., 2006. X-ray scattering measurements from solid density plasmas. 29th European Conf. Laser Interaction with Matter, Madrid, Spain, June 11-16, 2006. UCRL-PRES-221896.

Glenzer, S. H., 2006. X-ray scattering measurements on solid density plasmas. 33rd Institute of Physics Ann. Conf. Plasma Physics, Crieff, Scotland, UK, Apr. 10-13, 2006. UCRL-ABS-218545.

Gregori, G., et al., 2006. "Generalized x-ray scattering cross section from nonequilibrium solids and plasmas." Phys. Rev. E 74, 026402. UCRL-JRNL-219854.

Gregori, G., et al., 2006. "Limits on collective x-ray scattering imposed by coherence." Europhys. Lett. 74, 637. UCRL-JRNL-216107. 
Gregori, G., et al., 2006. "Measurement of carbon ionization balance in high-temperature plasma mixtures by temporally resolved x-ray scattering." J. Quant. Spectrosc. Radiat. Tran. 99, 225. UCRL-JRNL-208894.

Neumayer, P., et al., in press. "Solid-density plasma characterization with x-ray scattering on the 200-J Janus laser." Rev. Sci. Instrum. UCRL-CONF-221178.

Patel, P. K., et al., 2006. "Integrated laser-target interaction experiments on the RAL petawatt laser." Plasma Phys. Contr. Fusion 47, 833. UCRL-JRNL-225356.

Theobald, W., et al., 2006. "Hot surface ionic line emission and cold K-inner shell emission from petawatt-laser-irradiated Cu foil targets." Phys. Plasmas 13, 043102.

UCRL-JRNL-217895.

Urry, M. K., et al., 2006. "X-ray probe development for collective scattering measurements in dense plasmas." J. Quant. Spectrosc. Radiat. Tran. 99, 636. UCRL-JRNL-211559.

\title{
Understanding the Nuclear Magnetic Fields
}

\author{
Peter Beiersdorfer 05-LW-006
}

\begin{abstract}
There is reasonable doubt if recently developed models of magnetic field generation in nuclei are correct, because a spectral line predicted by these models could not be found in a laser-excitation experiment. Correct models are needed for interpretation of parity nonconservation (PNC) experiments, as well as for nuclear materials and energy research. We propose to conduct high-precision measurements of the hyperfine structure in praseodymium, thallium, and bismuth to provide data needed to develop correct nuclear models and to distinguish between current models for $n=2$ electron energy splittings in lithium-like ions. The experiment, carried out at the LLNL Super Electron Beam Ion Trap, utilizes passive emission spectroscopy with a high-resolution, soft-x-ray spectrometer.
\end{abstract}

Standard Model uncertainties stem largely from the uncertainties of various nuclear parameters. An improved knowledge of these parameters will be essential for further progress with PNC experiments. This project provides the necessary data to evaluate and distinguish among different models for generating magnetic fields in the nucleus, both near to and far from the "doubly magic" (proton- and neutron-closed shells) nucleus of lead-208. The results will guide improved descriptions of the nuclear-atomic interaction and help select those needed for accurate evaluation of PNC experiments. Our measurements will be the first to use antisymmetric wave functions to probe nuclear magnetic fields differently.

\section{Mission Relevance}

The proposed work, at the interface of atomic and nuclear physics, supports the Laboratory's stockpile stewardship mission and reinforces LLNL's mission in breakthrough 
science and technology. The project encourages collaborative efforts for the precision study of nuclear-atomic interactions and will attract talented scientists to the Laboratory.

\section{FY06 Accomplishments and Results}

In FY06, our instrumentation was successfully modified for wavelength measurements from 10 to $140 \AA$. We applied our new capability to measuring the 0.20 -eV splitting of praseodymium-141 ( $Z=59)$ by observing lines near $90 \AA$. This measurement of the hyperfine splitting far from the doubly magic nucleus of lead-208 ( $Z=82, N=126)$ was a very stringent test of nuclear field models, which must deal with a complicated open nuclear shell. We also finished our analysis of bismuth-209 $(Z=83)$ ions. Because bismuth-209 is the nucleus closest to the doubly magic lead-208 nucleus, these experiments have provided definitive insights into magnetic field generation. We were able to distinguish between predictions from different models, which allowed us to rule out models not in agreement with our data.

\section{Proposed Work for FY07}

In FY07, we will complete our project by measuring the hyperfine splitting effects of antisymmetric wave functions. The SuperEBIT electron beam ion trap will be used to measure $2 p$ excited-level hyperfine splitting in beryllium-like ions of bismuth. Because our observations in FY06 on lithium-like ions of bismuth revealed some of the hyperfine splittings of $2 p$ levels to be quite large, this experiment in FY07 will help specify the effects of nuclear-atomic interaction, which theory will have to match. The proposed measurements will be performed as a final step in our effort to provide the most accurate and complete data possible for understanding nuclear magnetic-field generation.

\section{Publications}

Beiersdorfer, P., in press. "Testing high- $Z$ QED with SuperEBIT: an estimate of the ${ }^{91+} U 1 \mathrm{~s}$ two-loop Lamb shift based on a measurement of the $2 \mathrm{~s}_{1 / 2}-2 \mathrm{p}_{1 / 2}$ transition in ${ }^{89+} \mathrm{U}$." Radiat. Phys. Chem. UCRL-JRNL-217517.

Trabert, E., 2006. Atomic spectroscopy of trapped, highly charged, heavy ions. 2006 Intl. Conf. Trapped Charged Particles and Fundamental Physics, Parksville, Canada, Sept. 3-8, 2006. UCRL-ABS-220789.

Trabert, E., and L. J. Curtis, 2006. "Isoelectronic trends of line strength data in the Li and Be isoelectronic sequences." Physica Scripta 74, C46. UCRL-JRNL-220013.

Trabert, E., et al., 2006. Experiment and theory in interplay on high-Z few-electron ion spectra from foil-excited ion beams and electron beam ion traps. 2006 Intl. Conf. Physics of Highly Charged Ions, Belfast, U.K., Aug. 28-Sept. 1, 2006. UCRL-ABS-219953. 


\title{
High-Pressure, Multimegabar Conductivity Experiments on Hydrogen: The Quest for Solid Metallic Hydrogen
}

\author{
Damon D. Jackson 05-LW-027
}

\begin{abstract}
Ultradense hydrogen has long been the subject of intense experimental and theoretical research because of, in part, the fascinating physics that arise from this seemingly simple system. Metallic hydrogen has been considered to be the prototypical system for the study of insulator-to-metal (I-M) transitions, and has been predicted to have a highpressure, high-temperature superconducting transition near $100 \mathrm{~K}$. We plan the first direct measurement of the expected I-M transition above $300 \mathrm{GPa}$ using designer diamond anvils, membrane diamond anvil cells, and the cryogenic loading of hydrogen. These three techniques were developed and are in routine use at Lawrence Livermore, and will be combined for the first time to investigate the possibility of solid metallic hydrogen.

This project leverages previous work at the Laboratory in determining the properties of hydrogen at high pressures, beginning with the discovery of fluid metallic hydrogen at high temperatures and pressures. Current efforts in the search for solid metallic hydrogen have focused on optical properties such as optical absorption, Raman spectroscopy, and $x$-ray diffraction. Designer diamond anvils will allow us to obtain direct measurements of the electrical resistivity of the various high-pressure phases of hydrogen. The discovery of solid metallic hydrogen would be a scientific breakthrough in not only high-pressure research, but also basic solid-state physics, in which hydrogen is commonly used as the simplest example of an I-M transition.
\end{abstract}

\section{Mission Relevance}

A detailed understanding of the properties of hydrogen at high pressure is important to stockpile stewardship and will also benefit work in planetary science and hydrogen energy storage in support of LLNL missions in breakthrough science and energy security.

\section{FY06 Accomplishments and Results}

In FY06, the final year of this project, we worked to overcome two critical experimental issues. The first was to improve upon the geometry of designer diamond technology to successfully reach multimegabar pressures. Toward this goal, we improved the lithography by reducing the probe width, which allows for a smaller diamond flat size, ultimately reducing it to $40 \mu \mathrm{m}$. This substantial improvement to designer diamonds will be used routinely for future multimegabar experiments. Our second experimental issue was to overcome the embrittlement of the diamond from hydrogen. This has been a major problem for research groups throughout the world, and we were not able to overcome this difficult hurdle. 


\title{
Novel High-Energy-Density Source
}

\author{
James H. Hammer 06-SI-001
}

\begin{abstract}
With this project, our objective is to develop a novel high-energy-density source of higher quality than achievable by other pulsed-power technology. The development of this novel source relies on a close coupling between multidimensional code simulations and experiments.

We expect to achieve a high-energy-density source that will be of broad utility to the Stockpile Stewardship Program and that will provide a capability complementary to future fusion-class lasers.
\end{abstract}

\section{Mission Relevance}

The work directly supports Stockpile Stewardship Program efforts at Lawrence Livermore by providing a new high-energy-density source for experiments, as well as by validating the codes used for high-energy-density modeling.

\section{FY06 Accomplishments and Results}

Significant advances in performance of the core concept were achieved through improved target designs in FY06 laboratory experiments. Early tests of a novel thermal x-ray source suggest much higher performance is achievable than with competing techniques, with potential application to a range of high-energy-density experiments. Complete characterization of the source and its performance limits awaits further experiments in FY07 and beyond. In addition, new calculational models were developed in FY06. In particular, existing sub-grid fluid models were extended to include magnetohydrodynamic physics, and implemented in a simulation code. Comparison with the growing experimental database shows that the new model significantly improves our basic understanding of the relevant physics.

\section{Proposed Work for FY07}

In FY07, the project will continue to extend source development experiments, characterize output, and build a basic understanding of the physics underpinning the concept. We will (1) make further improvements in predictive capability by detailed comparisons of calculational models with data, as well as develop innovative variations on the core concept; (2) improve the sub-grid model through the DAKOTA optimization tool, coupled to a large number of simulations and constrained by experimental data; (3) perform direct simulation of fine-scale physics in massively parallel simulations on Livermore's Advanced Simulation and Computing platforms; and (4) using our best models, evaluate the physical limits on high-energy-density states achievable with our new source. 


\title{
Publications
}

Hammer, J. H., 2005. Magnetohydrodynamic turbulence model. American Physical Society 47th Ann. Mtg. Division of Plasma Physics, Denver, CO, Oct. 24-28, 2005. UCRL-PRES-216076.

Hammer, J. H., O. A. Hurricane, and M. C. Herrmann, 2006. A novel high energy density source. UCRL-TR-219336.

\section{Active Detection and Imaging of Nuclear Materials with High-Brightness Gamma Rays}

\author{
Christopher P. Barty 06-SI-002
}

\begin{abstract}
This proposal leverages LLNL's world-leading capabilities in laser science, $x$-ray source development, accelerator physics, nuclear science, and detector technology to enable a new class of active interrogation techniques. The use of new linear accelerator(linac-) and laser-based gamma-ray sources for isotopic imaging provides an effective means of detecting concealed highly enriched uranium (HEU), including uranium-235. Isotopic selectivity is based on nuclear resonance fluorescence (NRF) from target nuclei. Specifically, these efforts include (1) measuring NRF transitions in uranium-235 and plutonium-239; (2) developing, modeling, and demonstrating linac/laser-based, highbrightness $x$-ray generation; (3) demonstrating NRF detection of uranium-238; and (4) developing modeling tools for optimizing NRF-based detection and imaging.

If successful, this project will create the world's highest brightness gamma-ray source and enable, for the first time, an effective detection modality for hidden HEU, as well as isotopic imaging. This detection capability could launch "nuclear photo-science" as a new field of study and result in numerous follow-on applications, and represents a solution to a longstanding challenge of significant importance to homeland security and nonproliferation.
\end{abstract}

\section{Mission Relevance}

The ability to detect and image HEU would support LLNL's national security mission, specifically homeland security and counterproliferation. Furthermore, the picosecond, high-spatial-resolution, tunable, mega-electronvolt source capability developed as part of this proposal would also impact a wide range of applications of importance to stockpile stewardship (in support of national security) and high-energy-density science and technology (in support of national and energy security).

\section{FY06 Accomplishments and Results}

In FY06, we (1) discovered a strong NRF transition in uranium-235 at $1733 \mathrm{keV}$, suitable for isotopic imaging of concealed HEU; (2) designed a symmetrized radio-frequency gun 
that suppresses parasitic modes and yields $0.6-\mathrm{mm}-\mathrm{mrad}$ emittance at $1 \mathrm{nC}$ and a $120-\mathrm{MV} / \mathrm{m}$ gradient in simulations; (3) developed a quadrupole linac e-beam transport system that preserves $0.7 \mathrm{~mm}$-mrad at the interaction point; (4) configured an existing laser to explore production of optimal ultraviolet pulses for the photocathode; (5) designed and began construction of the seed laser and new photocathode drive-laser systems; (6) developed a three-dimensional, nonlinear Compton code; and (7) began design of the interaction laser system, final focus, and interaction point.

\section{Proposed Work for FY07}

In FY07, we propose to (1) complete a 1-J, 355-nm, 10-ps drive laser, including diagnostics; (2) finalize the e-beam focus and interaction point construction, systems integration, and testing; (3) identify new applications for our Compton scattering light source for nuclear photoscience; (4) design and model NRF detection schemes for uranium-238 (NRF at $680 \mathrm{keV}$ ) and lithium (NRF at $477 \mathrm{keV}$ ) and perform inverse-density radiograph experiments; (5) design and model laser nonlinear trapping and recirculation and $\mathrm{x}$-ray active scanning; (6) characterize and optimize the $\mathrm{x}$ rays produced by the Compton scattering light source and benchmark our design codes; and (7) perform uranium K-edge (116 keV) and lithium NRF experiments.

\section{Publications}

Barty, C. P. J., et al., 2006. Nuclear photo-science and applications with Thomson-radiated extreme $x$-ray (T-REX) sources. LINAC 2006, Knoxville, TN, Aug. 2006.

UCRL-ABS-220558.

Barty, C. P. J., et al., 2006. Possibilities for nuclear photo-science with intense lasers. Intl. Conf. Ultrahigh Intensity Lasers, Cassis, France, Sept. 25-29, 2006. UCRL-ABS-222214.

Gibson, D. J., et al., 2005. Energetic $x$-ray production using Compton scattering sources. UCRL-ABS-217019.

Gibson, D. J., et al., 2005. Laser system for photoelectron and $x$-ray production in the PLEIADES Compton light source. Particle Accelerator Conf. 2005, Knoxille, TN, May 16-20, 2005. UCRL-PROC-211670.

Gibson, D. J., et al., 2006. High-brightness electron beams for high brightness $x$-ray and gamma-ray production. Advanced Accelerator Concepts 2006, Lake Geneva, WI, July 10-15, 2006. UCRL-POST-222332.

Gibson, D. J., et al., 2006. High-energy scaling of Compton scattering sources. APS Division of Plasma Physics Ann. Mtg. 2006, Philadelphia, PA, Oct. 20-Nov. 3, 2006. UCRL-POST-216040.

Gibson, D. J., et al., 2006. Numerical optimization of electron beams for high-brightness $x$ - and gamma-ray production. Advanced Accelerator Concepts 2006, Lake Geneva, WI, July 10-15, 2006. UCRL-PROC-223949.

Hartemann, F. V., et al., 2005. High-energy Compton scattering sources. Particle Accelerator Conf. 2005, Knoxville, TN, May 16-20, 2005. UCRL-POST-213309. 
Hartemann, F. V., et al., 2006. "Classical theory of Compton scattering: assessing the validity of the Dirac-Lorentz equation." Phys. Rev. E. 72, 026502. UCRL-JRNL-209955.

Hartemann, F. V., et al., 2006. "High-energy scaling of Compton scattering light sources."

Phys. Rev. Spec. Top. Accel. Beams 8, 100702. UCRL-MI-208147.

Hartemann, F. V., et al., 2006. Compton scattering light sources: high-energy scaling, nonlinear effects, and applications. Frontier Science Res. Conf., July 10-12, 2006, La Jolla, CA. UCRL-PRES-213140.

Pruet, J. A., et al., 2006. "Detecting clandestine materials with nuclear resonance fluorescence." J. App. Phys. 99, 123102. UCRL-TR-219071.

\title{
The Ultrafast Lattice Response of the Shocked Solid
}

Hector E. Lorenzana 06-SI-004

\begin{abstract}
Our goal is to perform the first studies to investigate real-time lattice level dynamics of shocked materials at characteristic temporal and spatial scales of the controlling physical phenomena. We have developed ultrafast $x$-ray diffraction and scattering diagnostic techniques that use the new generation of bright, ultrafast $x$-ray sources to obtain unambiguous measurements of lattice response. We will characterize, in situ, two phenomena: condensed matter phase transformations and incipient damage. We will couple our experimental results with state-of-the-art simulations which, for the first time, will match the length and time scales of the experiments. These results will lay the foundation for physics-based models of macroscopic material behavior under extreme conditions.
\end{abstract}

We expect to perform the first comprehensive real-time, in situ study of the lattice response under shock loading, a problem of great scientific importance. Properties of solids undergoing phase transitions, plastic deformation, or void growth during a shock are largely unknown, yet this information is crucial to predictive understanding of material response and failure. Success in this endeavor through laser and synchrotron-based experiments coupled with simulations will position LLNL at the forefront of a new field of high-energy-density materials science. The close coupling of experiment and theory will validate existing modeling at the atomistic, mesoscopic, and continuum scales, and spur the development of new models.

\section{Mission Relevance}

Success in stockpile stewardship, high-energy-density science, and other national security mission areas demand a fundamental understanding of dynamic materials behavior. The expertise developed in this project will further current and future research in support of stockpile stewardship at facilities such as the Joint Actinide Shock Physics Experimental Research (JASPER) gas gun at the Nevada Test Site, and basic science facilities such as the Linac Coherent Light Source at Stanford. This work will drive development of 
computational tools for predicting the performance, safety, and reliability of nuclear weapons, as well as for high-power laser research.

\title{
FY06 Accomplishments and Results
}

In FY06 we (1) directly observed a shock-driven phase transformation in titanium-the second-ever phase transition observed in a shocked metal by in situ $x$-ray diffraction; (2) designed an experimental setup to simultaneous conduct diffraction and velocimetry measurements, displaying unique correlations between atomistic-level phenomena and bulk response; (3) started microstructural studies of the solid-solid phase transformation in shocked iron-first-of-a-kind diffuse scattering measurements that are providing constraints on and insight into grain size and transition times during shock; and (4) developed a technique for using large supercomputers at the Laboratory to generate simulated $x$-ray diffraction spectra from large molecular dynamics (MD) simulations, allowing enhanced interpretation of the experimental data.

\section{Proposed Work for FY07}

In FY07, we propose to continue a multidisciplinary study of the in situ lattice-level dynamic response of shocked solids. By varying the shock-driven orientation of single crystals and shocking polycrystalline samples, we will explore the kinetics of atomic rearrangement pathways, seeking to observe novel metastable phases. Both MD simulations and experiments will be used to investigate two phenomena: the elastic-toplastic transition and the coexistence region of a solid-solid phase transformation. We will also begin studies of the solid-melt phenomena in bismuth, seeking to understand its kinetics and microstructure. Finally, we will perform in situ studies of damage at a synchrotron using small-angle $x$-ray scattering techniques.

\section{Publications}

Hawreliak, J. H., et al., in press. "An analysis of the x-ray diffraction signal for the alphato-epsilon transition in shock-compressed iron: simulation and experiment." Phys. Rev. B. UCRL-JRNL-220707.

\section{Laser-Driven Dynamic Hohlraums}

\section{Sharon G. Glendinning 06-ERD-017}

\begin{abstract}
With this project, we propose to use experiments and simulations to explore the laserdriven dynamic hohlraum (LDDH), a laser-driven analog to the dynamic hohlraum used at the Sandia $Z$ Machine, as an ignition target for future fusion-class lasers. The project will (1) investigate the neutron production history of capsules driven with an LDDH, (2) determine radiation production during an implosion, (3) use "polar" direct drive on the Omega laser, and (4) optimize the LDDH as a potential bright x-ray source and general
\end{abstract}


hohlraum driver. Experiments will be fielded on the Omega laser to test simulations of the LDDH addressing each of these points.

We expect to demonstrate the feasibility of the LDDH as a candidate for an ignition target, for $\mathrm{x}$-ray source development, and for diverse high-energy-density physics experiments in regimes not otherwise achievable. A noncryogenic ignition option could allow for more ignition experiments at lower cost than currently feasible, making possible more experiments on the effects and uses of ignition.

\section{Mission Relevance}

The LDDH, once demonstrated, could become a platform for a variety of high-energydensity physics experiments that support Lawrence Livermore's stockpile stewardship mission. Radiatively driven shocks are also of great interest in basic science, especially astrophysics, and thus this project supports the Laboratory's mission in breakthrough science.

\section{FY06 Accomplishments and Results}

In FY06 we performed three sets of experiments on the Omega laser, all dedicated to implosions, with inner capsules filled with deuterium. We measured neutron yield, fuel and shell areal density, and radiation production. Compared with simulations, we did not see the originally predicted scaling of the neutron yields. This is now understood, and both yields and areal densities are correctly predicted as a result of fall-line behavior of these implosions. Finally, we compared implosions with and without the radiative shock characteristic of the LDDH. The x-ray yields of both types of targets were measured, but difficulties fielding the comparison targets, which have since been resolved, resulted in no areal density measurements for those targets. We anticipate the measurements later in FY07.

\section{Proposed Work for FY07}

During FY07 we will concentrate on optimizing the LDDH as a bright $\mathrm{x}$-ray backlighting source. Simulations and experiments will concentrate on small capsules that may be fielded with few beams. We anticipate that such backlighters will generate considerable interest for applications in opacity and extended $\mathrm{x}$-ray absorption, fine-structure experiments. In addition, we will begin a study of the effect of nonuniform irradiation (polar direct drive) on shell symmetry (through x-ray imaging) and on neutron yield for comparison with simulations. The LDDH will be simulated with a polar-direct-drive geometry at a future fusion-class laser facility. Finally, we will complete the target comparison experiments begun in FY06.

\section{Publications}

Glendinning, S. G., and M. C. Herrmann, 2005. Laser driven dynamic hohlraums: implosions. 47th American Physical Society Division of Plasma Physics Mtg., Denver, CO, Oct. 26, 2005. UCRL-PRES-216302. 


\title{
High-Resolution Measurements of the Plasma Current Profile for ITER
}

\author{
Steven L. Allen 06-ERD-024
}

\begin{abstract}
The presently envisioned method of measuring the plasma current profile in advanced tokamaks is based on the motional Stark effect (MSE). When a high-energy neutral beam interacts with the plasma in a magnetic field, the MSE results in a polarized photon that can be used to measure the local plasma current and electric field. This project will study the possibility of making high-resolution measurements of the plasma current profile on burning plasma experiments, starting with the International Thermonuclear Experimental Reactor (ITER), for example, and leading to experiments with more advanced capabilities. We will model the burning plasma, simulate the atomics physics processes in the plasma, and trace the photons through the optical system in a plasma, neutron, and stray-light environment.
\end{abstract}

We will carry out a pre-conceptual study of an MSE diagnostic on several levels. On the modeling side, the plasma code CORSICA will be upgraded with synthetic diagnostics to calculate the actual MSE signals in burning plasma. As the measurement will be in a highneutron environment, we will develop the capability to optimize the optical design with the constraint of neutron shielding. Adequate shielding favors multiple mirrors, but accurate measurements favor a simple, no-mirror system. Collisional radiative modeling will be used to calculate signal-to-noise ratios and develop an in situ calibration. Polarizationsensitive optics may be degraded by plasma exposure, and we would start these measurements in existing devices.

\section{Mission Relevance}

This project will contribute to burning plasma fusion research, an important step in achieving fusion power for the country's long-term energy needs. The combined development of a new diagnostic tool and a new predictive code for ITER scenarios will advance plasma physics research, in support of LLNL and DOE's missions in fundamental science and energy security.

\section{FY06 Accomplishments and Results}

In FY06 we (1) developed a framework for iterative design tradeoffs between performance and neutron shielding (multiple mirrors for neutron shielding versus a simple linear system to preserve the polarization); (2) installed a prototype of an in situ monitor to measure the polarization performance of a mirror placed inside the DIII-D tokamak (and determined that a modification of the prototype is necessary for good measurements); (3) began characterization of polarization-sensitive optical components (metal mirrors) suitable for a burning plasma; and (4) started work on a code to study the effect of non-ideal polarimetry effects, noise, and stray light. 


\title{
Proposed Work for FY07
}

We will (1) use the CORSICA-MSE model to estimate signal-to-noise ratios for burning plasma operation; (2) compute optical performance (i.e., sensitivity and polarization blur); (3) begin developing a collisional radiative model of the MSE spectrum; (4) analyze the properties of mirrors exposed to plasma; (5) demonstrate a design tradeoff study for reducing the number of mirrors; and (6) initiate work on the measurement and characterization of polarization-preserving optical systems.

\section{Investigating New Regimes of Material Strength at Ultrahigh Strain Rates and Pressures}

Stephen M. Pollaine 06-ERD-027

\begin{abstract}
In this project, we will measure material strength of metals at high pressures ( $>1$ Mbar) and strain rates up to $10^{8} / \mathrm{s}$ by developing three Velocity Interferometer System for Any Reflector (VISAR) techniques: compression wave reverberations in thin samples, hysteresis between loading and unloading waves in thick samples, and VISAR measurements of Rayleigh-Taylor feedthrough from the front to the back surface of a metal foil undergoing acceleration. A new hohlraum-isentropic compression experiment (ICE) drive will serve as a platform for these techniques. This new technology will test various models of material strength, such as the Preston-Tonks-Wallace model, and provide parameters for them. This project will be exploring new regimes never before measured, where current models of strength differ dramatically from each other.
\end{abstract}

We expect to develop three new experimental techniques for measuring strength and a new hohlraum-ICE drive platform that will deliver larger, more uniform loading than is currently possible. In this project, at the forefront of science, we will take the first-ever time-resolved measurements of the strength of nanocrystalline materials at ultrahigh strain rates. Also, our work will contribute to understanding the effect of strength on the shock processing of interstellar dust grains, which affects the size distribution of the grains and, indirectly, the rate of star formation. Our results will be submitted for publication in peer-reviewed physics and materials science journals.

\section{Mission Relevance}

The three new VISAR techniques will make it possible to measure the strength of materials important for stockpile stewardship on existing facilities, while the development of the hohlraum-ICE drive is a critical advance for stockpile stewardship experiments on fusion-class lasers. Investigating new regimes of material strength at high strain rates and pressures also contributes to Lawrence Livermore's mission in breakthroughs in fundamental science. 


\title{
FY06 Accomplishments and Results
}

In FY06, we designed and performed experimental hohlraum shots at the Omega laser facility. We showed that our ICE hohlraums remain open past $80 \mathrm{~ns}$, and obtained the first measurement of preheat at low temperatures in solid-density metal. These hohlraums were so successful that the high-energy-density physics researchers have adopted the design for use in their experiments. We also designed and performed Janus experiments that demonstrated the wave forms needed for hysteresis measurements of strength. These represent the first demonstration of a "reverse reservoir," in which the transition from a more dense to a less dense material in the ablating and stagnating reservoir causes an abrupt drop in the pressure on the target Finally, we obtained reverberation data on Janus, and showed the need for a kinetics model, which is now being incorporated into our hydrocode.

\section{Proposed Work for FY07}

In FY07, we plan to (1) use the reverse reservoir technique to obtain usable data for the hysteresis measurement of strength; (2) develop a new hohlraum design that generates less preheat; (3) develop the reverberation strength technique on Janus and obtain data on the Omega laser; (4) develop the backside Rayleigh-Taylor technique, with a new diagnostic that can see large-angle wave forms; and (5) design experiments for gas guns and the ZR laser pinch facility at Sandia National Laboratory.

\section{The Properties of Confined Water and Fluid Flow at the Nanoscale}

Eric R. Schwegler 06-ERD-039

\begin{abstract}
The properties of confined water affect a wide range of scientific and technological areas of interest, including protein folding, cell membrane dynamics, materials properties, and nanofluidic devices. Using a series of ab initio molecular dynamics simulations to understand how hydrogen bonding and solvation are modified at the nanoscale, we propose to develop computational tools to study fluids in confined geometries and to apply these techniques to probe the structural and electronic properties of water confined between hydrophilic and hydrophobic substrates.

The primary results of this project will be threefold: (1) a first-principles-based computational framework for investigating and characterizing liquids that are confined at the nanoscale, (2) a better understanding of how the properties of water change upon nanoscale confinement by hydrophobic and hydrophilic surfaces, and ( 3 ) a suitable empirical model that can be used to describe the flow of water in confined media.
\end{abstract}

\section{Mission Relevance}

This project will contribute to a detailed understanding of how the structural, electronic, and dynamical properties of water are modified upon nanoscale confinement, which 
is relevant to the development of nanoscale-material sensor technologies for national security missions in nonproliferation and counterproliferation, as well as for homeland security missions in counterterrorism.

\section{FY06 Accomplishments and Results}

During FY06, we examined the properties of water confined by a variety of substrates, focusing on long-time-scale ( 20 ps) ab initio molecular dynamics simulations of confined water. The simulations were designed to address issues such as confinement length, dimensionality of the confining media, and interface effects (e.g., hydrophobic versus hydrophilic surfaces). In addition, we have developed a robust strategy for computing the $\mathrm{x}$-ray absorption spectra of materials within density functional theory, and benchmarked our approach on bulk liquid water and ice.

\section{Proposed Work for FY07}

In FY07, we propose to (1) perform a series of ab initio molecular dynamics simulations with hydrophobic and hydrophilic substrates to examine the effects of confinement on ion solvation properties, (2) evaluate the use of empirical potentials that explicitly account for polarization effects to reproduce the properties of confined water, and (3) determine the spectroscopic signatures of confined water, including a detailed examination of the $\mathrm{x}$-ray absorption spectra of water confined by representative hydrophobic and hydrophilic surfaces.

\section{Publications}

Allesch, M., E. Schwegler, and G. Galli, in press. "Structure of hydrophobic hydration of benzene and hexafluorobenzene from first principles." J. Phys. Chem. B.

UCRL-ABS-201465.

Lin, J. F., E. Schwegler, and C. S. Yoo, in press. "Phase diagram and physical properties of $\mathrm{H}_{2} \mathrm{O}$ at high pressures and temperatures: applications to planetary interiors." American Geophysical Union monograph. UCRL-JRNL-215773.

Prendergast, D., and G. Galli, 2006. "X-ray absorption spectra of water from first principles calculations." Phys. Rev. Lett. 96, 255003. UCRL-JRNL-221846.

\section{Spheromak Energy Transport Studies via Neutral Beam Injection}

David N. Hill

06-ERD-042

\section{Abstract}

Promising results from the Sustained Spheromak Physics Experiment (SSPX) at Livermore provide strong motivation for adding neutral beam injection (NBI) heating to study the physics of energy transport and pressure limits in spheromak fusion devices. The 
main objectives of this project are to (1) conduct simulation to determine the optimum geometry for NBI heating of spheromaks, (2) develop the physics basis of target plasmas suitable for NBI experiments, and (3) begin developing new plasma diagnostics in anticipation of NBI heating experiments.

Successful application of NBI heating to a spheromak plasma would be a significant step forward for the spheromak magnetic fusion concept. Past experience with other magnetic fusion experiments has shown NBI to be a powerful driver for significantly enhancing the quality of scientific output. We expect to (1) determine optimum conditions for application of NBI heating to spheromaks, (2) understand how NBI heating can be used to test pressure limits in the spheormak, and (3) apply state-of-the-art diagnostics to study of energy transport in a spheromak.

\section{Mission Relevance}

Plasma physics is at the core of Laboratory's national security mission. The proposed work would provide a test bed for computational simulations from the NIMROD threedimensional magnetohydrodynamics code. Research in magnetohydrodynamics theory and magnetic-field fluctuation theory also supports the Laboratory's mission in breakthroughs in fundamental science and technology.

\section{FY06 Accomplishments and Results}

Major accomplishments in FY06 include (1) developing a new algorithm for tracking fastion orbits in spheromaks and other similar magnetic confinement configurations, (2) using the CORSICA transport code to simulate neutral-beam heating in spheromaks, (3) using a new capacitor bank to produce long-pulse plasmas in SSPX, (4) testing a new soft x-ray diagnostic, (5) developing the design for a double-pulse Thomson scattering system, and (6) presenting these results at prominent national and international conferences.

\section{Proposed Work for FY07}

In FY07, we will (1) simulate beam heating with CORSICA using data from long-pulse plasmas produced by new spheromak experiments, (2) develop target plasmas suitable for application of NBI auxiliary heating and measurement of the time-history of electron temperature, (3) compute the pressure limit for predicted spheromak target-plasma conditions, and (4) complete the design for beam-based diagnostics for measuring ion temperature, plasma rotation, and plasma fluctuations.

\section{Publications}

Foster, R. L., et al., in press. Simulation of planned neutral beam heating experiments in SSPX. 48th Ann. Mtg. APS Division of Plasma Physics, Philadelphia, PA,

Oct. 30-Nov. 3, 2006. UCRL-ABS-223044.

Hill, D. N., et al., 2006. Confinement studies in high temperature spheromak plasmas. 21st IAEA Fusion Energy Conf., Chengdu, China, Oct. 16-22, 2006. UCRL-POST-225286. 
Hill, D. N., et al., 2006. Plans for auxiliary heating experiments on SSPX. Innovative Confinement Concepts 2006 Workshop, Austin, TX, Feb. 13-16, 2006. UCRL-POST-218975.

Ludington, A. R., et al., 2006. Soft x-ray measurements in SSPX. 48th Ann. Mtg. APS Division of Plasma Physics, Philadelphia, PA, Oct. 30-Nov. 3, 2006. UCRL-ABS-223078.

Pearlstein, L. D., et al., 2006. Calculations of neutral beam injection into SSPX. 33rd EPS Conf. Plasma Physics, Rome, Italy, June 19-23, 2006. UCRL-CONF-222188.

Wood, R. D., et al., in press. Spheromak buildup in SSPX using a modular capacitor bank. 48th Ann. Mtg. APS Division of Plasma Physics, Philadelphia, PA, Oct. 30-Nov. 3, 2006. UCRL-ABS-223041.

\title{
Heavy Quark-Jet Tomography of Compressed Nuclear Matter
}

\author{
Jennifer L. Klay 06-ERD-045
}

\begin{abstract}
We are investigating the compressed nuclear medium created in high-energy nuclear collisions at the Relativistic Heavy Ion Collider (RHIC) at Brookhaven National Laboratory and the Large Hadron Collider (LHC) at the European Organization for Nuclear Research (CERN) using heavy quark jets. The azimuthal correlations of electrons with associated charged hadrons can be used to identify jets containing heavy quarks, which lose energy as they traverse a quark-gluon plasma. We will use the Pioneering High-Energy Nuclear Interaction Experiment (PHENIX) experiment at RHIC to measure electron-hadron correlations in collisions among gold particles. The next frontier of heavy-ion physics is the LHC, scheduled to begin operations in FY08. We are preparing to use A Large Ion Collider Experiment (ALICE) at CERN to continue the study of heavy quark-jet tomography of the quark-gluon plasma at the higher energy available at LHC.
\end{abstract}

This research is of significant for heavy-ion physics and will contribute to our understanding of the dense matter formed at RHIC and LHC. Characterization of the mass dependence of jet quenching will help explain the underlying coupling of quarks and gluons in high-temperature nuclear matter and enable the quantitative evaluation of properties of the quark-gluon plasma. We expect to publish our results in peer-reviewed journals.

\section{Mission Relevance}

Jet tomography is a promising and active area of research in relativistic heavy-ion physics, which supports Lawrence Livermore's missions in stockpile stewardship, energy security, and breakthroughs in fundamental science and technology. Our scientific leadership in this area will attract top postdoctoral applicants to the Laboratory. Training postdoctoral researchers in relativistic heavy-ion physics has proven to be an effective means of cultivating talent and new detection concepts for the Laboratory's efforts in nonproliferation and homeland security. 


\title{
FY06 Accomplishments and Results
}

in FY06, we (1) developed the software tools to analyze electron-hadron correlations in heavy ion collisions, and began analyzing the signal for the existing 1.5 billion gold-collision events from PHENIX with this software while collecting baseline data from collisions of $p$-shell nuclei in the sixth RHIC run; (2) participated in the ALICE electromagnetic calorimeter test-beam studies; (3) developed a track-matching algorithm that enabled test-beam results to demonstrate that the detector meets the position and energy-resolution requirements of its design; and (4) successfully revived the offline software development team for the ALICE electromagnetic calorimeter, and completed necessary updates that enabled it to be used in a large, scheduled physics mock-data challenge.

\section{Proposed Work for FY07}

In FY07, we will (1) complete simulations needed for corrections and theoretical comparisons of the electron-hadron correlations at RHIC, and prepare a publication on results from the gold-gold collisions; (2) recruit top-quality postdoctoral applicants to work on PHENIX and ALICE and join our team to collect and analyze RHIC data from run seven; (3) continue to develop the ALICE electromagnetic calorimeter offline software; and (4) prepare to participate in the first LHC run of p-shell nucleus collisions.

\section{Publications}

Adare, A., et al., 2006. "Measurement of high-pT single electrons from heavy-flavor decays in $\mathrm{p}+\mathrm{p}$ collisions at sqrt(s) $=200$ GeV." Phys. Rev. Lett. 97, 252002. UCRL-JRNL-228383.

Klay, J. L., 2006. Jet measurements in the ALICE experiment at the LHC. IX Intl. Conf. Nucleus-Nucleus Collisions, Rio de Janeiro, Brazil, Aug. 28-Sept. 1, 2006.

UCRL-PROC-225837.

\section{Foam-Walled Hohlraums for Increased X-Ray Conversion Efficiency}

Peter E. Young

06-ERD-053

\begin{abstract}
Recent analytical and computational analysis of the radiation heat front in the high-atomicnumber wall of laser-irradiated hohlraum targets suggests that an optimum wall density exists for maximum radiation energy. The hypothesis of this project is that reducing wall density should result in two beneficial effects. First, it decreases the hydrodynamic energy of the wall upon absorption of the laser light, which thus increases the energy that goes into radiation. Second, it decreases the amount of plasma filling the hohlraum, which interferes with laser light entry into the hohlraum at later pulse times. The predicted increase in x-ray flux ( $20 \%)$ would provide an increased margin of energy in experiments using large laser facilities. This project plans to demonstrate, on the Omega laser, the
\end{abstract}


improvement in x-ray conversion efficiency and reduction in hohlraum filling using foamwalled, high-atomic-number hohlraums of different densities.

Successful demonstration of increased $x$-ray conversion efficiency and reduced hohlraum filling using foam-walled hohlraums will have an important impact on target designs for both inertial confinement fusion (ICF) and high-energy-density (HED) experiments planned for large laser facilities. Reduction of the required laser energy for a given $\mathrm{x}$-ray flux will increase the energy margin for such experiments and reduce the operating costs of those facilities by reducing damage to final optics. Once the physics of foam-walled hohlraums has been demonstrated, they will likely become a standard experimental platform for large lasers.

\section{Mission Relevance}

Target designs that increase the $x$-ray conversion and decrease the amount of hohlraum filling from ablated plasma are very important for the ICF and HED experiments planned for large laser facilities, which support Lawrence Livermore's national and energy security missions.

\section{FY06 Accomplishments and Results}

Using the Omega laser and foams made of tantalum oxide with densities of $200 \mathrm{mg} / \mathrm{cm}^{3}$ and $4 \mathrm{~g} / \mathrm{cm}^{3}$, we conducted initial experimental campaigns to test the dependence of $x$-ray flux on initial wall density. The observed flux dependence is quantitatively in agreement with analytical predictions. In addition, we addressed issues in hydrodynamic simulations (e.g., finer zoning in the walls) that have improved agreement with experiments. Gold foams of $500 \mathrm{mg} / \mathrm{cm}^{3}$ density have been cast and machined into hohlraums of the correct dimensions for comparison to those of solid gold at the Omega laser facility.

\section{Proposed Work for FY07}

In FY07, we plan to complete the comparison of low-density gold foam hohlraums to solid-gold hohlraums. We also will begin testing the dependence of the amount of ablated plasma on the initial wall density using Thomson scattering at the Omega laser facility.

\section{Mitigation of Electromagnetic Pulse Effects from Short-Pulse Lasers and Fusion Neutrons}

David C. Eder 06-ERD-055

\section{Abstract}

The main source of damaging electromagnetic pulses (EMP) at laser facilities is believed to be the small fraction of electrons that escape the target. We are exploring this theory at the new Titan laser facility, where we will measure the number and spatial distribution 
of escaping electrons as well as the resulting transient currents and EMP. The electron properties will be used in three-dimensional electromagnetic simulations of the Titan chamber using the code EMSolve. System-generated EMP (SGEMP) will be studied using a variety of codes. The EMP simulations will provide a quantitative understanding of the relationship between escaping electrons and EMP, and we hope to develop ways to reduce EMP by controlling the escaping electrons and structures that the electrons strike.

This project will provide the first quantitative understanding of EMP processes, including SGEMP in high-power laser facilities. The data obtained on the dependence of the number of escaping electrons and spatial distribution on laser and target conditions will greatly benefit research by validating simulation tools used to predict these quantities. Success of this project will result in a truly predictive simulation capability that can be applied to existing and future lasers facilities to mitigate EMP and greatly reduce the occurrence of diagnostic damage and data loss. Demonstration of EMP reduction will stimulate additional research in EMP mitigation, and detailed knowledge of the EMP from simulations also can be used to mitigate effects of EMP by helping develop better-designed conventional shielding.

\section{Mission Relevance}

The work supports stockpile stewardship in two ways. First, knowledge of EMP and system response is critical for reliable operation of facilities used to validate ignition simulations and simulate weapons effects. Second, possible future underground experiments require knowledge of SGEMP on components that did not exist during earlier testing. This work on EMP mitigation techniques also will apply to short-pulse lasers for fast ignition, in support of Lawrence Livermore's energy security mission.

\section{FY06 Accomplishments and Results}

In FY06, we (1) installed a significant instrumentation and electrical infrastructure, including a Faraday enclosure cableway system, in the Titan chamber area to allow accurate measurements of EMP inside and outside the chamber; (2) measured properties of the escaping electrons using image plates; (3) studied a range of target and laser conditions; (4) deployed an array of thermoluminescence detectors to measure the spatial dependence of the $x$ rays and gamma rays; (5) simulated the eigenmodes of the Titan chamber and started transient simulations of an electron bunch leaving a target and striking the chamber wall; (6) made frequency-response measurements with a 2-GHz probe and identified ways to reduce EMP background; and (7) using the probe signal, obtained an electric field value of about $50 \mathrm{kV} / \mathrm{m}$.

\section{Proposed Work for FY07}

In FY07, we will (1) explore EMP reduction by using different grounding choices for an electron beam dump; (2) study the effect on EMP of adding equipment into the chamber; (3) improve the model of the electron beam in EMSolve, given beam dump data;

(4) incorporate the electromagnetically transparent chamber windows into the simulation to compute how fast the modes die away from radiation, and determine how much energy enters the room; (5) compare computed and measured data on the time rate of 
change of the magnetic field inside and outside the target chamber; and (6) evaluate the relative importance of system-generated EMP by fielding cable configurations on Titan and conducting simulations.

\title{
Publications
}

Eder, D. C., et al., 2006. "Impact of neutron and gamma radiation on the design of NIF diagnostics and target-bay systems." J. Phys. IV Proc. 133, 907. UCRL-CONF-215039.

\section{Laser Beam Propagation in High-Temperature Plasmas}

Dustin H. Froula

06-ERD-056

\begin{abstract}
The primary goal of this research is to develop understanding of laser beam propagation through high-temperature plasmas. This research is of interest to numerous Laboratory programs, including material strength, radiation transport, and hydrodynamics. Experiments and numerical modeling will focus on blue (351-nm) high-intensity laser beams interacting with the high-temperature plasmas that will occur in high-energydensity (HED) physics experiments such as those at fusion-class lasers. Determining the limits of efficient laser beam propagation will strongly influence the design of a wide variety of experiments that scale strongly with laser intensity, and will allow us to access higher regimes of pressure, temperature, and strain rate in matter.

In this work, we will resolve important scientific questions relating to laser-plasma interactions in high-temperature plasmas, which are important for HED science applications. We will determine density, temperature, and laser beam intensity limits, which will serve as input parameters for the design of critical stockpile stewardship-related experiments. Limits due to plasma inhomogeneities by hydrodynamic plasma motion and magnetic field pressure, or by Landau damping due to high plasma temperature, will be directly measured with Thomson scattering. These data will provide a critical test for predicting the target physics design space using laser-plasma interaction calculations coupled with radiation-hydrodynamic modeling.
\end{abstract}

\section{Mission Relevance}

Five key areas within the Stockpile Stewardship Program would significantly benefit from the ability to predict the design space limits for laser-driven HED experiments:

(1) fusion ignition, (2) material dynamics, (3) experiments using special nuclear materials, (4) nuclear weapons effects testing, and (5) hydrodynamics. This project will also enable cutting-edge research in other LLNL mission areas such as inertial confinement fusion, in support of the energy security mission. 


\section{FY06 Accomplishments and Results}

In FY06, we implemented a new high-temperature laser-plasma interaction platform and made spatial measurements indicating an electron temperature (a critical parameter in laser beam propagation) of $3.5 \mathrm{keV}$. Experiments at these high temperatures demonstrated the limits of propagation for 351-nm laser beams to be $3 \mathrm{keV}$ for intensities less than $2 \times 10^{15} \mathrm{~W} / \mathrm{cm}^{2}$. Furthermore, the laser beam was shown to propagate without filamentation at these intensities. Initial experiments to test the density limits for propagation indicated a backscatter threshold of $10^{21} \mathrm{~cm}^{3}$.

\section{Proposed Work for FY07}

In FY07, we will experimentally measure the absolute transmission of a 351-nm beam propagating through hohlraum plasmas as a function of density by varying the initial gas density and increasing the propagation scale length to $4 \mathrm{~mm}$ while maintaining the 3-keV electron temperature. The occurrence of significant scattering losses or beam filamentation will thus be determined and compared to modeling using laser-plasma interaction codes such as pF3D. This work will strongly influence the design of a wide variety of experiments that scale strongly with laser intensity or electron temperature, and will allow us to access higher regimes of pressure, radiation temperature, and strain rate in future high-energydensity laser experiments.

\section{Publications}

Froula, D. H., et al., 2006. Thomson-scattering techniques in laser produced plasmas. 16th Topical Conf. High-Temperature Plasma Diagnostics, Williamsburg, VA. May 7-11, 2006. UCRL-PRES-221154.

Froula, D. H., et al., 2006. "0.351 nm transmitted beam diagnostic at the Omega Laser Facility." Rev. Sci. Instrum. 77, 10E507. UCRL-JRNL-221120.

Froula, D. H., et al., 2006. $351 \mathrm{~nm}$ transmitted beam diagnostic at the Omega Laser Facility. 16th Topical Conf. High-Temperature Plasma Diagnostics, Williamsburg, VA. May 7-11, 2006. UCRL-POST-221122.

Froula, D. H., et al., 2006. "Thomson-scattering measurements of high-electrontemperature hohlraum plasmas for laser-plasma interaction studies." Phys. Plasmas 13, 052704. UCRL-JRNL-219323.

Froula, D. H., et al., 2006. "Thomson-scattering techniques to diagnose local electron and ion temperatures, density, and plasma wave amplitudes in laser-produced plasmas." Rev. Sci. Instrum. 77, 10E522. UCRL-CONF-221161.

Froula, D. H., et al., 2006. 351-nm beam propagation in high temperature long-scale length plasmas. 36th Anomalous Absorption Conf., Jackson Hole, WY, June 4-9, 2006. UCRL-PRES-221688.

Froula, D. H., et al., 2006. Thomson-scattering techniques in laser produced plasmas. 16th Topical Conf. High-Temperature Plasma Diagnostics, Williamsburg, VA. May 7-11, 2006. UCRL-PRES-221154. 
Froula, D. H., et al., in press. "Ideal laser beam propagation through high-temperature ignition hohlraum plasmas." Phys. Rev. Lett. UCRL-JRNL-225079.

Ross, J. S., et al., 2006. "Implementation of imaging Thomson scattering on the Omega Laser." Rev. Sci. Instrum. 77, 10E520. UCRL-JRNL-221159.

Ross, J. S., et al., 2006. Implementation of imaging Thomson scattering on the Omega laser. 16th Topical Conf. High-Temperature Plasma Diagnostics, Williamsburg, VA, May 7-11, 2006. UCRL-ABS-219157.

\title{
Nuclear Physics the Monte Carlo Way
}

\author{
William E. Ormand 06-LW-013
}

\begin{abstract}
This research effort will investigate the application of Monte Carlo methods to the nuclear many-body problem. In this project, we will examine improvements and applications of the Auxiliary-Field Monte Carlo (AFMC) method, which can provide exact results for systems with extraordinarily large dimensions (e.g., $10^{20}$ and beyond), where brute-force matrix diagonalization methods are hopelessly overmatched. In the past, applications were limited because of the notorious "sign problem," which recently has been mitigated considerably and might be resolved more generally with this project.

With successful implementation of these Monte Carlo methods, the range of topics in nuclear theory that we can explore is far reaching. We will be able to (1) perform the first truly detailed studies of intermediate-mass nuclei at the limits of stability with realistic interactions; (2) compute weak transition rates important for theories of supernova collapse; (3) compute level densities, electro-weak rates, and binding energies important for nucleosynthesis in the r-process; and (4) study evolution of the giant-dipole resonance in nuclei at high excitation energy. Our work will provide a tool for future theoretical studies of nuclear structure. Results will be published in physics journals, and we hope to provide data that is important to the astrophysics community.
\end{abstract}

\section{Mission Relevance}

This project supports Lawrence Livermore's stockpile stewardship and homeland security missions by providing scientific basis and a new tool for theoretical studies in nuclear structure, which impacts the nuclear data needs of these missions. The project utilizes high-performance computing to address important problems in nuclear theory, thus supporting the Laboratory's mission in breakthroughs in fundamental science, and also will provide critical underpinning for the Rare-Isotope Accelerator, a proposed DOE nuclear physics facility. 


\title{
FY06 Accomplishments and Results
}

In FY06, we (1) examined, in collaboration with researchers at University of California at Los Angeles, implementations of the shifted-contour method to test its applicability; (2) implemented a Gaussian sampling method; (3) applied the AFMC method to test cases in which a direct comparison with diagonalization can be made; and (4) hired a postdoctoral employee to install computer codes on Laboratory supercomputers. The Gaussian sampling method exhibits better performance than previous sampling based on Metropolis. We also collaboratively performed parity-projected calculations for iron-56 to iron-68 nuclei with a pairing-plus-quadrupole interaction.

\section{Proposed Work for FY07}

Based on our successful FY06 tests and first applications, in FY07 we will (1) widen applications to nuclei in the 40 to 80 mass region, in which direct diagonalization is not possible; (2) compute and tabulate ground-state binding energies; and (3) compute level densities for astrophysical applications. The results will be compared with those of empirical models. To perform applications for heavier nuclei with a mass of 100 or more in FY08, we will need to examine some numerical stability issues. Numerical techniques, such as singular-value decomposition, will be implemented and tested for efficiency.

\section{Observation of Coherent Terahertz Frequency Emission from Shocked Polarizable Materials}

Evan J. Reed

06-LW-063

\begin{abstract}
We recently predicted that coherent electromagnetic radiation $(1-100 \mathrm{THz})$ can be generated in crystalline materials when subjected to a shock wave. This phenomenon represents a fundamentally new form of coherent optical radiation. The predicted coherent emission contains information regarding shock speed, lattice and atomic structure of the crystal, and shock-front rise time, suggesting this effect may represent a new experimental probe. In this project, we propose to perform the first-ever experimental measurements of terahertz emission from shocked materials and determine the ultrafast dynamical processes responsible for this emission. The experiments will utilize ultrafast laser-driven shock waves in polarizable materials, coupled with a theoretical effort to provide guidance and interpretation.
\end{abstract}

We expect to make the first observation of the emission of terahertz radiation from a shock. We will determine whether emission of coherent photons can be observed with existing technology, and extract the information contained in this emission regarding shock-front rise time, picosecond timescale shock-speed history, and the crystal lattice constant. This work could result in the observation of a fundamentally new source of 
coherent optical radiation, adding to an extremely short list of existing sources, which include the laser. We will assess the effect as a practical source of radiation and assess the utility of terahertz emission as a fundamentally new general probe into dynamical processes in shock waves. Our results will be submitted for publication in peer-reviewed journals.

\section{Mission Relevance}

New tools for understanding shock wave properties and materials phenomena are central to mission needs in stockpile stewardship. This work will provide fundamentally new insight into shock wave properties and phenomena in materials. It also provides a new source of terahertz radiation with unique properties, which can be used for applications in defending against chemical and biological threats, in support of the Laboratory's missions in national and homeland security.

\section{FY06 Accomplishments and Results}

In FY06, we met all our milestones, and published the first paper regarding theoretical potential coherent emission from shocked crystals. Our initial work was reported in highvisibility scientific publications such as Nature and Physics Today. In addition, we designed and fabricated thin films of the gallium nitride/aluminum nitride, piezoelectric crystals to be used in laser-driven shock experiments, and have made initial observations of terahertz frequency radiation emitted from these materials during generation of low-amplitude shocks and acoustic waves.

\section{Proposed Work for FY07}

In FY07, we will determine whether emission of coherent photons from the shock can be observed, and extract emission information regarding shock-front rise time, picosecond timescale shock-speed history, and the crystal lattice constant. Such an observation will ultimately yield a high-visibility paper reporting a fundamentally new source of coherent radiation. In the absence of such an observation we will determine, for the first time, the nature and origin of any other observed terahertz signals, possibly including dielectric breakdown and phase transformations.

\section{Publications}

Reed, E. J., et al., 2006. "Coherent photons from shock waves in crystals." Phys. Rev. Lett. 96, 043903. UCRL-JRNL-210215. 


\title{
Feasibility Study for Large, Water-Based Neutron and Neutrino Detectors
}

\author{
Robert C. Svoboda $\quad$ 06-FS-011
}

\begin{abstract}
We will construct a small, gadolinium-chloride-doped water Cherenkov detector to evaluate its use in large neutron and neutrino detectors. Currently, most detectors are made from organic liquids that are typically flammable, toxic, and expensive. Water Cherenkov technology solves these problems, but the energy of the capture gamma from hydrogen is too low to detect $(2.2 \mathrm{MeV})$. Capture on gadolinium gives an $8-\mathrm{MeV}$ gamma cascade, which can be seen by a water Cherenkov detector, but there are concerns that gadolinium salt might reduce water transparency in the ultraviolet, and accelerate corrosion of detector components.

We need to show that gadolinium does not significantly reduce water transparency in the ultraviolet from the typical $80 \mathrm{~m}$ achieved in current detectors. In addition, we must show sensitive detector components like weld spots and photomultiplier assemblies are not seriously affected by the presence of gadolinium salt if the water is de-oxygenated and sterilized. If successful, this will lead to a proposal to construct a small, water-based neutrino and neutron detector as the next phase. Both the Super-Kamiokande and Sudbury Neutrino observatories have expressed interest in using gadolinium to extend their scientific reach via efficient neutron detection. There also are plans for megaton-scale neutrino detectors in the U.S., Europe, and Japan that would use this technology, if available.
\end{abstract}

\section{Mission Relevance}

For basic science research, the proposed detector has direct and immediate application to projects that seek to understand the nature of neutrinos, a class of fundamental particles about which we know very little. The proposed detector represents a possible substitute for scintillator detectors now under study at Lawrence Livermore, in support of the Laboratory's mission in national and homeland security. This project also promotes collaboration with the University of California at Irvine, and supports LLNL's mission in discovery-class science. If successful, the project can enable economical, long-range detection of nuclear reactors for treaty verification applications.

\section{FY06 Accomplishments and Results}

In FY06, we built a 20-m-long attenuation arm and have instrumented it with a dye laser and appropriate readout electronics. The custom materials test tank was designed, completed, and loaded with photomultiplier assemblies for testing. In addition, we constructed a pure water system than will be able to serve both the materials tank and the transmission arm. Despite delays in getting the water system hut built and the optics in place, we initiated "first light" on October 26 . We are now essentially ready to make measurements of the gadolinium transparency as a function of concentration, and perform materials testing. 


\title{
Feasibility of Gratings for Increasing Solid-State Laser Gain and Efficiency
}

\author{
Alvin C. Erlandson 06-FS-014
}

\begin{abstract}
The objective of this study is to demonstrate the feasibility of increasing the efficiency and reducing the costs of solid-state lasers by using diffraction gratings on laser slabs. We expect diffraction gratings to increase efficiencies for coupling deleterious amplified spontaneous emission (ASE) out of laser slabs and for coupling pump light into laser slabs. If successfully developed, grating-enhanced laser slabs would represent a significant advance in solid-state laser technology that could benefit stockpile stewardship and fusionenergy research. We plan to design and fabricate a slab grating with the desired optical properties using existing grating capabilities at LLNL, and to measure slab gain in an existing laser laboratory.
\end{abstract}

If we succeed, we will contribute significantly towards solving a problem that has faced designers of large solid-state lasers for decades: gain clamping in laser slabs by internal ASE. We will also contribute to solving a more recent problem: how to more efficiently couple diode pump light into laser slabs. In the course of this work, we will (1) develop capabilities for modeling ASE and pump-light absorption in slabs that have gratings, (2) quantitatively calculate the improvements expected from various grating designs, and (3) demonstrate that gratings with the desired optical properties can be manufactured.

\section{Mission Relevance}

This research will improve performance and reduce costs for high-energy solid-state lasers of the types used in the Stockpile Stewardship Program. The gratings developed will have possible application to titanium-sapphire lasers, which are used for experiments in highenergy-density physics relevant to stockpile stewardship, laboratory astrophysics, and fast ignition. In addition, efficiency improvements could be pivotal for the feasibility of using solid-state lasers as drivers in inertial fusion energy power plants.

\section{FY06 Accomplishments and Results}

During FY06, we (1) developed a ray-trace model that calculates ASE decay rates for stored energy in grating-enhanced laser slabs; (2) developed grating designs for suppressing ASE in titanium-doped sapphire slabs and in neodymium-doped yttriumaluminum-garnet slabs, (3) fabricated a prototype grating on a 10-mm-thick, $100-\mathrm{mm}$ diameter titanium-doped laser slab, and (4) submitted a patent application for gratingenhanced, solid-state laser amplifiers. The best predicted performance for titanium-doped sapphire slabs was achieved with grooves with $2500 / \mathrm{mm}$ spacing etched into an antireflective coating consisting of a 170-nm-thick layer of tantala and a 120-nm-thick overcoat of silica. 



\section{Laboratory Directed Research and Development}

Lawrence Livermore National Laboratory

P.O. Box 808 , L-003

Livermore, California 94551

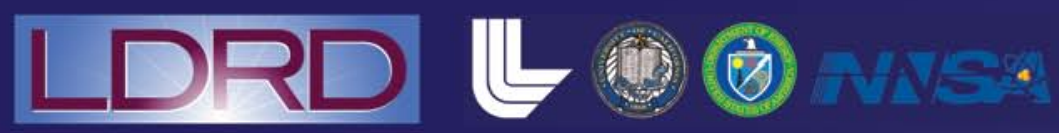

